SOME OBSERVATIONS CONCERNING THE USE OF REALISTIC FORCES IN

A MICROSCOPIC DESCRIPTION OF THE INELASTIC SCATTERING

OF NUCLEONS FROM NUCLEI AT MEDIUM ENERGIES

By

Fred I. Petrovich

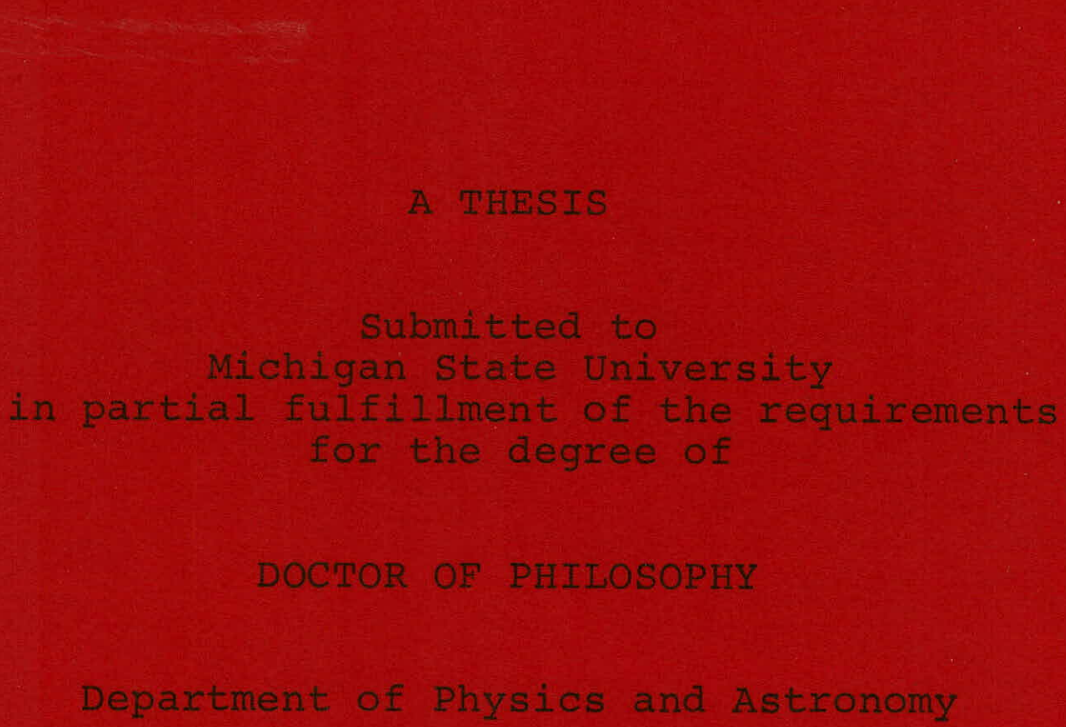




\section{DISCLAIMER}

This report was prepared as an account of work sponsored by an agency of the United States Government. Neither the United States Government nor any agency Thereof, nor any of their employees, makes any warranty, express or implied, or assumes any legal liability or responsibility for the accuracy, completeness, or usefulness of any information, apparatus, product, or process disclosed, or represents that its use would not infringe privately owned rights. Reference herein to any specific commercial product, process, or service by trade name, trademark, manufacturer, or otherwise does not necessarily constitute or imply its endorsement, recommendation, or favoring by the United States Government or any agency thereof. The views and opinions of authors expressed herein do not necessarily state or reflect those of the United States Government or any agency thereof. 


\section{DISCLAIMER}

Portions of this document may be illegible in electronic image products. Images are produced from the best available original document. 


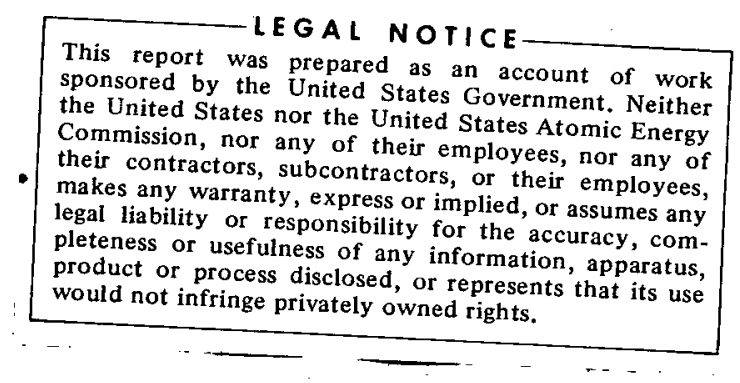

ABSTRACT

SOME OBSERVATIONS CONCERNING THE USE OF REALISTIC FORCES IN A MICROSCOPIC DESCRIPTION OF THE INELASTIC SCATTERING OF NUCLEONS FROM NUCLEI AT MEDIUM ENERGIES

By

Fred L. Petrovich

The problem of describing, in a microscopic picture, the process of inelastic nucleon-nucleus scattering at incident energies in the 15-70 MeV range is of current interest. of primary interest are the properties of the projectile-target interaction. In this work several models for this interaction are investigated by direct calculation. All of the interaction models considered are consistent with some portion of the data concerning the free two-nucleon force; hence, the term "realistic forces" which appears in the title of this paper. To be specific, it is assumed that the projectile-target interaction is given by (1) a pseudopotential derived from the impulse approximation, (2) the long range part of the Kallio-Kolltveit potential (K-K force) which is known to be a good approximation to the central part of the shell model reaction matrix, and (3). a Yukawa force derived from effective range theory. 
Fred L. Petrovich

This study is restricted in that the local distorted wave approximation (D.W.A.) is used throughout and no consideration is given to components of the interaction with complicated spin dependence such as the tensor and $\bar{l} \cdot \bar{s}$ parts. Approximations are made to treat the exchange component of the D.W.A. transition amplitude which is non-local. This component appears because of the required antisymmetrization of the projectile-target wave function and it has been neglected in most recent work on this problem. These approximations are discussed and some comparisons with exact calculations are presented.

Application is made to $\left(p, p^{\prime}\right)$ transitions in closed and pseudo-closed shell nuclei. Random phase approximation (R.P.A.) state vectors are used to describe the states of the target nuclei. Studies of the (e,e') reaction and the $\left(\mathrm{p}, \mathrm{p}^{\prime}\right)$ reaction (at incident energies in excess of 100 MeV) have shown that these vectors give a good description of the transitions considered; therefore, these calculations provide a test for the proposed interaction models. The results obtained with all three interaction models are shown to be in reasonable agreement with experiment, although the Yukawa effective range force appears to be somewhat poorer than the other two at incident energies below $30 \mathrm{MeV}$. The inclusion of exchange plays an essential part in giving this agreement. In most instances deficiencies in the shapes of the theoretical angular distributions are noted. 
Fred I. Petrovich

Further application is made to transition involving low lying states in nuclei which possess one or two nucleons outside of a closed shell. The purpose is to study core polarization effects which are known to be important in these transitions. The effects are estimated in calculations which use either a microscopic model or the macroscopic vibrational model to describe the core. Emphasis is on the completely microscopic calculations which assume that the core can be described by a zero order shell model Hamiltonian and that only the effect of simple particle-hole excitations of this core with energies up to roughly $2 \pi \omega$ need by considered. The coupling between the valence nucleons and the core is treated by first order perturbation theory and the $\mathrm{K}-\mathrm{K}$ force is taken to be the coupling interaction. This model is essentially the same as the one used recently by Kuo and Brown in work on the spectra of nuclei of this type. Contributions to $\left(p, p^{-}\right)$cross sections due to core polarization are large. The relation between the effect of core polarization on the spectrum and in inelastic proton-nucleus scattering is examined. The microscopic model doesn't do too badly on the $\left(p, p^{-}\right)$cross sections, 1.e. mass polarization effects. The experimental data is underestimated somewhat. However, effective charges for corresponding $\gamma$-transitions, i.e. charge polarization effects, are badly underestimated. One case is found where this model does badly, on the mass polarization. This is explained by explicltly taking into account the effect of a highly collective state in the core nucleus. 
Fred L. Petrovich

From this study it is concluded that a reasonable description of this class of reactions is obtained using "realistic forces" provided the treatment includes the effects of (1) antisymmetrization and (2) long range correlations in the target nuclei, in particular, core correlations (R.P.A.) and core polarization. 


\title{
SOME OBSERVATIONS CONCERNING THE USE OF REALISTIC FORCES IN A MICROSCOPIC DESCRIPTION OF THE INELASTIC SCATTERING OF NUCLEONS FROM NUCLEI AT MEDIUM ENERGIES
} By

Fred I. Petrovich

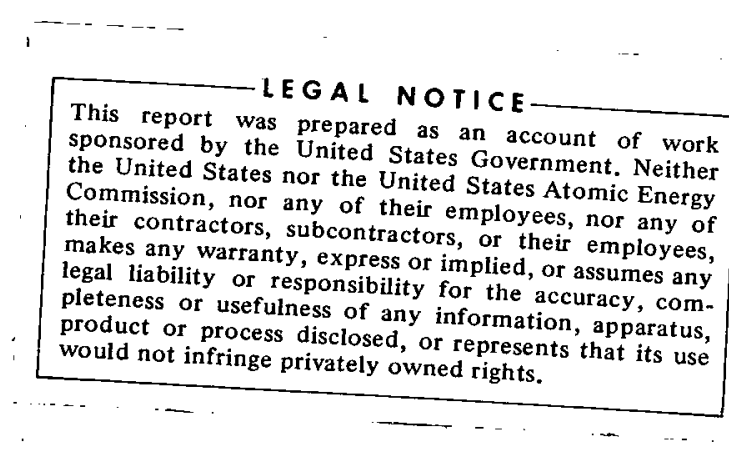

A PIESIS

\author{
Submitted to \\ Michigan State university \\ in partial fulfillment of the requirements \\ for the degree of \\ DOCTOR OF PHILOSOPHY \\ Department of Prysics and Astronomy
}

1970 


\section{ACKNOWLEDGEMENT}

I would like to thank Professor Hugh McManus for suggesting this problem and for his constant guidance and support during the time that this work was performed.

Thanks is also due to Dr. G. R. Satchler for assistance in the initial stages of this work, to Mr. J. Atkinson and Dr. V. A. Madsen for assistance with a particular phase of the work, and to the many people at the M.S.U. Cyclotron Laboratory whose interest in this work provided a constant source of encouragement. Mrs. Julie Perkins is also thanked for typing the manuscript.

Lastly, I would like to thank my wife, Paula, and my sons, Michael and Joseph, without whose patience and understanding this work would not have been possible. 
TABLE OF CONTENTS

Page

ACKNOWLEDGMENT • • • • • • • • : • • . : •

LIST OF TABLES

LIST OF FIGURES.

Chapter

1. INTRODUCTION • • . . . • • • • • . . 1

2. DETAILS OF THE DISTORTED WAVE APPROXIMATION. 10

1. D.W.A. Transition Amplitude and Cross Section . . . . . . . . . 10

2. Form Factors . . . . . . • . . 12

3. Integration Over Internal Coordinates . 19

4. Final Reduction of Partial Matrix Element . . . . . . . . . 23

5. Zero-Range Interaction . . . . . 33

6. Approximate Treatment of Antisymmetrization . . . . . . . 34

7. Transition Densities..$\quad \cdot \quad \cdot \quad \cdot \quad \cdot \quad \cdot 39$

3. IMPULSE APPROXIMATION PSEUDO-POTENTIAL • • 42

4. THE PROJECTILE-TARGET INTERACTION . . . . 48

5. THE APPROXIMATE TREATMENT OF ANTISYM-

METRIZATION. • • • • • • • . . 58

1. Yukawa Function . . . . . . 59

2. Transitions in $\mathrm{Zr} 90+\mathrm{p} . \quad . \quad . \quad . \quad . \quad 59$

3. Transitions in $\mathrm{C}^{12}, \mathrm{O}^{16}$, and $\mathrm{Ca}^{40}+\mathrm{p}$. 76

4. Summary . . . . . . . . . . 82

5. K-K Force . . . . . . . . . . 83

6. Effective Range Forces : $\cdot$. $:$ : $\quad 88$ 
Chapter

Page

6. STUDY OF INTERACTION MODELS IN D.W.A.

CALCULATION. • • • • • • • • • • 97

1. Section A . . . . . . . . . 98

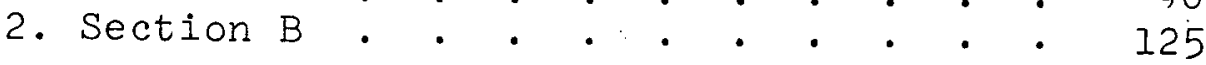

7. CORE POLARIZATION IN INELASTIC PROTON-

NUCLEUS SCATTERING • • • • • • • • • 139

1. Introduction . • • . • • • • 139

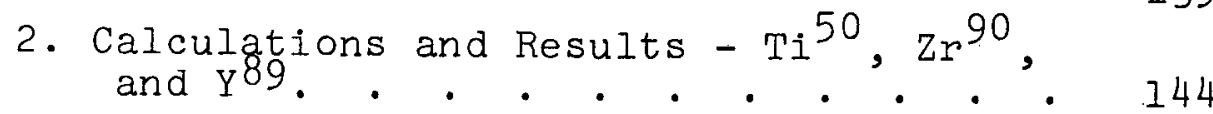

3. Single Proton $1 \mathrm{~h}_{9 / 2^{-1 i_{13 / 2}}}(\mathrm{Q}=-1.61$

MeV) Transition in Bi209. . . . . 209

8. SUMMARY AND CONCLUSIONS • • • • • • • 216

$\operatorname{REFERENCES} \cdot$ • • • • • • • • • • • • • 218

Appendix

A. APPROXIMATE SERIES OF EXCHANGE COMPONENT OF

D.W.A. TRANSITION AMPLITUDE. • • • . 227

B. TRANSITION DENSITIES AND FORM FACTORS. • . 232

1. Harmonic Oscillator Wave Functions . . 232

2. Macroscopic Vibrational Model. . . . 234

3. Reduced Matrix Elements and Transition Densities for Various Transitions. . 239

4. Note on Phases. . . . . . . 247

5. Multipole Coefficients . $\cdot$. $\cdot$. $: 248$

C. INELASTIC ELECTRON-NUCLEUS SCATTERING. • . 250

D. CORE POLARIZATION • . . . . . . . . 257

1. Introduction . • • . • • 257

2. Macroscopic Treatment of Core Polariza- 260

3. Microscopic Treatment of Core Polariza- 260

4. Microscopic Empirical Formula. : $\cdot$ : $: 269$ 


\section{LIST OF TABLES}

Table

Page

2.1 Coefficients for expansion of $t_{S T}^{E}\left(r_{0 I}\right)$ in

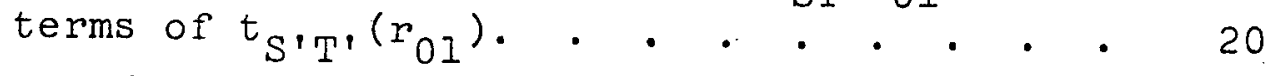

2.2 Interaction components when $i-s p i n$ is not used. • • • • . . . . . . . . . . 29

3.1 Strength and range parameters for components of impulse approximation pseudo-potential . 46

3.2 Strengths for real IF range Yukawa "equivlent" to impulse approximation pseudopotential.

4.1 Comparison of strengths of various real IF range Yukawa "equivalent" interactions.

5.1 Fourier transform of Yukawa interactions of various ranges as a function of the lab energy

5.2 Comparison of approximate and exact values of $\alpha$, the enhancement of the direct cross section due to exchange, for two Yukawa forces with different ranges. . . .

5.3 Approximate energy dependence of $\alpha$ as a function of multipole and range for $a$ Yukawa force

5.4 Strengths of components of exchange interaction. for $\mathrm{K}-\mathrm{K}$ force as a function of the lab energy with the assumption of fixed separation distances... . . . . .

5.5 Same as Table 5.4 except separation distances are allowed to vary with energy ...

5.6 Forces which are consistent with low energy nucleon-nucleon scattering data. 
Table

Page

5.7 Strengths of : components of exchange interaction for Yukawa effective range force as a function of the lab energy. . . . . . 94

6.1 Optical parameters used in $\mathrm{C}^{12}$ and $\mathrm{Ca}^{40}$ calculations . . . . . . . . . . . 117

6.2 Integrated cross sections corresponding to results shown in Fig. 6.6 to Fig. 6.14. Decomposition of $\sigma_{\mathrm{T}}$ into $\sigma_{\mathrm{dir}}$ and $\sigma_{\mathrm{ex}}$ is given for the results obtained with the $K-K$ and Yukawa effective range force . . . . 118

7.1 Extraction of $<\mathrm{k}_{\mathrm{V}}>\theta_{\mathrm{L}}$ from bound state matrix elements of Kuo and Brown . . . . 146

7.2 Composition of core transition densities for $L=2-8$ transitions in $\mathrm{Zr} 90$. . . . 152

7.3 Composition of core transition densities for $\mathrm{L}=2-6$ transitions in Ti50 . . . . 156

7.4 Composition of core transition densities for $L=0$ transition in $\mathrm{Zr} 90$. . . . . 168

7.5 Composition of core transition densities for transition to $Q=-.908 \mathrm{MeV}$ level of $\mathrm{Y}^{89}$. 170

7.6 Optical parameters used in calculating the $\mathrm{Zr}^{90}$, $\mathrm{Ti}^{50}$, and $\mathrm{Y}^{89}$ angular distributions . 177

7.7 Decomposition of integrated cross sections corresponding to results shown in Fig. 7.3 , 7.4 , and 7.5

7.8 Theoretical and experimental values for $\varepsilon_{p}^{2}$ corresponding to the results shown in Fig. $7.3,7.4$, and 7.5 . . . . . 186

7.9 Effective charges for electric $2^{L}$-pole components of transition amplitudes for $\mathrm{Zr} 90$, $\mathrm{Ti}^{50}$, and $\mathrm{Y}^{89}$. . . . . . . 194 
7.10 Experimental and theoretical values for the normalized proton and neutron transition densities for quadrupole transitions in $\mathrm{Zr} 90$,

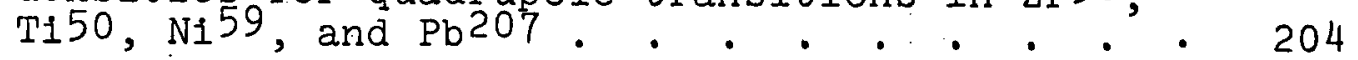

7.11 Normalized proton and neutron transition densities as given by the particle-hole model and particle-hole model with renormalized force for $L=2-8$ transitions in $\mathrm{Zr} 90$ and for $\mathrm{L}=2-6$ transitions in Ti50. Theoretical and experimental enhancement factors are also shown. For $\operatorname{Zr} 90$ the experimental $\varepsilon$ values are from Ref. 67. The Ti50 $\varepsilon$, values are estimates . . 207

7.12 Particle and hole orbitals used in microscopic calculations for $\mathrm{Bi} 209$. 


\section{LIST OF FIGURES}

Figure

Page

3.1 Real part of the $\frac{1}{4}\left(3 \dot{A}_{0}+A_{1}\right)$ component of the free two-nucleon scattering amplitude as a function of q . . . . . . . . . . 44

5.1 Comparison of approximate and exact results showing the variation with energy and interaction range of $\sigma_{\text {ex }} / \sigma_{\text {dir }}$ for several multipoles in the $\mathrm{Zr}^{90}\left(\mathrm{p}, \mathrm{p}^{\prime}\right) \mathrm{Zr}^{90 *}$ reaction . . . . .

5.2 Comparison of $\sigma_{\text {dir }}, \sigma_{e x}$, and $\sigma_{T}$ as function of I for the $m=0.5$ and $3.0 F^{-1}$ cases of Fig. 5.1. Both approximate and exact results are shown for $\sigma_{\mathrm{ex}}$ and $\sigma_{\mathrm{T}} \quad \cdot \quad \cdot \quad \cdot \quad \cdot \quad \cdot$. .

5.3 Exact value of $\sigma_{T}$ for a $2 \mathrm{~F}$ range Yukawa force is compared with $\sigma_{\text {dir }}$ for a $1 F$ and $2 / 3 F$ range Yukawa force as a function of L . • • . . 67

5.4 Direct and approximate exchange angular distributions for $2 F$ range Yukawa force for $L=0$, $2,4,6$, and 8 transitions in $\mathrm{Zr} 90+\mathrm{p}$ at 18.8

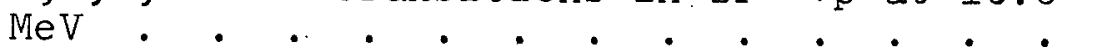

5.5 Direct and exact exchange angular distributions for Yukawa force with range somewhat greater than $1 F$ for $L=2$ transition in $2 r 90$ + p at $18.8 \mathrm{MeV}$. . . . . . . . 70

5.6 Direct and exchange form factors corresponding to results of Fig. 5.2

5.7 Energy dependence of $\sigma_{\text {dir, }} \sigma_{\mathrm{ex}}$, and $\sigma_{\mathrm{T}}$ for $\mathrm{L}=2$ transition in $2 \mathrm{r}^{90}$ calculated with $I F$ range force as in Fig. 5.1 
Figure

Page

5.8 Differential cross sections obtained with the $\mathrm{K}-\mathrm{K}$ "equivalent" interaction for the $L=3$ transition in $\mathrm{Cl}+\mathrm{p}$ at 28.05 and 45.5 $\mathrm{MeV}$. Direct and approximate and exact exchange and total differential cross sections are. shown

5.9 Exact and approximate total differential cross sections calculated with $K-K$ "equivalent" interaction for $\mathrm{L}=2$ transition in $\mathrm{C} I \overline{2}$ $+p$ at $28.05 \mathrm{MeV}$ and $45.5 \mathrm{MeV}$ and for the $\mathrm{L}=3$ and 5 transitions in $\mathrm{Ca} 40+\mathrm{p}$ at 25 and 55 $\mathrm{MeV}$

5.10 Same as Fig. 5.9 for $\mathrm{L}=0$ transition in $\mathrm{C}^{12}+$ $p$ at 28.05 and $45.5 \mathrm{MeV}$ and $\mathrm{L}=3$ transition in $0^{16}+\mathrm{p}$ at $24.5 \mathrm{MeV}$.

5.11 Comparison of Fourier transforms of singlet even and triplet even components of $\mathrm{K}-\mathrm{K}$ force with those of Gaussian, exponential, and Yukawa effective range forces.

5.1' Comparison of exact results obtained with long range part of $\mathrm{H}-\mathrm{J}$ potential with approximate results given by $\mathrm{K}-\mathrm{K}$ force. . . . .

6.1 R.P.A. vector and transition densities for $1^{+} \mathrm{T}=1(\mathrm{Q}=-15.1 \mathrm{MeV})$ level of $\mathrm{C}^{12}$. . . .

6.2 R.P.A. vector, transition densities, with theoretical and experimental inelastic electron scattering form factors for the $2^{+} \mathrm{T}=0(\mathrm{Q}=-4.43 \mathrm{MeV})$ level of $\mathrm{C}^{\mathrm{l}}$

6.3 Same as Fig. 6.2 for $3^{-} \mathrm{T}=\mathrm{O}(\mathrm{Q}=-9.63 \mathrm{MeV})$ level of $\mathrm{Cl}^{12}$. . . . . . . . . . . . 102

6.4 Same as $F_{i g} 6.2$ for $3^{-} \mathrm{T}=0(\mathrm{Q}=-3.73 \mathrm{MeV})$ level of $\mathrm{Ca}^{40}$. . . . . . . . . . . 103

6.5 Same as $\mathrm{Fig}_{40} 6.2$ for $5^{-} \mathrm{T}=0(\mathrm{Q}=-4.48 \mathrm{MeV})$
level of $\mathrm{Ca} . . \quad . \quad . \quad . \quad . \quad . \quad . \quad 104$

6.6 Differential cross sections obtained with impulse approximation pseudo-potential for $\mathrm{L}=0$ transition in $\mathrm{C}^{1}$ 
6.7 Same as Fig. 6.6 for $\mathrm{L}=3$ transition in C12

6.8 Same as Fig. 6.6 for L=2 transition in $\mathrm{C}^{12}$ and $L=3$ and 5 transitions in $\mathrm{Ca}_{40} . . . \quad$. . 110

6.9. Same as Fig. 6.6 for $\mathrm{K}-\mathrm{K}$ force . . . . . 111

6.10 Same as Fig. 6.7 for $\mathrm{K}-\mathrm{K}$ force. Decomposition of complete differential cross sections into direct and exchange components is also shown.

6.11 Same as Fig. 6.8 for $\mathrm{K}-\mathrm{K}$ force . . . . . 113

6.12 Same as Fig. 6.6 for Yukawa effective range force.

6.13 Same as Fig. 6.10 for Yukawa effective range force.

6.14 Same as Fig. 6.8 for Yukawa effective range force. . . . . . . . . . .

6.15 Form factors for $\mathrm{L}=2$ transition in $\mathrm{C}^{12}$ obtained with the $\mathrm{K}-\mathrm{K}$ force and Yukawa effective range force. . . . . . . . .

6.16 Differential cross sections for excitation of first two excited states of $\mathrm{Li}^{6}$ by 24.4 $\mathrm{MeV}$ protons.

6.17 Result obtained with $\mathrm{K}-\mathrm{K}$ force for $\mathrm{L}=2$ transition in $\mathrm{Li}^{6}$ based on empirical transition density determined from inelastic electron scattering data . . . . . . . 128

6.18 Differential cross section for excitation of $2^{+} \mathrm{T}=I(\mathrm{Q}=-16.1 \mathrm{MeV})$ level of $\mathrm{C}^{12}$ by $45.5 \mathrm{MeV}$ protons

6.19 Differential cross sections for excitation of $3^{-T} \mathrm{~T}=0(\mathrm{Q}=6.13 \mathrm{MeV})$ level of 016 by 24.7 $\mathrm{MeV}$ protons 
6.20 Differential cross sections for excitation of $3^{-T}=0(Q=-6,28 \mathrm{MeV})$ and $2^{-T} \mathrm{~T}=0(\mathrm{Q}=-6.02 \mathrm{MeV})$ levels of $\mathrm{Ca}^{40}$ by $24.5 \mathrm{MeV}$ protons... . 134

6.21 Differential cross sections for excitations of $3^{-}(\mathrm{Q}=-2.62 \mathrm{MeV})$ and $5^{-}(\mathrm{Q}=-3.10 \mathrm{MeV})$ level of $\mathrm{Pb} 208$ by 40 and $24.5 \mathrm{MeV}$ protons, respectively

6.22 Differential cross sections for excitation of $1-\mathrm{T}=0$ levels in $\mathrm{C}^{12}$ and $\mathrm{Ca} 40 . .138$

7.1 Spectra of $\mathrm{Ti}^{50}$ and $\mathrm{Zr}^{90}$ showing pairing effect due to core polarization. . . . . 148

7.2 Structure of transition density for $L=0$ transition in $\mathrm{Zr90}$. . . . . . . . . 165

7.3 Differential cross sections for $L=2-8$ transitions in $\mathrm{Zr} 90+\mathrm{p}$ at $18.8 \mathrm{MeV}$. . . 174

7.4 Differential cross sections for $L=2$ and 4 transitions in $\mathrm{Ti} 50+\mathrm{p}$ at 17.5 and $40 \mathrm{MeV}$. 175

7.5 Differential cross sections for excitation of $9 / 2^{+}(\mathrm{Q}=-.908 \mathrm{MeV})$ level in $\mathrm{Y}^{89}+\mathrm{p}$ at $18.9,24.5$, and $61.2 \mathrm{MeV}$. . . . . 176

7.6 Direct form factor and total form factors obtained in microscoplc and macrosgopic calculations for $\mathrm{L}=2$ transition $\mathrm{in} \mathrm{Zr}^{90}+\mathrm{p}$ at $18.8 \mathrm{MeV}$. . . . . . . . . . . 182

7.7 Same as Fig. 7.6 for $\mathrm{L}=2$ transition in $\mathrm{Ti}^{50}+$ $\mathrm{p}$ at $17.5 \mathrm{MeV}$. . . . . . . . . . 183

7.8 Same as Fig. 7.7 for $\mathrm{Ti}^{50}+\mathrm{p}$ at $40 \mathrm{MeV}$. 184

7.9 Differential cross section for $L=0$ transition $\mathrm{Zr}^{90}+\mathrm{p}$ at $12.7 \mathrm{MeV}$. . . . . . . 192 
7.10 Experimental relationship between $\varepsilon_{p}\left(\varepsilon_{n}\right)$ and $e_{p}\left(e_{n}\right)$ for quadrupole transitions in $\mathrm{Zr}^{90}, \mathrm{Ti} 50, \mathrm{Ni} 58$, and $\mathrm{Pb}^{207}$. . . . . 201

7.11 The experimental data compared with the theoretical results obtained with both sets of wave functions. The total differential cross sections and the (303) component are shown for both cases 
CHAPTER I

\section{INTRODUCTION}

There are several factors responsible for the current interest in the microscopic description of inelastic nucleon-. nucleus scattering at medium energies, i.e. incident energies ranging from 15-70 MeV. Most important are recent advances in the theory of nuclear structure which provide a description of a variety of nuclear states in terms of the motions of the individual nucleons which comprise these systems.1,2 The medium energy region is of particular interest primarily because it is the best source of data on these reactions. This is credited to the new sector-focussed cyclotrons and the large tandem accelerators.

Much has been said in the literature about this problem. Ref. 3-7 are a representative sample of papers and a reasonably good bibliography is contained therein. These papers consider some of the formal aspects of the problem and discuss those features of inelastic nucleon-nucleus scattering which make these reactions valuable for studying nuclear structure. Emphasis is on the distorted wave approximation (D.W.A.); however, a good discussion of the coupled channels method is given in Ref. 5. The treatment of the non-local D.W.A. 
transition amplitude ${ }^{\dagger}$ is discussed in Ref. 3. This is encountered when the required antisymmetrization of the projectile-target wave functions is taken into account. The results of several calculations are also available. $8-14$ In these works the local D.W.A. is used and the question of antisymmetrization is ignored. It is assumed that the projectile-target interaction can be expressed as a sum of two-body interactions between the projectile and individual target nucleons. The two-body interaction is taken to be local and scalar, separately in spin, i-spin, and coordinate space. Various radial forms are used and the strength and range parameters are fixed by direct calculation and comparison with experiment. Simple shell model wave functions are used to describe the target nuclei. Application is restricted to the $\left(p, p^{\prime}\right)$ and $(p, n)$ reactions (a limitation imposed by the experimental data) and the transitions considered serve to isolate different components of the interaction. As far as the weak components of the force are concerned, the information extracted in this manner shows some consistency; however, these analyses yield a large range of values for the strength of the strong, non"spin-flip" components of the force. In addition these strengths are considerably larger than that expected from a knowledge of the free two-nucleon force.

In this work the terms local and non-local D.W.A. are used to specify the character of the operator appearing in the D.W.A. transition amplitude, i.e. local or non-local in the projectile coordinate. 
In related calculations the description of the target nuclei is improved so as to introduce explicitly the effects due to core polarization in those transitions which proceed through the strong parts of the interaction. 15,16 The macroscopic vibrational model is used to describe the core and a closure assumption makes it possible to fix the core parameters from experimental $\gamma$-transition rates. The effects are large and much smaller interaction strengths result when they are included. It is likely that core polarization can account for many of the inconsistencies noted in the earlier works. The effects due to antisymmetrization are contained in the exchange component of the transition amplitude which is necessarily non-local. Its properties are presently being investigated. Initial results indicate that it cannot be neglected and that its importance is a function of incident nucleon energy, multipolarity of transition, radial form and exchange nature of the two-body force, and initial and final target states. 17-19 This dependence places restrictions on the two-body interaction and implies a re-evaluation of some of the conclusions obtained in analyses in which antisymmetrization is ignored.

Considerable success has attended the use of "realistic forces" in the bound state problem. 20-25 The theoretical foundations of this approach are reviewed in several places.1,26-29 (Ref. 27 due to MacFarlane is an excellent article.). The major step is this treatment is the introduction of the shell model reaction matrix, or G-matrix, as 
the interaction between bound nucleons. ${ }^{\dagger}$ This is obtained directly from a two-nucleon potential in a manner which takes into account the presence of other nucleons in the nucleus and eliminates the need for using wave functions with short range two-particle correlations. The G-matrix used in Ref. 20-25 is derived from the Hamada-Johnston (H-J) potential which fits the nucleon-nucleon scattering data up to $300 \mathrm{MeV} .30$ Application has been made to nuclei not more than two nucleons away from a closed shell.

The success of this treatment of the bound state problem is very encouraging. Because of its fundamental nature, it avoids many of the difficulties associated with commonly used empirical methods where the interaction is essentially left free. 20 The biggest difficulty is the compensatory relation between the particular calculation which is performed (the proper calculation is, of course, not known a'priori) and the interaction which is so determined. These. remarks need not be confined to the bound state problem. As an example, note that the initial empirical efforts $8-14$ on the inelastic nucleon-nucleus scattering problem conceal the importance of core polarization and antisymmetrization. The purpose of this paper is to explore a parallel treatment of the microscopic description of inelastic nucleon-

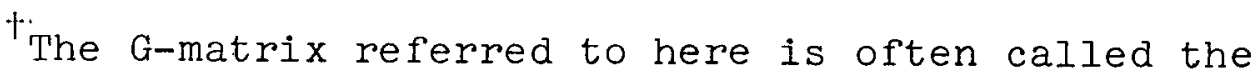
"bare" G-matrix. This provides a means of differentiating between matrix elements of this operator and corresponding matrix elements which implicitly contain effects other than interaction of nucleons through this operator alone, e.g. core polarization effects.
} 
nucleus scattering. Here, asserted a'priori, are three models for the projectile-target interaction. All of these relate directly to the free two-nucleon force. The word models is used because no attempt at a precise derivation of the projectile-target interaction is made. This hopefully can be done within the framework of the many body theory of these reactions in a manner analagous to that followed in the treatment of the bound state problem. In this work the asserted interaction models are simply investigated by direct calculation. In related works they are used to calculate optical potentials for elastic nucleon-nucleus scattering in the medium energy region. 31,32

To be specific, it is assumed that the projectiletarget interaction is given by (i) a pseudo-potential derived from the impulse approximation, (2) the long range part of the Kallio-Kolltveit potential ( $K-K$ force) which is known to be a good approximation to the central part of the shell model reaction matrix, and (3) a Yukawa force derived from effective range theory. These interactions have the same forms, i.e. local, scalar, etc. . ., as those used in previous investigations and all calculations are carried out using the local D.W.A. Any effects due to long range correlations-for example core polarization effects--are included explicitly in the target wave functions. Antisymmetrization is treated approximately in the impulse approximation and the effects are contained implicitly in the pseudo-potential. For the case of the reaction matrix and effective range 
interactions a local approximation to the exchange component of the D.W.A. transition amplitude is included in the calculations.

The impulse approximation ${ }^{33}$ is a free scattering approximation which can be derived from the formal multiple scattering solution to the nucleon-nucleus scattering problem which was developed by watson and collaborators. 34-38 This approximation has been applied with success to inelastic protonnucleus scattering primarily at incident energies greater than $100 \mathrm{MeV} .{ }^{39-44}$ It is generally assumed to be invalid at energies lower than $100 \mathrm{MeV}$; however, there are indications that it might give good results at energies as low as $50 \mathrm{MeV} .36,45$ The pseudo-potential is simply a fit to the Fourier transform of the free two-nucleon scattering amplitude which is calculated from the H-J potential, of $f$ the energy shell, i.e. using nucleon-nucleus kinematics in place of nucleon-nucleon kinematics.

The Kallio-Kolltveit potential contains a hard core and has an exponential radial form. 46 It fits the nucleonnucleon S-wave phase shifts up to $300 \mathrm{MeV}$. The long range part of this potential is defined by the Scott-Moskowsi separation method, 47 i.e. a separation distance is determined (it turns out to be of the order of $1 F$ ) below which the potential is set to zero. The separation method gives the leading term in a perturbation expansion for the components of the reaction matrix which act in states of even relative orbital angular momentum. This force is a good approximation 
to the central part of the G-matrix used in Ref. 20-24. The latter is derived from a more complete potential and contains additional detail. Application of the $K-K$ force to the calculation of the low energy spectrum of $0^{16}$ in Ref. 46 was one of the first attempts to use "realistic forces" in the bound state problem. In evaluating bound state matrix elements it is assumed that the $K-K$ force acts only in relative s-states.

The impulse approximation pseudo-potential and $\mathrm{K}-\mathrm{K}$ force are selected because it is possible that they are valid representations of the projectile-target interaction asymptotically, i.e. far outside and deep inside the nucleus, respectively. Reference to the high energy features of nucleon-nucleon scattering is contained in the potentials from which they are derived. It is of interest to see how these interaction models differ from the forces of regular functional form which are obtained in the shape independent analysis of low energy nucleon-nucleon scattering data. 48,49 To this end calculations are performed with a Yukawa effective range force. Consideration by way of discussion is also given to Gaussian and exponential effective range interactions. There is an imaginary division of the remainder of this paper into two parts. Details relating to the interaction models, D.W.A. calculations, and exchange approximation are contained in Chapters $2-5$ and a few Appendices. Applications and results are presented in Chapters 6 and 7 with Chapter 8 reserved for final remarks. To be a bit more specific, a 
discussion of the antisymmetrized D.W.A. is given in Chapter 2. The approximation used to treat exchange is also developed here. The impulse approximation pseudo-potential is presented in Chapter. 3. Chapter 4 contains some rough arguments concerning the possible character of the actual projectiletarget interaction and its relation to the $\mathrm{K}-\mathrm{K}$ force and impulse approximation pseudo-potential. The effective range forces are introduced in Chapter 5 where some of the properties of the "approximate" exchange component of the D.W.A. transition amplitude are discussed and a few results obtained with exchange treated approximately are compared with results of exact calculations. 18,19 At this point the $K-K$ force and effective range forces are compared on the basis of this approximation.

Applications, mainly to $\left(p, p^{\prime}\right)$ transitions in closed and pseudo-closed shell nuclei, i.e. $\mathrm{C}^{12}, \mathrm{O}^{16}, \mathrm{Ca}^{40}$, and $\mathrm{Pb}^{208}$, are considered in Chapter 6. Random phase approximation (R.P.A.) state vectors are used to describe the target nuceli. ${ }^{50-54}$ Studies of the $\left(p, p^{\prime}\right)$ reaction at incident energies above $100 \mathrm{MeV}^{40-44^{\prime}}$ and studies of the $\left(e, e^{\prime}\right)$ reaction 55,56 indicate that these vectors give a good description of the transitions considered. These transitions serve to test the proposed interaction models at least to within the quality of the approximation used to treat antisymmetrization. Some inelastic electron scattering results are presented in order to provide a frame of reference for examining the $\left(p, p{ }^{\prime}\right)$ differential cross sections which are presented. 
Chapter 7 is devoted to the treatment of transitions involving low lying states in nuclei which possess one or two nucleons outside of a closed shell. Core polarization plays an important part in these transitions 15,16 and has an equally important effect on the relative spacing of these low lying leveis. ${ }^{20-25}$ The effects of core polarization are estimated in calculations which use either a microscopic model or the macroscopic vibrational model to describe the core. Emphasis is on the completely microscopic calculations which assume that the core can be described by a zero-order shell model Hamiltonian and that only the effect of simple particle-hole excitations up to roughly $\$ \omega$ in energy need be considered. The coupling between the valence nucleons and the core is treated by first order perturbation theory and the $K-K$ force is taken to be the coupling interaction. This is essentially the model first used by Horle and Arima in calculating quadrupole moments ${ }^{57}$ and it is the same picture that Kuo and Brown have used in Ref. 20-25. Differential cross sections for ( $\left.p, p^{\prime}\right)$ transitions and $\gamma$-transition rates are calculated. For the most part, the $K-K$ force is used for the projectile-target interaction. The completely microscopic $\left(p, p^{\prime}\right)$ calculations are amusing as they constitute a first attempt to calculate the observed cross sections directly from a knowledge of the two-nucleon force. The relation between the effect of core polarization on the spectrum and in transitions is examined. Conclusions are drawn as to the validity of the particle-hole model. 


\section{CHAPTER 2}

\section{DETAILS OF THE DISTORTED WAVE APPROXIMATION}

1. D.W.A. Transition Amplitude and Cross Section The antisymmetrized distorted wave transition amplitude for the inelastic nucleon-nucleus scattering reaction $\bar{k}_{a} a, A \rightarrow \bar{k}_{b} b, B$. (where $\bar{k}$ is the relative momentum of the target and projectile, the small letters represent the internal projectile quantum numbers, and the capital letters are used to specify the state of the target) is given by $3,16,17$

$$
\begin{aligned}
\mathrm{T}_{\mathrm{DW}} & =\left\langle\mathrm{B}\left|\sum_{\mathrm{rp}} \mathrm{a}_{\mathrm{p}}^{+} \mathrm{a}_{\mathrm{r}}\right| \mathrm{A}\right\rangle \\
& \mathrm{x}_{\mathrm{m}_{\mathrm{a}}^{\prime} \mathrm{m}_{\mathrm{b}}^{-}}<\mathrm{x}_{\mathrm{m}_{\mathrm{b}}^{\prime} \mathrm{m}_{\mathrm{b}}}^{(-)}(0) \phi_{\mathrm{p}}(1)|\mathrm{t}(0,1)| \mathrm{x}_{\mathrm{m}_{\mathrm{a}}^{\prime} \mathrm{m}_{\mathrm{a}}}^{(+)}(0) \phi_{\mathrm{r}}(1)-\mathrm{x}_{\mathrm{m}_{\mathrm{a}}^{\prime} \mathrm{m}_{\mathrm{a}}}^{(+)}(1) \phi_{\mathrm{r}}(0)>
\end{aligned}
$$

where a local interaction model is implied and provision is made for the presence of spin-orbit coupling in the optical potential. In this expression $t(0,1)$ denotes the two-body interaction, the $x^{\prime}$ s are the distorted waves which describe the relative motion of the projectile and target under the influence of the optical potential, and co-factor expansions of the target wave functions are employed. The latter account for the presence of the creation (destruction) operators $\mathrm{a}^{+}(\mathrm{a})$ and the single particle bound state wave functions $\phi$ in the relation. The arguments 
0 and $I$ refer to all nucleon coordinates and fix the manner in which the integrals are to be taken.

The first integral in Eq. (1) is the usual direct matrix element while the second is the exchange component of the transition amplitude. In the former the same particle is unbound in both the initial and final states, but in the latter the particle which is unbound initially is captured into the nucleus and a target particle is expelled into the final unbound state.

The distorted waves are solutions to a one body Schrodinger equation which contains the optical potential. Spin projection is not a good quantum number when this potential contains a spin-orbit term. In Eq. (1) $x_{\mathrm{m}_{\mathrm{a}} \mathrm{m}_{\mathrm{a}}}$ $\left(x_{m_{b}^{\prime}} m_{b}\right)$ is the $m_{a}^{\prime}\left(m_{b}^{\prime}\right)$ spin projection component of the solution with initial spin projection $m_{a}\left(m_{b}\right)$. It is clear that spin orbit coupling gives rise to "spin-flip" in inelastic scattering over and above that which occurs through direct interaction via $t$. Note further the standard use of the superscripts on the distorted waves to specify the boundary conditions which they satisfy.

From the form of the transition amplitude it is seen that inelastic nucleon-nucleus scattering is represented by a one-body operator in the distorted wave approximation, i.e. transitions are allowed between components of the target wave functions which differ only in the state of a single nucleon. A state of the target nucleus is defined by its total angular momentum, projection, and additional quantum numbers; the internal state of the projectile nucleon 
is fixed by giving its spin and 1 -spin projection; thus $A \equiv \alpha_{A} J_{A} M_{A}, \quad a \equiv m_{a} \tau_{a}, \quad B \equiv \alpha_{B} J_{B} M_{B}$, and $b \equiv m_{b} \tau_{b}$. Further $a^{\prime}$ and $b^{\prime}$ will be used for $m_{a}^{\prime} \tau$ and $m_{b}^{\prime} \tau_{b}$, respectively.

Scattering experiments are most frequently performed with unpolarized beams and targets. Under these conditions the differential cross section for inelastic nucleon-nucleus scattering is obtained by introducing kinematical factors and appropriately summing and averaging over projections. This gives

$$
\frac{d \sigma}{d \Omega}=\left(\frac{\mu}{2 \pi \varkappa^{2}}\right)^{2} \frac{k_{b}}{k_{a}} \frac{l}{2\left(2 J_{A}+I\right)} \sum_{\substack{M_{A} M_{B} \\ m_{a} m_{b}}}\left|T_{D W}\right|^{2}
$$

where $\mu$ is the reduced mass of the projectile-nucleus system.

\section{Form Factors}

Without loss of generality the $j-j$ coupling representation can be selected as the single particle basis, ie. $p=n^{\prime} \ell^{\prime} j^{\prime} m^{\prime} \tau^{\prime}$ and $r=n \ell j m \tau$, which gives

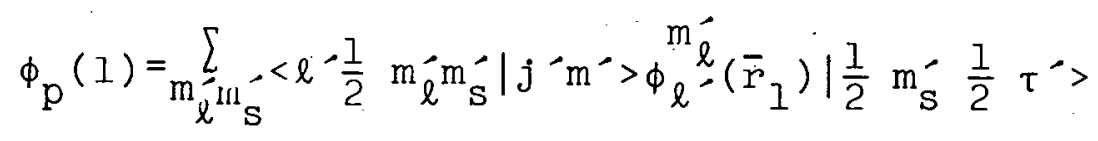
$\phi_{r}(I)=\sum_{m_{l} m_{S}}\left\langle l \frac{I}{2} m_{\ell} m_{S}\left|j m>\phi_{\ell}^{l}\left(\bar{r}_{I}\right)\right| \frac{1}{2} m_{s} \frac{1}{2} \tau\right\rangle$

where $\langle a b \alpha \beta \mid c \gamma\rangle$ is a Clebsch-Gordan coefficient. ${ }^{58}$ It is convenient to rewrite $\mathrm{Eq}$. (I) as 


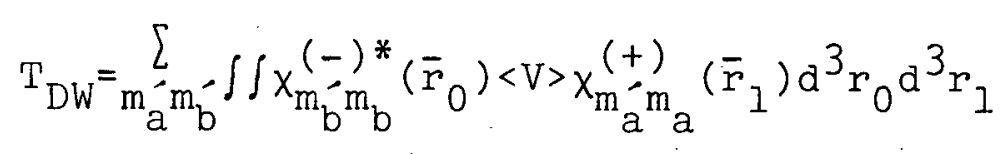

where

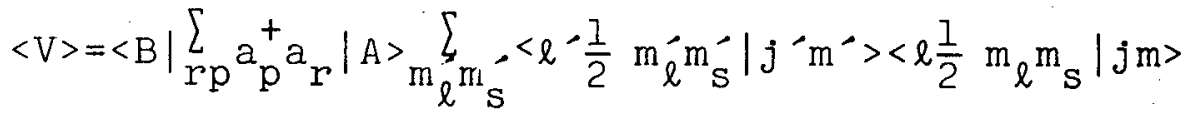

$$
\begin{aligned}
& m_{\ell} m_{s} \\
& x\left\{\delta\left(\bar{r}_{0}-\bar{r}_{1}\right) \int \phi_{\ell}^{m_{\ell}^{\prime *}}\left(\bar{r}_{2}\right)<b ; p|t(0,2)| a ; r>\phi_{\ell}{ }^{l}\left(\bar{r}_{2}\right) d^{3} r_{2}\right. \\
& \left.-\phi_{\ell}^{m^{\prime} \ell^{*}}\left(\bar{r}_{1}\right)<b ; p|t(0,1)| r, a^{-}>\phi_{\ell}{ }^{\ell}\left(\bar{r}_{0}\right)\right\}
\end{aligned}
$$

with the bra-ket notation applying to integration over the internal coordinates only.

The quantity $\langle V\rangle$, called the partial matrix element, contains all of the nuclear structure information for a particular transition. It also contains the details of the interaction model and the selection rules which govern the reaction. It is an effective one-body operator in the projectile subspace. Examination of Eq. (5) shows that $\langle\mathrm{V}\rangle$ is non-local, that is it depends on $\bar{r}_{0}$ and $\bar{r}_{1}$. Here this non-locality arises due to the presence of the exchange component of the transition amplitude; however, had $t$ been assumed non-local the direct component of the transition amplitude would also contribute to the non-locality in $\langle V\rangle$.

The general rotational properties of the partial matrix element can be exploited to reduce the distorted wave calculations for all transitions to a common form. It can be shown quite generally that $\langle\mathrm{V}\rangle$ can be written ${ }^{3}$ 


$$
\begin{aligned}
<V>= & \sum_{L S^{\prime} i^{1}}-L_{F_{L S J}, M^{\prime}}\left(\bar{r}_{0}, \bar{r}_{I} ; b^{\prime} B ; a^{\prime} A\right)(-1)^{1 / 2-m_{b}^{\prime}}<J_{A} J M_{A}, M_{B}-M_{A} \mid J_{B} M_{B}> \\
& x<\frac{1}{2} \frac{1}{2} m_{a}^{\prime},-m_{b}^{\prime}\left|S m_{a}^{\prime}-m_{b}^{\prime}><L S M, m_{a}^{\prime}-m_{b}^{\prime}\right| J, M_{B}-M_{A}>
\end{aligned}
$$

where $\vec{N}=\mathbb{M}_{B}-M_{A}+m_{b}^{\prime}-m_{a}^{\prime} \cdot \quad F_{L S J}, \mathbb{M}$ transforms under rotation of the coordinate system as $\mathrm{Y}_{L M}^{*}$, the $i^{-L}$ insures convenient time reversal properties, and $L, S$, and $J$ satisfy the vector relations

$$
\bar{J}=\bar{J}_{B}-\bar{J}_{A} \quad \bar{S}=\bar{S}_{a^{\prime}}^{\prime}-\bar{s}_{b^{\prime}}^{\prime}+S=0, I \quad \bar{L}=\bar{J}-\bar{S} .
$$

It is clear that $\overline{\mathrm{L}}, \overline{\mathrm{S}}$, and $\overline{\mathrm{J}}$ are the angular momenta transferred to the target nucleus through $t$ in the inelastic collision. If $i-s p i n$ is considered to be a good quantum number for the target nucleus $\mathrm{Eq}$. (6) can be rewritten as

$$
\begin{aligned}
& \langle V\rangle=\sum_{L S J T^{i}}-L_{F_{L S J}^{T}, M}\left(\bar{r}_{0}, \bar{r}_{I} ; a^{\prime} A, b^{\prime} B\right)(-I)^{I / 2-m_{b}^{\prime}}<J J_{A} M_{A}, M_{B}-M_{A} \mid J_{B} M_{B}> \\
& \mathrm{x}<\frac{1}{2} \frac{1}{2} \mathrm{~m}_{a}^{-}, \mathrm{m}_{\mathrm{b}}^{-}\left|\mathrm{Sm} \mathrm{m}_{\mathrm{a}}^{-}-\mathrm{m}_{\mathrm{b}}^{-}><\mathrm{LSM}, \mathrm{m}_{\mathrm{a}}^{-}-\mathrm{m}_{\mathrm{b}}^{-}\right| \mathrm{J}, \mathrm{M}_{\mathrm{B}}-\mathrm{M}_{A}> \\
& \mathrm{x}<\mathrm{T}_{\mathrm{A}} \mathrm{TM}_{\mathrm{T}_{\mathrm{A}}}, \mathrm{M}_{\mathrm{T}_{\mathrm{B}}}-\mathrm{M}_{\mathrm{T}_{\mathrm{A}}}\left|\mathrm{T}_{\mathrm{B}} \mathrm{M}_{\mathrm{T}_{\mathrm{B}}}><\frac{1}{2} \mathrm{~T} \tau_{\mathrm{b}}, \tau_{\mathrm{a}}-\tau_{\mathrm{b}}\right| \frac{1}{2} \tau_{\mathrm{a}}>
\end{aligned}
$$

where $\bar{T}=\bar{T}_{B}-\bar{T}_{A}=\bar{t}_{a}-\bar{t}_{b} \rightarrow T=0,1$ and $M_{T_{B}}-M_{T_{A}}=\tau_{a}-\tau_{b}$. Eq. (8) reduces to Eq. (6) by defining.

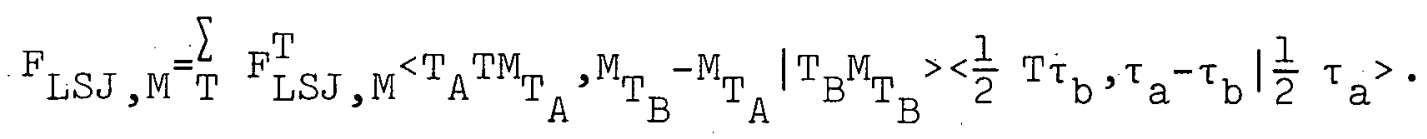

This expansion of the partial matrix element can be used to write the transition amplitude as 


$$
T_{D W}=\sum_{L S J} \hat{J}<J_{A} J M_{A}, M_{B}-M_{A} \mid J_{B} M_{B}>B_{S J} L_{b} m_{a}
$$

where $\hat{J}=[2 J+1]^{1 / 2}$ and

$$
\begin{aligned}
& { }_{B_{S J}}^{L M m_{b} m_{a}}=\sum_{\substack{m_{a} \\
M^{-}}} m_{b}^{-\frac{i^{-L}}{\hat{J}}}(-1)^{1 / 2-m_{b}^{\prime}}<L S M ; m_{a^{\prime}}-m_{b}^{\prime} \mid J, M-m_{b}+m_{a}> \\
& \mathrm{x}<\frac{1}{2} \frac{1}{2} \mathrm{~m}_{\mathrm{a}}^{\prime},-\mathrm{m}_{\mathrm{b}}^{\prime} \mid S \mathrm{~s}_{\mathrm{a}}^{\prime}-\mathrm{m}_{\mathrm{b}}^{\prime}> \\
& x \iint x_{m_{b} m_{b}}^{(-) *}\left(\bar{r}_{0}\right) F_{L S J, M}-x_{m_{a}^{\prime} m_{a}}^{(+)}\left(\bar{r}_{1}\right) d^{3} r_{0} d^{3} r_{1} \text {. }
\end{aligned}
$$

The cross section, Eq. (2), then becomes

$$
\frac{d \sigma}{d \Omega}=\left(\frac{\mu}{2 \pi k^{2}}\right)^{2} \frac{k_{b}}{k_{a}} \frac{2 J_{B}+1}{2(2 J A} \sum_{\substack{J M \\ m_{a}}}\left|\sum_{L S} \beta_{S J}^{L M m_{b} m a}\right|^{2}
$$

with the interference between different $S$ and $L$ for a given $J$ occuring as a direct consequence of the spin-orbit coupling in the optical potential. In practice this interference is found to be weak. As partial wave expansions of the distorted waves are used in evaluating the integral in Eq. (11) the multipole components of $F_{L S J, M}\left(\bar{r}_{0}, \bar{r}_{1}\right)$ are needed. They are defined as follows:

$$
\begin{aligned}
& \mathrm{F}_{L S J, M}\left(\bar{r}_{0}, \bar{r}_{I}\right)=\sum_{L_{a} L_{b}} F_{M_{a} M_{b}}^{S J} \dot{L}_{a} L_{b}\left(r_{0}, r_{I}\right) Y_{L_{b} M_{b}}^{*}\left(\hat{r}_{0}\right) Y_{L_{a} M_{a}}^{*}\left(\hat{r}_{I}\right) \\
& x<L_{b} L_{a} M_{b} M_{a} \mid L M>
\end{aligned}
$$




$$
\begin{aligned}
& \mathrm{F}_{\mathrm{LL}_{b} \mathrm{~L}_{a}}^{\mathrm{SJ}}\left(\mathrm{r}_{0}, \mathrm{r}_{I}\right)=\sum_{\mathrm{M}_{\mathrm{b}} \mathrm{M}_{\mathrm{a}}}\left\langle\mathrm{L}_{\mathrm{b}} \mathrm{L}_{a} \mathrm{M}_{\mathrm{b}} \mathrm{M}_{\mathrm{a}} \mid \mathrm{LM}\right\rangle \\
& x \iint F_{L S J, M}\left(\bar{r}_{0}, \bar{r}_{I}\right) Y_{L_{b} M_{b}}\left(\hat{r}_{0}\right) Y_{L_{a} M_{a}}\left(\hat{r}_{I}\right) d \Omega_{0} d \Omega_{I}
\end{aligned}
$$

The reduction has been achieved. All of the "physics" for a particular transition is contained in the $\mathrm{F}_{\mathrm{LL}_{\mathrm{b}} \mathrm{L}_{a}}^{\mathrm{SJ}}\left(\mathrm{r}_{0}, \mathrm{r}_{1}\right)$ which are independent of projection quantum numbers and are functions of the radial coordinates $r_{0}$ and $r_{1}$. Given these quantities the distorted wave cross section is obtained by computing and summing the $\beta_{\mathrm{SJ}}^{\mathrm{LMm}_{\mathrm{b}} \mathrm{m}_{\mathrm{a}}}$ as prescribed by Eq. (II) and Eq. (12). Unfortunately, the calculation is still not easy. It will be seen that each of these multipole components is a fairly complicated quantity as far as computation'is concerned. Further, $\mathrm{F}_{\mathrm{LL}_{\mathrm{b}} \mathrm{L}_{\mathrm{a}}}^{\mathrm{SJ}}\left(\mathrm{r}_{0}, \mathrm{r}_{1}\right)$ is associatied with angular momentum transfer $\mathrm{L}, \mathrm{S}$, and $\mathrm{J}$ to the target nucleus with the projectile undergoing a transition from the state of relative angular momentum $L_{a}$ to $L_{b}$. Even though only $a$ few values of $\mathrm{L}, \mathrm{S}$, and $\mathrm{J}$ are expected to contribute to a transition there may be as many as twenty partial waves used in the calculation of a cross section in the energy region of interest here. The point is that the $\mathrm{F}_{\mathrm{LL}_{\mathrm{b}} \mathrm{L}_{a}}^{\mathrm{SJ}}\left(\mathrm{r}_{0}, \mathrm{r}_{I}\right)$ are not only complicated, but many of them are required.

For the direct, or local component of the partial matrix element an additional separation can be made.

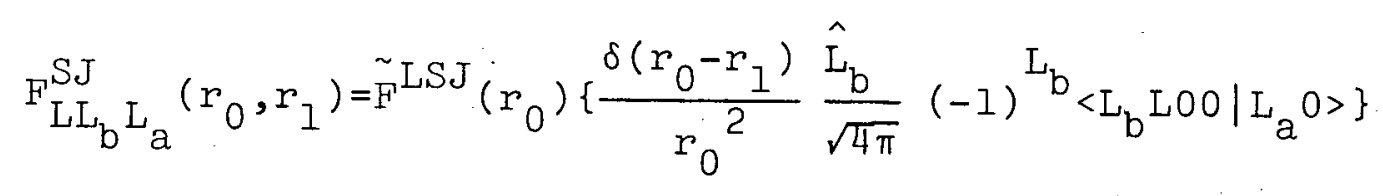


Using result (15) in Eq. (13), recoupling a spherical harmonic, and using the closure property of spherical harmonics gives for this case

$$
\mathrm{F}_{\mathrm{LSJ}, M}\left(\bar{r}_{0}, \overline{\mathrm{r}}_{1}\right)=\tilde{F}^{\mathrm{LSJ}}\left(\mathrm{r}_{0}\right) \mathrm{Y}_{\mathrm{LM}}^{*}\left(\hat{\mathrm{r}}_{0}\right) \delta\left(\overline{\mathrm{r}}_{0}-\overline{\mathrm{r}}_{1}\right)
$$

When Eq. (16) can be used the calculation of the cross section is considerably easier because the "physics" is then contained in the $\tilde{F}^{L S J}\left(r_{0}\right)$ which are few in number and depend on only one radial coordinate. In addition explicit use of Eq. (16) in Eq. (II) gives an expression for $\beta_{S J}{ }_{S J} m_{a}$ which is much simpler than the one obtained by using Eq. (13) in Eq. (11). Computational difficulties associated with the treatment of non-local partial matrix elements have been the major reason for neglecting the effects due to antisymmetrization in the past. Fortunately, this problem is well on its way to solution. 16,17

In this work an attempt is made to account approximately for antisymmetrization in an expression of the form (16). The calculations are then essentially reduced to constructing the form factors $\tilde{F}^{\mathrm{LSJ}}\left(\mathrm{r}_{0}\right) .^{+}$These have two components - $\tilde{D}^{L S J}\left(r_{0}\right)$ which comes from the direct component of the transition amplitude and $\tilde{E}^{L S J}\left(r_{0}\right)$ which approximately represents terms coming from the exchange component.

$$
\text { It is } \mathrm{F}_{\text {LSJ }, M}\left(\bar{r}_{0}, \overline{\mathrm{r}}_{1}\right) \text { which is properly referred to as }
$$
a form factor. When using the local D.W.A. $\tilde{F}^{\mathrm{LSJ}}\left(\mathrm{r}_{0}\right)$ is the essential part of $\mathrm{F}_{\mathrm{LSJ}, \mathrm{M}}\left(\overline{\mathrm{r}}_{0}, \overline{\mathrm{r}}_{1}\right)$. In this work the term form factor will refer to $\tilde{F}^{\text {LSJ }}\left(r_{0}\right)$. 
Two approximations are used--one with the impulse approximation and another for the case of the $K-K$ and effective range forces. The approximations differ only in detail-not in spirit. They are discussed in section 6 of this chapter. Explicit identification of $\tilde{E}^{L S J}\left(r_{0}\right)$ is made when using the $K-K$ and effective range forces, whereas it is implicit in the impulse approximation pseudo-potential. Returning to the discussion of the complete partial matrix element Eq. (13) is used to rewrite Eq. (6) as

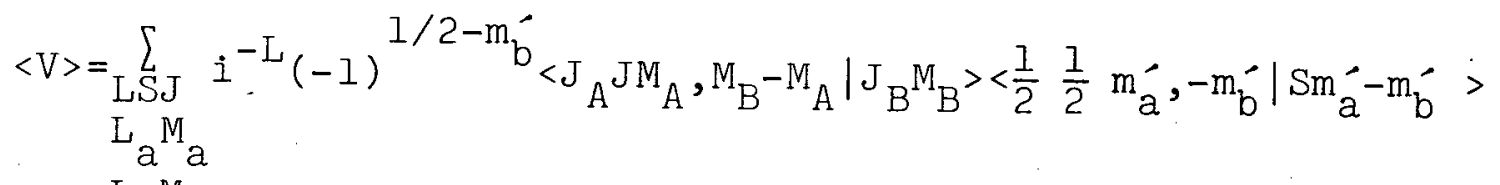

$$
\begin{aligned}
& \mathrm{L}_{b} \mathrm{M}_{\mathrm{b}} \\
& \mathrm{x}<\mathrm{LSM}, \mathrm{m}_{\mathrm{a}}^{\prime}-\mathrm{m}_{\mathrm{b}}^{\prime}\left|\mathrm{J}, \mathrm{M}_{\mathrm{B}}-\mathrm{M}_{\mathrm{A}}><\mathrm{L}_{\mathrm{b}} \mathrm{L}_{a} \mathrm{M}_{\mathrm{b}} \mathrm{M}_{\mathrm{a}}\right| \mathrm{LM}> \\
& x Y_{L_{b} M_{b}}^{*}\left(\hat{r}_{0}\right) Y_{L_{a} M_{a}}^{*}\left(\hat{r}_{I}\right) F_{L L_{b} L_{a}}^{S J}\left(r_{0}, r_{I}\right) \text {. }
\end{aligned}
$$

In the next two sections of this chapter it will be shown that $\mathrm{Eq}$. (5) can be written in the above form, thus allowing identification of the $\mathrm{F}_{\mathrm{LL}_{\mathrm{b}} \mathrm{L}_{\mathrm{a}}}^{\mathrm{SJ}}\left(\mathrm{r}_{0}, \mathrm{r}_{I}\right)$. The discussion is restricted to the static interactions being considered in this work. These have the form

$$
\begin{aligned}
& t(0,1)=t_{00}\left(r_{01}\right)+t_{01}\left(r_{01}\right) \bar{\sigma}_{0} \cdot \bar{\sigma}_{I}+t_{10}\left(r_{01}\right) \bar{\tau}_{0} \cdot \bar{\tau}_{1}+t_{11}\left(r_{01}\right) \bar{\sigma}_{0} \cdot \bar{\sigma}_{1} \bar{\tau}_{0} \cdot \bar{\tau}_{1} \\
& =\sum_{\substack{S^{\prime} \mathrm{I} \\
\lambda \mathrm{y}}}(-1)^{\lambda+\mathrm{y}} \mathrm{t}_{\mathrm{ST}}\left(\mathrm{r}_{01}\right) \sigma_{-\lambda}^{\mathrm{S}}(0) \sigma_{\lambda}^{\mathrm{S}}(1) \tau_{-\mathrm{y}}^{\mathrm{T}}(0) \tau_{\mathrm{y}}^{\mathrm{T}}(I)
\end{aligned}
$$

where $\sigma_{\lambda}^{\mathrm{S}}\left(\tau_{\mathrm{y}}^{\mathrm{T}}\right)$ are the usual spherical tensor components of the $\operatorname{spin}(i-\operatorname{spin})$ operator and $\sigma_{0}^{0}=\tau_{0}^{0}=1$. 


\section{Integration Over Internal Coordinates}

Using Eq. (18) the following result is obtained for the integrals over internal nucleon coordinates in Eq. (5)

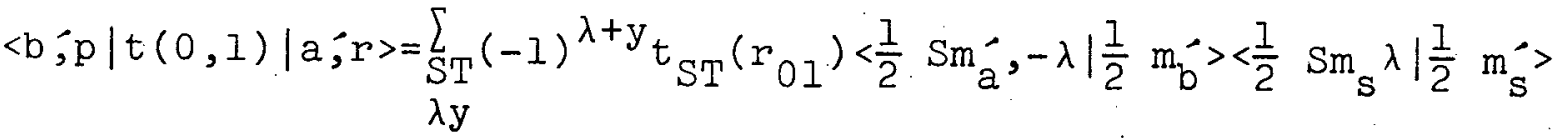

$$
\begin{aligned}
& \mathrm{x}<\frac{1}{2} \mathrm{~T} \tau_{\mathrm{a}},-\mathrm{y}\left|\frac{1}{2} \tau_{\mathrm{b}}><\frac{1}{2} \mathrm{~T} \tau \mathrm{y}\right| \frac{1}{2} \tau^{-}> \\
& x\left\{<\frac{1}{2}|| \sigma^{S}|| \frac{1}{2}><\frac{1}{2}|| \tau^{T}|| \frac{1}{2}>\right\}^{2}
\end{aligned}
$$

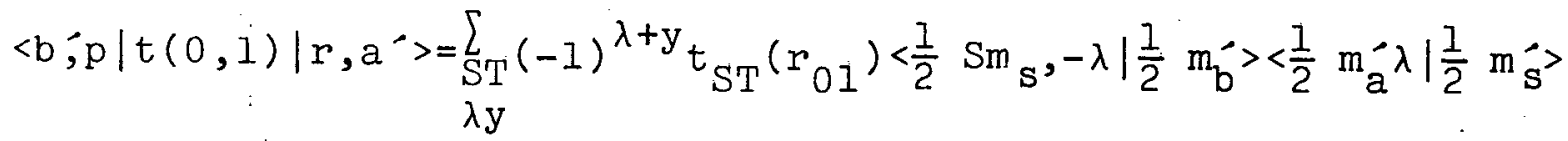

$$
\begin{aligned}
& x<\frac{1}{2} T \tau,-y\left|\frac{1}{2} \tau_{b}><\frac{1}{2} T \tau a^{T}\right| \frac{1}{2} \tau^{\prime}> \\
& x\left\{<\frac{1}{2}|| \sigma^{S}|| \frac{1}{2}><\frac{1}{2}|| \tau^{T}|| \frac{1}{2}>\right\}^{2}
\end{aligned}
$$

where $\left\langle a \alpha\left|O_{b \beta}\right| c \gamma\right\rangle=\langle\operatorname{cb} \gamma \beta \mid a \alpha\rangle\left\langle a|| O_{b}|| b\right\rangle$ is the convention adopted for the Wigner-Eckart Theorem. 58 The following recoupling identity

$$
\begin{aligned}
& <\frac{1}{2} \mathrm{Sm}_{\mathrm{S}},-\lambda\left|\frac{1}{2} \mathrm{~m}_{\mathrm{b}}^{-}><\frac{1}{2} \mathrm{Sm}_{\mathrm{a}}^{-} \lambda\right| \frac{1}{2} \mathrm{~m}_{\mathrm{S}}^{-}>={ }_{\mathrm{S}^{\prime}} \sum_{\lambda}-\hat{S}^{-2} \mathrm{~W}\left(\frac{1}{2} \frac{1}{2} \frac{1}{2} \frac{1}{2} ; \mathrm{SS}^{\prime}\right) \\
& x(-1)^{S^{\prime}-S+\lambda^{\prime}+\lambda+1}<\frac{1}{2} S^{\prime} m_{a}^{\prime},-\lambda^{\prime}\left|\frac{1}{2} m_{b}^{\prime}><\frac{1}{2} S^{\prime} m_{s} \lambda^{\prime}\right| \frac{1}{2} m_{s^{\prime}}^{\prime}>
\end{aligned}
$$

and its i-spin counterpart is used in Eq. (20). Then the fact that

$$
\left\langle\frac{1}{2} \|\left(\dot{\sigma}_{\tau}^{S}\right)|| \frac{1}{2}\right\rangle=(\underset{T}{\hat{S}})
$$

is used and summation indices are interchanged to give 


$$
\begin{aligned}
& 20
\end{aligned}
$$

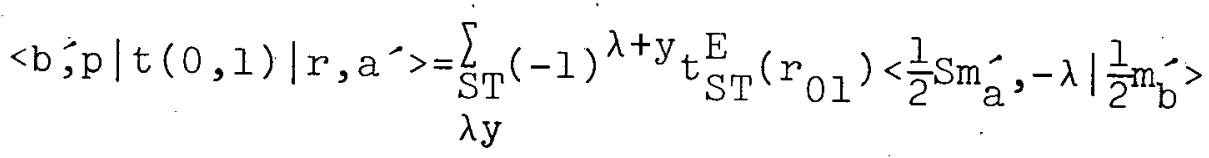

$$
\begin{aligned}
& \mathrm{x}<\frac{1}{2} \mathrm{Sm}_{\mathrm{s}} \lambda\left|\frac{1}{2} \mathrm{~m}_{\mathrm{s}}^{-}><\frac{1}{2} \mathrm{~T} \tau_{\mathrm{a}},-\mathrm{y}\right| \frac{1}{2} \tau_{\mathrm{b}}> \\
& \mathrm{x}<\frac{1}{2} \mathrm{~T} \tau \mathrm{y} \mid \frac{1}{2} \tau^{-}>\left\{\left\langle\frac{1}{2}|| \sigma^{\mathrm{S}}|| \frac{1}{2}\right\rangle\left\langle\frac{1}{2}|| \tau^{\mathrm{T}}|| \frac{1}{2}\right\rangle\right\}^{2}
\end{aligned}
$$

where

$$
\begin{aligned}
& t_{S T}^{E}\left(r_{0 I}\right)=S_{S} T^{r}(-1) S-S+T^{\prime}-T+S^{-2} \hat{T}^{-2} W\left(\frac{1}{2} \frac{1}{2} \frac{1}{2} \frac{1}{2} ; S^{-} S\right) W\left(\frac{1}{2} \frac{1}{2} \frac{1}{2} \frac{1}{2} ; T^{-T}\right) \\
& x_{S^{-} T^{-}}-\left(r_{01}\right)
\end{aligned}
$$

with W indicating a kacah coefficient. 58 . (22) can be summarized as $\left\langle b^{\prime}, p|t(0,1)| r, a^{\prime}\right\rangle=\left\langle b^{\prime}, p\left|t^{E}(0,1)\right| a^{\prime}, r\right\rangle$.

The coefficients in the expansion of $t_{S T}^{E}\left(r_{01}\right)$ in terms of $t_{\mathrm{ST}}\left(r_{01}\right)$ which is given in Eq. (23) will be called $\mathrm{AS}_{\mathrm{ST}} \mathrm{T}^{\prime}$,

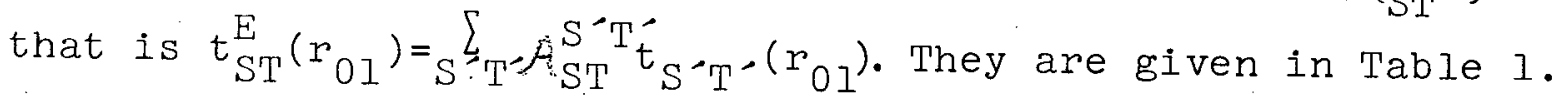

TABLE 1.--Coefficients for expansion of $t_{\mathrm{ST}}^{\mathrm{E}}\left(\mathrm{r}_{\mathrm{Ol}}\right)$ in terms of $t_{S^{\prime} T^{\prime}}-\left(r_{01}\right)$.

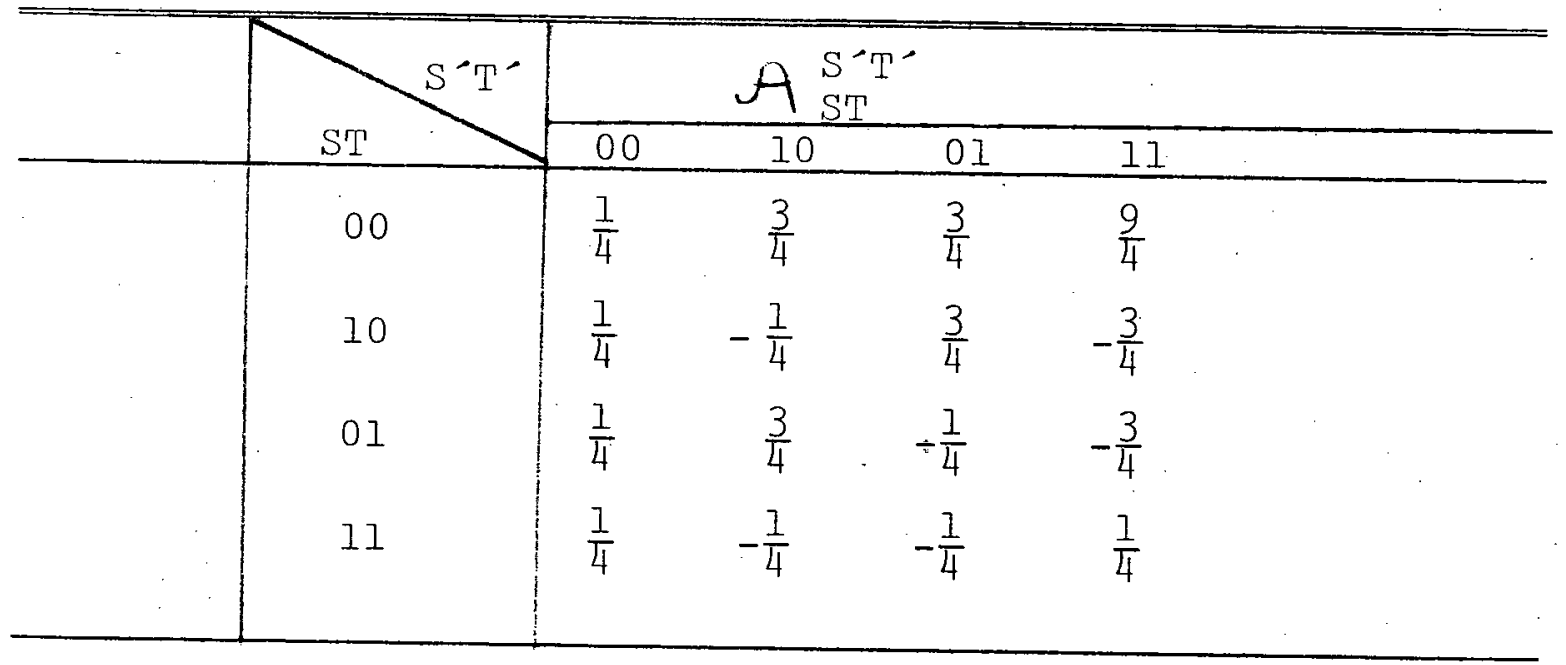


It is not obvious from the above table, but the relationship between $t^{E}(0,1)$ and $t(0,1)$ can be stated very simply. To see this note the following alternative expansion for $t(0,1)$.

$$
t(0,1)=\sum_{\mathrm{TS}} \mathrm{V}_{\mathrm{TS}}\left(\mathrm{r}_{01}\right) \mathrm{P}_{\mathrm{TS}}
$$

Here $\mathrm{P}_{\mathrm{TS}}=\mathrm{P}_{\mathrm{T}} \mathrm{P}_{\mathrm{S}}$ with $\mathrm{P}_{\mathrm{T}}$ and $\mathrm{P}_{\mathrm{S}}$ representing the usual $i-s p i n$ and spin projection operators $-P_{0}=\frac{I}{4}\left(I-\bar{\sigma}_{0} \cdot \bar{\sigma}_{1}\right)$ and $P_{1}=\frac{1}{4}\left(3+\bar{\sigma}_{0} \cdot \bar{\sigma}_{1}\right)$ for the case of ordinary spin. Unlike the previous relations for $t(0,1)$ and $t^{E}(0,1)$, where the subscripts $S$ and $T$ referred to the unit of spin and $i-s p i n$ which could be transferred from the projectile to the target nucleon through the corresponding part of the interaction, the subscripts on $\mathrm{V}_{\mathrm{TS}}\left(\mathrm{r}_{\mathrm{Ol}}\right)$ indicate that it is the component of the interaction which acts when the projectile and target nucleons are coupled to total spin $S$ and total i-spin $T$. Commonly used is the notation $\mathrm{V}_{00}=\mathrm{V}^{\mathrm{SO}}, \mathrm{V}_{10}=\mathrm{V}^{\mathrm{SE}}, \mathrm{V}_{01}=\mathrm{V}^{\mathrm{TE}}, \mathrm{V}_{11}=\mathrm{V}^{\mathrm{TO}}$ where $S O, S E$, TE, and TO refer to singlet odd, singlet even, triplet even, and triplet odd components of the interaction, respectively.

Expanding Eq. (25) and regrouping terms as in Eq. (18) gives the following relations between $t_{\mathrm{ST}}\left(\mathrm{r}_{01}\right)$ and $\mathrm{V}_{\mathrm{TS}}\left(\mathrm{r}_{01}\right)$.

$$
\begin{aligned}
& t_{00}=\frac{1}{16}\left(\mathrm{v}_{00}+3 \mathrm{~V}_{01}+3 \mathrm{~V}_{10}+9 \mathrm{~V}_{11}\right) \\
& t_{10}=\frac{1}{16}\left(-\mathrm{v}_{00}+\mathrm{v}_{01}-3 \mathrm{~V}_{10}+3 \mathrm{~V}_{11}\right) \\
& t_{01}=\frac{1}{16}\left(-\mathrm{V}_{00}-3 \mathrm{~V}_{01}+\mathrm{V}_{10}+3 \mathrm{~V}_{11}\right) \\
& t_{11}=\frac{1}{16}\left(\mathrm{~V}_{00}-\mathrm{v}_{01}-\mathrm{v}_{10}+\mathrm{v}_{11}\right)
\end{aligned}
$$


Similarly using Eq. (23) it follows that:

$$
\begin{aligned}
& t_{00}^{\mathrm{E}}=\frac{1}{16}\left(\mathrm{~V}_{00}-3 \mathrm{~V}_{01}-3 \mathrm{~V}_{10}+9 \mathrm{~V}_{11}\right) \\
& t_{10}^{\mathrm{E}}=\frac{1}{16}\left(-\mathrm{V}_{00}-\mathrm{V}_{01}+3 \mathrm{~V}_{10}+3 \mathrm{~V}_{11}\right) \\
& t_{01}^{\mathrm{E}}=\frac{1}{16}\left(-\mathrm{V}_{00}+3 \mathrm{~V}_{01}-\mathrm{V}_{10}+3 \mathrm{~V}_{11}\right) \\
& t_{11}^{\mathrm{E}}=\frac{1}{16}\left(\mathrm{~V}_{00}+\mathrm{V}_{01}+\mathrm{V}_{10}+\mathrm{V}_{11}\right)
\end{aligned}
$$

The right hand sides of Eq. (26) and Eq. (27) differ only by the signs of the even state terms. For the case of an even state force $t^{E}(0,1)=-t(0,1)$ and for an odd state force $t^{E}(0,1)=t(0,1)$. Remembering that the transition amplitude is proportional to the difference between the direct and exchange components, it is clear (insofar as the integration over internal coordinates is concerned) that the exchange amplitude contributes constructively to the direct amplitude for the even components of the interaction and destructively for the odd components. This result is a direct consequence of the fact that the internal wave function of the two nucleons is symmetric for odd states and antisymmetric for even states. It could have been seen more easily by coupling the internal coordinates of the projectile and target nucleons to good spin and i-spin before integrating in Eq. (19) and Eq. (20). This was not done, however, since Eq. (19) and Eq. (22) have the form that is needed for the remainder of this disucsion. It is interesting to note that because of antisymmetrization, "spin-flip" and "i-spin-flip" through direct inter- 
action is allowed even if it is strictly forbidden by the form of the interaction. To see this note that a Wigner force $t(0,1)=t_{00}\left(r_{01}\right)$ leads to $t^{E}(0,1)=\frac{1}{4}\left[t_{00}\left(r_{01}\right)+t_{10}\left(r_{01}\right) x\right.$ $\left.\bar{\sigma}_{0} \cdot \bar{\sigma}_{1}+t_{0 I}\left(r_{0 I}\right) \bar{\tau}_{0} \cdot \bar{\tau}_{1}+t_{I I}\left(r_{0 I}\right) \bar{\sigma}_{0} \cdot \bar{\sigma}_{1} \bar{\tau}_{0} \cdot \bar{\tau}_{I}\right]$. Such consequences appear formally because of the introduction of the pseudointeraction $t^{E}(0,1)$ into the exchange amplitude, but it should be remembered that this is simply a convenient way of cataloging the manner in which the incident projectile can be captured by the target with expulsion of a target nucleon into the final projectile state. To conclude this section note that the partial matrix element Eq. (5) can now be written as

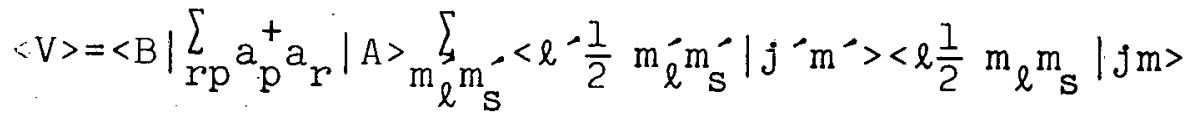

$$
\begin{aligned}
& \mathrm{m}_{\ell} \mathrm{ll}_{\mathrm{s}} \\
& x\left\{\delta\left(\bar{r}_{0}-\bar{r}_{1}\right) \int \phi_{\ell}^{m_{\ell}^{\prime}}{ }^{*}\left(\bar{r}_{2}\right)<b ; p|t(0,2)| a, r>\phi_{\ell}{ }^{l}\left(\bar{r}_{2}\right) d^{3} r_{2}-\right. \\
& \left.\Phi_{\ell}^{m_{\ell}^{-*}}\left(\bar{r}_{I}\right)<b ; p\left|t^{E}(0,1)\right| a, r>\phi_{\ell}^{m_{\ell}}\left(\bar{r}_{0}\right)\right\} \text {. }
\end{aligned}
$$

4. Final Reduction of Partial Matrix Element

Since the components of $\langle\mathrm{V}\rangle$ which correspond to the tiansfer of total angular momentum $J$ are of interest it is convenient to couple the creation and annihilation operator in Eq. (28) to good J.

$A_{J M}\left(j^{\prime} \tau^{\prime} ; j \tau\right)=\sum_{m m^{\prime}},<j^{\prime} j m^{\prime} ;-m \mid J M_{J}>(-1)^{j-m} a_{j^{\prime} m^{\prime} \tau} \tau^{-a_{j m}}$ 
The phase factor $(-1)^{j-m}$ insures that $A_{J M_{J}}$ has the correct transformation properties under rotation. If $i-s p i n$ is considered to be a good quantum number additional coupling to good $\mathrm{T}$ is necessary.

$A_{J M_{J}}^{\mathrm{TM}_{\mathrm{T}}}\left(j^{\prime} j\right)=\frac{\sum}{\tau \tau},<\frac{1}{2} \frac{1}{2} \tau^{\prime},-\tau \mid \mathrm{TM}_{\mathrm{T}}>(-1)^{1 / 2-\tau} \mathrm{A}_{J M_{J}}\left(j^{\prime} \tau^{\prime} ; j \tau\right)$

For these two cases it immediately follows that

$$
\begin{aligned}
& \left\langle B\left|\sum_{p r} a_{p^{+}}^{+} a_{r}\right| A\right\rangle=\sum_{\substack{j m^{\prime} \tau^{\prime} \tau^{\prime} \\
J M_{j}}}(-1)^{j-m^{\prime}}\left\langle j^{\prime} j m^{\prime},-m \mid J M_{J}\right\rangle\left\langle J_{A} J M_{A} M_{J} \mid J_{B} M_{B}\right\rangle \\
& x<J_{B}|| A_{J}\left(j{ }^{-} \tau^{\prime} ; j \tau\right)|| J_{A}>
\end{aligned}
$$

and

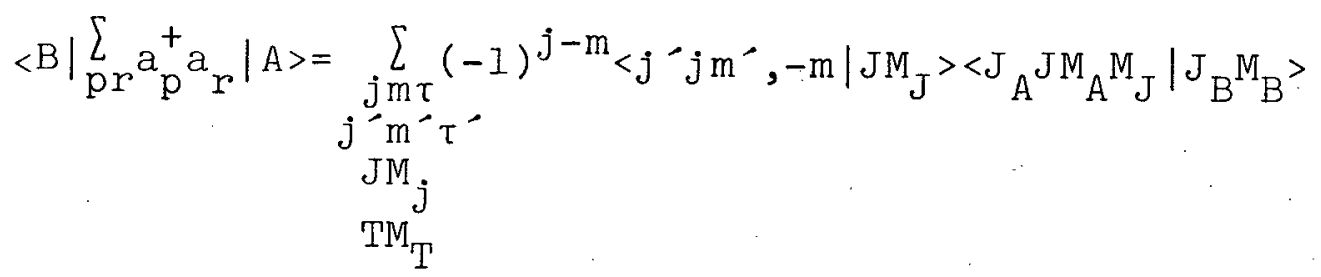

$$
\begin{aligned}
& \mathrm{x}(-1)^{1 / 2-\tau}<\frac{1}{2} \frac{1}{2} \tau^{-},-\tau\left|\mathrm{TM}_{\mathrm{T}}><\mathrm{T}_{\mathrm{A}} \mathrm{TM}_{\mathrm{T}_{\mathrm{A}}} \mathrm{M}_{\mathrm{T}}\right| \mathrm{T}_{\mathrm{B}} \mathrm{M}_{\mathrm{T}_{\mathrm{B}}}> \\
& x<J_{B} T_{B}|| A_{J}^{T}(j-j)|| J_{A} T_{A}>.
\end{aligned}
$$

Eq. (31) or Eq. (32) and the results of Eq. (19) and Eq. (22) are inserted into Eq. (28) and a recoupling operation is performed to introduce the transferred orbital angular momentum. The necessary identity is 
$\sum_{m_{S} m_{S}^{-}}(-1)^{j-m^{\prime}}\left\langle j^{\prime} j m^{\prime},-m\left|J M_{J}><\frac{1}{2} S m_{S} \lambda\right| \frac{1}{2} m_{S}^{-}><\ell \frac{1}{2} m_{\ell} m_{S}\right| j m>$ $x<\ell-\frac{1}{2} m_{\ell^{-} m_{S}^{-}} \mid j^{\prime} m^{\prime}>=\sum_{L M} \frac{\sqrt{2} \hat{j}-\hat{j} \hat{L}^{2}}{\hat{\ell}^{-}}(-1)^{L-\ell+\ell^{\prime}}\langle\operatorname{LSM} \lambda| J M_{J^{\prime}}>$ $x<L \ell M_{\ell} \mid \ell^{\prime} m_{\ell}>\underline{\bar{x}}\left(j-j J ; \ell^{\prime} \ell L ; \frac{1}{2} \frac{1}{2} S\right)$.

Rearranging some Clebsch-Gordan coefficients, summing over indices if necessary, and comparing with Eq. (17) allows the identification

$\sum_{L_{a} M_{a}} Y_{L_{b} M_{b}}^{*}\left(\hat{r}_{0}\right) Y_{L_{a} M_{a}}^{*}\left(\hat{r}_{1}\right) F_{L L_{b} L_{a}}^{S J}\left(r_{0}, r_{1}\right)<L_{b} L_{a} M_{b} M_{a}|L M\rangle=$ $\mathrm{L}_{\mathrm{b}} \mathrm{M}_{\mathrm{b}}$

$$
\begin{aligned}
& \sum_{j j^{-i}} L_{2} \hat{j}^{j} \hat{j}^{-} \hat{L}^{2} \hat{\ell}^{-1} \hat{S} \underline{X}\left(j^{\prime} j J ; \ell^{\prime} \ell L ; \frac{1}{2} \frac{1}{2} S\right) \sum_{T} B(J T)_{m_{\ell} m_{\ell}^{-}}^{\sum}\left\langle L \ell M m_{\ell} \mid \ell^{\prime} m_{\ell}^{\prime}\right\rangle \\
& x(-1)^{L-\ell+\ell^{\prime}}\left\{\delta\left(\bar{r}_{0}-\bar{r}_{1}\right) \int \phi_{\ell^{\ell^{\prime}}}^{m^{*}}\left(\bar{r}_{2}\right) t_{S T}\left(r_{02}\right) \phi_{\ell}^{m_{l}}\left(\bar{r}_{2}\right) d^{3} r_{2}-\right. \\
& \left.\phi_{\ell}^{\mathrm{m}_{\ell}^{*}}\left(\bar{r}_{1}\right) t_{\mathrm{ST}}^{\mathrm{E}}\left(\mathrm{r}_{01}\right) \phi_{\ell}^{\mathrm{m}_{\ell}}\left(\bar{r}_{0}\right)\right\} \text {. }
\end{aligned}
$$

In these relations $\underline{\bar{x}}$ (abc;def;ghi) is a $9-j$ symbol $1^{58}$ and

$$
\begin{aligned}
& \left.\mathrm{B}(\mathrm{JT})=\sqrt{2} \hat{\mathrm{T}}<\mathrm{T}_{\mathrm{A}} \mathrm{TM}_{\mathrm{T}_{\mathrm{A}}}, \mathrm{M}_{\mathrm{T}_{\mathrm{B}}}-\mathrm{M}_{\mathrm{T}_{\mathrm{A}}}\left|\mathrm{T}_{\mathrm{B}} \mathrm{M}_{\mathrm{T}_{\mathrm{B}}}><\frac{1}{2} \mathrm{~T} \tau_{\mathrm{b}}, \tau_{a}-\tau_{\mathrm{b}}\right| \frac{1}{2} \tau_{a}\right\rangle \\
& x<\mathrm{J}_{B} \mathrm{~T}_{\mathrm{B}}\left\|\mathrm{A}_{\mathrm{J}}^{\mathrm{T}}\left(j^{\prime} j\right)\right\| \mathrm{J}_{\mathrm{A}^{\mathrm{T}} \mathrm{T}}>
\end{aligned}
$$

for the case of good i-spin or

$$
B(J T)=\sum_{\tau \tau}, \hat{T}^{2}<\frac{1}{2} T \tau, \tau a^{-\tau} \tau_{b}\left|\frac{1}{2} \tau^{\prime}><\frac{1}{2} T \tau_{b} ; \tau_{a}-\tau_{b}\right| \frac{1}{2} \tau_{a}>\left\langle J_{B}|| A_{J^{\prime}}\left(j^{\prime} \tau^{\prime} ; j \tau\right)|| J_{A}\right\rangle
$$


when i-spin is not considered to be a good quantum number for the target.

The meaning of the reduced matrix elements appearing in Eq. (35) and Eq. (36) can be illustrated by writing them in a somewhat more familiar form. By inverting the wignerEckart Theorem the reduced matrix element appearing in Eq. (36) can be written as follows.

$$
\begin{aligned}
<\alpha_{B} J_{B}|| A_{J}\left(j^{\prime} \tau^{\prime} ; j \tau\right)|| \alpha_{A} J_{A}>= & \sum_{M_{A} M_{J}}<J_{A} J M_{A} M_{J} \mid J_{B} M_{B}>(-1)^{j-m} \\
& x<j^{\prime} j m^{\prime},-m \mid J M_{J^{\prime}}> \\
& x<\alpha_{B} J_{B} M_{B}\left|a_{j}^{+} m^{\prime} \tau^{-a} a_{j m}\right| \alpha_{A} J_{A} M_{A}>
\end{aligned}
$$

The Greek letters have been re-introduced to allow complete specification of the nuclear states. Since $A$ and $B$ are antisymmetric states containing $n$ nucleons it follows that

$$
\begin{aligned}
& <\alpha_{B} J_{B} M_{B}\left|a_{j}^{+} m^{\prime} \tau^{-a_{j m \tau}}\right| \alpha_{A} J_{A}^{M_{A}}>=\alpha_{p^{\prime} p_{p} M_{p}}<\alpha_{B} J_{B} M_{B}\left|a_{j^{\prime} m^{\prime}}^{+} \tau^{-}\right| \alpha_{p} J_{p} M_{p}> \\
& x<\alpha_{p} J_{p} M_{p}\left|a_{j m \tau}\right| \alpha_{A} J_{A} M_{A}>
\end{aligned}
$$

where the complete set of antisymmetric states composed of $n-1$ nucleons has been introduced. The reduced matrix elements in Eq. (35) and Eq. (36) are simply related to the coefficients of fractional parentage (c.f.p.) 59 and all results can be put in the form of the usual fractional parentage expansion. 58 The definitive relation is 
$\left.<\alpha_{p} J_{p} M_{p}\left|a_{j m \tau}\right| \alpha_{A} J_{A} M_{A}>=n n^{I / 2}<\alpha_{p} J_{p} ; j \tau \mid\right\} \alpha_{A} J_{A}><J_{p} j M_{p} m \mid J_{A} M_{A}>$.

Using results (38) and (39) In Eq. (37) and summing over projections gives:

$$
\begin{aligned}
&<\alpha_{B} J_{B} \| A_{J}\left(j^{\prime} \tau^{\prime} ; j \tau\right)|| \alpha_{A} J_{A}>=S\left(J_{A} J_{B} J ; j^{\prime} \tau \tau^{\prime}\right) \hat{J} \hat{j}^{--1} \\
& S\left(J_{A} J_{B} J ; j^{\prime} \tau \tau^{\prime}\right)=\left.\left.\alpha_{p} \sum_{p} n<\alpha_{p} J_{p} ; j \tau \mid\right\} \alpha_{A} J_{A}><\alpha_{p} J_{p} ; j^{\prime} \tau-\mid\right\} \alpha_{B} J_{B}> \\
& x\left\{\hat{J}_{A} \hat{j}^{-} W\left(j^{\prime} j^{\prime} J_{A} J_{B} ; J J_{p}\right)(-1) J_{p}-J_{A}+J^{-} j^{-}\right\} .
\end{aligned}
$$

For the case of good i-spin it follows that:

$$
\begin{aligned}
& \left.<\alpha_{B} J_{B} T_{B} \| A_{J}^{T}(j-j)|| \alpha_{A} J_{A} T_{B}\right\rangle=S\left(J_{A} J_{B} J ; T_{A} T_{B} T ; j j^{\prime}\right) \hat{J} \hat{j}^{--1} \hat{T}(2)^{-1 / 2} \\
& \left.S\left(J_{A} J_{B} J^{\prime} ; T_{A} T_{B} T ; j j^{\prime}\right)={\underset{p}{\alpha} J_{p} T_{p}}_{\sum_{p}} n<\alpha_{p} J_{p} T_{p} ; j \mid\right\} \alpha_{A} J_{A} T_{A}> \\
& \left.x<\alpha_{p} J_{p}^{T} T^{T} ;-\mid\right\} \alpha_{B} J_{B} T_{B}> \\
& x\left\{\hat{J}_{A} \hat{j}^{-} W\left(j j^{\prime} J_{A} J_{B} ; J J_{p}\right)(-I)^{J_{p}-J_{A}+J-J^{\prime}}\right\} \\
& x\left\{\hat{T}_{A} \sqrt{2} W\left(\frac{1}{2} \frac{1}{2} T_{A} T_{B} ; T_{p}\right)(-1)^{T_{p}-T_{A}+T-1 / 2}\right\} \text {. }
\end{aligned}
$$

'I'he spectroscopic amplitudes $S$ which have been introduced are simply partial sums of the complete fractional parentage expansion of the partial matrix element. They contain the weighting imposed by the nuclear structure for the contribution to the transition due to a single nucleon going from the initial state $j(j \tau)$ to the final state $j^{-}\left(j^{-} \tau^{\prime}\right)$. The factors 
appearing in Eq. (40) and (41) which have not been included in $S$ guarantee a convenient interpretation of the remaining factors in Eq. (34).

When $i-s p i n$ is not considered to be a good quantum number it is useful to redefine the interaction by performing the sum over T when Eq. (36) is used in Eq. (34), i.e. define

$t_{S \tau_{a} \tau_{b} \tau \tau^{-}}\left(r_{0 I}\right)=\sum_{T^{2}}^{\hat{T}^{2}}<\frac{1}{2} T \tau, \tau_{a}-\tau_{b}\left|\frac{1}{2} \tau^{-}><\frac{1}{2} T \tau_{b}, \tau_{a}-\tau_{b}\right| \frac{1}{2} \tau_{a}>t_{S T}\left(r_{0 I}\right)$

with a corresponding relation for $t^{\mathrm{E}}\left(r_{01}\right)$. Table 2 gives the coefficients in the above expansion for the various combinations of $i-s p i n$ projections. The first entry in each column is the coefficient for $T=0$ while the second is for $T=1$. I-spin projection equal to $\frac{1}{2}$ denotes a proton and $-\frac{1}{2}$ denotes a neutron. Incompatible projection combinations are indicated by a dash. The table simply shows that for inelastic proton or neutron scattering the proton-proton and neutron-neutron interaction is $t_{S O}{ }^{+t} t_{S I}$ while the neutron-proton interaction is $t_{S O}{ }^{-t} \mathrm{SI}$. Further it illustrates that only the iso-vector part of the interaction contributes to the charge exchange reactions. Since it will always be clear what reaction is being considered no ambiguity should result if the subscripts $\tau_{a} \tau_{b}$ are dropped from $t$ in $E q$. (42). For the $\left(p, p^{\prime}\right)$ reaction it is also convenient to use the subscripts pp and pn corresponding to $\tau_{a}=\tau=\frac{1}{2}$ and $\tau_{a}=-\tau=\frac{1}{2}$, respectively. 
TABLE 2.--Interaction components when 1 -spin is not used.

\begin{tabular}{l|c|cccc}
\hline REACTION & $\tau_{a \tau_{b}} \tau^{\prime}$ & $\frac{1}{2} \frac{1}{2}$ & $-\frac{1}{2}-\frac{1}{2}$ & $\frac{1}{2}-\frac{1}{2}$ & $-\frac{1}{2} \frac{1}{2}$ \\
\hline$\left(p, p^{\prime}\right)$ & $\frac{1}{2} \frac{1}{2}$ & 1,1 & $1,-1$ & - & - \\
$\left(n, n^{\prime}\right)$ & $-\frac{1}{2}-\frac{1}{2}$ & $1,-1$ & 1,1 & - & - \\
$(p, n)$ & $\frac{1}{2}-\frac{1}{2}$ & - & - & - & 0,2 \\
$(n, p)$ & $-\frac{1}{2} \frac{1}{2}$ & - & - & 0,2 & - \\
\hline
\end{tabular}

Now Eq. (40)-(42) are incorporated into Eq. (34). In addition $t\left(r_{01}\right), t^{E}\left(r_{01}\right)$, and $\delta\left(\bar{r}_{0}-\bar{r}_{1}\right)$ are expanded in spherical harmonics. This expansion is defined by

$$
f\left(r_{0 I}\right)=\sum_{L M} f_{L}\left(r_{0} ; r_{I}\right) Y_{L M}^{*}\left(\hat{r}_{0}\right) Y_{L M}\left(\hat{r}_{I}\right)
$$

with

$$
f_{L}\left(r_{0} ; r_{I}\right)=2 \pi \int_{-1}^{1} \cdot P_{L}(\cos \alpha) f\left(r_{01}\right) d \cos \alpha
$$

where $\alpha$ is the angle between $\bar{r}_{0}$ and $\bar{r}_{1}$. The single particle

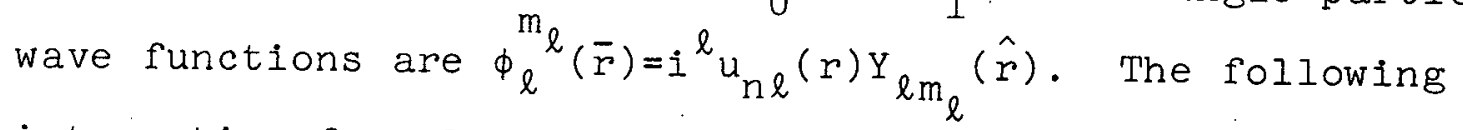
integration formula facilitates the inversion of the resulting expression.

$$
\begin{aligned}
\int Y_{L_{1} M_{1}}(\hat{r}) Y_{L_{2} M_{2}}(\hat{r}) Y_{L_{3} M_{3}}(\hat{r}) d \Omega= & (4 \pi)^{-I / 2} \hat{L}_{1} \hat{L}_{2} \hat{L}_{3}^{-1}<L_{1} L_{2} 00 \mid L_{3} 0> \\
& x<L_{1} L_{2} M_{1} M_{2} \mid L_{3},-M_{3}>(-1)^{M}
\end{aligned}
$$


Contraction of Clebsch-Gordan coefficients and recoupling in the exchange component, as was done previously in integrating over the internal coordinates (see Eq. (2l)), gives for the case of good $i-s p i n$

$$
\begin{aligned}
& \mathrm{F}_{\mathrm{LL}_{\mathrm{b}} \mathrm{L}_{\mathrm{a}}}^{\mathrm{SJ}}\left(\mathrm{r}_{0}, \mathrm{r}_{\mathrm{I}}\right)= \\
& \left.\sum_{\mathrm{T}}^{j}-\sqrt{2} \hat{\mathrm{T}}<\mathrm{T}_{\mathrm{A}} \mathrm{TM}_{\mathrm{T}_{\mathrm{A}}}, \mathrm{M}_{\mathrm{T}_{\mathrm{B}}}-\mathrm{M}_{\mathrm{T}_{\mathrm{A}}}\left|\mathrm{T}_{\mathrm{B}} \mathrm{M}_{\mathrm{T}_{\mathrm{B}}}><\frac{1}{2} \mathrm{~T} \tau_{\mathrm{b}}, \tau_{a}-\tau_{\mathrm{b}}\right| \frac{1}{2} \tau \mathrm{a}\right\rangle \\
& x_{i}^{L-\ell^{\prime}-\ell} \sqrt{2} \hat{j} \ell \hat{\ell} \hat{L} \hat{L} \hat{S} \hat{J T} \underline{\underline{X}}\left(j j^{\prime} j J ; \ell^{\prime} \ell L ; \frac{1}{2} \frac{1}{2} S\right) S\left(J_{A} J_{B} J ; T_{A} T_{B} T ; j j^{\prime}\right) \\
& x(4 \pi)^{-1}\left\{\hat{L}_{a} \hat{L}_{b} \hat{L}^{-2} I_{S T L}^{\ell^{\prime} \ell}\left(r_{0}\right)<\ell \ell^{-} 00\left|I 0><L_{a} L_{b} 00\right| L 0>\frac{\delta\left(r_{0}-r_{I}\right)}{r_{0}^{2}}\right. \\
& -u_{n}^{*} \ell^{-}-\left(r_{1}\right) u_{n \ell}\left(r_{0}\right) \sum_{L}-(-1)^{L^{-}} \hat{L}^{-2} W\left(\ell L_{b} \ell^{\prime} L_{a} ; L^{\prime} L\right)\left\langle\ell L^{\prime} 00 \mid L_{b} 0\right\rangle \\
& \left.x<\ell-L^{-} 00 \mid L_{a} 0>t_{S T L}^{E}\left(r_{0} ; r_{I}\right)\right\}
\end{aligned}
$$

For the case $i-s p i n$ is ignored,

$$
\begin{aligned}
& F_{L L_{b} L_{a}}^{S J}\left(r_{0}, r_{I}\right)=
\end{aligned}
$$

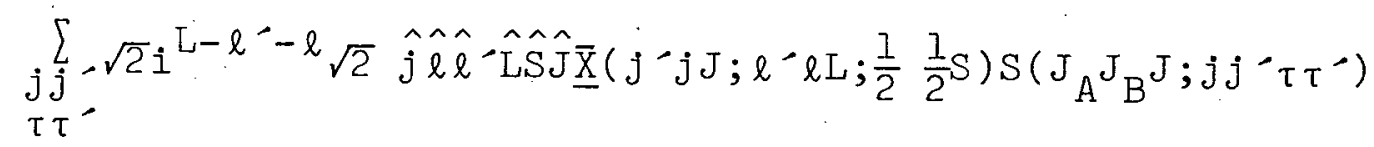

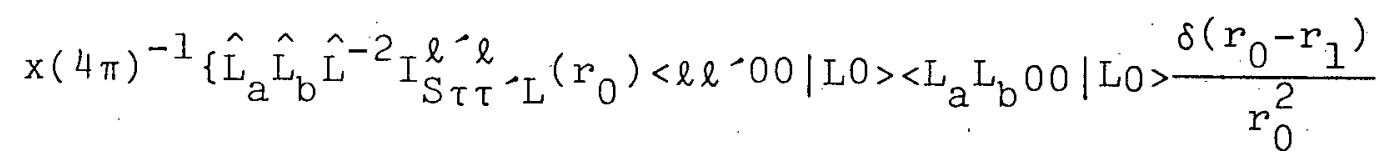

$$
\begin{aligned}
& -u_{n}^{*} \ell^{\prime}-\left(r_{I}\right) u_{n \ell}\left(r_{0}\right) \sum_{L}-(-I)^{L-} \hat{L}^{-2} W\left(\ell L_{b} \ell^{-} L_{a} ; L-L\right)<\ell L-00 \mid L_{b} 0> \\
& \left.x<\ell^{\prime} L^{\prime} 00 \mid L_{a} 0>t_{S \tau \tau}^{E} \tau^{-}-\left(r_{0} ; r_{1}\right)\right\} \text {. }
\end{aligned}
$$


In these expressions

$I_{S^{\prime} \ell^{\prime}}^{\ell^{\prime}}=\int u_{n^{\prime} \ell}^{*}-\left(r_{2}\right) t_{S T L}\left(r_{0} ; r_{2}\right) u_{n \ell}\left(r_{2}\right) r_{2}^{2} d r_{2}$

and

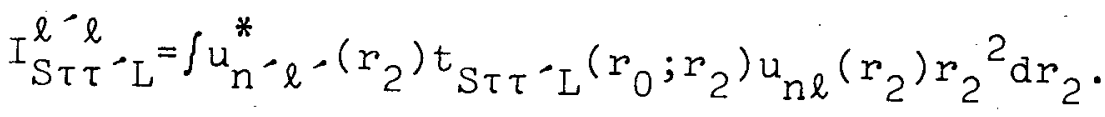

Using the symmetry properties of the Clebsch-Gordan coefficients it is easy to see that the first term in Eq. (46) and $\mathrm{Eq}$. (47) has the form indicated in Eq. (15). Identification of $\tilde{D}^{L S J}\left(r_{0}\right)$ follows directly.

$\tilde{D}^{L S J}\left(r_{0}\right)=\sum_{j}^{j} \sum_{T}^{j}-\sqrt{2} \hat{T}<T_{A} T_{T_{A}}, M_{T_{B}}-M_{T_{A}}\left|T_{B} M_{T_{B}}><\frac{1}{2} T \tau_{b}, \tau_{a}-\tau_{b}\right| \frac{1}{2} \tau_{2}>$

$$
\begin{aligned}
& x S\left(J_{A} J_{B} J ; T_{A} T_{B} T ; j j^{\prime}\right) \\
& x \int\left\langle j-\frac{1}{2}|| \frac{\delta\left(r_{1}-r_{2}\right)}{r_{2}^{2}} T^{L S J}(2) \tau^{T}(2)|| j \frac{1}{2}>t_{S T L}\left(r_{0} ; r_{1}\right) r_{1}^{2} d r_{1}\left(46^{\prime \prime}\right)\right.
\end{aligned}
$$$$
\tilde{D} L S J\left(r_{0}\right)=\sum_{\substack{j j^{\prime} \\ \tau}} \sqrt{2} S\left(J_{A} J_{B} J ; j j^{\prime} \tau \tau^{\prime}\right)
$$$$
x \int<j-|| \frac{\delta\left(r_{1}-r_{2}\right)}{r_{2}^{2}} T^{L S J}(2) \| j>t_{S \tau \tau \sim L}\left(r_{0} ; r_{1}\right) r_{1}^{2} d r_{1}
$$

The spin-angle tensor has been introduced, i.e.

$$
\mathrm{T}_{\mathrm{M}_{J}}^{\mathrm{LSJ}}=\sum_{\mathbb{M} \lambda}<\operatorname{LSM} \lambda \mid J M_{J}>i \mathrm{Y}_{\mathrm{LM}} \sigma_{\lambda}^{S}
$$

and the reduced matrix elements appearing in these relations are given by 


$$
\begin{aligned}
& <j-\frac{1}{2}|| \frac{\delta\left(r_{1}-r_{2}\right)}{r_{2}^{2}} T^{L S J}(2) \tau^{T}(2)|| j \frac{1}{2}>= \\
& i^{L+\ell-\ell^{-}}(4 \pi)^{-1 / 2} \sqrt{2} \hat{j} \hat{\ell} \hat{L S} \hat{J} \hat{T}<L \ell 00 \mid \ell^{-} 0>\underline{\bar{X}}\left(j-j J ; \ell^{\prime} \ell L ; \frac{1}{2} \frac{1}{2} S\right) \\
& \quad x u_{n}^{*} \ell^{-}-\left(r_{I}\right) u_{n \ell}\left(r_{I}\right)
\end{aligned}
$$

and

$$
\begin{aligned}
& <j^{\prime}\left\|\frac{\delta\left(r_{1}-r_{2}\right)}{r_{2}^{2}} T^{L S J}(2)\right\| j>= \\
& i^{L+\ell-l^{\prime}}(4 \pi)^{-1 / 2} \sqrt{2} \hat{j} \hat{\ell} \hat{L S} \hat{J}<L \ell 00 \mid \ell^{\prime} 0>\underline{\underline{X}}\left(j^{\prime} j J ; \ell^{\prime} \ell L ; \frac{1}{2} \frac{1}{2} S\right) \\
& x u_{n^{\prime} \ell^{-}}^{*}\left(r_{I}\right) u_{n}\left(r_{I}\right) .
\end{aligned}
$$

The $\sqrt{2}$ and i-spin factors in Eq. (46") and Eq. (47") appear because the partial matrix element contains the integrations over internal projectile coordinates.

Examination of the above relations shows that the direct component of the partial matrix element for a given $L$ depends on only one multipole coefficient of the interaction while the exchange component depends on several of these coefficients. In addition one of the more interesting consequences of antisymmetrization is noted. This concerns the relation between the L-transfer and parity change $(\Delta \pi)$ in a transition. In inelastic nucleon-nucleus scattering no change in the intrinsic parity of the projectile is involved, thus any change in the parity of the target during a transition requires a corresponding change in parity in the state of relative projectile-target 
motion. This condition is displayed in Eq. (46) and (47) where it is obvious that $\Delta \pi=(-1)^{L_{a}+L_{b}}=(-1)^{l+l^{\prime}}$. These same relations illustrate that the direct component of the transition amplitude vanishes unless $\Delta \pi=(-1)^{L}$. Such a relation does not exist for the exchange component and there will be contributions to the cross sections when $\Delta \pi \neq(-1)^{\mathrm{L}}$. In the local D.W.A. one has the selection rules indicated in Eq. (7) along with those given in Eq. (8) for i-spin and the additional relation between L-transfer and $\Delta \pi$. For a given value of $J,(L S J)$ can take the values $(J, 0, J)$ and $(J, I, J)$ or $(J-I, I, J)$ and $(J+l, I, J)$. With the inclusion of the exchange component all four triads are allowed for a given $J$. The contributions to the cross section with $(-1)^{L} \neq \Delta \pi$ are referred to as non-normal transfers.

5. Zero-Range Interaction

A special case of some interest is an interaction of zero-range, i.e. $t\left(r_{01}\right)=T\left(\bar{r}_{0}-\bar{r}_{1}\right)$ which leads to $t\left(r_{0} ; r_{1}\right)=$ $\mathrm{T} \frac{\delta\left(r_{0}-r_{1}\right)}{r_{0}^{2}}$ which does not depend on $L$; therefore the multipole coefficient of the exchange interaction can be factored out of the sum over $\mathrm{L}^{-}$in $\mathrm{Eq} .(46)$ and (47). The sum then yields

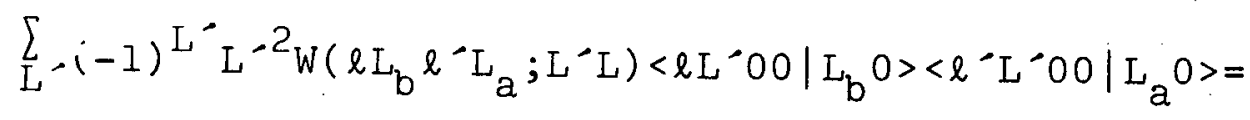

$$
\begin{aligned}
& \hat{L}_{a} \hat{L}_{b} \hat{L}^{-2}<\ell l-00\left|L 0><L_{a} L_{b} 00\right| L 0>
\end{aligned}
$$

which gives the exchange component of $\mathrm{F}_{\mathrm{LI}_{\mathrm{b}} \mathrm{L}_{\mathrm{a}}}^{\mathrm{SJ}}\left(\mathrm{r}_{0} ; \mathrm{r}_{1}\right)$ the same form as the direct component. The following expressions are 
obtained for $\tilde{F}^{\mathrm{LSJ}}\left(\mathrm{r}_{0}\right)$ for the case of good $i-s p i n$ and the case when $i-s p i n$ is ignored, respectively.

$$
\begin{aligned}
\tilde{F^{L S J}}\left(r_{0}\right)= & \underset{j}{j_{T}^{j}}-\sqrt{2} \hat{T}<T_{A} T_{T_{A}}, M_{T_{B}}\left|T_{B} M_{T_{B}}><\frac{1}{2} T \tau_{b}, \tau_{a}-\tau_{b}\right| \frac{1}{2} \tau_{a}> \\
& x S\left(J_{A} J_{B} J ; T_{A} T_{B} T ; j j^{-}\right)\left(T_{S T}-T_{S T}^{E}\right) \\
& x<j-\frac{1}{2}|| \frac{\delta\left(r_{0}-r_{2}\right)}{r_{0}^{2}} T^{L S J}(2) \tau^{T}(2)|| j \frac{1}{2}>
\end{aligned}
$$

or

$$
\begin{gathered}
\tilde{F}^{L S J}\left(r_{0}\right)=\sum_{\substack{j j^{\prime} \\
\tau^{\prime}}}-\sqrt{2} S\left(J_{A} J_{B^{\prime}} j ; j j^{\prime} \tau \tau^{\prime}\right)\left(T_{S \tau \tau^{\prime} L}-T_{S \tau \tau^{\prime} L^{\prime}}^{E}\right) \\
\quad x<j-\left\|\frac{\delta\left(r_{0}-r_{2}\right)}{r_{0}^{2}} T^{L S J}(2)\right\| j>.
\end{gathered}
$$

It is clear that the effect of antisymmetrization in the limit of zero range is simply to renormalize the strength of the interaction. Further, non-normal transfers are not allowed in. this limit.

6. Approximate Treatment of Antisymmetrization

The approximations used to treat antisymmetrization in this work are based on the fact the exchange scattering, as compared to the direct scattering, is sensitive to a particular momentum component of the two-body interaction. To see this note the form which the basic integrals of the D.W.A. transition amplitude, Eq. (I), have in a momentum representation. 


$$
\begin{aligned}
& I_{\mathrm{dir}}=(2 \pi)^{-9} \\
& x \int x_{b}{ }^{(-) *}\left(\bar{k}_{1}^{\hat{\imath}}\right){ }_{p}^{*}\left(\bar{k}_{1}+\bar{k}_{2}-\bar{k}_{1}^{\hat{1}}\right) t\left(\left|\bar{k}_{1}-\bar{k}_{1}^{\hat{\imath}}\right|\right) \phi_{r}\left(\bar{k}_{2}\right) x_{a}^{(+)}\left(\bar{k}_{1}\right) d \tau \\
& I_{\text {ex }}=(2 \pi)^{-9} \\
& x \int x_{\mathrm{b}}^{(I) *}\left(\overline{\mathrm{k}}_{1}^{\hat{l}}\right) \phi_{\mathrm{p}}^{*}\left(\overline{\mathrm{k}}_{1}+\overline{\mathrm{k}}_{2}-\overline{\mathrm{k}}_{1}\right) t^{\mathrm{E}}\left(\left|\overline{\mathrm{k}}_{1}-\overline{\mathrm{k}}_{2}\right|\right) \phi_{\mathrm{r}}\left(\overline{\mathrm{k}}_{2}\right) \mathrm{x}_{\mathrm{a}}^{(+)}\left(\overline{\mathrm{k}}_{1}\right) \mathrm{d} \tau
\end{aligned}
$$

In the direct scattering the projectile goes from the initial state $\overrightarrow{\mathrm{k}}_{1}$ to the final state $\overrightarrow{\mathrm{k}}_{1}$ by transferring momentum $\overline{\mathrm{q}}=\overline{\mathrm{k}}_{1}-\overline{\mathrm{k}}_{\mathrm{l}}$ to the bound particle. In the exchange scattering the projectile is captured and transfers momentum $\bar{p}=\bar{k}_{1}-\bar{k}_{2}$ to the bound particle thereby expeling it from the target with momentum $\bar{k}_{1}$. Introducing the initial and final relative momentum $\bar{k}$ and $\bar{k}^{\prime}$ of the two nucleons it follows that:

$$
\begin{aligned}
\bar{q} & =\bar{k}-\bar{k}^{-} \\
p^{2} & =\frac{1}{2}\left(k^{2}+k^{-2}\right)-q^{2}
\end{aligned}
$$

I'o the extent that the scattering is governed by the kinematics of the nucleon-nucleus system, $i . e$. on the average the bound particle is initially at rest in the $1 \mathrm{ab}$ and for scattering at a particular angle in the nucleon-nucleus center of mass the average value of $\bar{q}$ is the assymptotic value, it follows that:

$$
\begin{aligned}
& k^{-2}=k^{2}+\frac{N-1}{2 N} q^{2} \\
& p^{2}=4 k^{2}-\frac{1}{N} q^{2} \approx k_{L A B}^{2}
\end{aligned}
$$


where $\mathrm{N}$ is the number of particles in the target and it has been assumed that no energy is lost in exciting the target. For the case of the $K-K$ and effective range forces the exchange integral is approximated by evaluating $t^{E}\left(\left|\bar{k}_{1}-\bar{k}_{2}\right|\right)$ at $k_{L A B}^{2}$ and removing it from the integral. In a coordinate representation Eq. (5I) becomes

$$
I_{e x}=t^{E}\left(k_{L A B}^{2}\right) \int x_{b}^{(-) *}\left(\bar{r}_{0}\right) \phi_{p}^{*}\left(\bar{r}_{I}\right) \delta\left(\bar{r}_{0}-\bar{r}_{I}\right) \phi_{r}\left(\bar{r}_{1}\right) x_{a}^{(+)}\left(\bar{r}_{0}\right) d^{3} r_{0} d^{3} r_{I} .
$$

The following expressions result for $\check{E}^{L S J}\left(r_{0}\right)$ :

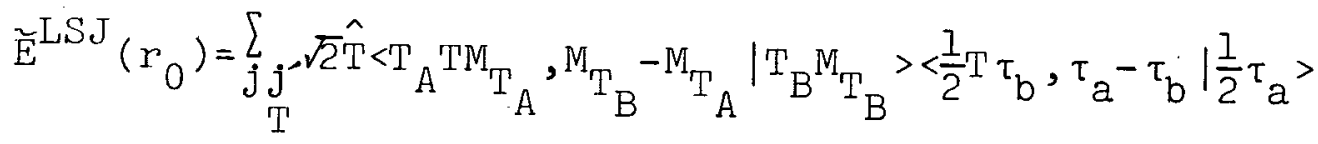

$$
\begin{aligned}
& x S\left(J_{A} J_{B} J^{\prime} ; T_{A} T_{B}^{T} ; j^{\prime}\right) A_{S T}^{(I)}\left(\lambda_{0}^{2}\right) \\
& x<j-\frac{1}{2} \| \frac{\delta\left(r_{0}-r_{2}\right)}{r_{0}^{2}} T^{L S J}(2) \tau^{T}(2)|| j \frac{1}{2}>
\end{aligned}
$$

$\widetilde{E}^{L S J}\left(r_{0}\right)=\sum_{j j^{\prime}-}, \sqrt{2} S\left(J_{A} J_{B^{\prime}} J ; j^{\prime} \tau \tau^{\prime}\right) A_{S \tau \tau}(I)-\left(\lambda_{0}^{2}\right)$

$$
x<j-\| \frac{\delta\left(r_{0}-r_{2}\right)}{r_{0}^{2}} T^{L S J}(2)|| j>
$$

$A^{(I)}\left(\lambda_{0}^{2}\right)=\left[-\int e^{-i \bar{\lambda} \cdot \bar{r}_{0 I} E} E_{t}\left(r_{0 I}\right) d^{3} r_{0 I}\right]{ }_{\lambda}^{2}=\lambda_{0}^{2}$

In Eq. (57) $\lambda_{0}^{2}=k_{L A B}^{2}=2 M E_{L A B} / \hbar^{2}$. where $M$ is the nucleon mass. This is the simplest approximation which can be made to treat the exchange component of the D.W.A. transition amplitude. Comparing the above relations for $\widetilde{E}^{L S J}\left(r_{0}\right)$ with. those for $\tilde{D}^{\mathrm{LSJ}}\left(r_{0}\right)$ in section 4 of this chapter leads to 
the following qualitative conclusions about the properties of exchange scattering as treated in this approximation.

(i) The angular distribution for exchange scattering will fall off slower in angle than that for direct scattering.

(ii) The importance of exchange scattering will increase as the energy decreases.

(iii) The importance of exchange scattering with respect to direct scattering should increase with increasing Ltransfer.

(iv) The direct and exchange amplitudes will be roughly in phase. 'lhese conclusions require assumptions regarding the behavior of the multipole coefficients and Fourier transforms of the interactions being consiaered, i.e. $A^{(1)}\left(\lambda_{0}^{2}\right)$ increases with decreasing $\lambda_{0}^{2}$ and $t_{L}\left(r_{0} ; r_{1}\right)$ falls off with increasing $L$. The assumed behaviour is typical and the qualitative observations are in agreement with the results of exact calculations.17-19 Quantitative comparisons are made in Chapter 5.

One can object to this approximation for two reasons: (i) it does not preserve the possibility of non-normal transfers and (ii) the validity of taking $t^{E}\left(\left|\bar{k}_{1}^{\prime}-\bar{k}_{2}\right|\right)$ out of integral in Eq. (5I) is strictly valid only at high energies where the importance of exchange scattering is diminished. The quantitative comparisons in Chapter 5 serve as an answer to the latter objection. Because of objection (i) it is necessary that non-normal transfers be unimportant if this approximation is to be useful. One reason for favoring normal transfers over non-normal transfers is that the latter only contribute through the exchange amplitude. 
For the case of a Serber interaction and isoscalar, normal parity transitions (this being a hypothetical situation similar to most of the actual cases considered in this work) a stronger argument can be given. A normal parity transition is defined by the condition $\Delta \pi=(-1)^{\mathrm{J} l}$ where $J_{\ell}$ is the lowest allowed J-transfer. For this case the dominant normal transfer is specified by the triad $\left(\mathrm{J}_{\ell} \mathrm{OJ}_{\ell}\right)$ and the corresponding non-normal transfers are specified by $\left(J_{\ell} \pm l, I, J_{\ell}\right)$. An isoscalar transition proceeds through the $\mathrm{T}=0$ multipoles of the interaction. For a serber interaction $t_{00}$ is three times stronger than $t_{10}$ which introduces at least a factor of nine difference in magnitude between normal and non-normal transfers. In addition collective effects in the target nuclei will be displayed in (JOJ) triads. For the case of an abnormal parity transition, i.e. $\Delta \pi=(-1)^{\mathrm{J}} \ell^{+1}$, the factor of nine goes the other way. Neglecting the non-normal transfers may be serious here.

By expanding $t^{E}\left(\left|\bar{k}_{1}-\bar{k}_{2}\right|\right)$ in Eq. (5I) in a Taylor series about $\lambda_{0}^{2}$, additional terms can be included in $\widetilde{E}^{L S J}\left(r_{0}\right)$. These will correct for the finite spread of momentum components in the distorted and bound state wave functions and wili introduce some dependence on the local momentum transfers. In principle this series conserves the possibility of non-normal transfers. It is presently being studied only with the hope of improving the results for normal transfers. The series is developed formally in Appendix A, but will not be discussed in this paper. 
The t-matrix for free two-nucleon scattering is a function of $q^{2}, p^{2}$, and $\bar{q} \cdot \bar{p}$. The dependence on $\bar{p}$ is related to the fact that it includes the effect of exchange scattering. The pseudo-potential used in this work is determined from those components of the free two-nucleon t-matrix which are off the two-nucleon energy shell as prescribed above, i.e. Eq. (53). On the average, exchange scattering is thus being treated in essentlaliy the same way. The pseudo-potential is strongly energy dependent. It might have been better to include only the effects of direct scattering in this pseudo-potential and treat antisymmetrization in a consistent way throughout.

7. Transition Densities

It is convenient to introduce the transition densities.

These are

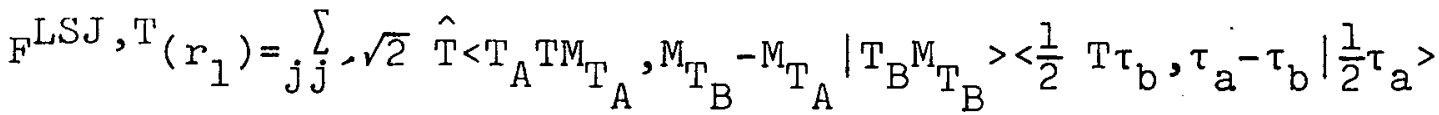

$$
\begin{aligned}
& x S\left(J_{A} J_{B} J^{\prime} T_{A} T_{B} T^{\prime} ; j^{\prime}\right) \\
& \left.x<j-\frac{1}{2}|| \frac{\delta\left(r_{1}-r_{2}\right)}{r_{1}^{2}} T^{L S J}(2) \tau^{T}(2)|| j \frac{1}{2}\right\rangle
\end{aligned}
$$

and

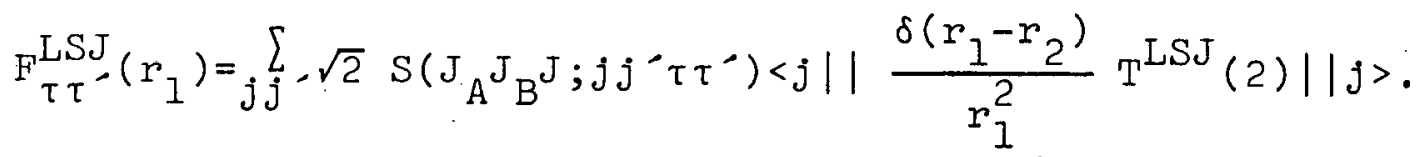

Deleting reference to the fractional parentage expansion these relations can be rewritten as 


$$
\begin{aligned}
& { }_{1} \mathrm{LSJ}^{\mathrm{LST}}\left(\mathrm{T}_{1}\right)=\sqrt{2} \hat{\mathrm{T}}<\mathrm{T}_{\mathrm{A}} \mathrm{TM}_{\mathrm{T}_{\mathrm{A}}}, \mathrm{M}_{\mathrm{T}_{\mathrm{B}}}-\mathrm{M}_{\mathrm{T}_{\mathrm{A}}}\left|\mathrm{T}_{\mathrm{B}} \mathrm{M}_{\mathrm{T}_{\mathrm{B}}}><\frac{1}{2} \mathrm{~T}_{\mathrm{b}}, \tau_{\mathrm{a}}-\tau_{\mathrm{b}}\right| \frac{1}{2} \tau_{\mathrm{a}}> \\
& \left\langle J_{B} T_{B}\left\|\sum_{i=1}^{N} \frac{\delta\left(r_{1}-r_{i}\right)}{r_{I}^{2}} T^{L S J}(i) \tau^{T}(i)\right\| J_{A} T_{A}\right\rangle
\end{aligned}
$$

and

$$
\mathrm{F}_{\tau \tau^{-}}^{\mathrm{LSJ}}\left(r_{1}\right)=\sqrt{2}<J{ }_{B}||_{i}^{\sum_{i}^{\prime}} \frac{\delta\left(r_{I}-r_{i}\right)}{r_{I}^{2}} T^{L S J}(i)|| J_{A}>\text {. }
$$

'l'he sum on $i$ in Eq. (58') runs over all target nucleons while in Eq. (59') the sum on i runs only over those target nucleons consistent with the subscript $\tau \tau^{\prime}$ on $\mathrm{F}_{\tau \tau^{-}}^{\mathrm{LSJ}}\left(\mathrm{r}_{1}\right)$. For example, in the $\left(p, p^{\prime}\right)$ reaction this sum would run over either target protons or target neutrons. The form factors are related to the transition densities by the following expressions.

$$
\begin{aligned}
& \tilde{F}^{\mathrm{LSJ}}\left(r_{0}\right)=\sum_{\mathrm{T}} \int \mathcal{V}_{\mathrm{STL}}\left(\mathrm{r}_{0} ; r_{1}\right) \mathrm{F}^{\mathrm{LSJ}, \mathrm{T}}\left(\mathrm{r}_{1}\right) \mathrm{r}_{1}{ }^{2} \mathrm{dr} \mathrm{r}_{1} \\
& \tilde{F}^{L S J}\left(r_{0}\right)=\sum_{\tau \tau}-\int \nu_{S \tau \tau}{ }^{\prime}\left(r_{0} ; r_{1}\right) F_{\tau \tau}^{L S J}-\left(r_{1}\right) r_{1}{ }^{2} d r_{1}
\end{aligned}
$$

In Eq. (58") and Eq. (59") $\mathcal{V}_{\mathrm{L}}\left(\mathrm{r}_{0} ; \mathrm{r}_{\mathrm{l}}\right)$ represents either the appropriate multipole coefficient of the impulse approximation pseudo-potential or

$$
t_{L}\left(r_{0} ; r_{1}\right)+A(I)\left(\lambda_{0}^{2}\right) \frac{\delta\left(r_{0}-r_{1}\right)}{r_{0}^{2}}
$$

when the $K-K$ or effective range forces are used.

Note that in introducing the transition densities an additional partition of the inelastic nucleon-nucleus 
scattering calculations has been achieved. The first separated the details of the interaction model and nuclear structure from the distorted wave calculation. Here the details of structure are separated from the radial form of the interaction and the effects of antisymmetrization.

Detailed formulae for calculating $\bar{F}^{\mathrm{LSJ}}\left(r_{0}\right)$ and $\dot{F}^{\mathrm{LSJ}}\left(r_{I}\right)$ for the cases of interest in this work are given in Appendix B. The manner in which the transitions densities are related to the inelastic electron-nucleus scattering form factors and the reduced matrix elements for $\gamma$-transitions is discussed in Appendix $C$. This is important as it provides the means for calibrating the nuclear wave functions used in testing the interaction models in this work. The relation of the transition densities to these reactions is independent of the approximations involved in treating inelastic nucleonnucleus scattering in the local D.W.A. 


\section{CHAPTER 3}

\section{IMPULSE APPROXIMATION PSEUDO-POTENTIAL}

The free two nucleon scattering amplitude has the form 33

$m=A+B \bar{\sigma}_{0} \cdot \hat{n} \bar{\sigma}_{1} \cdot \hat{n}+C\left(\bar{\sigma}_{0}+\bar{\sigma}_{1}\right) \cdot \hat{n}+E \bar{\sigma}_{0} \cdot \hat{q} \bar{\sigma}_{1} \cdot \hat{q}+F \bar{\sigma}_{0} \cdot \hat{p} \bar{\sigma}_{1} \cdot \hat{p}$

where $\hat{q}=\bar{q} /|\bar{q}|, \bar{q}=\bar{k}^{\prime}-\bar{k} ; \hat{n}=\bar{n} /|\bar{n}|, \bar{n}=\bar{k} \times \bar{k}^{\prime} ;$ and $\hat{p}=\hat{q} \times \hat{n}$. Here $\bar{k}$ and $\bar{k}^{\prime}$ are the initial and final relative momenta of the two nucleons and $\bar{q}$ is the momentum transfer. The unit vectors $(\hat{q}, \hat{n}, \hat{p})$ form a right handed coordinate system and the coefficients $A, B, C, E$, and $F$ are functions of $q^{2}, q^{2}+p^{2}$, and $\bar{q} \cdot \bar{p}$ as well as iso-spin, i.e.

$$
A=\frac{1}{4}\left(3 A_{1}+A_{0}\right)+\frac{1}{4}\left(A_{1}-A_{0}\right) \bar{\tau}_{0} \cdot \bar{\tau}_{1}
$$

where $A_{0}$ is the coefficient for the singlet $i-s p i n$ state and $A_{1}$ is the coefficient for the triplet i-spin state. The free two-nucleon $t$-matrix is related to $m$ by

$$
t=-\frac{4 \pi \hbar^{2}}{M} m
$$

Note that $M$ can be written as follows

$$
m=A+\frac{1}{3}(B+E+F) \bar{\sigma}_{0} \cdot \bar{\sigma}_{1}+\text { other terms }
$$


where the other terms are the parts of the scattering amplitude which are not scalar in spin space. The components of $m$ have been calculated ${ }^{32}$ from the H-J potential using nucleon-nucleus kinematics (as prescribed in Section 6 of Chapter 2) for lab energies of $19.6,27.5,40,50,60,95$, 125 , and $155 \mathrm{MeV}$. This gives $m$ as a function of $q^{2}, E_{L A B}$, and $N$. The dependence on $N$ is weak which is evident from Eq. (2.53) and only the $N=12$ results are used in this work.

A typical set of results are shown in Fig. I which shows the real part (the free two nucleon scattering amplitude is, of course, complex) of $\frac{1}{4}\left(3 A_{1}+A_{0}\right)$ as a function of a for $\mathrm{E}_{\mathrm{LAB}}=19.6,50$, and $125 \mathrm{MeV}$. The components of the scattering amplitude which are not scalar in spin space are small for lab energies below $100 \mathrm{MeV}$. This is good as this study is restricted to those components of the interaction which can be expressed in the form of Eq. (2.18). The pseudo-potential is obtained simply by inserting the first two terms on the right in Eq. (4) into Eq. (3) and taking the Fourier transform of the resulting relation. As a matter of convenience a Yukawa radial form, i.e. $\mathrm{Ve}^{-\mathrm{mr}} / \mathrm{mr}$ has been assumed for the components of the pseudo-potential. Ihis is tantamount to fitting the components of $M\left(q^{2}\right)$ to

$$
-\frac{M}{\hbar^{2}} \frac{V}{m}\left(m^{2}+q^{2}\right)^{-1}
$$

The strength and range parameters of the various components of $t\left(\bar{r} ; E_{L A B}\right)$ are read off graphs of the form of Fig. 1 . 


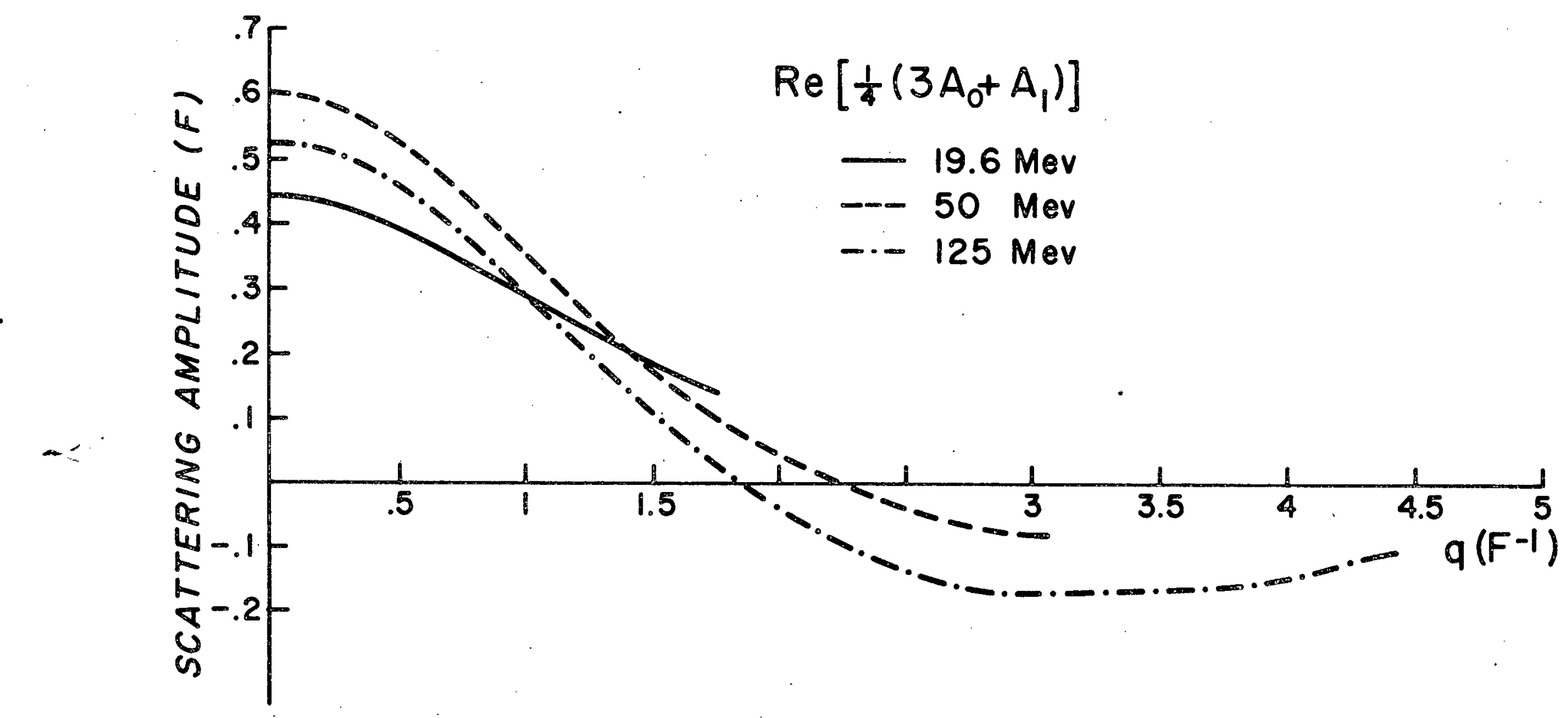

Figure 1.--Real part of the $\frac{1}{4}\left(3 A_{1}+A_{1}\right)$ component of the free two-nucleon scattering amplitude as a function of $q$. The calculation is off the energy shell with $N=12$. 
The range $m$ is determined roughly by the half maximum and the quantity $\frac{\mathrm{V}}{\mathrm{m}^{3}}$ is proportional to the value of the scattering amplitude in the forward direction $(\bar{q}=0)$. The constant of proportionality is $-41.5 \mathrm{MeV} \cdot \mathrm{F}^{2}$ with the scattering amplitude given in $\mathrm{F}$ and $\frac{\mathrm{V}}{\mathrm{m}^{3}}$ given in $\mathrm{MeV} \cdot \mathrm{F}^{3}$.

The strength and range parameters for the spin, ispin components and $p-p \cdot(n-n)$ and $n-p$ spin components of the pseudo-potential are given in Table 1 for $\mathrm{E}_{\mathrm{LAB}}=20-60$ MeV. T'wo Yukawa fits to the scattering amplitude have also been made although they have not been used in any calculations. Unlike the one Yukawa fits which only fit the scattering amplitude closely in the forward direction, these fit it quite well over the entire range of $q$ displayed in Fig. 1. The parameters for these two Yukawa fits are available but will not be given here.

Inspection of Table 1 shows that the pseudo-potential has a large imaginary part. The real part of $\frac{1}{4}\left(A_{1}-A_{0}\right)$ and both the real and imaginary parts of the spin-flip, non-ispin-filp part of the scattering amplitude vary quite strongly with energy and are not fit very well by the Yukawa function. The former indicates large non-localities and the latter indicates that the two Yukawa fits should probably be used in these cases. Neglecting these difficulties for $\frac{1}{4}\left(A_{1}-A_{0}\right)$ is not serious because the imaginary part, which is comparable to the real part, is fairly well determined.

For the purpose of facilitating comparison of the various components of the pseudo-potential with each other 
TABLE 1.--Strength and range parameters for components of 1 mpulse approximation pseudo-potent1al, $\mathrm{S}$ is $\mathrm{V} / \mathrm{m}^{3}$ in $\mathrm{MeV} \cdot \mathrm{F}^{3}$ and $\mathrm{m}$ is inverse range in $\mathrm{F}^{-1}$. Two numbers appear in each column. The first is for the real part of the pseudopotential and the second, in parenthesis, is for the imaginary part.

\begin{tabular}{|c|c|c|c|c|c|c|c|c|}
\hline$E_{\text {LAB }}[\mathrm{MeV}]$ & \multicolumn{2}{|c|}{$\mathrm{V}_{00}$} & \multicolumn{2}{|c|}{$\cdot v_{10}$} & \multicolumn{2}{|c|}{$\mathrm{V}_{01}$} & \multicolumn{2}{|c|}{$v_{11}$} \\
\hline 20 & $-19.5(-52.4)$ & $1.34(1.59)$ & $12.4(8.30)$ & $2.50(1.58)$ & $-6.06(24.9)$ & $.950(1.83)$ & $9.57(16.6)$ & $.782(1.68)$ \\
\hline 30 & $-24.6(-41.1)$ & $1.22(1.52)$ & $8.67(4.72)$ & $2.50(1.50)$ & $-.960(19.9)$ & $.880(1.78)$ & $10.8(12.2)$ & $.818(1.68)$ \\
\hline 40 & $-24.1(-33.4)$ & $1.16(1.46)$ & $6.19(.023)$ & $2.50(1.65)$ & $1.12(15.7)$ & $2.50(1.65)$ & $11.6(9.54)$ & $.808(1.68)$ \\
\hline 50 & $-24.9(-28.4)$ & $1.15(1.39)$ & $4.56(.015)$ & $2.50(1.88)$ & $2.37(13.9)$ & $2.50(1.53)$ & $11.8(7.68)$ & $.780(1.60)$ \\
\hline 60 & $-25.0(-25.2)$ & $1.15(1.31)$ & $3.29(.005)$ & $1.30(1.95)$ & $2.70(11.9)$ & $2.50(1.43)$ & $12.0 .(6.23)$ & $.750(1.55)$ \\
\hline $\mathrm{E}_{\mathrm{LAB}}[\mathrm{MeV}]$ & \multicolumn{2}{|c|}{$\mathrm{V}_{\mathrm{pp}}^{0}$} & \multicolumn{2}{|c|}{$\mathrm{V}_{\mathrm{pp}}^{1}$} & \multicolumn{2}{|c|}{$v_{p n}^{0}$} & \multicolumn{2}{|c|}{$\mathrm{v}_{\mathrm{pn}}^{1}$} \\
\hline 20 & $-24.4(-27.6)$ & $1.16(1.47)$ & $22.2(25.3)$ & $1.94(1.63)$ & $-13 \cdot 3(-75 \cdot 5)$ & $1.80(1.60)$ & $3.32(-8.20)$ & $2.50(1.84)$ \\
\hline 30 & $-23.2(-20.7)$ & $1.10(1.40)$ & $19.5(21.8)$ & $1.42(1.69)$ & $-21.2(-62 \cdot 3)$ & $1.45(1.58)$ & $-2.08(-7.45)$ & $.245(1.72)$ \\
\hline 40 & $-22.8(-16.7)$ & $1.19(1.32)$ & $17.8(12.9)$ & $1.16(1.70)$ & $-25.2(-49.8)$ & $1.32(1.50)$ & $-5.24(-6.65)$ & $.420(1.60)$ \\
\hline 50 & $-22 \cdot 7(-14 \cdot 5)$ & $1.06(1.22)$ & $16.4(9.12)$ & $.960(1.72)$ & $-27 \cdot 1(-42 \cdot 3)$ & $1.24(1.38)$ & $-7.25(-6.23)$ & $.550(1.40)$ \\
\hline 60 & $-22.4(-13.4)$ & $1.08(1.22)$ & $15.6(7.05)$ & $.880(1.66)$ & $-28.0(-36.9)$ & $1.23(1.30)$ & $-8.72(-5.60)$ & $.650(1.30)$ \\
\hline
\end{tabular}


and with corresponding components of other interactions real IF range Yukawa "equivalent" to this interaction has been determined over the energy region from 20 to $60 \mathrm{MeV}$. This is given in Table 2. The real $1 \mathrm{~F}$ range Yukawa form has been selected as it is the form which has been popular in recent analyses. This "equivalent" interaction is no more than a rough representation of the actual pseudopotential, i.e. in a calculation it won't reproduce precisely the multipole and state dependence of the prototype. From the table it is seen that the pseudo-potential is similar to a Serber force and the strengths of the components decrease fairly rapidly with energy. The latter effect is a direct result of the decreasing importance of the exchange component of the scattering amplitude.

TABLE 2.--Strengths for real IF range Yukawa "equivalent" to impulse approximation pseudo-potential. All values are in MeV.

\begin{tabular}{cccccccccc}
$E_{L A B}$ & $V_{00}$ & $V_{10}$ & $V_{01}$ & $V_{I I}$ & $V_{p p}^{0}$ & $V_{p p}^{1}$ & $V_{n p}^{0}$ & $v_{n p}^{I}$ \\
\hline 20 & -86.9 & 33.6 & 45.9 & 38.3 & -53.2 & 59.5 & -123 & -17.2 \\
30 & -69.3 & 23.1 & 35.4 & 29.5 & -38.6 & 46.1 & -103 & -12.8 \\
40 & -56.3 & 15.5 & 26.0 & 20.5 & -34.9 & 30.1 & -81.8 & -10.9 \\
50 & -48.8 & 11.4 & 22.1 & 15.9 & -29.8 & 22.2 & -67.4 & -9.6 \\
60 & -43.8 & 4.3 & 18.3 & 13.6 & -25.5 & 18.0 & -59.1 & -9.2 \\
\hline
\end{tabular}


CHAPTER 4

\section{THE PROJECTILE-TARGET INTERACTION}

By analogy with the bound state problem the two-body interaction to be used in nucleon-nucleus scattering calculations is given by the integral equation

$$
t=v-v \frac{Q}{e-i \varepsilon} t
$$

where $\mathrm{V}$ is the nucleon-nucleon potential, $Q$ is the Pauli operator, and $e$ is the energy denominator defining the many body Green's function - defined in accord with the conventions of Kuo and Brown. ${ }^{1,20}$ The presence of the $i \varepsilon$ in $\mathrm{Eq}$. ( 1 ) makes $t$ complex. It is possible to express $t$ in terms of the operator

$$
t_{B}=v-v \frac{Q}{e} t_{B}
$$

which is real. This expression is

$$
t=t_{B}-i \pi t_{B} Q \delta(e) t
$$

If the imaginary part of $t$ is small, and from the deformed optical potential description of inelastic nucleon-nucleus scattering (see Section 2 of Appendix B) it is expected to 
be small with respect to the real part in the medium energy region, $\mathrm{Eq}$. (3) can be approximated as

$$
t \sim t_{B}-i \pi t_{B} Q \delta(e) t_{B}
$$

This argument, which is based on the relative magnitude of the real and imaginary part of the inelastic scattering form factors given by that model, is valid only in the region of the target nucleus where the form factor is appreciable.

Eq. (2) formally is equivalent to the definition for the bound state reaction matrix, 1,20 but it must be remembered that the energy denominator, e, appropriate for the scattering problem is not the same as that for the bound it tate problem. Kuo and Brown have soived Eq. (2) for the bound state problem ${ }^{1,20}$ taking the $\mathrm{H}-\mathrm{J}$ potential for the nucleon-nucleon interaction. Using the Scott-Moskowski separation method, 47 they have shown that the attractive, even components of $t_{B}$ are well represented by

$$
t_{B}^{\sim} v_{\ell}-v_{T \ell} \frac{Q}{e} v_{T \ell}
$$

Where $v_{\ell}$ is the long range part of the $H-J$ potential and $V_{\text {re }}$ is the long range part of the tensor component of this potential.

The second term in Eq. (4) only acts in triplet states and is given approximately by 


$$
-\mathrm{v}_{\mathrm{T} \ell} \stackrel{\mathrm{Q}}{\mathrm{e}} \mathrm{v}_{\mathrm{Tl}} \approx-\frac{8}{\langle e\rangle} \mathrm{v}_{\mathrm{T} \ell}^{2}(\mathrm{r})+\frac{2}{\langle e\rangle} \mathrm{v}_{\mathrm{T} \ell}^{2}(r) \mathrm{S}_{12}
$$

where $\mathrm{v}_{\mathrm{T} \ell}(r)$ is the radial part of the long range part of tensor component of the H-J potential, $\mathrm{S}_{12}$ is the "tensor" operator, and <e> is a mean energy denominator which is. highly state dependent. The state dependence of $\langle e\rangle$ will be discussed in a moment. The first term on the right in Eq. (5) gives a very important contribution to the central, triplet even component of $t_{B}$ while the second term gives a small (10\%) contribution to the even tensor component of $t_{B}$. In writing $\mathrm{Eq}$. (4) several terms in the scott-Moskowski expansion have been omitted. They consist principally, for the H-J potential, of a contribution $t_{S}$ from the repulsive core and various second order terms including a cross-term between $t_{S}$ and $v_{\ell}$. These additional terms are state dependent, but their net effect is small. They will be ignored. Note that Eq. (4) comprises a local interaction in configuration space.

The odd components of the nucleon-nucleon potential are repulsive; therefore, the corresponding components of $t_{B}$ can not be obtained from the Scott-Moskowski expansion since it does not exist. Kuo and Brown use the reference spectrum method ${ }^{1,20}$ to treat the odd components. This does not yield a configuration space interaction. In any event, $t_{B}$ is repulsive in singlet odd states and has some attractive triplet odd matrix elements. In binding energy calculations, 
the net effect of these odd state interactions is negligible, therefore, it is concluded that the average effect of the odd state interactions is small.

The contribution to the triplet even component of $t_{B}$ contained in Eq. (5) is state dependent due to its dependence on $<e>$. Equivalently it is density dependent. The mean energy. denominator $<e>$ is state dependent because of its connection to the Pauli operator which appears on the left in $\mathrm{Eq}$. (5), i.e. as the effect of $Q$ is reduced as the density decreases, the strong tensor interaction between relative $\mathrm{s}$ and $d$ states is felt more strongly and this must be accounted for by a decrease in <e>. This effect is very clear in nuclear matter calculations. At low density contributions to the binding energy from relative ${ }^{3} S_{1}$ states are considerably larger than those from the ${ }^{{ }} S_{0}$ state, showing the full strength of the tensor force. At observed densities the two contributions are about equal. For high densities the ${ }^{1} \mathrm{~S}_{0}$ contribution is the greater - an effect which is an important aid to nuclear saturation.

An estimate of this effect can be obtained by comparing calculations of the bound state matrix elements for two free nucleons in a nucleus, without the presence of other nucleons, and with those where the presence of other nucleons is taken into account. In the first case, taking $0^{16}$ as an example, ${ }^{66}$ the ${ }^{3} \mathrm{~S}_{1}$ matrix elements are $-16 \mathrm{MeV}$, while with the Pauli Principle taken into account they are $\sim 9 \mathrm{MeV}$. The ${ }^{{ }^{1} \mathrm{~S}_{0}}$ matrix elements are $\sim 8 \mathrm{MeV}$ and very quite slowly with 
density. Thus the average s-state matrix element, which is by far, the largest, varies from $\sim 81 / 2 \mathrm{MeV}$ in the nuclear interior to $\sim 12 \mathrm{MeV}$ far outside the nucleus.

This somewhat lengthy discussion of the bound state reaction matrix has been given with a view towards assuming that it is equivalent to $t_{B}$ for the scattering problem, i.e. differences between the propagator of Eq. (2) for the bound state problem and the scattering problem (in the. energy region of interest here) do not alter $t_{B}$ appreciably. The stability of the separation distances (they remain essentially constant up to $30 \mathrm{MeV}$ in the two-nucleon center of mass) for the important even components of $t_{B}$ supports this hypothesis. With this assumption, near the target

$$
\begin{aligned}
& t^{E}=t_{B}^{E}-i \pi t_{B}^{E} Q \delta(e) t_{B}^{E} \\
& t^{O}=0
\end{aligned}
$$

where the superscripts $E$ and $O$ stand for even and odd, respectively, and

$$
\begin{aligned}
\mathrm{t}_{\mathrm{B}}^{\mathrm{E}} & =\mathrm{v}_{\ell}^{\mathrm{T}}-\frac{8}{\langle e\rangle} \mathrm{v}_{\mathrm{T} \ell}^{2}(r) & & \text { (triplet states) } \\
& =\mathrm{v}_{\ell}^{\mathrm{S}} & & \text { (singlet states) }
\end{aligned}
$$

where the superscripts $T$ and $S$ denote triplet and singlet, respectively.

In writing Eq. (6) the odd state components of $t$ are being neglected and in writing $\mathrm{Eq}$. (7) the second order 
contribution to the tensor force has been dropped. The state dependence of the triplet component of $t_{B}^{E}$ could be incorporated in Eq. (7) by defining <e> to be a function of the Jocal density.

Now consider the region far outside the target nucleus where the density is low and the effect of other nucleons is negligible. Here the propagator in Eq. (1), Q/e, becomes the propagator for two free nucleons, $1 / e_{0}$, and $t$ is given locally by $t_{i m p u l s e}$ i.e. the pseudo-potential given in Chapter 3 which was derived from the free two-nucleon scattering amplitude. The tensor force now makes itself felt with full strength, but not in the real part of the ilteraction. The approximation of $\mathrm{Eq}$. ( $3^{\prime}$ ) is no longer valid, and the optical theorem forces the strength into the imaginary part of the interaction. The large imaginary component of the pseudo-potential is evident in Table 1 shown in Chapter 3.

Combining. these local arguments leads to a picture of a force which is primarily real inside the nucleus where the effect of the tensor in generating an effective central force is somewhat damped, going over to the impulse approximation at large distances, i.e. a force which has a large imaginary component. This asymptotic region is, however, likely to be at a density where all form factors are quite negligible. That is to say the picture of the force in the region of the target, which is where the scattering takes place, is of prime importance. In summary, near the target $t$ 
is expected to be complex and density dependent. The real part is expected to increase outside the nuclear surface by about $50 \%$ on the average; the imaginary part to be quite small in the interior, peaked outside the nuclear surface, as all the form factors involved in evaluating $t_{B}^{E} Q \delta(e) t_{B}^{E}$ are peaked at the nuclear surface, but small for incident energies up to about $40 \mathrm{MeV}$. At much higher energies this is not true.

As the incident energy increases then the difference between $\frac{\mathrm{Q}}{\mathrm{e}}$ and $\frac{\mathrm{l}}{\mathrm{e}_{0}}$ becomes less important and the impulse approximation becomes valid. However it should be pointed out that this approach asymptotically at high energies is quite slow. The impulse approximation is still a poor approximation at $150 \mathrm{MeV}$, even though it predicts crosssections correctly. Its order of magnitude is quite good, but it's phase, i.e. the relative strength of the real and imaginary part of the interaction, is quite wrong as is shown by the fact that the ground state expectation value of $t_{i m p u l s e}$ does not give the optical potential (real and imaginary part), and that variables likely to be sensitive to the phase, like polarization, are by no means predicted successfully. $40-43$ It works better at $1 \mathrm{GeV}$, though the tests then are not as stringent. 44 Therefore, $t$ is to approach $t_{\text {impulse }}$ only slowly for the energy region we are considering. On the other hand, as far as its magnitude is concerned, disregarding its phase, the impulse approximation might not be too far out. 
The arguments which have been presented above are very rough and, in fact, they could be wrong in detail. They are intended more as a suggestion than actual truth. The resolution of the points which have been made is a problem related to, but separate from, the purpose of this work which is to determine whether or not one make some sense out of inelastic nucleon-nucleus scattering using the interactions which are already available and convenient to use in D.W.A. calculations. These are, of course, the impulse approximation pseudo-potential and the interaction defined in Eq. (6) and Eq. (7). It should also be mentioned that an essentially identical discussion of $t$ has been given, independently, by Satchler 67 He has aiso made some estimates of the imaginary, part of $t$.

The Kallio-Kolltveit potential is an s-state potential with triplet even and singlet even components defined by 46

$$
\begin{array}{rlrl}
V_{k k}^{i}(r) & =+\infty & r \leq c \\
& =-A_{i} e^{-\alpha_{i}(r-c)} & & r>c
\end{array}
$$

where $A_{T}=475.0 \mathrm{MeV}, \alpha_{\mathrm{T}}=2.5214 \mathrm{~F}^{-1}, A_{\mathrm{S}}=330.8 \mathrm{MeV}, \alpha_{\mathrm{S}}=2.4021 \mathrm{~F}^{-1}$, and $c=0.4 \mathrm{~F}$. The long range part of this potential is known to give a good representation the central components of $t_{B}^{E}$ as defined in Eq. (7). In the calculations of this work, the non-central parts and the imaginary part of $t_{B}^{E}$ are neglected and the $K-K$ force is taken to represent its central part of $t_{B}^{E}$. Fixed separation distances, $d_{S}=1.025 F$ and $d_{T}=0.925 F$, are used throughout. The $K-K$ force acts only in relative 
s-states, but since this is an inconvenient restriction for D.W.A. calculations it is allowed to act in all even states. This leads to a slight overestimation of $t_{\mathrm{B}}^{\mathrm{E}}$. Density dependent versions of the $K-K$ force have been proposed by Green. These account for the variation of $t_{B}^{E}$ with <e> in Eq. (7).68 These forces are not examined in this paper.

In lowest order calculations, all of the bound state forces discussed here are found to give a reasonable account of the real part of the optical potential in the medium energy region; therefore, at least the spin, isospin averages of the monopole components of these forces are adequate for the scattering problem.31,32 In detail the $\mathrm{K}-\mathrm{K}$ force gives larger well depths, smaller mean square radii, and somewhat poorer agreement with phenomenological potentials than do the other forces. A reasonable estimate of the imaginary part of the optical potential has also been obtained with these forces. The impulse approximation pseudo-potential failed to describe the optical potential in that it gives too small a real component and a very large imaginary component, i.e. its phase is incorrect.

A real $I F$ range Yukawa "equivalent" to the $K-K$ force (A) has been determined. It is compared with other "equivalent" interactions in Table 1 . These are the impulse approximation pseudo-potential for $E_{L a b}=60 \mathrm{MeV}(B)$, the empirical interaction of $\mathrm{Ball}$ and Cerny ${ }^{69}$ determined from studies of the $\left(\mathrm{He}^{3}, \mathrm{He}^{31}\right)$ and $\left(\mathrm{He}^{3}, t\right)$ reactions in $1 p$-shell nuclei ( $C$ ), the interaction used by Glendenning and Veneroni ${ }^{4}$ in studies of the $\mathrm{Ni}$ isotopes in the ( $\left.p, p^{\prime}\right)$ reaction (D), and the interaction used by $\operatorname{True}^{70}$ in $\mathrm{N}^{14}$ shell 
model calculations ( $E)$. The agreement between the forces is almost complete. The lab energy of $60 \mathrm{MeV}$ was selected for timpulse, because the implicit exchange contribution to this force should be diminished here. Note that only the magnitude of the strengths for interaction $C$ are given. The analysis did not give any conclusive information as to the actual exchange mixture of the force. Further, a guess of the magnitude of enhancement effects in the target nuclei was used in arriving at the value of $v_{00}$ for force $C$. These effects are considerably smaller in $1 p-$ shell nuclei than they are in heavier elements. The overall agreement of these forces is very satisfactory.

TABLE 1.--Comparison of strengths of various real IF range Yukawa "equivalent" interactions. All values are in MeV. A is the $K-K$ force, $B$ is timpulse at $E_{L A B}=60 \mathrm{MeV}, C$ is the interaction determined Ball and Cerny, $D$ is the interaction of Glendenning and Veneroni, and $E$ is the interaction of True.

\begin{tabular}{lclllllll}
\hline Force & $\mathrm{V}_{00}$ & $\mathrm{~V}_{10}$ & $\mathrm{~V}_{01}$ & $\mathrm{~V}_{11}$ & $\mathrm{~V}_{\mathrm{pp}}^{0}$ & $\mathrm{~V}_{\mathrm{pp}}^{\mathrm{I}}$ & $\mathrm{V}_{\mathrm{pn}}^{0}$ & $\mathrm{~V}_{\mathrm{pn}}^{I}$ \\
\hline $\mathrm{A}$ & -36.2 & 6.30 & 17.8 & 12.1 & -18.4 & 18.4 & -54 & -5.75 \\
$\mathrm{~B}$ & -43.8 & 4.30 & 18.3 & 13.6 & -25.5 & 18.0 & -59.1 & -9.20 \\
$\mathrm{C}^{69}$ & $|30-40|$ & $|11-27|$ & $|21|$ & $|17|$ & - & - & - & - \\
$\mathrm{D}^{4}$ & -40.5 & 6.80 & 20.2 & 13.5 & -20.3 & 20.3 & -60.7 & -6.70 \\
$\mathrm{E}^{70}$ & -41.1 & 7.40 & 20.0 & 13.7 & -21.1 & 21.1 & -61.1 & -6.30 \\
\hline
\end{tabular}


CHAPTER 5

THE APPROXIMATE TREATMENT OF ANTISYMMETRIZATION

In this chapter some results obtained with antisymmetrization treated approximately (as discussed in Section 6 of Chapter 2) are compared with corresponding results obtained with the exchange component of the D.W.A. transition amplitude treated properly. The exact results are due to J. Atkinson and V. Madsen.17-19 A modification of the D.R.C. (Direct Reaction Calculation) code available at Lawrence Radiation Laboratory, Livermore, California has been used in obtaining these results. 71 This code is restricted to interactions with radial dependence which can be easily expressed as a combination of not more than three Yukawa functions. Because of this all comparisons are for interactions with Yukawa radial form. No direct information concerning this approximation, is available for the interaction of primary interest in this work--the $\mathrm{K}-\mathrm{K}$ force. The recently developed non-local D.W.A. code at Oak Ridge National Laboratory has been set up to handle interactions of this type, i.e. which have a "hole" in them, and new results should be forthcoming. 16 
1. Yukawa Function

'the essential ingredient of the approximation under consideration is the Fourier transform of the interaction. For the Yukawa function

$$
\mathrm{V}(\mathrm{r})=\mathrm{Ve}-\mathrm{mr} / \mathrm{mr}
$$

this transform is given by

$$
V\left(\lambda^{2}\right)=(4 \pi V / m)\left(\lambda^{2}+m^{2}\right)^{-1}
$$

I'able 1 gives the value of this transform as a function of the $\mathrm{lab}$ energy for $\mathrm{m}=0.5,1.0,1.5,2.0,2.5$, and $3.0 \mathrm{~F}^{-1}$. $\mathrm{V}$ has been taken to be $1 \mathrm{MeV}$ and it is to be remembered that the $1 \mathrm{ab}$ energy and $\lambda^{2}$ are related by $\lambda^{2}=2 \mathrm{ME} / \hbar^{2}$. The last row in this table gives the ratio of the Fourier transform at $20 \mathrm{MeV}$ witr. respect to that at $80 \mathrm{MeV}$. These energies span the region of interest in this work and this ratio is indicative, within the framework of this approximation, of the relationship between the range of the interaction and the energy dependence of the exchange component of D.W.A. transition amplitude. It is seen that this ratio decreases with the range and is approach-ing one in the zero range limit.

2. Transitions in $\mathrm{Zr}^{90}+\mathrm{p}$

Dependence on Energy and Multipole

The ratio of the exchange integrated cross section to the direct integrated cross section has been given for the $L=0,2,4,6$, and 8 transitions in the $\mathrm{Zr}^{90}\left(\mathrm{p}, \mathrm{p}^{\prime}\right) \mathrm{Zr}^{90 *}$ reaction 
TABLE 1.--Fourier transform of Yukawa interactions of various ranges as a function of the lab energy.

\begin{tabular}{l|llllll}
\hline \multirow{2}{*}{} & \multicolumn{6}{|c}{$\mathrm{V}(\mathrm{E})\left[\mathrm{MeV} . \mathrm{F}^{3}\right]$} \\
\cline { 2 - 7 } $\mathrm{E}(\mathrm{MeV})$ & .5 & 1.0 & 1.5 & 2.0 & 2.5 & 3.0 \\
\hline 0 & 101 & 12.6 & 3.72 & 1.57 & .804 & .465 \\
10 & 34.0 & 8.44 & 3.06 & 1.40 & .746 & .441 \\
20 & 20.5 & 6.36 & 2.60 & 1.26 & .696 & .420 \\
30 & 14.6 & 5.10 & 2.26 & 1.15 & .651 & .400 \\
40 & 11.4 & 4.25 & 1.99 & 1.06 & .613 & .382 \\
50 & 9.33 & 3.65 & 1.79 & .975 & .578 & .366 \\
60 & 7.90 & 3.20 & 1.62 & .906 & .547 & .351 \\
70 & 6.85 & 2.84 & 1.48 & .847 & .520 & .337 \\
80 & 6.04 & 2.56 & 1.36 & .794 & .495 & .325 \\
90 & 5.41 & 2.33 & 1.26 & .748 & .472 & .313 \\
100 & 4.89 & 2.14 & 1.17 & .707 & .451 & .302 \\
\hline V(20) & 3.40 & 2.48 & 1.91 & 1.59 & 1.41 & 1.29 \\
\hline
\end{tabular}

at $18.8 \mathrm{MeV}$ as a function of the inverse range of an interaction of. Yukawa radial form. ${ }^{18}$ For the $L=2$ transition the $\sigma_{\text {ex }} / \sigma_{\text {dir }}$ ratio has been calculated as a function of energy with the range of the force fixed at $2 F .^{19}$ A serber exchange mixture has been assumed, and $j-j$ coupling wave functions for two 
protons in the $\lg _{9 / 2}$ orbit were used to describe the target. ${ }^{\prime}$ The $18.8 \mathrm{MeV}$ optical parameters of Ref. 8 have been used throughout. Results obtained approximately are compared with the exact results in Fig. 1. The exact results are shown as dashed lines and the approximate results are indicated by solid lines. In the lower graph the corresponding results are bracketed and labeled with the L-transfer.

The importance of exchange increases with increasing multipole and with decreasing energy. Note that $\sigma_{\text {ex }} / \sigma$ dir deviates more from 1 , the zero range value, as the range of the force increases. For $\mathrm{L}=6$ and $\mathrm{L}=8 \sigma_{\mathrm{ex}}$ is greater than $\sigma_{\text {dir }}$. The approximate values of $\sigma_{e x} / \sigma_{d i r}$ for $L=4$ are about one, but the exact vaiues are less than one. Qualitatively, the agreement of the approximate results with the exact results is quite good. The approximate results overestimate the exact results except for the case $L=8$. The agreement between the approximate and exact values of $\sigma_{\text {ex }} / \sigma_{\text {dir }}$ improves with increasing energy. There is no pronounced change in the agreement as the force range becomes shorter except for the $\mathrm{L}=8$ case. The approximation is improving with increasing

$6^{+}$and $8^{+}$T'o be more precise the $0^{+}$ground state and $0^{+}, 2^{+}, 4^{+}$, couplings couplings of two $1 \mathrm{~g}_{9} / 2$ protons. The allowed normal transfers are specified by the triads $(J, O, J)$ and $(J, I, J)$ where the transferred $J$ must be the same as the total angular momentum of the final state. The $(\mathrm{J}, I, \mathrm{~J})$ contribution vanishes due to a structure selection rule. Two non-normal transfers, $(\mathrm{J} \pm 1,1, J)$, are also allowed. Only the contributions due to normal transfers are being considered in the following discussion, therefore it is unambiguous to specify each transition by the I-transfer implying the contribution due to the triad $(J, O, J)$. 

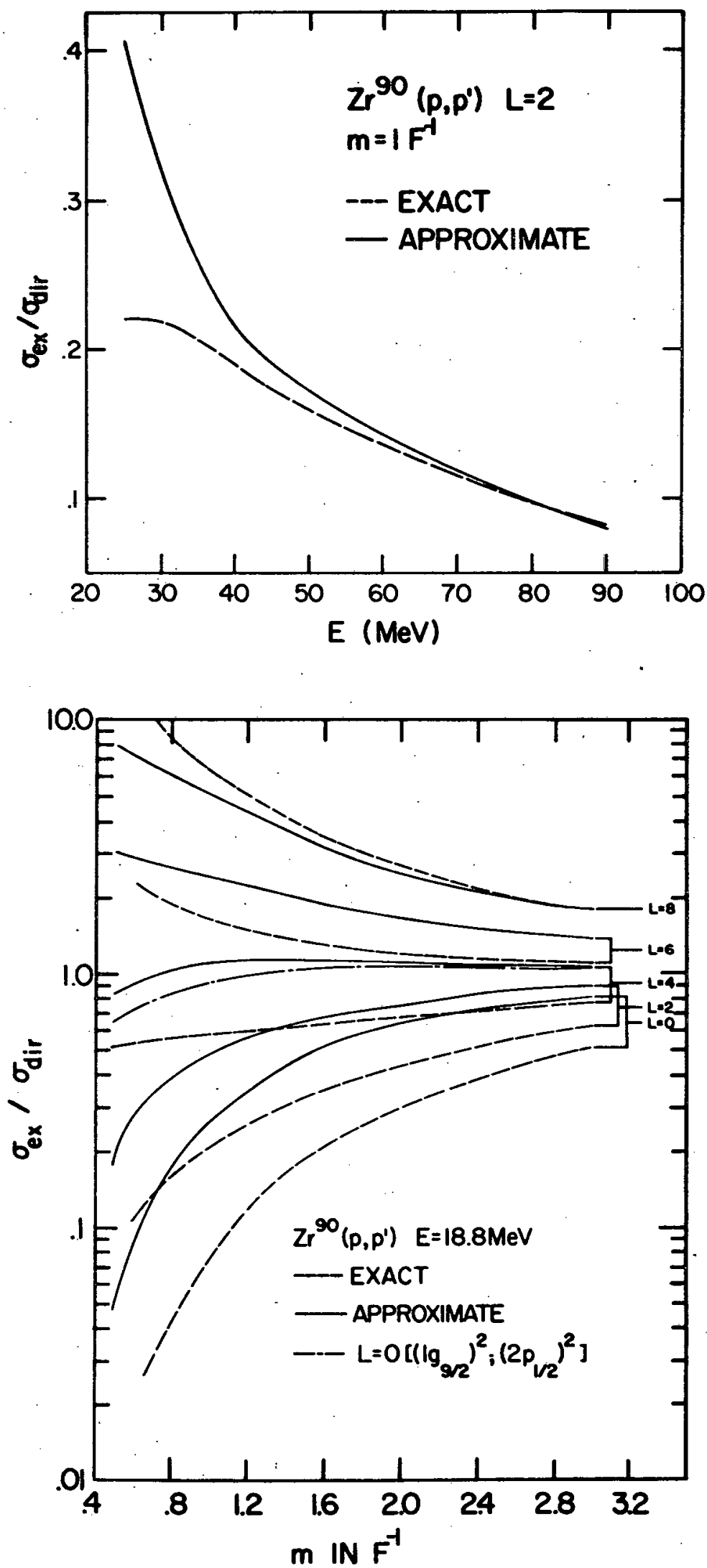

Figure 1.--Comparison of approximate and exact results showing the variation with energy and interaction range of the ratio of the exchange to the direct integrated cross section for several multipoles in the $\mathrm{Zr}^{90}\left(\mathrm{p}, \mathrm{p}^{\prime}\right)$ reaction. 
multipole which is very good since the contribution from the exchange component of the transition amplitude is becoming more important at the same time. The last effect is consistent with the fact that transitions of high multipolarity are not sensitive to the details of the nuclear interior which are ignored in the approximation.

The result indicated by the center line and labeled. $L=0$ in the lower graph of Fig. I is interesting. This approximate result was obtained by considering the ground state and first $0^{+}$state of $\mathrm{Zr}^{90}$ to be described by more realistic configuration mixed wave functions involving both the $1 \mathrm{~g}_{9 / 2}$ and $2 \mathrm{p}_{1 / 2}$ proton levels. 8 The ratio $\sigma_{\mathrm{ex}} / \sigma_{\mathrm{dir}}$ for this case is quite different than the result obtained using only the unrealistic $\left(\lg _{9 / 2}\right)^{2}$ configuration. This indicates that the contribution to cross sections due to exchange can be quite sensitive to the wave functions involved.

Total Cross Section (Direct + Exchange)

If maximum interference between the direct and exchange amplitudes is assumed it follows that the total cross section (direct plus exchange) is given by

$$
\sigma_{\mathrm{T}} \approx\left(\sqrt{\sigma}_{\mathrm{dir}}+\sqrt{\sigma}_{\mathrm{ex}}\right)^{2}=\left(1+\sqrt{\frac{\sigma_{\mathrm{ex}}}{\sigma_{\mathrm{dir}}}}\right)^{2} \sigma_{\mathrm{dir}}=\alpha \sigma_{\mathrm{dir}} .
$$

This assumption is quite good. It has been shown that the direct and exact exchange amplitudes are essentially in phase except for extreme forward and backward angles. ${ }^{18,19}$ This is true to a greater extent in the approximate calculations. Table 2 
displays the values of $\alpha_{\text {Exact }}$ and $\alpha_{\text {Approx }} / \alpha_{\text {Exact }}$ as a function of $\mathrm{L}$ for $\mathrm{m}=8$ and $2.0 \mathrm{~F}^{-1}$. Eq. (3) has been used to determine and $\sigma_{e x} / \sigma_{d i r}$ values have been taken from Fig. 1 . The numbers in the table indicate a maximum error of $40 \%$ in the approximate total cross section. This occurs for $L=0$ and $m=.8 \mathrm{~F}^{-1}$.

To illustrate the rate of improvement of the approximation with increasing energy note that $\alpha_{\text {Approx }} / \alpha_{\text {Exact }}$ goes from 1.15 to 1.02 as $E$ goes from 30 to $50 \mathrm{MeV}$ for the $\mathrm{L}=2$ transition

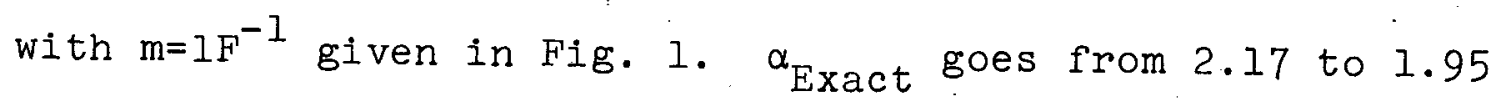
over the same energy region indicating that the enhancement of the direct cross section due to exchange is decreasing fairly slowly with increasing energy for this particular multipole.

TABLE 2.--Comparison of approximate and exact values of $\alpha$, the enhancement of the direct cross section due to exchange, for two values of the interaction range appearing in Fig. I.

\begin{tabular}{|c|c|c|c|c|}
\hline$L$ & $\alpha_{\text {Exact }}$ & $\frac{\alpha_{\text {Approx }}}{\alpha_{\text {Exact }}}$ & $\begin{array}{l}m= \\
\alpha_{\text {Exact }}\end{array}$ & $\frac{\alpha_{\text {Approx }}}{\alpha_{\text {Exact }}}$ \\
\hline 0 & 1.44 & 1.40 & 2.40 & 1.35 \\
\hline 2 & 2.00 & 1.32 & 2.77 & 1.26 \\
\hline 4 & 3.04 & 1.35 & 3.33 & 1.29 \\
\hline 6 & 5.66 & 1.22 & 4.58 & 1.19 \\
\hline 8 & 14.8 & .804 & 6.82 & .978 \\
\hline
\end{tabular}


Fig. 2 shows the direct, exchange, and total integrated cross sections as a function of multipole for the cases $\mathrm{m}=.5 \mathrm{~F}^{-1}$ and $\mathrm{m}=3.0 \mathrm{~F}^{-1}$ of $\mathrm{Fig}$. 1 . Both the approximate and exact results are shown for $\sigma_{\mathrm{ex}}$ and $\sigma_{\mathrm{T}}$ and maximum interference has been assumed in obtaining $\sigma_{\mathrm{T}}$. The absolute normalization of the results is arbitrary, but the relative magnitude of each, for each force range, is as shown. This figure illustrates how $\sigma_{T}$ falls off slower with $L$ than does $\sigma_{\text {dir }}$ due to the contribution from $\sigma_{\text {ex }}{ }^{--a n}$ effect which is not very pronounced for $m=3 \mathrm{~F}^{-1}$--and how the fairly large errors in the approximate values of $\sigma_{e x} / \sigma_{d i r}$ are not so strongly reflected in $\sigma_{\mathrm{T}}$.

Fig. 3 compares the behavior, as a function of multipole, of $\sigma_{\mathrm{T}}$ (Exact) for the $2 \mathrm{~F}$ range force with $\sigma_{\text {dir }}$ for forces with $\mathrm{m}=1.0$ and $1.5 \mathrm{~F}^{-1}$. The behavior is similar. A previous empirical analysis of these transitions, in which antisymmetrization was ignored, led to the conclusion that a $1 F$ range Yukawa interaction reproduced the observed multipole dependence of the cross sections. ${ }^{8}$ A longer range would have been selected had antisymmetrization been taken into account.

\section{Angular Distributions}

The direct (D) and approximate exchange (E) angular distributions for the $2 \mathrm{~F}$ range Yukawa force are shown in Fig. 4. All curves have been normalized to one at peak. With the exception of the $L=0$ transition the exchange angular 

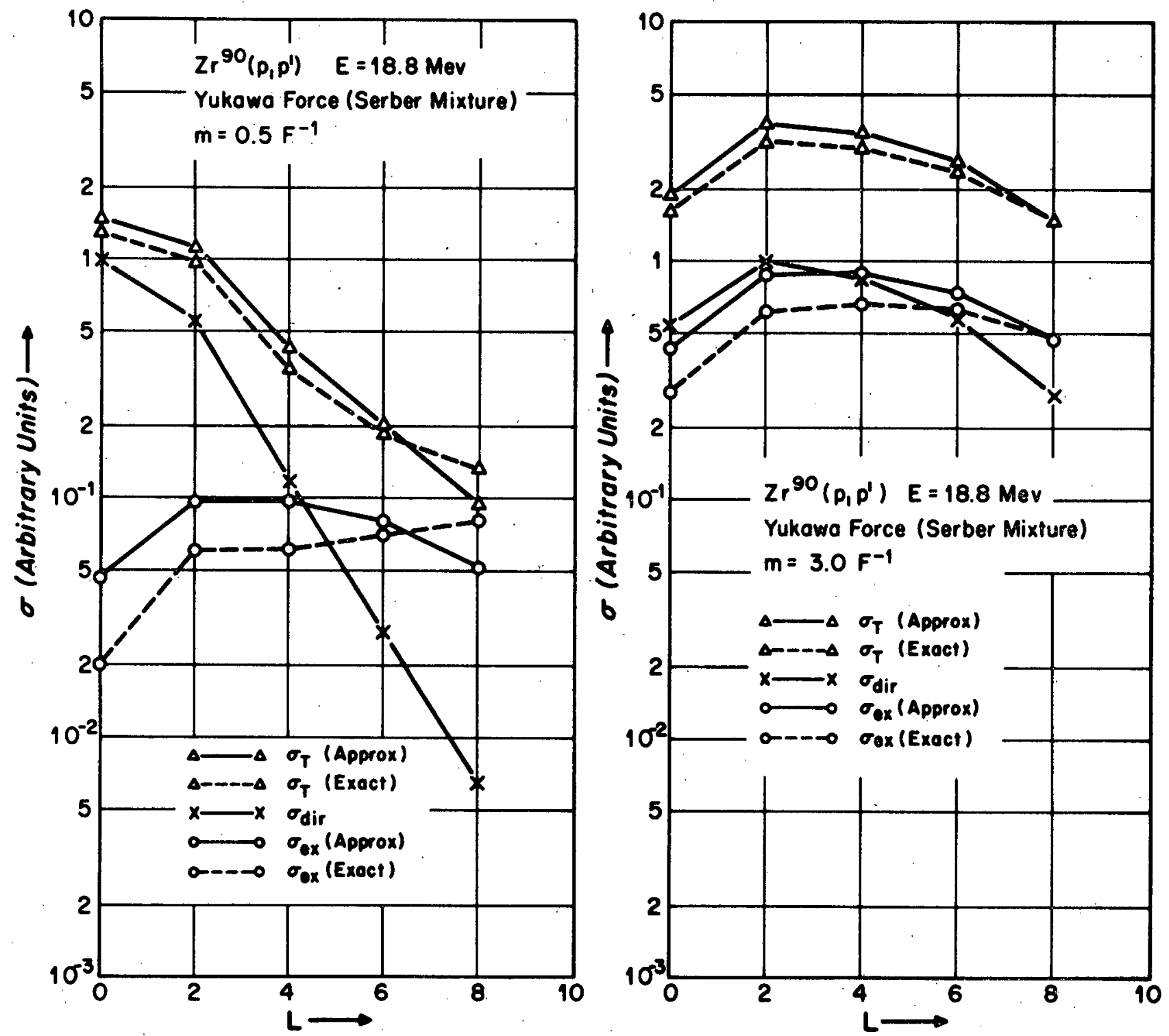

Figure 2.--Comparison of $\sigma_{\mathrm{d} 1 \mathrm{r}}, \sigma_{\mathrm{ex}}$, and $\sigma_{\mathrm{T}}$ as function of $\mathrm{L}$ for the $\mathrm{m}=0.5$ and $3.0 \mathrm{~F}^{-1}$ cases of Figure 1 . Both the approximate and exact results are shown for $\sigma_{\text {ex }}$ and $\sigma_{\mathrm{T}}$. 


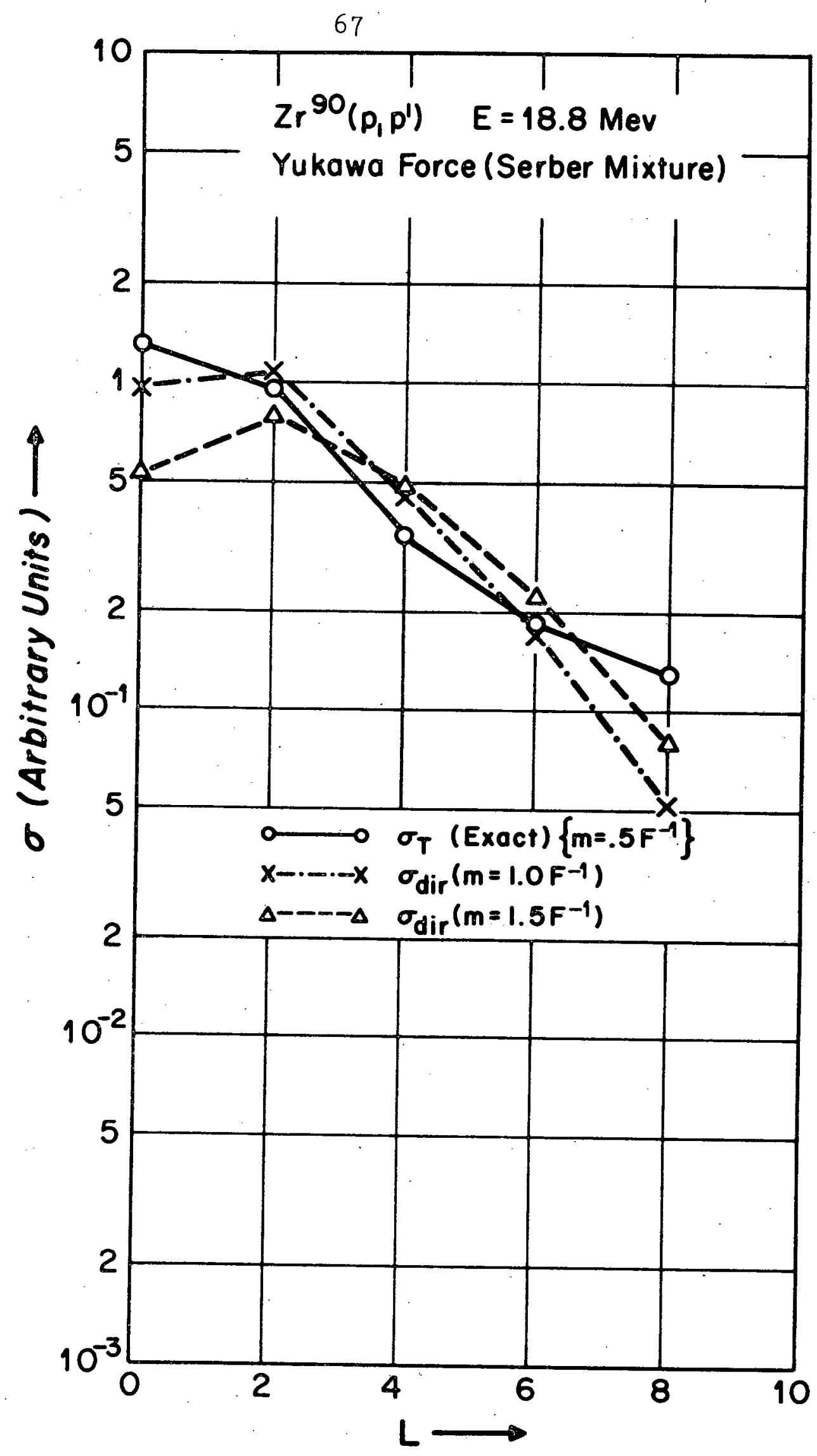

Figure 3.--Exact value of $\sigma_{\mathrm{T}}$ for a $2 \mathrm{~F}$ range Yukawa force is compared witn $\sigma$ dir for a $I F$ and $2 / 3 F$ range Yukawa force as a function of $L$. 
distributions fall off only slightly slower with angle. than do the direct and, for the lower multipoles, the latter exhibit quite a bit more structure. Both the direct and approximate exchange angular distributions for the .33F range Yukawa force are essentially the same as the approximate exchange angular distributions shown in Fig. 4 .

In Fig. 5 the $\mathrm{L}=2$ direct (D) and exact exchange (E) angular distributions given by a Yukawa force with a range slightly longer than $1 F$ are compared. 18 Comparing these with the $L=2$ results in Fig. 4 indicates that the approximate exchange angular distributions may fall off faster with angle than do the exact exchange angular distributions. This also might be multipole dependent, but no comparison is available for the higher multipoles. The differences, for large angles, between both the direct and exchange angular distributions shown in Fig. 5 and those which correspond in Fig. 4 is attributable to the fact that the spin-orbit term in the optical potential has been excluded in obtaining the results shown in Fig. 5. Inclusion of optical spin-orbit coupling is found to have no effect on the ratios of integrated cross sections discussed previously.

\section{Form Factors}

The direct and exchange form factors corresponding to the results given in Fig. 2 are shown in Fig. 6. The overall normalization for each force range is again arbitrary with the relative scaling given correctly. For the short range 


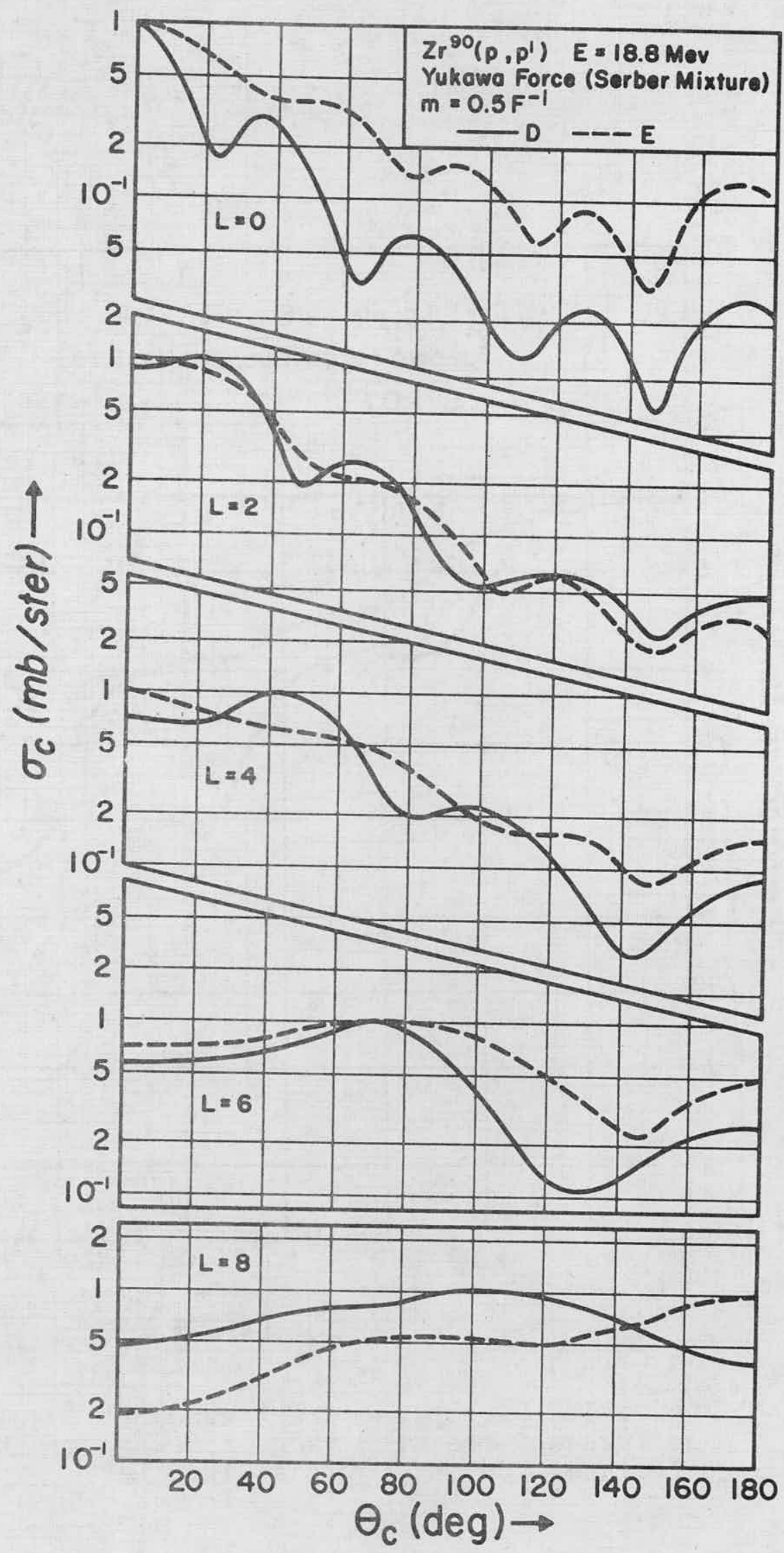

Figure 4.--Direct and approximate exchange angular distributions for $2 \mathrm{~F}$ range Yukawa force for $\mathrm{L}=0,2,4,6$, and 8 transitions in $\mathrm{Zr} 90+\mathrm{p}$ at $18.8 \mathrm{MeV}$. 


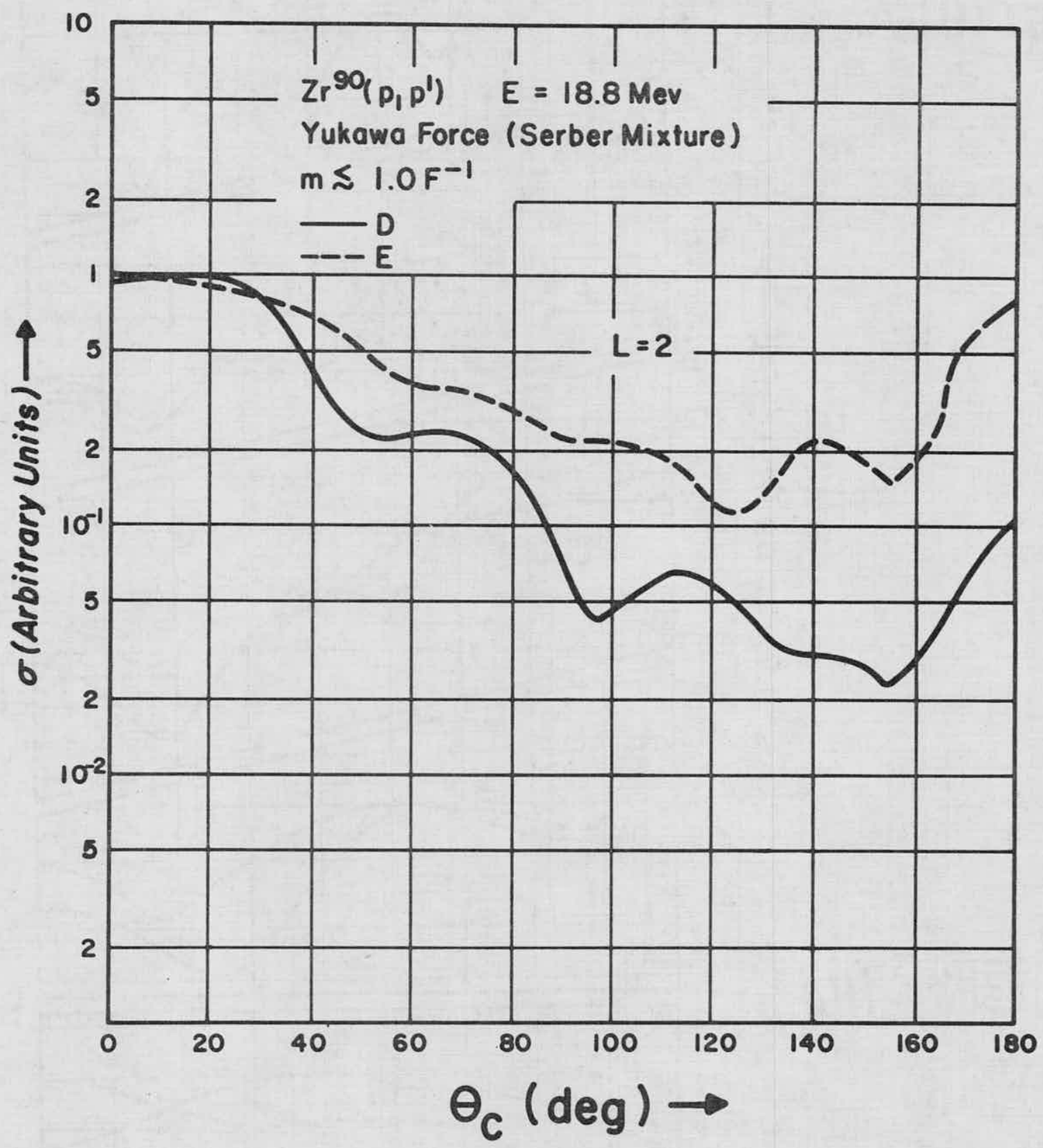

Figure 5.--Direct and exact exchange angular distribution for Yukawa force with range somewhat greater than $1 \mathrm{~F}$ for $\mathrm{L}=2$ transition in $\mathrm{Zr90}+\mathrm{p}$ at $18.8 \mathrm{MeV}$. 
force $\left(\mathrm{m}=3 . \mathrm{OF}^{-1}\right)$ all of the form factors including the exchange (zero range) form factor are similar in shape with the peak magnitude of the exchange form factor assuming a value intermediate to those for the $\mathrm{L}=0,2$ and $\mathrm{L}=4,6,8$ direct form factors. This ordering is preserved for the long range force $\left(m=.5 \mathrm{~F}^{-1}\right)$; however, the differences in peak magnitude of the direct form factors is much more pronounced. Here the direct form factors are also much broader than the exchange form factor and peak at larger radii. The differences in peak radii between the $\mathrm{L}=0,2,4,6$, and 8 direct form factors is not very large for either force range. Examination of these form factors emphasizes again that a multipole independent assumption has been made about the exchange scattering and that the variation of $\sigma_{\mathrm{ex}} / \sigma_{\mathrm{dir}}$ is due mostly to the changes in the direct scattering. The exact results call for additional multipole and energy dependence in the exchange scattering.

\section{Relation of Energy Dependence} to Interaction Form

It is found for both the long range and short range Yukawa interactions that the approximate $\sigma_{e x} / \sigma_{\text {dir }}$ ratios are given to within $5 \%$ by taking the square of the ratio of the areas under the appropriate form factor curves. The area is defined as the product of the peak magnitude and the half width of the curve. To what extent this will be true for other transition densities and forces with different radial forms is not known. For example, it has been noted that 


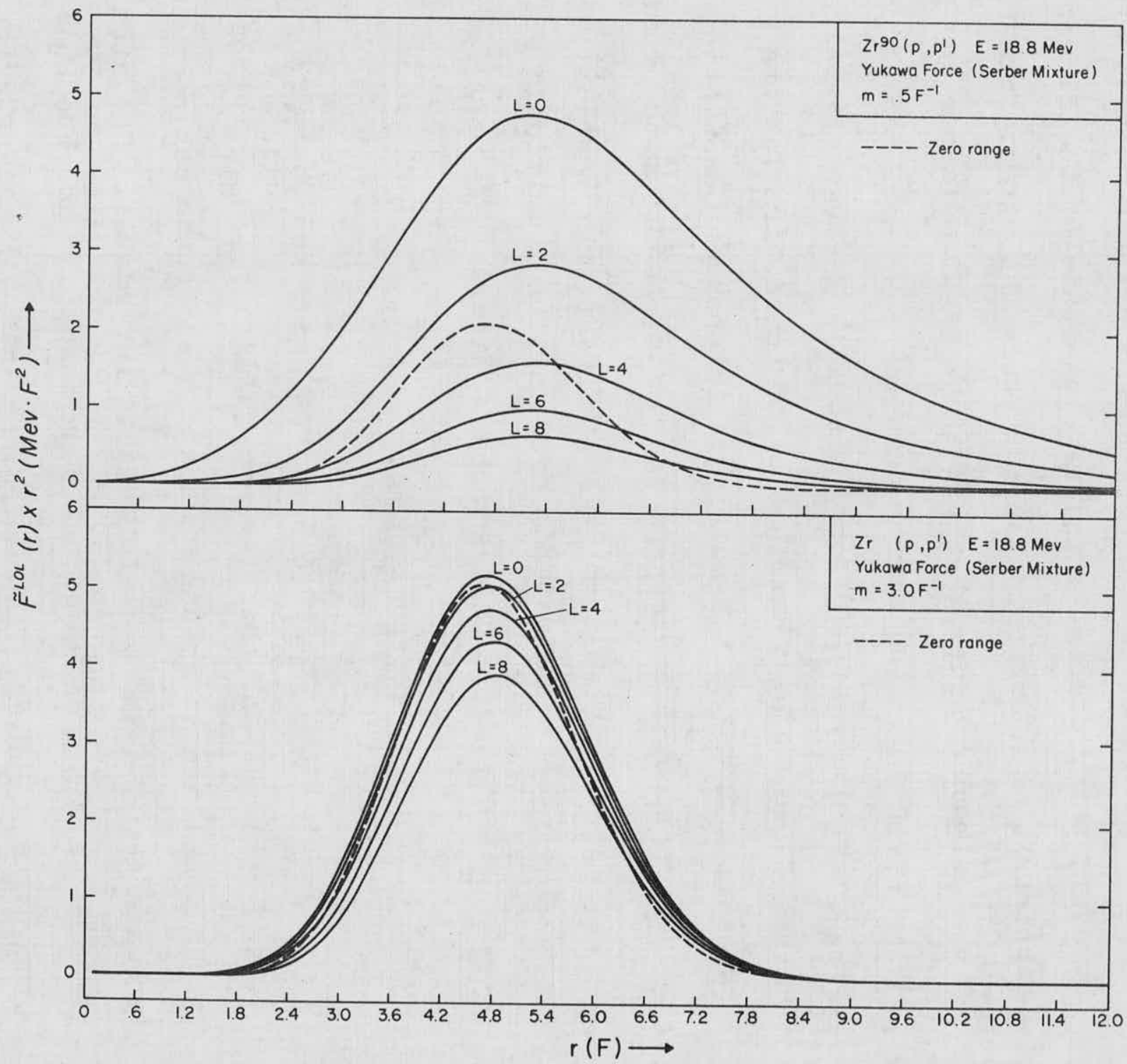

Figure 6.--Direct and exchange form factors corresponding to results of Figure 2 . 
$L=0,2,4,6$, and 8 form factors obtained from the $\left(\lg _{9 / 2}\right)^{2}$ transition density with a Gaussian interaction of $2 \mathrm{~F}$ range exhibit a much larger spread in peak positions than is seen for the $2 \mathrm{~F}$ range Yukawa force. ${ }^{9}$

Nonetheless, this observation indicates that the energy dependence of $\sigma_{\mathrm{dir}} / \sigma_{\text {ex }}$ for a given transition density and multipole can be written

$$
\frac{\sigma_{\operatorname{ex}}}{\sigma_{\operatorname{dir}}}(E)=\left[A^{(I)}(E)\right]^{2} K
$$

with $K$, the ratio of the integrated cross section obtained with a $\delta$-function force of unit strength to the direct integrated cross section, being roughly constant. Eq. (4) implies that

$$
\frac{\sigma_{\text {ex }}}{\sigma_{\operatorname{dir}}}\left(E_{1}\right) \approx\left[A^{(I)}\left(E_{1}\right) / A(I)\left(E_{2}\right)\right]^{2} \frac{\sigma_{\text {ex }}}{\sigma_{\operatorname{dir}}}\left(E_{2}\right) .
$$

Fig. 7 gives $\sigma_{\text {dir }}, \sigma_{\text {ex }}$, and $\sigma_{\mathrm{T}}$ as a function of energy for the $L=2$ transition and the $I F$ range Yukawa force. The conventions are the same as those for Fig. 2. This figure simply illustrates that $\sigma_{\mathrm{T}}$ drops off faster with energy than does $\sigma_{\text {dir }}$ due to the behavior of $\sigma_{\text {ex }}$, that the approximation is improving with increasing energy, and that large errors are not observed in $\sigma_{T}$ even at the lower energies. The result indicated by the center line and labeled $\sigma_{\text {ex }}$ (F.T.) has been obtained by fixing $K$ from the value of $\sigma_{\text {ex }} / \sigma_{\text {dir }}$ at $25 \mathrm{MeV}$ and then using Eq. (5) to get this ratio at the higher energies. 


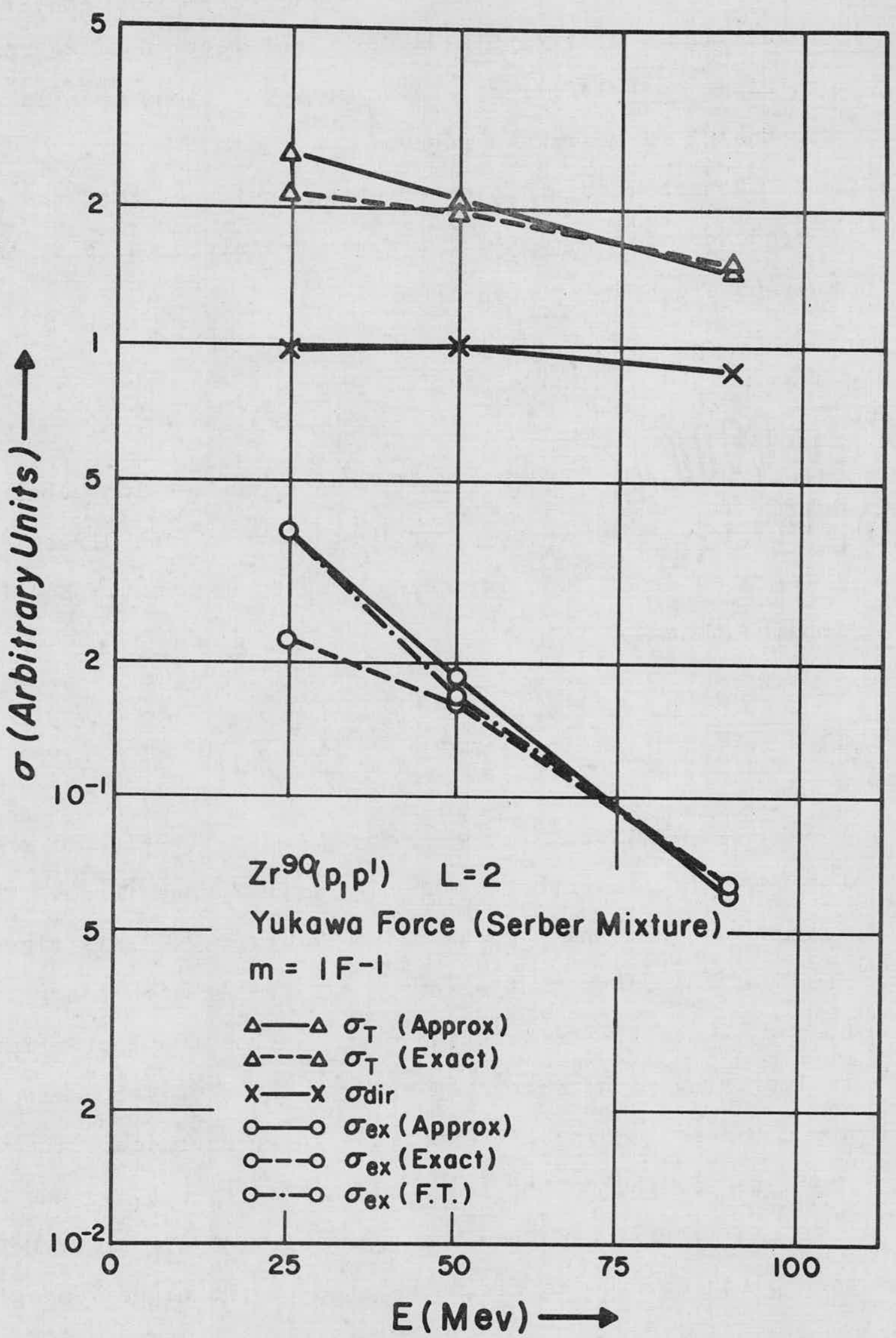

Figure 7.--Energy dependence of $\sigma_{\text {dir }}, \sigma_{\mathrm{ex}}$, and $\sigma_{\mathrm{T}}$ for $\mathrm{L}=2$ transition in $\mathrm{Zr} 90$ calculated withip range force as in Figure 1. Both approximate and exact results are given for $\sigma$ and the force Is also indicated. 
The value of $\sigma_{\text {ex }}$ is then easily extracted given $\sigma_{\text {dir }}$. The agreement between $\sigma_{e x}$ (Approx) and $\sigma_{e x}$ (F.T.) is quite good. ${ }^{\dagger}$ This relation can be used with Eq. (3) to estimate, for a particular transition and force, the ratio of the enhancement of the direct total cross section due to exchange at two different energies given the value of $\sigma_{e x} / \sigma_{d i r}$ at one energy. Table 3 gives the values of $\alpha(20) / \alpha(80)$ which have been obtained through the use of these relations for the $\mathrm{L}=0,2,4,6$, and 8 transitions in $\mathrm{Zr}^{90}$ for Yukawa forces with $\mathrm{m}=.5,1.0,1.5,2.0,2.5$, and $3.0 \mathrm{~F}^{-1}$. The values of $\sigma_{\mathrm{ex}} / \sigma_{\mathrm{dir}}$ at $20 \mathrm{MeV}$ are those shown in the lower graph of Fig. 1. The differences between these ratios for the different multiples increase with the force range. For the $2 \mathrm{~F}$ range force the energy dependence of $\sigma_{\mathrm{T}}$ should be quite different for the various multipoles provided the direct total cross sections vary slowly with energy, i.e. $\sigma_{\mathrm{T}}$ for the higher multipoles should fall off faster with energy than for the lower multipoles where $\sigma_{e x}$ is making a smaller contribution. This is an example of an effect due to antisymmetrization which might be used to study the properties of the projectiletarget interaction.

Non-Normal Transfers

The cross sections for the non-normal transfer specified by the triad $(J-l, I, J)$ have been calculated for these five transitions for a $I F$ range force. ${ }^{19}$ In all cases they were found to be smaller than the corresponding normal exchange

Tote that $\sigma_{e x}(E x a c t)$ and $\sigma_{e x}(F . T$.$) will only agree if the$ extrapolation is for energies above $40 \mathrm{MeV}$; however, reasonable approximate values of $\alpha$ (see Eq. (3I)) might be obtained over the entire energy region. 
TABLE 3.--Approximate energy dependence of enhancement of direct cross section due to exchange as a function of multipole and range of a Yukawa force.

\begin{tabular}{l|llcll}
\hline $\mathrm{m}\left[\mathrm{F}^{-1}\right]$ & $\mathrm{L}=0$ & $\mathrm{~L}=2$ & $\begin{array}{c}\alpha(20) / \alpha(80) \\
\mathrm{L}=4\end{array}$ & $\mathrm{~L}=6$ & $\mathrm{~L}=8$ \\
\hline 0.5 & 1.31 & 1.60 & 2.27 & 3.26 & 5.10 \\
1.0 & 1.56 & 1.74 & 2.07 & 2.47 & 3.22 \\
1.5 & 1.54 & 1.62 & 1.75 & 1.92 & 2.10 \\
2.0 & 1.43 & 1.47 & 1.52 & 1.60 & 1.67 \\
2.5 & 1.33 & 1.35 & 1.38 & 1.41 & 1.45 \\
3.0 & 1.30 & 1.26 & 1.27 & 1.29 & 1.32 \\
\hline
\end{tabular}

cross sections. Only for the $\mathrm{L}=8$ transition, where exchange scattering is dominant, was an appreciable contribution to $\sigma_{\mathrm{T}}$ obtained. Here the (718) component gave $25 \%$ of $\sigma_{\mathrm{T}}$. This is encouraging, however, it must be noted that the $S=0$ and $S=I$ components of the proton-proton force are equal in magnitude. If the component of the force contributing to the non-normal transfer was larger than that contributing to the normal transfer (as in the hypothetical situation outlined in Section 6 of Chapter 2) the non-normal transfer would clearly be more important for the $\mathrm{L}=8$ transition and might be important for the lower multipoles also.

3. Transitions in $\mathrm{C}^{12}, \mathrm{O}^{16}$, and $\mathrm{Ca}^{40}+\mathrm{p}$. As a further check, comparison calculations have been performed for some of the transitions which are being used 
In this work to "calibrate" the effective interaction. These are the excitation of the $1^{+} \mathrm{T}=1 \quad(Q=-15.1 \mathrm{MeV}), 2^{+} \mathrm{T}=0$ $(\mathrm{Q}=-4.43 \mathrm{MeV})$ and $3^{-} \mathrm{T}=0(\mathrm{Q}=-9.63 \mathrm{MeV})$ levels of $\mathrm{C}^{12}$ by 28.05 and $45.5 \mathrm{MeV}$ protons, the excitation of the $3^{-} \mathrm{T}=0 \quad(Q=-6.13 \mathrm{MeV})$ level of $0^{16}$ by $24.5 \mathrm{MeV}$ protons and the excitation of the $3^{-} \mathrm{T}=0 \quad(\mathrm{Q}=-3.73 \mathrm{MeV})$ and $5^{-\mathrm{T}}=0(\mathrm{Q}=-4.48 \mathrm{MeV})$ levels of $\mathrm{Ca}^{40}$ by 25 and $55 \mathrm{MeV}$ protons. The experimental results to be shown for $\mathrm{C}^{12}$ at $45.5 \mathrm{MeV}$ and for $\mathrm{Ca}^{40}$ at $55 \mathrm{MeV}$ have been published 72,45 while the results for $\mathrm{C}^{12}$ at $25 \mathrm{MeV}$ is the unpublished work of P. Locard and S. Austin. The experimental results for $\mathrm{O}^{16}$ at $24.5 \mathrm{MeV}$ and for $\mathrm{Ca}^{40}$ at $25 \mathrm{MeV}$ are the unpublished work of $W$. Benenson and C. Gruhn, respectively.

In these calculations the interaction was taken to be the 1 I range Yukawa "equivalent" to the $K-K$ force which was given in Chapter 4. The wave functions used in these calculations are specified in the following chapter. For the time being it is sufficient to say that both the exact and approximate results have been obtained in a consistent manner from these wave functions. Optical spin-orbit coupling has beem omitted and the optical parameters used are given in. the next chapter.

Only normal transfers have been considered. The targets being considered all have $\mathrm{O}^{+}$ground states, therefore the $\mathrm{J}$ transfer must equal the total angular momentum of the final state. All of the transitions except the one ending at the $I^{+} \mathrm{T}=1$ state of $\mathrm{C}^{12}$ are of normal parity. For these only the 
cross section specified by the triad $(J, 0, J)$ has been calculated while that specified by $(J-I, I, J)$ has been calculated for the abnormal parity transition.

The exact and approximate results are compared with each other and with experiment in Fig. 8,9, and 10. The total differential cross sections are shown in all cases. The dashed curves are the approximate results and the solid curves are the exact results. The direct differential cross sections and the approximate and exact exchange differential cross sections are shown only in Fig. 8 which gives the results for the $\mathrm{L}=3$ transition in $\mathrm{C}^{12}$. Here the direct angular distributions are shown as center lines and dashed and solid curves are used to designate the approximate and exact exchange angular distributions, respectively. No ambiguity results from not distinguishing the exchange and total differential cross sections in this figure as the latter are always larger. Not much need be said about the differences between the exact and approximate results. It is quite clear that no serious discrepancies have been introduced in treating exchange by this approximation. The differences that are observed are generally consistent with those noted in discussing the $\mathrm{Zr}^{90}$ results, $i . e$. the approximate total differential cross sections tend to overestimate the exact ones at the lower energies by less than $40 \%$ and the differences all but vanish at the high energies. The shapes are generally consistent with some deviations being noted at 


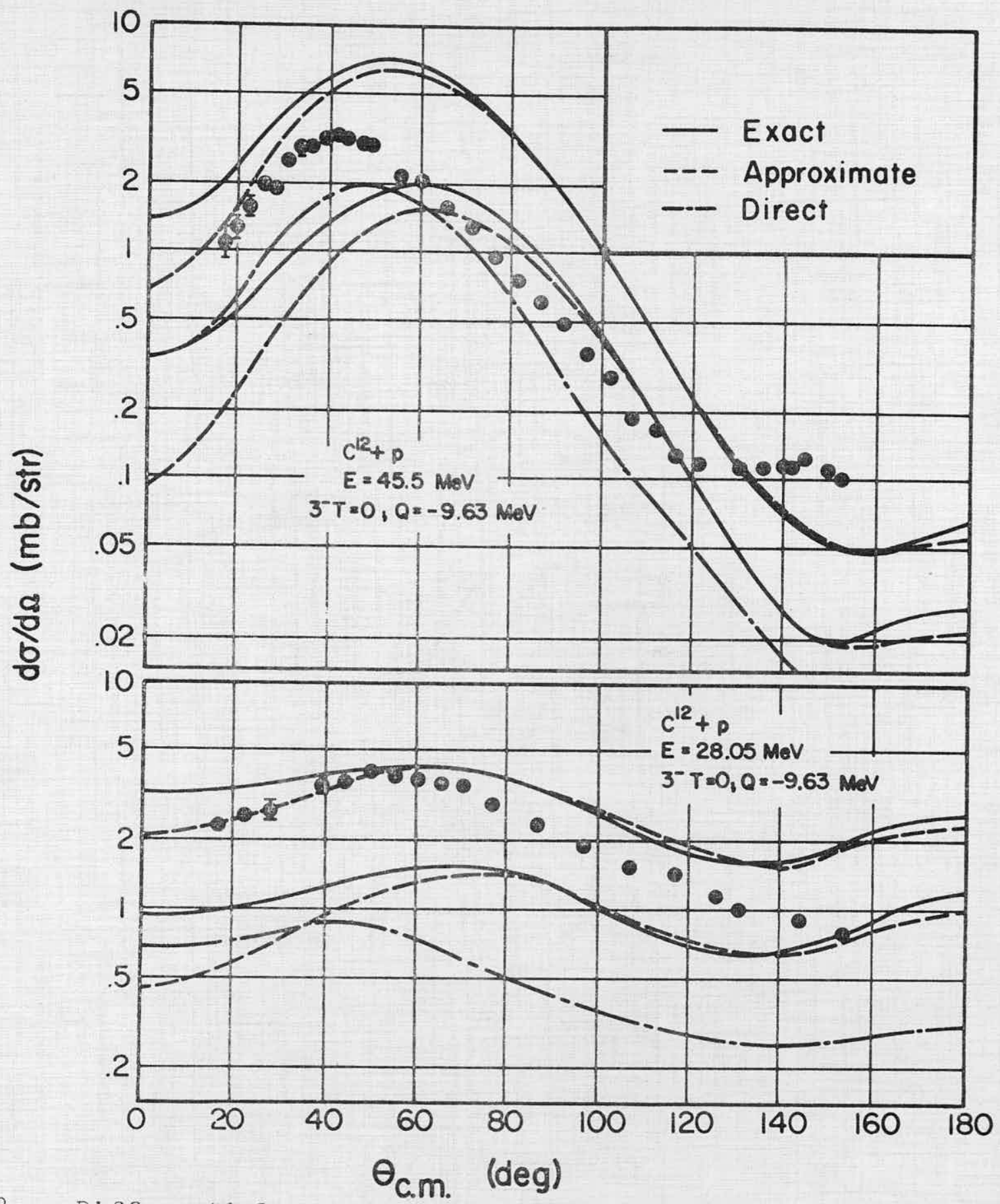

F'igure 8.-- Differential cross sections obtained with the K-K "equivalent" interaction for the $L=3$ transition in the $C^{12}\left(p, p^{\prime}\right)$ reaction at 28.05 and 45.5 MeV. A decomposition of the cross sections into djrect and exchange components is shown and a comparison of approximate and exact results is given. The direct component which is not affected by the approximation is shown as a center line. The lower of the two sets of exact and approximate results shown is the exchange component and the upper set is the total differential cross section. 


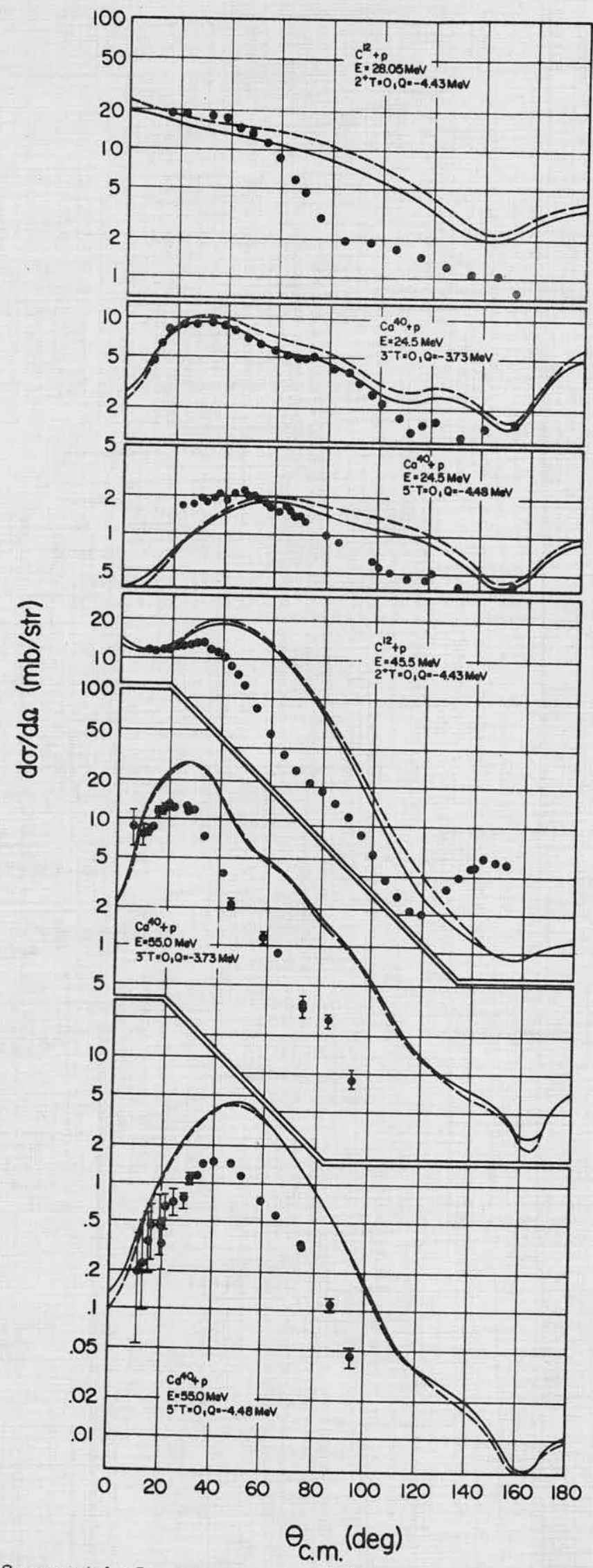

Figure 9.--Total differential cross sections obtained with the $K-K$ "equivalent" interaction, computed approximately and exactly, for the $\mathrm{L}=2$ transition in $\mathrm{Cl}$ at 28.05 and $45.5 \mathrm{MeV}$ and the $\mathrm{L}=3$ and $\mathrm{L}=5$ 


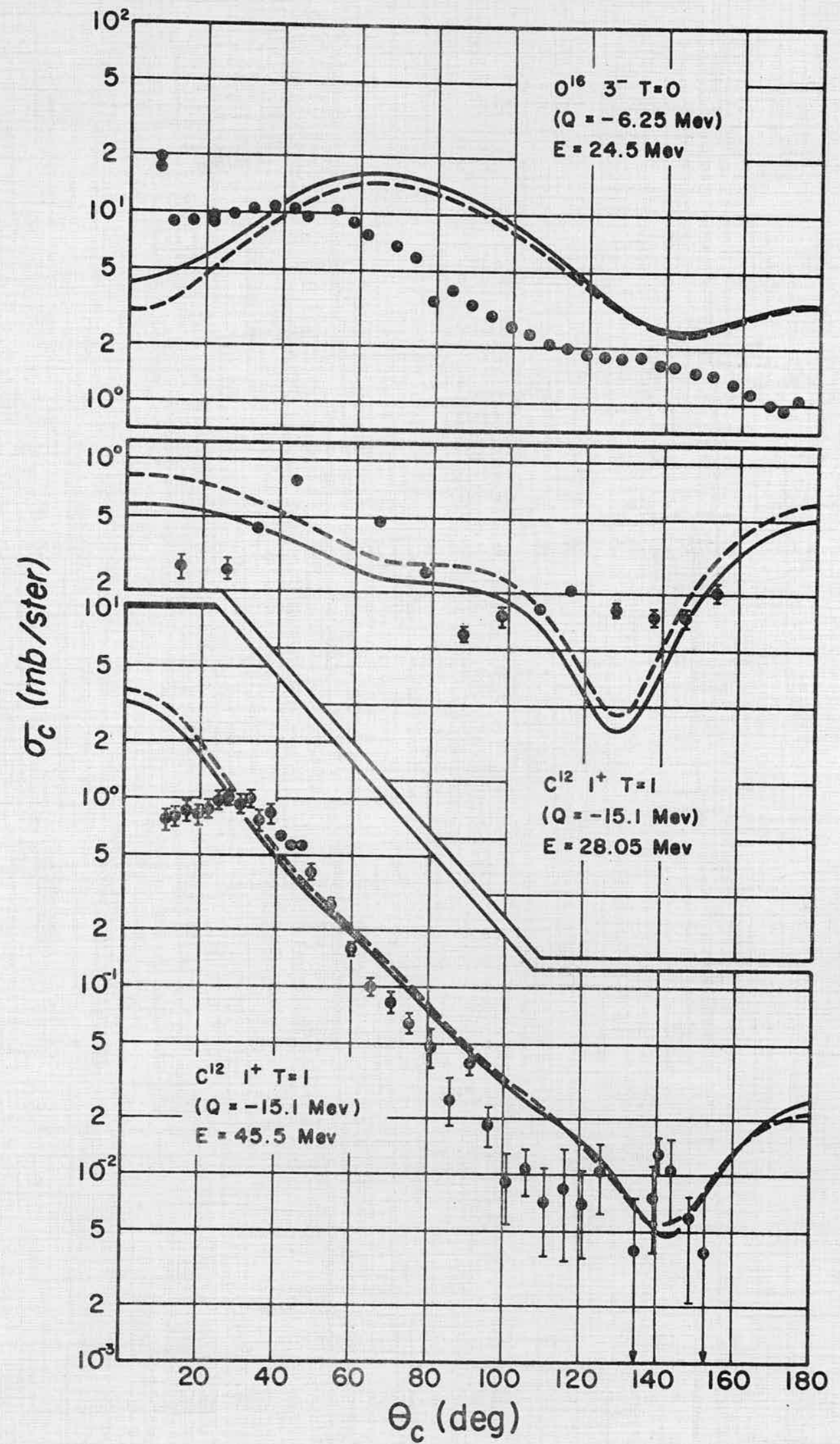

Figure 10.--Total differential cross sections obtained the $\mathrm{K}-\mathrm{K}$ "equivalent" interaction, computed approximately and exactly, for the excitation of the $3^{-T}=0(Q=-6.13 \mathrm{MeV})$ level of 016 at $24.5 \mathrm{MeV}$ and the excitation of the $I^{+} \mathrm{T}=1(\mathrm{Q}=-15.1)$ level of $\mathrm{C}^{12}$ at 28.05 and $45.5 \mathrm{MeV}$. 
forward angles for the $\mathrm{L}=3$ transitions in $\mathrm{C}^{12}$ and $\mathrm{O}^{16}$. The only peculiarity that is observed occurs for these same cases-here it is found that the approximate results tend to underestimate the exact results. In the light of the other results which have been presented this is not expected and an explanation is not readily available.

The value of $\sigma_{\text {dir }}$ and the approximate values of $\sigma_{\text {ex }}$ and $\sigma_{\mathrm{T}}$ for the $\mathrm{L}=3$ transition in $\mathrm{C}^{12}$ at $28.05 \mathrm{MeV}$ are 6.59 , 12.9 , and $36.5 \mathrm{mb}$, respectively. At $45.5 \mathrm{MeV}$ the values $7.48,7.46$, and $28.8 \mathrm{mb}$ are obtained. Note that $\sigma_{\text {dir }}$ has changed only slightly with energy and that $\sigma_{\text {ex }} / \sigma_{\text {dir }}$ $(28.05)=1.96$ and $\sigma_{\text {ex }} / \sigma_{\text {dir }}(45.5)=.997$ which are in the ratio 1.97 . The value one would predict using Eq. (5) and Table 1 is 1.86 .

The comparison of the results with experiment is of some interest. It is found that this $1 \mathrm{~F}$ range Yukawa force yields results which are in reasonable agreement with experiment at the lower energies but appreciably overestimate the higher energy data. ${ }^{\dagger}$ Thus it is concluded that the experimental data favor an interaction whose range is longer than IF.

4. Summary

Although the results which have been presented do not constitute a complete study of the approximation it is felt that they demonstrate that it is probably qualitatively

The optical parameters used in the calculation for the $L=3$ transition in 016 were not very good. Better parameters are given in Ref. 73. The results shown in Fig. 10 should be reduced by a factor of $2-3$. 
correct over the entire medium energy region and may be quantitatively valid at energies exceeding $40 \mathrm{MeV}$. For the lighter nuclei considered it was found that the shapes of the total differential cross sections computed approximately were in reasonable agreement with those computed exactly with the possible exception of the $\mathrm{L}=3$ transitions in $\mathrm{C}^{12}$ and $0^{16}$. Here differences are noted between the exact and approximate exchange angular distributions. Differences were also noted for the $\mathrm{L}=2$ transition in $\mathrm{Zr}^{90}$. The energy dependence of $\sigma_{e x}$ and $\sigma_{\mathrm{T}}$ has been related to that of $\sigma_{\text {dir }}$ through the Fourier transform of the force being used. Further, it would appear that the damping of exchange scattering in the nuclear interior, i.e. the correction terms discussed in Appendix A, would improve the approximate results. As the exact calculation of the exchange transition amplitude is quite involved it is felt that this approximation and the relations based on it can be put to good use in any analysis of the effects due to antisymmetrization.

5. $\mathrm{K}-\mathrm{K}$ Force

The singlet even and triplet even components of the $K-K$ force have the form

$$
\begin{array}{rlrl}
V(r) & =0 & r \leq d \\
& =V e^{-m r} & & r>d
\end{array}
$$

which lead to the following expression for the Fourier transform. 


$$
\begin{aligned}
V\left(\lambda^{2}\right)= & 4 \pi V e^{-m d}\left(m^{2}+\lambda^{2}\right)^{-2}\left\{\frac{\sin \lambda d}{\lambda}\left[\left(m^{2}+\lambda^{2}\right)(m \alpha-1)+2 m^{2}\right]\right. \\
& \left.+\cos \lambda d\left[d\left(m^{2}+\lambda^{2}\right)+2 m\right]\right\}
\end{aligned}
$$

As this force acts only in even states the $A(1)\left(\lambda^{2}\right)$ are given directly by the Fourier transform of the appropriate component of the interaction. Table 4 gives the strengths of all components of $A(1)$. The notation $A_{S E}$ and $A_{T E},{ }_{S T}$, and $A_{S}^{p p}$ and $A P$ is used. The last row in this table gives the 20 to $80 \mathrm{MeV}$ ratios as was done in Table 1.

The values of these ratios, as compared to those given for the Yukawa functions, are illustrative of the long range character of the $K-K$ interaction. The results of Section 3 of this chapter indicated that a long range force is needed. The behavior of the $A^{(I)}(E)$ is, for the most part, regular. The extreme long range behavior of $A_{10}$ and the fairly short range character of $A_{1}^{p n}$ indicate that a great deal of cancellation has taken place in constructing them. This leads one to suspect that these components of the interaction are not well determined.

Unlike interactions with regular functional forms, the long range behavior of the $\mathrm{K}-\mathrm{K}$ interaction is not reflected in its range parameter. It is attributable, instead, to the presence of the "hole" in the interaction. To see this it is only necessary to note that

$$
V\left(\lambda^{2}\right)=4 \pi \int_{0}^{\infty} j_{0}(\lambda r) V(r) r^{2} d r
$$


TABLE 4.--Strengths of components of exchange interaction for $K-K$ force as a function of the lab energy. Fixed separation distances are assumed.

\begin{tabular}{|c|c|c|c|c|c|c|c|c|c|}
\hline \multirow[t]{2}{*}{$E[\mathrm{MeV}]$} & \multicolumn{9}{|c|}{$A^{(I)}(E)\left[\operatorname{MeV} \cdot F^{3}\right]$} \\
\hline & $\mathrm{A}_{\mathrm{SE}}$ & $\mathrm{A}_{\mathrm{TE}}$ & $\mathrm{A}_{00}$ & $\mathrm{~A}_{10}$ & $A_{11}$ & $\mathrm{~A}_{0}^{\mathrm{PP}}$ & $\mathrm{A}_{1}^{\mathrm{pp}}$ & $A_{0}^{p n}$ & $A_{1}^{p n}$ \\
\hline 0 & -868 & -1200 & -388 & 87.8 & 129 & -217 & 217 & -558 & -41.5 \\
\hline 10 & -660 & -946 & -301 & 64.6 & 100 & -165 & 165 & -437 & -35.7 \\
\hline 20 & -500 & -745 & -234 & 47.2 & 77.8 & -125 & 125 & -342 & -30.7 \\
\hline 30 & -376 & -585 & -180 & 33.9 & 60.1 & -94.0 & 94.0 & -267 & -26.2 \\
\hline 40 & -278 & -457 & -138 & 23.7 & 45.9 & -69.6 & 69.6 & -206 & $-22 \cdot 3$ \\
\hline 50 & -201 & -352 & -104 & 15.7 & 34.6 & -50.3 & $50 \cdot 3$ & -157 & -18.9 \\
\hline 60 & -140 & -267 & -76.2 & 9.54 & 25.4 & -34.9 & 34.9 & -117 & -159 \\
\hline 70 & -90.6 & -196 & -53.8 & 4.72 & 17.9 & -22.7 & 22.7 & -850 & -13.2 \\
\hline 80 & -51.3 & -139 & -35.6 & .958 & 11.9 & -12.8 & 12.8 & -58.4 & -10.9 \\
\hline $0 / 80)$ & 9.75 & 5.36 & 6.57 & 49.4 & 6.57 & 9.75 & 9.75 & 5.87 & 2.82 \\
\hline
\end{tabular}


and to remember that the main envelope of the Bessel function appearing in this integral is confined to values of $r_{\sim} \frac{I}{\lambda}$. Since $\lambda_{20}$ and $\lambda_{80}$ are roughly $I$ and $2 \mathrm{~F}^{-1}$, respectively, while the cutoff radii are approximately $I F$, it is clear that this main envelope is falling within the "hole". Continuous exponential functions with the same range parameters as the singlet even and triplet even components of the $K-K$ force give $(20 / 80)$ ratios of 2.05 and 1.96 , respectively, as compared with the corresponding values of 9.75 and 5.36 given in Table 4.

It is interesting to estimate the effect of the energy dependence of the separation distances (which is being neglected in this work) on the values of $A^{(I)}$ (E) given in Table 4. To do this it is assumed that

$$
\begin{aligned}
& d_{s}=1.025+(.05 / 60)(E-20) \\
& d_{t}=0.925+(.03 / 60)(E-20)
\end{aligned}
$$

where $E$ is in $\mathrm{MeV}$. These linear relations represent reasonably well the energy dependence of the cutoff radii as calculated by Kallio and Kolltveit. 46 Table 5 contains the results obtained for $A^{(I)}$ (E) under this assumption. The values of $A^{(1)}$ (20) given in Table 5 are identical to those given in Table 4 as the separation distances have been fixed at this energy. It is seen that $\mathrm{A}_{\mathrm{SE}}$ is quite sensitive to this change while the effect on $A_{\mathrm{TE}}$ is smaller insofar as the $(20 / 80)$ ratios are concerned. The energy dependence of 
TABLE 5.--Same as Table 4 except separation distances vary with energy
according to Eq. (9.).

\begin{tabular}{|c|c|c|c|c|c|c|c|c|c|c|}
\hline \multirow[t]{2}{*}{$\mathrm{E}[\mathrm{MeV}]$} & \multirow[b]{2}{*}{$\mathrm{A}_{\mathrm{SE}}$} & \multirow[b]{2}{*}{$\mathrm{A}_{\mathrm{TE}}$} & \multicolumn{5}{|c|}{$A^{(1)}(E)\left[\mathrm{MeV} \cdot \mathrm{F}^{3}\right]$} & \multirow[b]{2}{*}{$\mathrm{A}_{1}^{\mathrm{pp}}$} & \multirow[b]{2}{*}{$A_{0}^{p n}$} & \multirow[b]{2}{*}{$\mathrm{A}_{1}^{\mathrm{pn}}$} \\
\hline & & & $A_{00}$ & $A_{10}$ & $\mathrm{~A}_{01}$ & $A_{11}$ & $A_{0}^{p p}$ & & & \\
\hline 0 & -884 & -121 & -393 & 90.0 & 172 & 131 & -221 & 221 & -566 & $-4 \cdot 12$ \\
\hline 10 & -667 & -952 & -304 & 65.6 & 137 & 101 & -167 & 167 & -440 & -35.6 \\
\hline 20 & -500 & -745 & -234 & 47.2 & 108 & 77.8 & -125 & 125 & -342 & -30.7 \\
\hline 30 & -370 & -580 & -178 & 33.1 & 85.6 & 59.3 & -92.4 & 92.4 & -264 & $-26 \cdot 3$ \\
\hline 40 & -267 & -446 & -134 & 22.2 & 67.0 & 44.6 & -66.8 & 66.8 & -201 & -22.4 \\
\hline 50 & -186 & -338 & -98.2 & 13.8 & 51.7 & 32.7 & -46.6 & 46.6 & -150 & -18.9 \\
\hline 60 & -122 & -249 & -69.6 & $7 \cdot 32$ & 39.1 & 23.2 & -30.5 & 30.5 & -109 & -15.9 \\
\hline 70 & -71.2 & -177 & -46.5 & $2 \cdot 32$ & 28.7 & 15.5 & -17.8 & 17.8 & -75.1 & $-13 \cdot 2$ \\
\hline 80 & -31.1 & -117 & -27.8 & -1.50 & 20.0 & 9.26 & $-7 \cdot 77$ & $7 \cdot 7.7$ & -47.8 & -10.8 \\
\hline$(20 / 80)$ & 16.1 & 6.37 & 8.41 & -31.6 & 5.42 & 8.41 & 16.10 & 16.1 & 7.16 & 2.86 \\
\hline
\end{tabular}


the separation distance is somewhat more pronounced in the former case. The differences between Tables 4 and 5 taken with the related effect on the direct component of the transition amplitude are large enough to produce noticeable differences in calculations; however, it is doubtful that they will be more important than the effects of the density dependence, imaginary component, and odd state components of the interaction which are also being neglected in this work. Further, most of the available data lies in the energy region from 20-50 MeV and none of the strong transitions observed are likely to isolate the singlet even component of the interaction where the effect is the largest.

6. Effective Range Forces

It has already been noted that the $(20 / 80)$ ratios for typical Yukawa forces are much smaller than most of those appearing in Table 4. In fact, since the long range limit of the Yukawa function is the Coulomb potential, the maximum value of $(20 / 80)$ for the Yukawa is 4. The Gaussian function, its Fourier transform, and the relation for the $(20 / 80)$ ratio are given below.

$$
\begin{gathered}
V(r)=V e^{-m^{2} r^{2}} \\
V\left(\lambda^{2}\right)=V \frac{\pi^{3 / 2}}{m^{3}} e^{-\lambda^{2} / 4 m^{2}} \\
V(20) / V(80)=\exp \left(\frac{\lambda^{2}-0^{-\lambda} 20}{4 m^{2}}\right)=e^{.731 / m^{2}}
\end{gathered}
$$


For an interaction of exponential form the corresponding relations are:

$$
\begin{aligned}
V(r) & =V e^{-m r} \\
V\left(\lambda^{2}\right) & =\frac{8 \pi V m}{\left(m^{2}+\lambda^{2}\right)^{2}} \\
V(20) / V(80) & =\frac{\left(m^{2}+\lambda_{80}^{2}\right)^{2}}{\left(m^{2}+\lambda_{20}^{2}\right)^{2}}
\end{aligned}
$$

Eq. (14) follows directly from Eq. (7) in the limit as d goes to zero. Note that $\mathrm{V}(20) / \mathrm{V}(80)$ for the Gaussian form can assume any value from $I$ to $\infty$ whereas $V(20) / V(80)$ for the exponential function can vary only from 1 to 16 .

The Fourier transforms of the components of the $K-K$ force clearly cannot be matched with a Yukawa function over the $0-80 \mathrm{MeV}$ energy range. It was found that this matching could not be achieved with an exponential function either. For example, an exponential function with $\mathrm{V}=-59.7 \mathrm{MeV}$ and $\mathrm{m}=.636 \mathrm{~F}^{-1}$ gives the same value for $(20 / 80)$ as the singlet even component of the $K-K$ force; however, it gives $V(0)=$ $-5840 \mathrm{MeV} . \mathrm{F}^{3}$ which is about six times the value given in Table 4 for the $K-K$ force $\left(A_{S E}^{(I)}(0)\right)$. In addition, $V(E)$ for $\mathrm{E}$ intermediate to 20 and $80 \mathrm{MeV}$ are smaller than corresponding values of $\mathrm{A}_{\mathrm{SE}}^{(\mathrm{I})}(\mathrm{E})$. A reasonably good match can be obtained with Gaussian functions. Gaussian interactions with V= $-34.9 \mathrm{MeV}$ and $\mathrm{m}=.567 \mathrm{~F}^{-1}$ and $\mathrm{V}=-67.3 \mathrm{MeV}$ and $\mathrm{m}=.660$ give the same $(20 / 80)$ ratios as the singlet even and triplet 
even components of the $K-K$ force, respectively. They also give $V(0)=-1070$ and $-1300 \mathrm{MeV} \cdot \mathrm{F}^{3}$ which are reasonable agreement with the corresponding $\mathrm{K}-\mathrm{K}$ force volume ingetrals. Table 6 contains the pertinent data for Gaussian, exponential, and Yukawa forces which fit the scattering lengths and effective ranges which are sufficient to characterize low energy nucleon-nucleon scattering 48,49 . Fig. Il shows the Fourier transforms of these forces compared with that for the $K-K$ force. The transforms for the $K-K$ force are bowed slightly upwards on the graphs, while those for the Gaussian are straight lines. Both the exponential and Yukawa transforms are bowed downwards. From the figure it is concluded that the Gaussian effective range force is quite similar to the $K-K$ force and that the Yukawa effective range force shows the greatest deviation from it. This is consistent with the remarks made in the preceding paragraph. In fact the strengths and ranges for the Gaussian functions given in Table 6 are nearly the same as those obtained by matching to the $\mathrm{K}-\mathrm{K}$ force.

These conclusions are not surprising. Like the effective range forces, the $K-K$ force is consistent with the low energy nucleon-nucleon scattering data. It is evident from Fig. Il that all of the forces are similar (on the average) for small values of $\mathrm{E}(<20 \mathrm{MeV})$. The Gaussian function has properties similar to the $K-K$ force and when the two are forced to correspond over a small region $(0-20 \mathrm{MeV})$ they 
automatically are similar over a much wider region $(0-80 \mathrm{MeV})$. On the other hand two dissimilar functions forced to correspond over a small region will not correspond over a wider interval.

TABLE 6.--Forces which are consistent with low energy nucleonnucleon scattering data.

\begin{tabular}{|c|c|c|c|}
\hline & & Singlet Even & Triplet Even \\
\hline Gaussian & $\begin{array}{l}\mathrm{V}(\mathrm{MeV}) \\
\mathrm{m}\left(\mathrm{F}^{-1}\right) \\
\mathrm{V}(0)\left(\mathrm{MeV} \cdot \mathrm{F}^{3}\right) \\
\mathrm{V}(20) / \mathrm{V}(80)\end{array}$ & $\begin{array}{r}-39.5 \\
.637 \\
-850 \\
6.06\end{array}$ & $\begin{array}{r}-71.0 \\
.676 \\
-1279 \\
4.95\end{array}$ \\
\hline Exponential & $\begin{array}{l}\mathrm{V}(\mathrm{MeV}) \\
\mathrm{m}\left(\mathrm{F}^{-1}\right) \\
\mathrm{V}(0)\left(\mathrm{MeV} \cdot \mathrm{F}^{3}\right) \\
\mathrm{V}(20) / \mathrm{V}(80)\end{array}$ & $\begin{array}{r}-138 \\
1.58 \\
-880 \\
3.39 \\
\end{array}$ & $\begin{array}{r}-186 \\
1.48 \\
-1442 \\
3.71 \\
\end{array}$ \\
\hline Yukawa & $\begin{array}{l}\mathrm{V}(\mathrm{MeV}) \\
\mathrm{m}\left(\mathrm{F}^{-1}\right) \\
\mathrm{V}(0)\left(\mathrm{MeV} \cdot \mathrm{F}^{3}\right) \\
\mathrm{V}(20) / \mathrm{V}(80)\end{array}$ & $\begin{array}{r}-47.6 \\
.855 \\
-957 \\
2.72\end{array}$ & $\begin{array}{r}-41.5 \\
.633 \\
-2060 \\
3.14\end{array}$ \\
\hline
\end{tabular}

The Yukawa effective range force has been selected for calculations in order to see how sensitive inelastic nucleonnucleus scattering is to the differences noted in Fig. Il. In using this force it is assumed that there is no interaction 


\section{FOURIER TRANSFORMS OF $K-K$ AND EFFECTIVE RANGE FORCES}

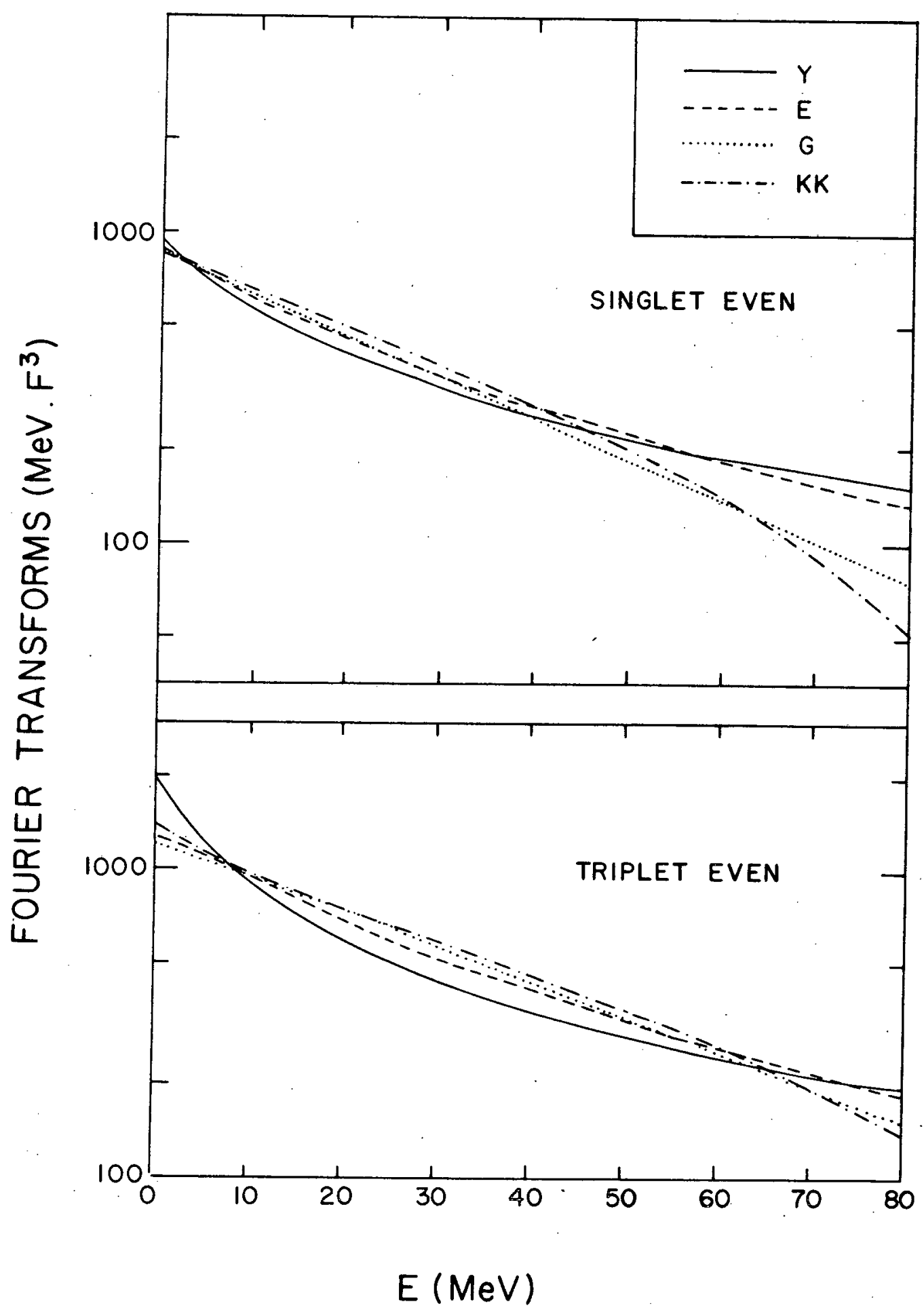

Figure 1l.--Comparison of Fourier transform of singlet even and triplet even components of the $K-K$ force with those of Gaussian(G)exponential, $(E)$ and Yukawa(Y) effective range forces. 
in odd states as is done for the $K-K$ force. Table 7 gives the values of $A^{(I)}(E)$ for the components of this force. The format is the same as that of Table 4 for the $K-K$ force. Note added in proof: A set of calculations from the O.R.N.L. group have just been made available through a preprint. ${ }^{74}$ These are for the $\mathrm{Zr}^{90}\left(\mathrm{p}, \mathrm{p}^{\prime}\right) \mathrm{Zr}^{90 *}$ reaction at $18.8 \mathrm{MeV}$ and $61.4 \mathrm{MeV}$ and for the $\mathrm{Zr}^{92}\left(\mathrm{p}, \mathrm{p}^{\prime}\right) \mathrm{Zr}^{92 *}$ reaction at $19.4 \mathrm{MeV}$. Again the $\mathrm{L}=0,2,4,6$, and 8 transitions in $\mathrm{Zr}^{90}$ have been considered and $\left(\lg _{9 / 2}\right)^{2}$ wave functions have been used. In $\mathrm{Zr}^{92}$ the $\mathrm{L}=0,2$, and 4 transitions corresponding to the $\left(2 d_{5 / 2}\right)^{2}$ neutron configuration have been treated. The long range part of the Hamada-Johnston (H-J) potential, including the second order tensor contributions to the triplet-even interaction, has been used for the projectile-target interaction. The odd state components of this interaction have been neglected and a separation distance of $1.05 \mathrm{~F}$ was used. In these calculations the exchange component of the D.W.A. transition amplitude has been treated exactly and $\sigma_{e x} / \sigma_{\alpha i r}$ ratios have been given.

These calculations have been repeated using the $\mathrm{K}-\mathrm{K}$ force and the approximate treatment of exchange of this work. The results are compared in Fig. l'. This comparison is reasonable because of the similarity of $\mathrm{K}-\mathrm{K}$ force and the $\mathrm{H}-\mathrm{J}$ interaction used above as was pointed out in Chapter 4. The discrepancies between the exact and approximate results shown in Fig. ' '' are much larger than any noted in the comparisons made in Chapter 5. For $L=0$ in $2 r^{90}$ at $18.8 \mathrm{MeV}$ the 
TABLE 7.--Strengths of components of exchange interaction for Yukawa effective range force as function of the lab energy

\begin{tabular}{|c|c|c|c|c|c|c|c|c|c|c|}
\hline \multirow{2}{*}{$\mathrm{E}[\mathrm{MeV}]$} & \multirow[b]{2}{*}{$\mathrm{A}_{\mathrm{SE}}$} & \multirow[b]{2}{*}{$\mathrm{A}_{\mathrm{TE}}$} & \multirow[b]{2}{*}{${ }^{A} 00$} & \multicolumn{4}{|c|}{$\mathrm{A}^{(1)}(\mathrm{E})\left[\mathrm{MeV} \cdot \mathrm{F}^{3}\right]$} & \multirow[b]{2}{*}{$\mathrm{A}_{1}^{\mathrm{pp}}$} & \multirow[b]{2}{*}{$\mathrm{A}_{0}^{\mathrm{pn}}$} & \multirow[b]{2}{*}{$A^{p n}$} \\
\hline & & & & $\mathrm{A}_{10}$ & ${ }^{A} \mathrm{OI}$ & $\mathrm{A}_{11}$ & $A_{0}^{p p}$ & & & \\
\hline 0 & -957 & -2060 & -565 & 50.9 & 326 & 188 & -239 & 239 & -891 & -137 \\
\hline 10 & -574 & -927 & -281 & 49.7 & 138 & 93.8 & -143 & 143 & -419 & -441 \\
\hline 20 & -410 & -598 & -189 & 39.4 & 86.5 & 63.0 & -102 & 102 & -275 & $-23 \cdot 5$ \\
\hline 30 & -318 & -441 & -142 & 32.1 & 62.9 & $47 \cdot 5$ & -79.6 & 79.6 & -205 & -154 \\
\hline 40 & -261 & -350 & -114 & 27.0 & $49 \cdot 3$ & 38.2 & -65.1 & 65.1 & -164 & $-11 \cdot 2$ \\
\hline 50 & -220 & -290 & -95.7 & 23.2 & 40.6 & 31.9 & $-55 \cdot 1$ & 55.1 & -136 & -8.66 \\
\hline 60 & -191 & -247 & $-82 \cdot 2$ & 20.4 & 34.4 & 27.4 & -47.8 & 47.8 & -117 & -703 \\
\hline 70 & -169 & -216 & -72.0 & 18.1 & 29.9 & 24.0 & -42.1 & 42.1 & -102 & -5.89 \\
\hline 80 & -151 & -191 & -64.1 & 16.3 & 26.4 & 21.4 & $-37 \cdot 7$ & 37.7 & $-90 \cdot 5$ & -5.05 \\
\hline$(20 / 80)$ & 2.72 & 3.14 & 2.95 & 2.42 & 3.28 & 2.95 & 2.72 & 2.72 & 3.04 & 4.65 \\
\hline
\end{tabular}




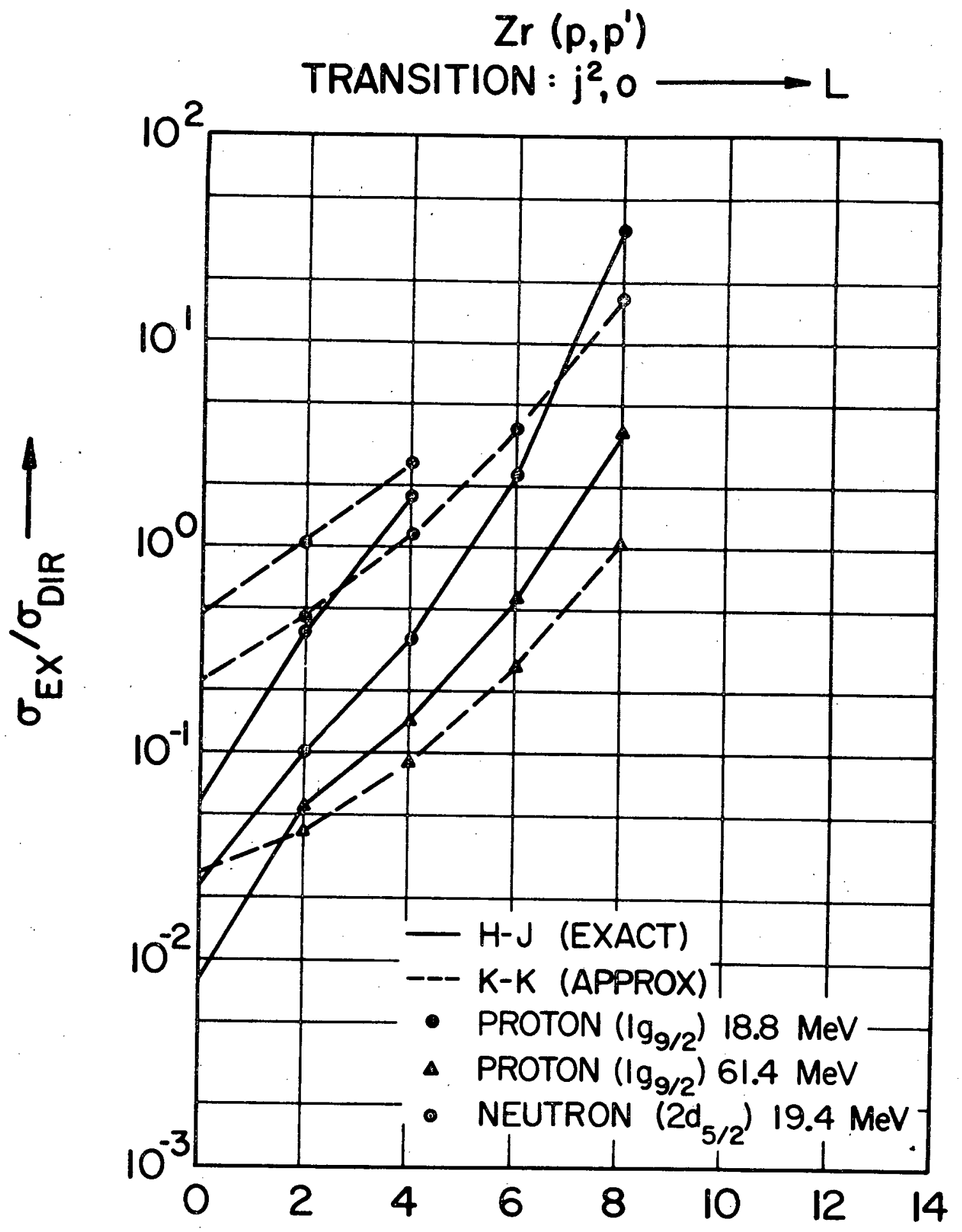

Figure I'.--Comparison of exact results obtained with long range part of $\mathrm{H}-J$ potential with approximate results given by $\mathrm{K}-\mathrm{K}$ force. 
approximate value of $\sigma_{\text {ex }} / \sigma_{\text {dir }}$ for the $K-K$ force is about 10 times the exact value obtained for the H-J force. For $L=2$ the approximate value is 4.7 times the exact value and for $L=8$ the approximate value falls about a factor of 2 below the exact value. Uncertainties this large might amount to factors of $1.5-3$ in the magnitude of the complete cross sections. Also note that the results indicate that the approximation is over-estimating the energy dependence due to exchange.

They have also reported that the Gaussian effective range force gives $\sigma_{e x} / \sigma_{d i r}$ ratios which are in agreement with those obtained with the long range part of the H-J potential. It is also estimated from their results that the H-J force gives somewhat weaker $(25 \%)$ cross sections than the $\mathrm{K}-\mathrm{K}$ force. Similar differences between the $\mathrm{H}-\mathrm{J}$ and $\mathrm{K}-\mathrm{K}$ forces were noted by Slanina in his optical potential calculations. 31,32 
CHAPTER 6

STUDY OF INTERACTION MODELS

IN D.W.A. CALCULATIONS

\begin{abstract}
As a matter of convenience this chapter is divided into two sections, i.e. Section A and section B. The D.W.A. results obtained with the impulse approximation pseudopotential, the $\mathrm{K}-\mathrm{K}$ force, and the Yukawa effective range force for select transitions in $\mathrm{C}^{12}$ and $\mathrm{Ca}^{40}$ are presented in Section A. Section B is devoted to a random collection of results. Some $\left(e, e^{\prime}\right)$ results are presented and occasional reference is made to $\left(p, p^{\prime}\right)$ studies at energies in excess of $100 \mathrm{MeV}$. The (e,e') results (at least electric multipole transitions) test only the proton activity in the transitions. This is sufficient, at least for $N=Z$ nuclei where protons and neutrons play symmetric roles. In viewing the results to be presented, keep in mind that a detailed fit to the experimental data is not the point of these calculations. An investigation of the interaction models with respect to the gross features of the experimental data is all that has been attempted.
\end{abstract}


1. Section A

The transitions considered here are the $\mathrm{L}=0,2$, and 3 transitions in $\mathrm{C}^{12}$ and the $\mathrm{L}=3$ and 5 transitions in $\mathrm{Ca}^{40}$ which were introduced in Section 3 of Chapter. $5 .^{+}$The $\mathrm{L}=0$ transition in $\mathrm{C}^{12}$ is an abnormal parity $\mathrm{T}=1$ transition. It tests the $t_{11}$ components of the. interactions. The other four transitions are normal parity $\mathrm{T}=0$ transitions which test the $t_{00}$ components of the interactions. Some of the results of Section $B$ provide information concerning other components of the projectile-target interaction.

Fig. I displays the R.P.A. vector ${ }^{50}$ for the $1^{+} \mathrm{T}=1$ $(Q=-15.1 \mathrm{MeV})$ state in $\mathrm{C}^{12}$. The analytic expressions for the $\mathrm{L}=0$ and $\mathrm{L}=2$ transition densities (Section 1 of Appendix B) are given along with graphs of these functions. The harmonic oscillator constant is also specified, i.e. $\alpha=.610 \mathrm{~F}^{-1}$ a value consistent with elastic electron scattering. 32,55 The calculation of the transition densities from the R.P.A. vectors is discussed in Section 3 of Appendix B. Note that $\mathrm{F}^{01 \mathrm{l}, \mathrm{l}}(\mathrm{r})$ is considerably larger than $\mathrm{F}^{2 l l, l}(\mathrm{r})$ and that both peak somewhat inside the nuclear surface. Only the $(011,1)$ triad is considered in the $\left(p, p^{\prime}\right)$ calculations of this work.

'See discussion of Fig. 8-10 in Chapter 5. 


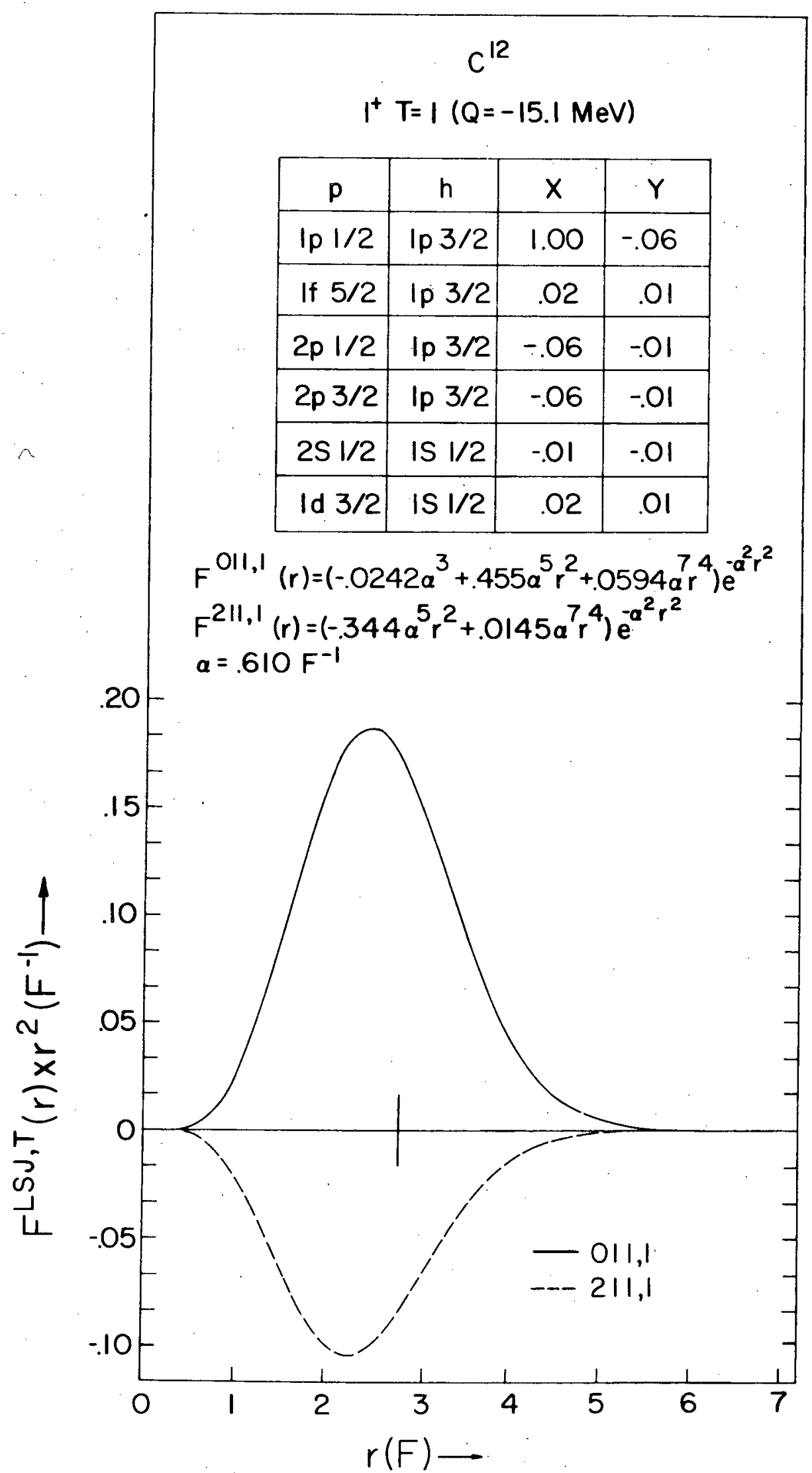

Figure 1.--R.P.A. vector and transition densities for $I^{+} T=I(Q=-15 . I \mathrm{MeV})$ level of $\mathrm{Cl}$. 
The phases of $\mathrm{X}_{j_{p} j_{h}}^{J T}$ and $\mathrm{Y}_{j_{p} j_{h}}^{J T}$ appearing in Fig. 1 differ from those given in Ref. 50 by the factor $(-1)^{j_{h}+1 / 2}$. The reason for this phase adjustment has been given elsewhere. $41-43$ It is made for all the R.P.A. vectors which are taken from Ref. 50-53 for use in this work. From Fig. I it is clear that the R.P.A. is saying that the $I^{+} T=1$ state in $\mathrm{C}^{12}$ is very nearly a pure $l p_{1 / 2}-1 p_{3 / 2}$ particle-hole pair. It is well. known that such a wave function predicts an electromagnetic MI form factor which ị much larger than experiment. 56 Investigation of this level, via the impulse approximation, in the $\left(p, p^{\prime}\right)$ reaction at $156 \mathrm{MeV}$ showed that reducing the $L=0$ transition density by $(3.3)^{1 / 2}$ is sufficient to produce a theoretical result which is in reasonable agreement with experiment. ${ }^{42,43}$ Such a factor is consistent with the electromagnetic studies. The expression for $F^{011,1}(r)$ and the graph of this function in Fig. 1 already contain this reduction factor.

Fig. 2-5 contain information, corresponding to that of Fig. I, for the four remaining, normal parity excitations in $\mathrm{C}^{12}$ and $\mathrm{Ca}^{40}$. The R.P.A. vectors shown have been taken from Ref. 50, 50, 54, and 51, respectively. These normal parity vectors exhibit considerably more mixing than the abnormal parity vector of Fig. 1. Examination of the size of the Y-amplitudes indicates that the effect of ground state correlations is much more important for normal parity transitions than for abnormal parity transitions. Here 


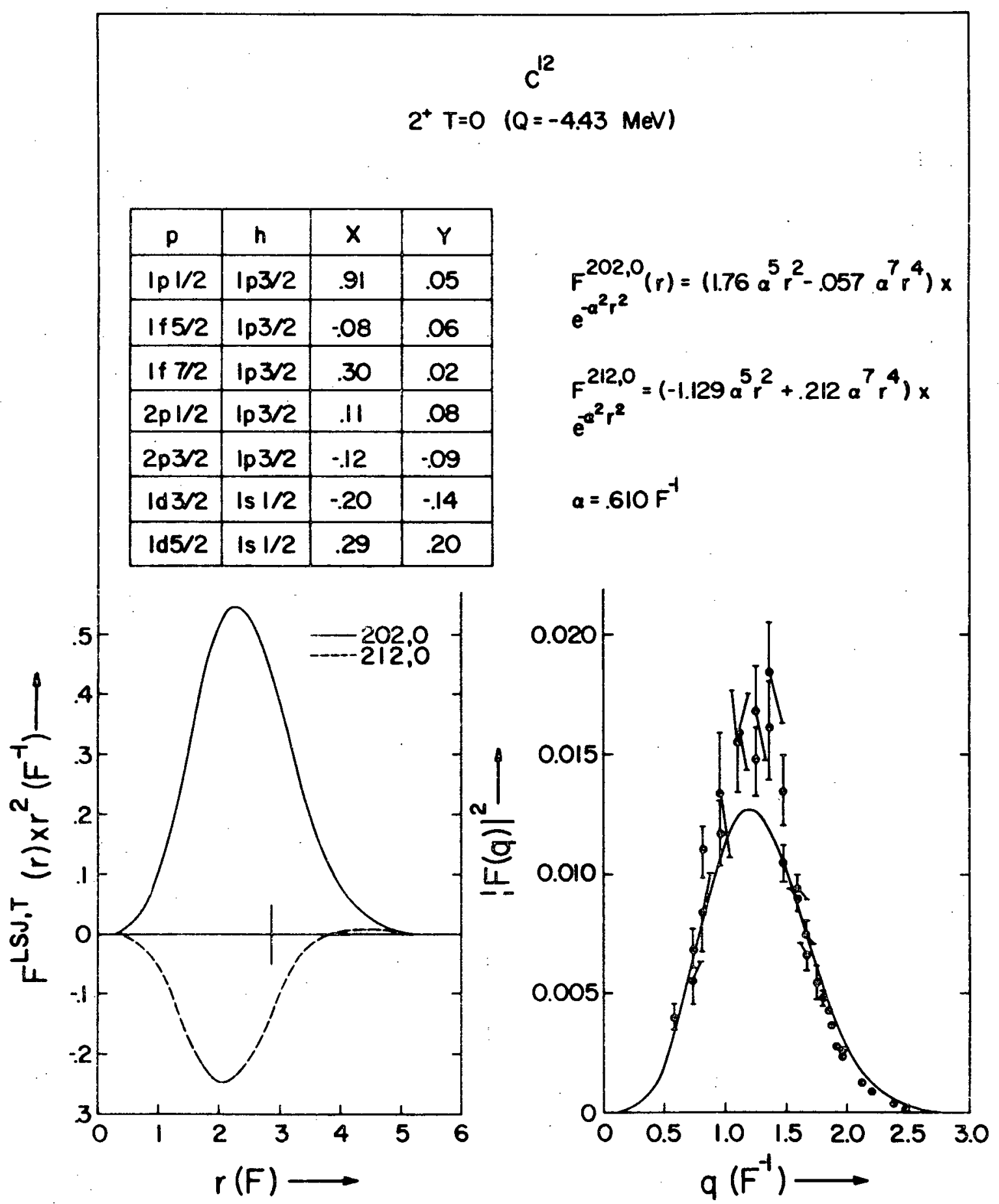

I'igure 2.--R.P.A. vector, transition densities, with theoretical and experimental inelastic electron scattering form factors for the $2^{+} \mathrm{T}=0(\mathrm{Q}=-4.43 \mathrm{MeV})$ level of $\mathrm{C}^{\mathrm{l} 2}$. 


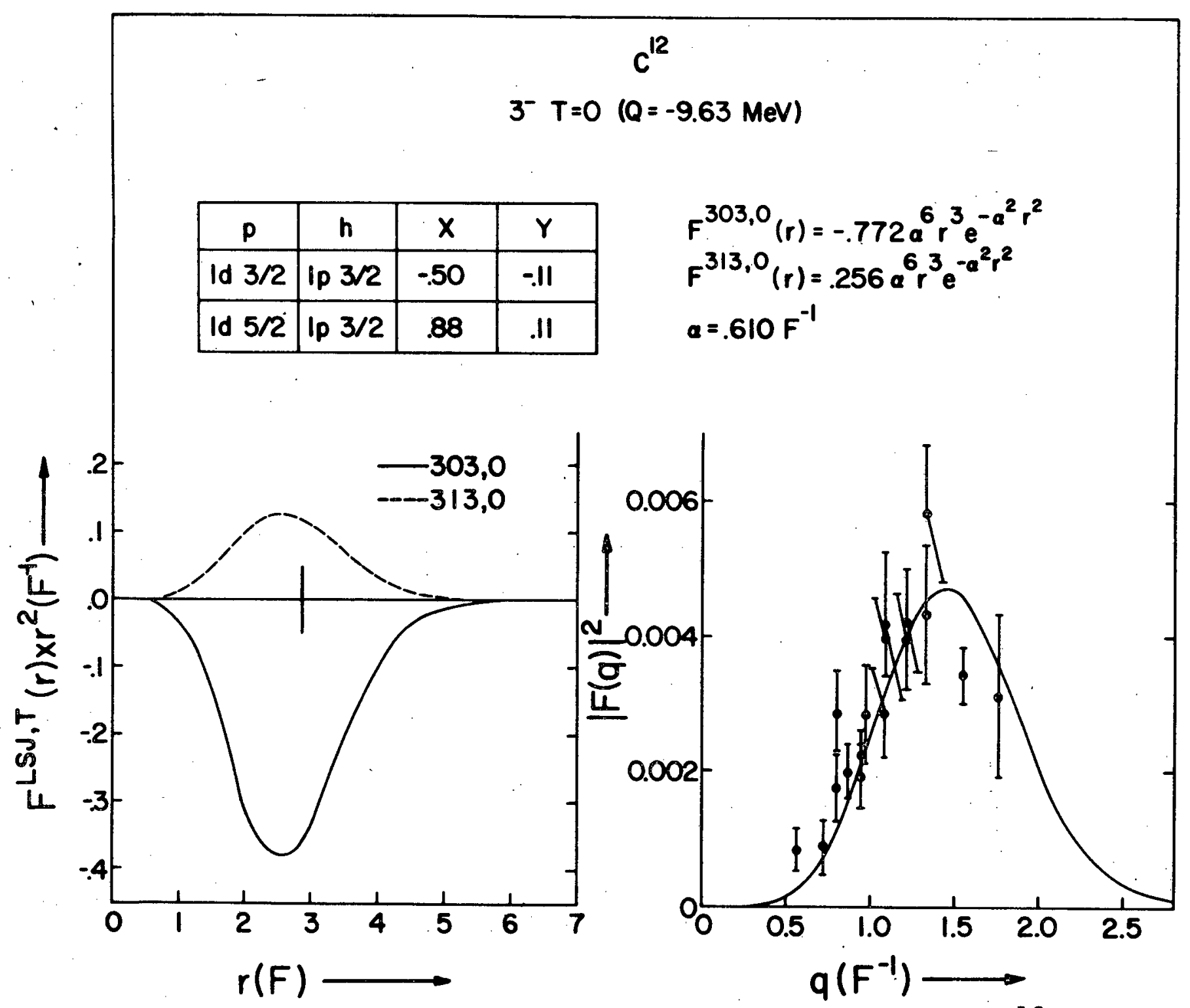

Figure 3.--Same as Figure 2. for $3^{-} \mathrm{T}=0(\mathrm{Q}=-9.63 \mathrm{MeV})$ level of $\mathrm{C}^{12}$. 


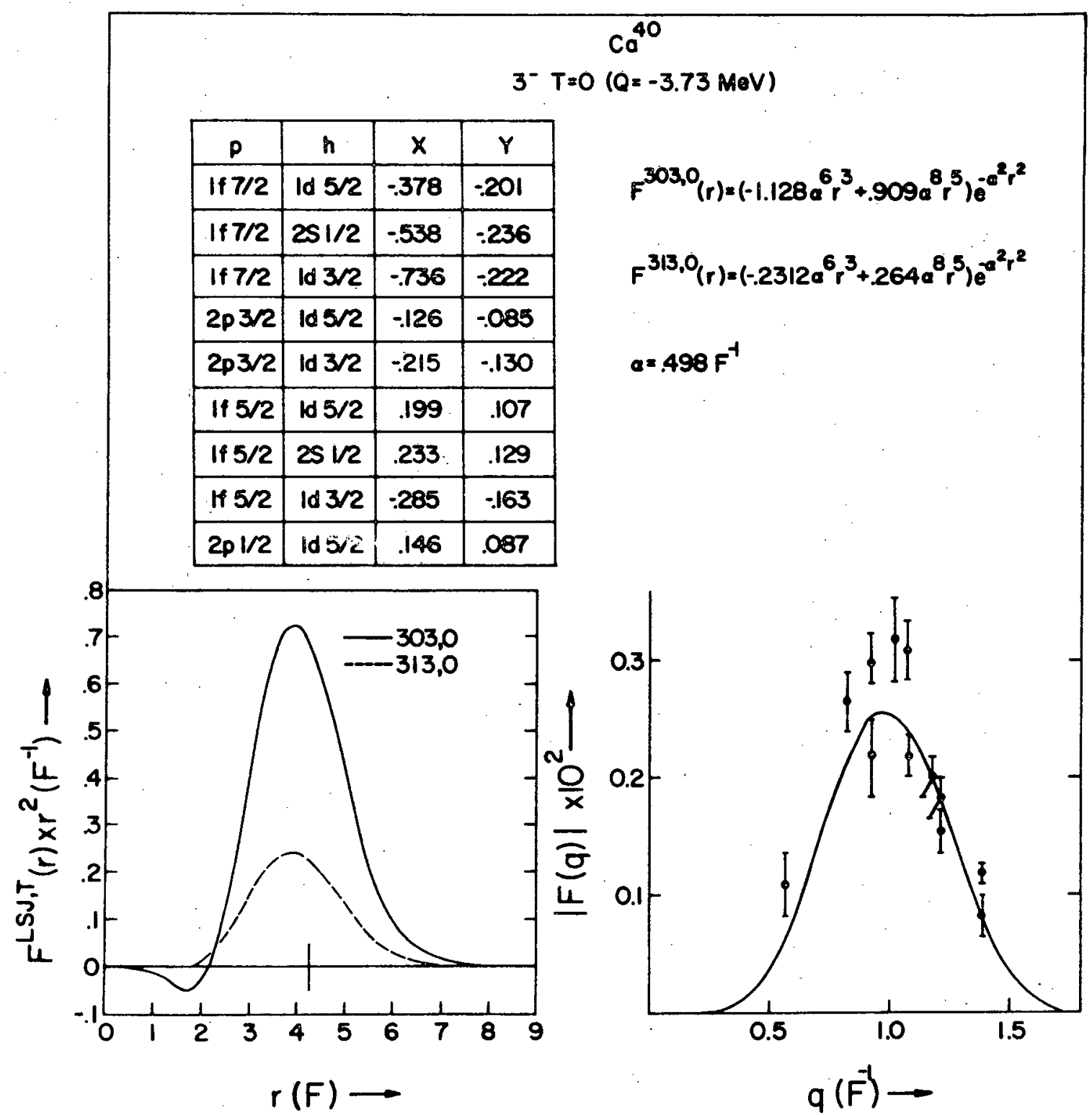

Figure 4.--Same as Figure 2 for $3^{-} \mathrm{T}=0(\mathrm{Q}=-3.73 \mathrm{MeV})$ level of $\mathrm{Ca}^{40}$. 


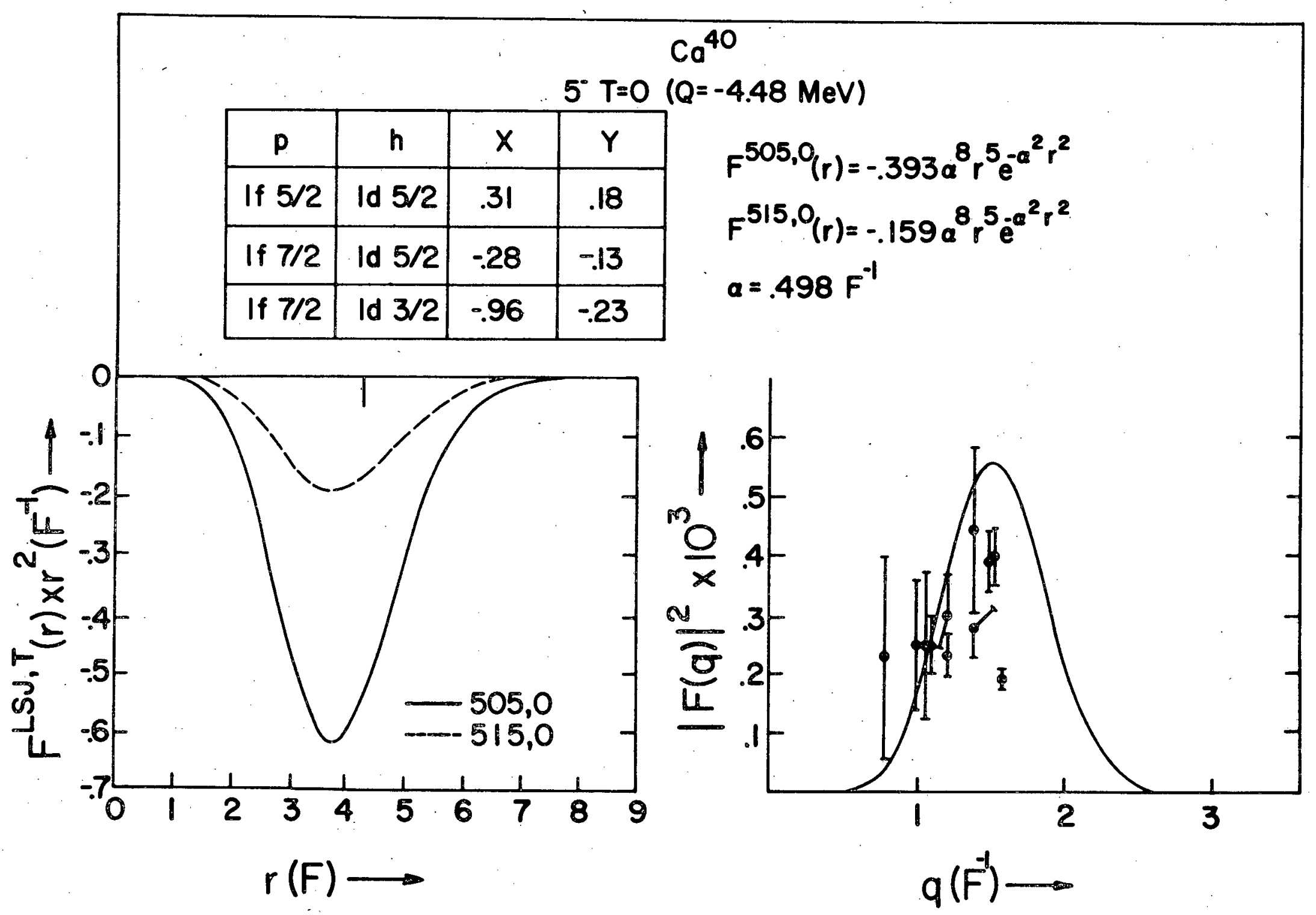

Figure 5.--Same as Figure 2 for $5^{-T}=0(Q=-4.48 \mathrm{MeV})$ level of $\mathrm{Ca}^{40}$. 
again it is seen that the transition densities peak inside the nuclear surface and that the $\mathrm{F}^{\mathrm{J} I J, 0}(r)$ transition densities can be neglected. The harmonic oscillator constant for $\mathrm{Ca}^{40}$ is taken from Ref. 32 .

Also contained in these figures is a comparison of the theoretical and experimental inelastic electron scattering form factors for the excitation of these levels. The calculation of the theoretical (e,e') form factors from the transition densities is discussed in Appendix $C$. These results are essentially the same as those contained in Ref. 55. They have been recalculated more as a check than for any other reason and are shown icr completeness. The overall agreement between theory and experiment is quite good, although the data for $\mathrm{Ca}^{40}$ is admittedly sparse. The ground state correlations are responsible for factors $1.5-3$ in the theoretical form factors which are from four to an order of magnitude larger than results obtained in single particle-hole excitation models. 55 The enhancement effects are largest for the $L=3$ transition in $\mathrm{Ca}^{40}$. The mixing in the R.P.A. vector. for this transition is evident from Fig. 4.

Looking at these results a bit more closely, it is seen that the $\mathrm{L}=2$ transitions for $\mathrm{C}^{12}$ gives a result for $|F(q)|^{2}$ which is about $15-20 \%$ too small. The theoretical form factor for the $\mathrm{L}=3$ transition in $\mathrm{C}^{12}$ has about the right magnitude, but it peaks at slightly too large a value of $q$. Ref. 55 extends the comparison of theory and experiment for these two transitions up to about $q=3.5 F^{-1}$. The theoretical results overestimate the data in this region 
which could be an indication that the theoretical transition densities are too large in the interior of the nucleus. The experimental data for the $\mathrm{L}=3$ transition in $\mathrm{Ca}^{40}$ is ambiguous and it is seen that the theoretical result in Fig. 4 falls in between the two sets of data points which are in disagreement. The corresponding result of Ref. 55 has been obtained with the R.P.A. vector given in Ref. 51 and it is in agreement with the upper set of data points in Fig. 4. The experimental data for the $L=5$ transition is also not very definitive. It appears that the theoretical result here could be a little too large and might peak at much too large a value of $q$.

It is concluded from this discussion that (1) the transition densities presented in Fig. 1-5 should not be responsible for any gross discrepancies in the ( $\left.p, p^{\prime}\right)$ results which are to be shown and (2) the effects of long range correlations, which are included in the R.P.A. vectors, are playing an important part in building up the magnitude of these transition densities. Better transition densities have been constructed for the $\mathrm{L}=2$ and $\mathrm{L}=3$ transitions in $\mathrm{C}^{12}$ by fitting directly to the experimental (e,e') data. ${ }^{75}$ These have not been used as they do not differ, a great deal from those presented here and the differences are well within the uncertainties associated with the local reduction of the D.W.A. transition amplitude. The (e,e') data for $\mathrm{Ca}^{40}$ is not sufficiently accurate and complete to even allow. consideration of improving the transition densities. 
D.W.A. calculations have been performed for $\mathrm{C}^{12}\left(\mathrm{p}, \mathrm{p}^{\prime}\right)$ $\mathrm{C}^{12^{*}}$ at 28.05 and $45.5 \mathrm{MeV}$ and $\mathrm{Ca}^{40}\left(\mathrm{p}, \mathrm{p}^{\prime}\right) \mathrm{Ca}^{40 *}$ at 25 and $55 \mathrm{MeV}$. The differential cross sections obtained for the above transitions using the impulse approximation pseudopotential are compared with experiment in Fig. 6-8. Corresponding results for the $K-K$ force are shown in Fig. 9-1I and those for the Yukawa effective range force are given in Fig. 12-14. The total differential cross sections for the $\mathrm{L}=3$ transition in $\mathrm{C}^{12}$ are decomposed into direct and exchange components in Fig. 10 ( $\mathrm{K}-\mathrm{K}$ force) and Fig. 13 (Yukawa effective range force). Optical parameters used in the calculations are given and referenced in Table $i$. The form used for this potential is given in Eq. (B.i3). A tabulation of the theoretical total integrated cross sections, $\sigma_{\mathrm{T}}$, is contained in Table 2. Values of $\sigma_{\text {dir }}$ and $\sigma_{\text {ex }}$ for the $K-K$ force and Yukawa effective range force are also displayed in this table.

A quick glance at Fig. 6-14 shows that all of these forces are giving a fair reproduction of the data. For the normal parity transitions it is found that the results obtained with the impulse approximation pseudo-potential and the $\mathrm{K}-\mathrm{K}$ force best reproduce the data. The impulse approximation gives results which are slightly. smaller in magnitude than the $K-K$ force. These differences are no larger than $20 \%$. The results for the Yukawa effective range force are found to underestimate these cross sections at the lower energies, but at the higher energies they are very close to the results 


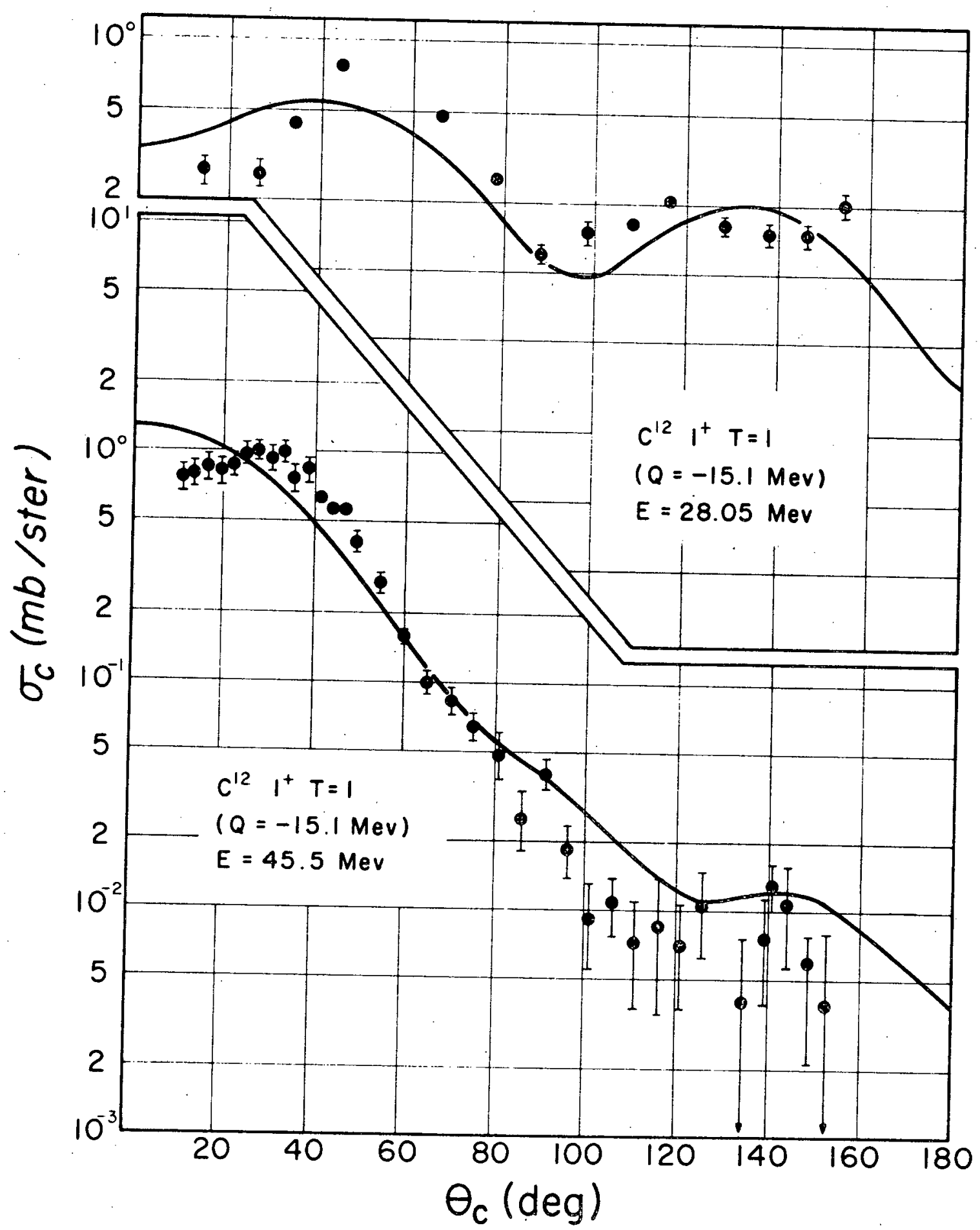

Figure 6.-Differential cross sections for $L=0$ transition in $\mathrm{C}^{12}$. The theoretical results have been obtained with the impulse approximation pseudo-potential. 


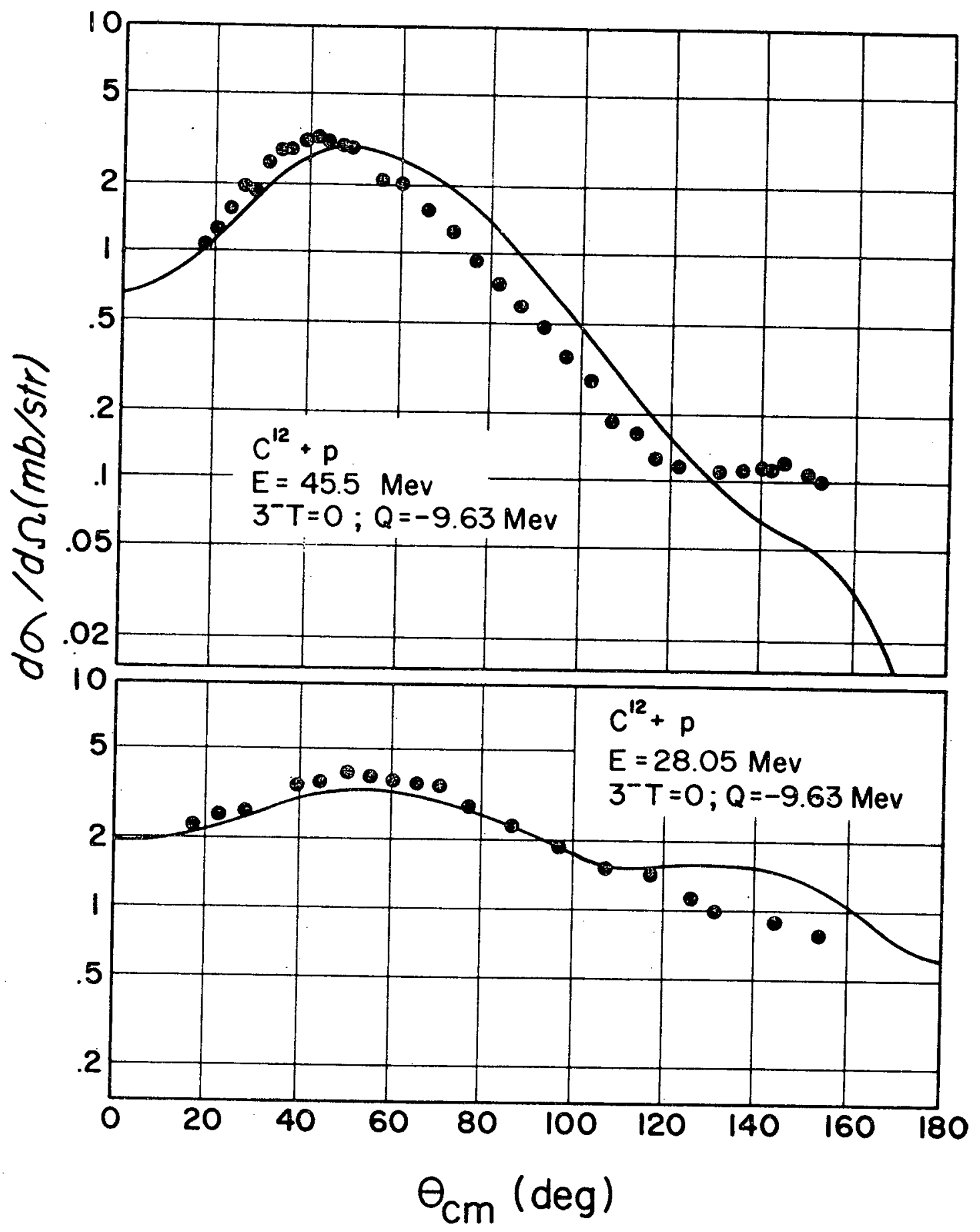

Figure 7.--Differential cross sections for $L=3$ transition in $\mathrm{C}^{12}$. The theoretical results have been obtained with the impulse approximation pseuod-potential. 


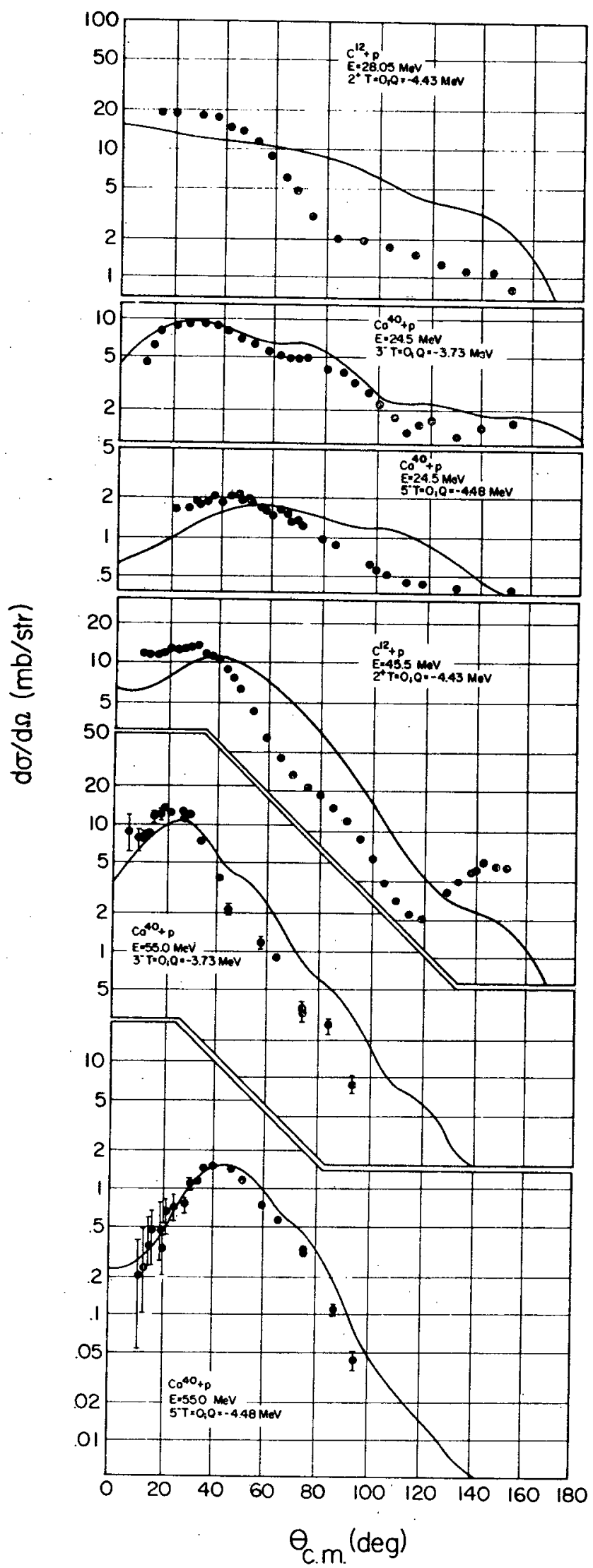

Figure 8.--Differential cross sections for $L=2$ transition in $\mathrm{C}^{12}$ and the $\mathrm{L}=3$ and $\mathrm{L}=5$ transitions in $\mathrm{Ca}^{40}$. results have been obtained with the impul. The theoretical pseudo-potential. obtained with the impulse approximation 


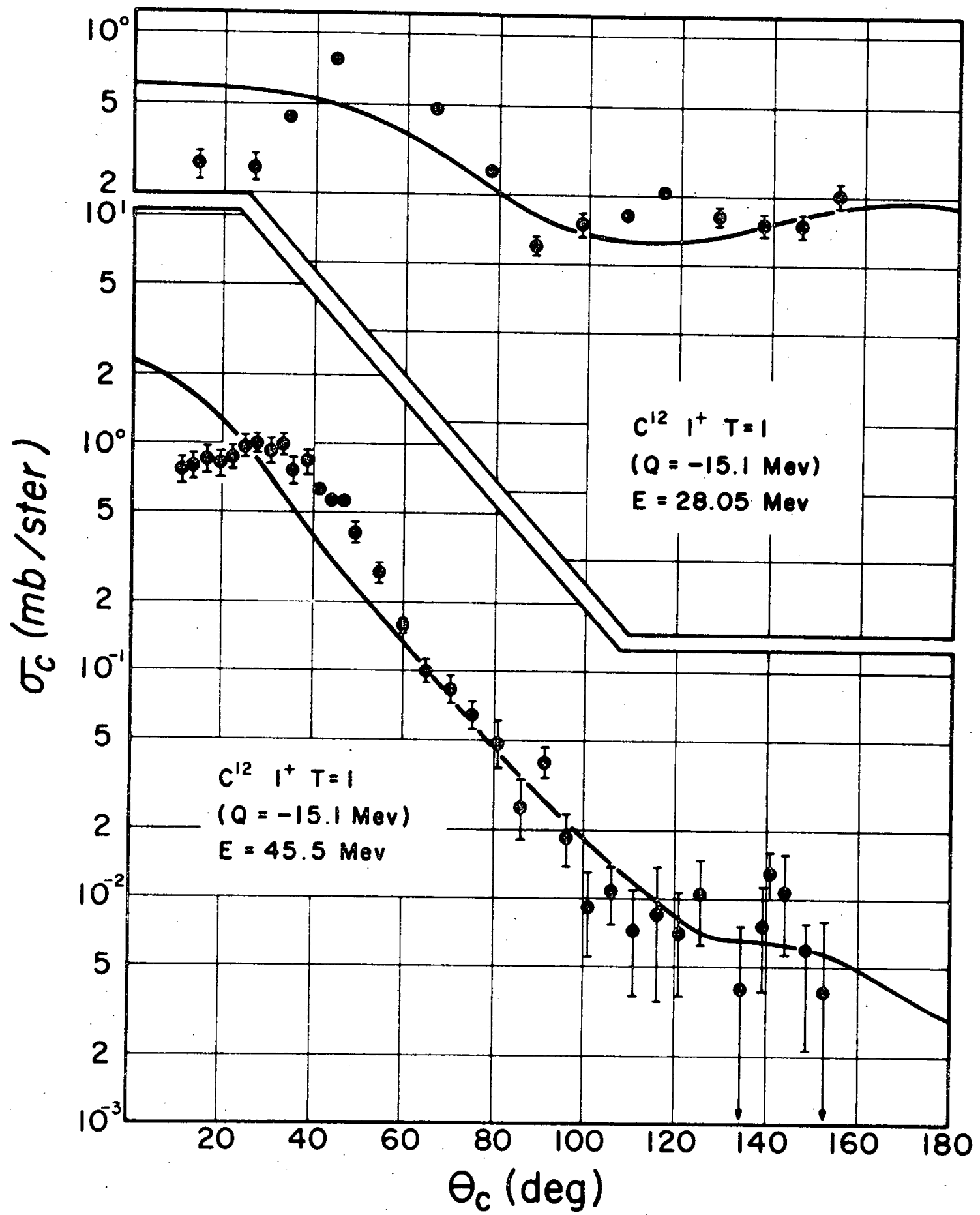

Figure 9.--Same as Figure 6 for K-K Force. 


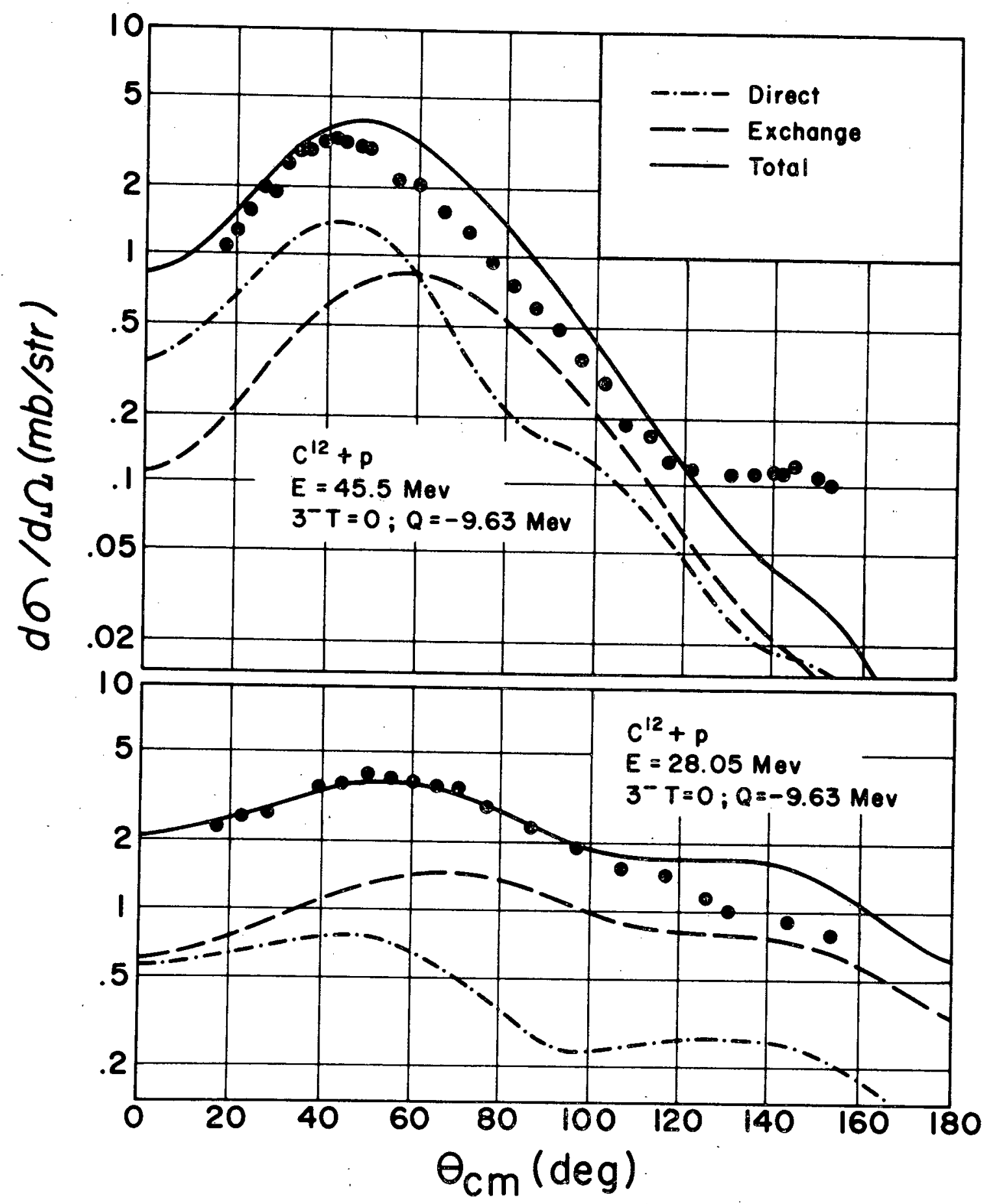

Figure 10.--Same as Figure 7 for $K-K$ force. In addition complete differential cross sections are decomposed into direct and exchange components. 


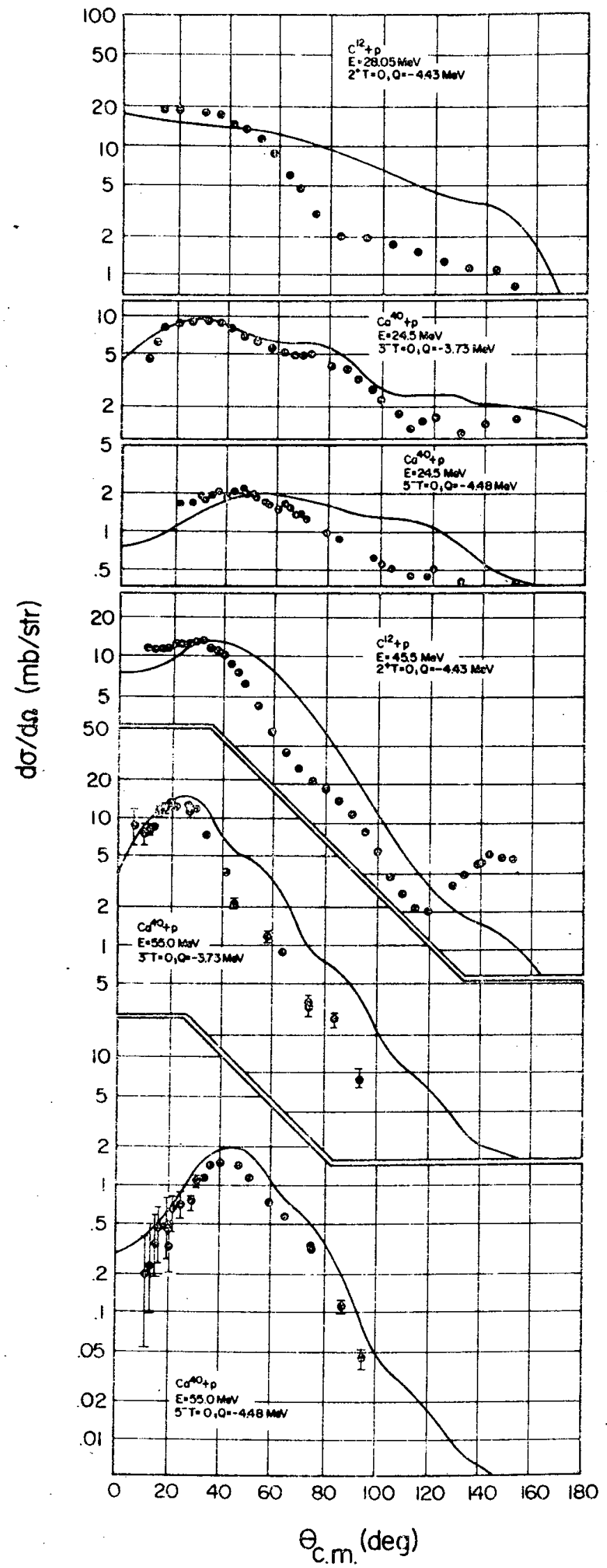

Figure 11.--Same as Figure 8 for $K-K$ force. 


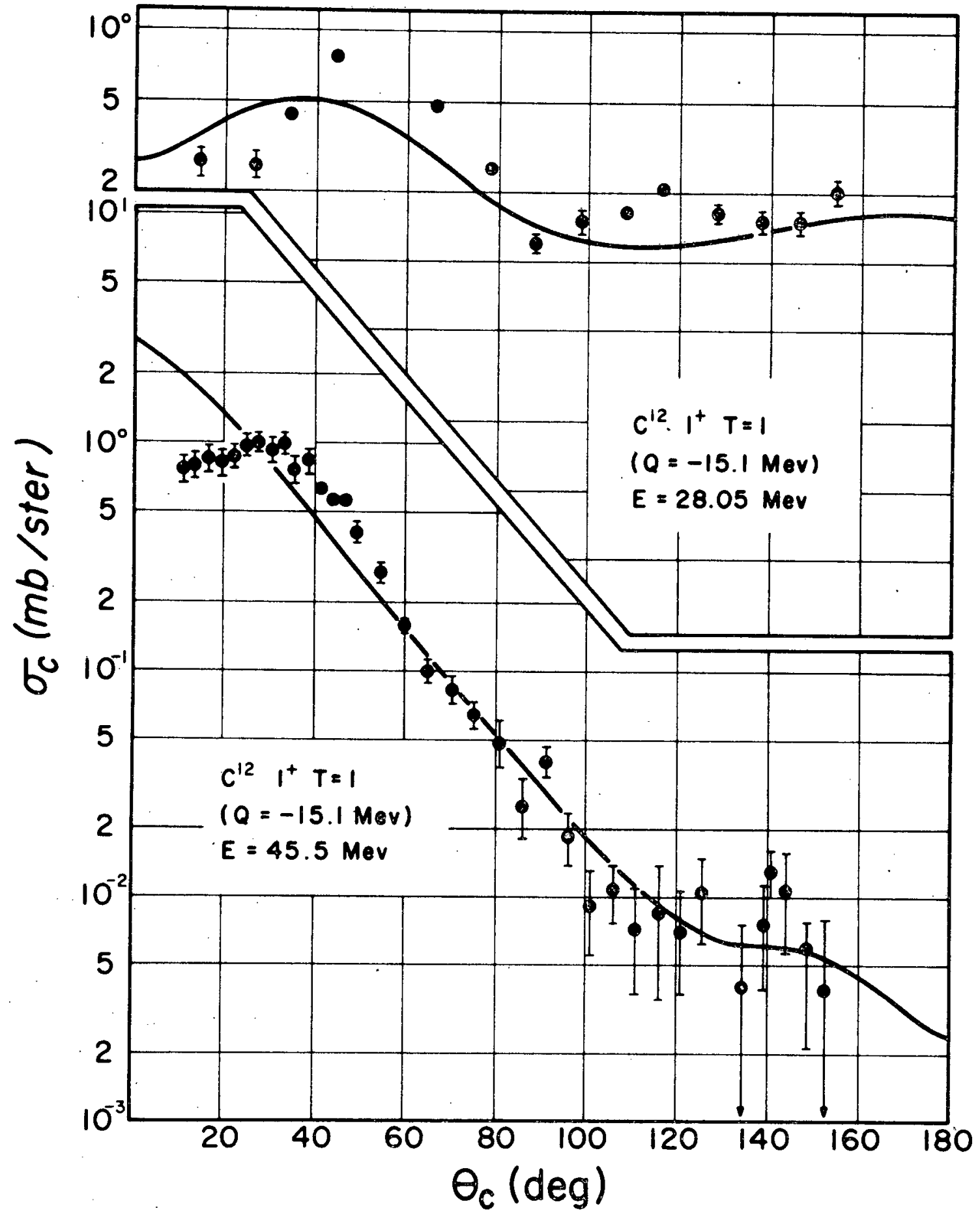

Figure 12.--Same as Figure 6 for Yukawa effective range force. 


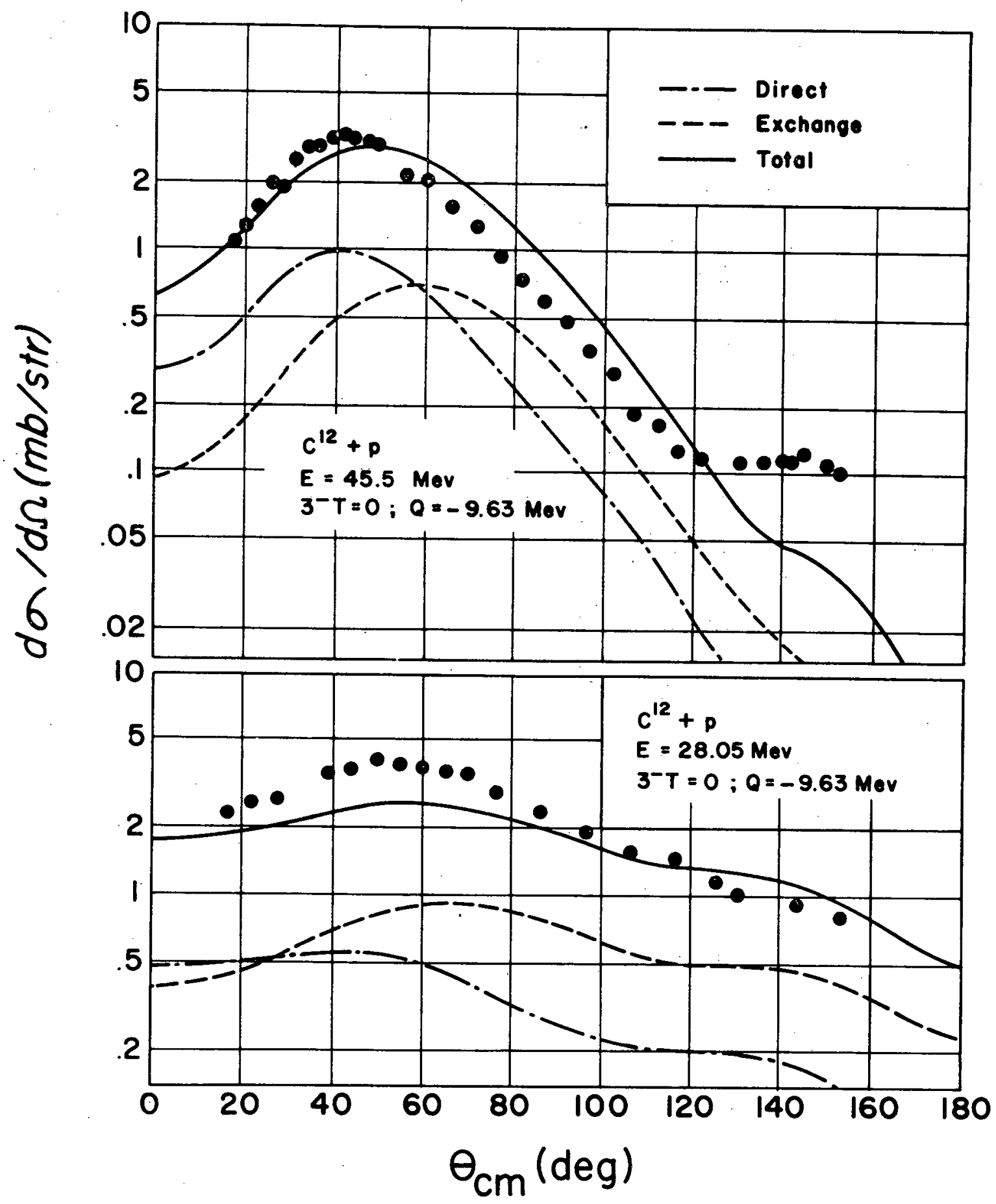

Figure 13.--Same as Figure 10 for Yukawa effective range 


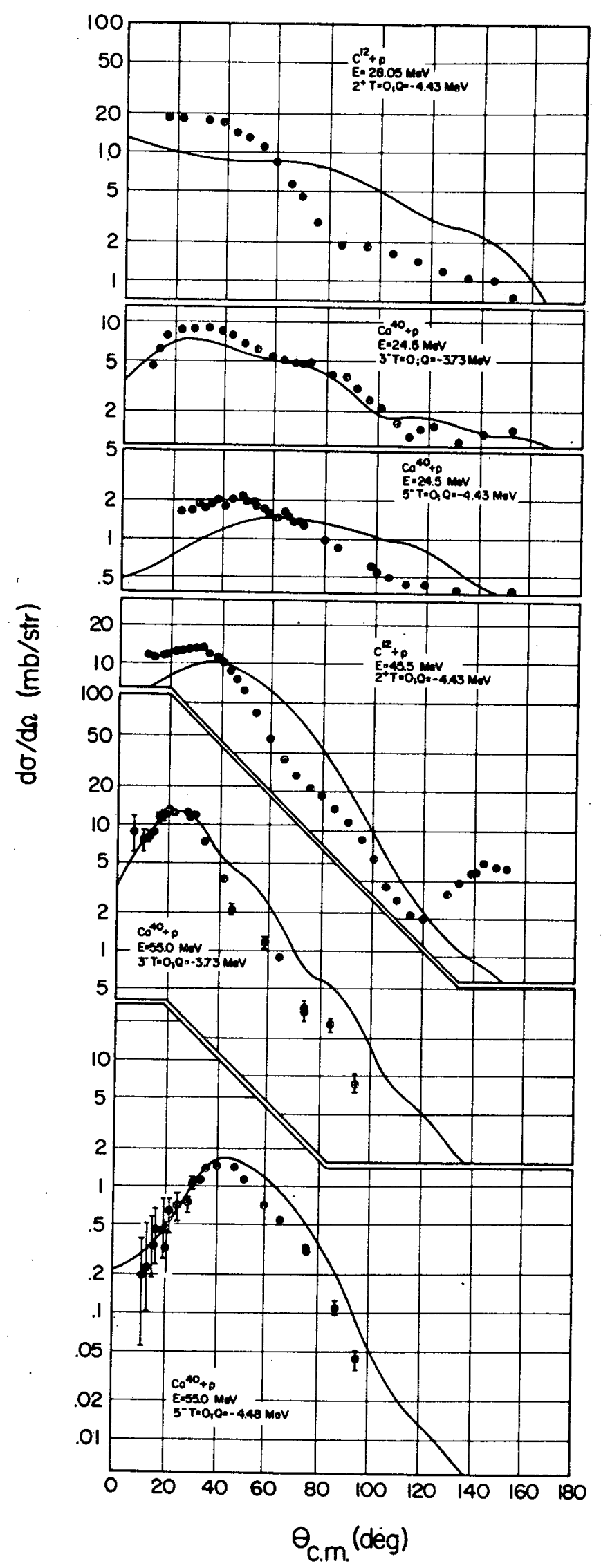

Figure 14.--Same as Figure 8 for Yukawa effective range force. 
TABLE 1.--Optical parameters used in $\mathrm{C}^{12}$ and $\mathrm{Ca}^{40}$ calculations of this work. Well depths are in $\mathrm{MeV}$ and radii and diffuseness parameters are in $F$.

\begin{tabular}{|c|c|c|c|c|c|c|c|c|c|c|c|c|c|}
\hline Target & $E_{L}$ & $\mathrm{~V}$ & W & $w_{0}$ & $r_{0}$ & $a$ & $r_{0}^{\prime}$ & $a^{\prime}$ & $\mathrm{V}_{\mathrm{s}}$ & $r_{\text {so }}$ & $a_{\text {so }}$ & $r_{c}$ & Reference \\
\hline $\mathrm{C}_{12}$ & 28.05 & 48.06 & 0 & 3.92 & 1.13 & .578 & 1.379 & .570 & $9 \cdot 32$ & 1.125 & .573 & 1.20 & 76 \\
\hline$C^{12}$ & 45.5 & 34.5 & 4.9 & 0 & 1.22 & .67 & 1.40 & .70 & $7 \cdot 5$ & 1.22 & 0.67 & 1.20 & 77 \\
\hline $\mathrm{Ca}^{40}$ & 25 & 47.2 & 1.78 & 4.83 & 1.17 & .703 & 1.288 & .653 & 5.59 & 1.17 & .703 & 1.20 & 78 \\
\hline $\mathrm{Ca}^{40}$ & 55 & 41.1 & 7.2 & 0 & 1.18 & .70 & 1.40 & .70 & $7 \cdot 50$ & 1.18 & .70 & 1.20 & $77^{\mathrm{a}}$ \\
\hline
\end{tabular}


TABLE 2.--Integrated cross sections corresponding to results shown in Fig. 6-14. Decomposition of integrated cross section, $\sigma_{T}$, into $\sigma_{d i r}$ and $\sigma_{e x}$ is given for the $K-K$ force and the Yukawa effective range force. All cross sections are in $\mathrm{mb}$.

\begin{tabular}{|c|c|c|c|c|c|c|}
\hline Target & $\mathrm{E}(\mathrm{MeV})$ & $J^{\pi}$ & Force & $\sigma_{d i r}(m b)$ & $\sigma_{\mathrm{ex}}(\mathrm{mb})$ & $\sigma_{T}(\mathrm{mb})$ \\
\hline \multirow{18}{*}{$c^{12}$} & \multirow{9}{*}{28.05} & \multirow{3}{*}{$1^{+}$} & KK & 1.22 & .470 & 3.18 \\
\hline & & & $\mathrm{ER}$ & 1.33 & .303 & 2.86 \\
\hline & & & IA & - & - & 3.05 \\
\hline & & \multirow{3}{*}{$2^{+}$} & $\mathrm{KK}$ & 22.4 & $33 \cdot 5$ & 1.02 \\
\hline & & & $\mathrm{ER}$ & 17.9 & 21.3 & 73.6 \\
\hline & & & IA & - & - & 94.0 \\
\hline & & \multirow{3}{*}{$3^{-}$} & KK & 5.06 & 12.9 & 30.1 \\
\hline & & & $\mathrm{ER}$ & 4.06 & 8.22 & 22.1 \\
\hline & & & IA & - & - & 26.9 \\
\hline & \multirow{9}{*}{45.5} & \multirow{3}{*}{$1^{+}$} & KK & 1.06 & .150 & 1.99 \\
\hline & & & $\mathrm{ER}$ & 1.30 & .122 & 2.18 \\
\hline & & & IA & - & - & 2.08 \\
\hline & & \multirow{3}{*}{$2^{+}$} & $\mathrm{KK}$ & 17.0 & 9.12 & 47.0 \\
\hline & & & $\mathrm{ER}$ & 13.0 & 7.42 & 37.6 \\
\hline & & & IA & - & - & 39.7 \\
\hline & & \multirow{3}{*}{$3^{-}$} & KK & 4.75 & 4.28 & 16.1 \\
\hline & & & $\mathrm{ER}$ & 3.63 & 3.48 & 13.4 \\
\hline & & & $I A$ & - & - & 13.9 \\
\hline & \multirow[b]{3}{*}{25} & \multirow{3}{*}{$3^{-}$} & $\mathrm{KK}$ & 16.1 & 14.6 & 58.2 \\
\hline & & & $\mathrm{ER}$ & 12.2 & 9.14 & 39.6 \\
\hline & & & IA & - & - & 52.9 \\
\hline
\end{tabular}


T'ABLE 2.--Continued.

\begin{tabular}{|c|c|c|c|c|c|c|}
\hline Target & $\mathrm{E}(\mathrm{MeV})$ & $J^{\pi}$ & Force & $\sigma_{\mathrm{dir}}(\mathrm{mb})$ & $\sigma_{e x}(m b)$ & $\sigma_{\mathrm{T}}(\mathrm{mb})$ \\
\hline \multirow{9}{*}{$\mathrm{Ca}^{40}$} & & & KK & 2.21 & 8.35 & 16.9 \\
\hline & & $5^{-}$ & $\mathrm{ER}$ & 1.79 & 5.26 & 12.6 \\
\hline & & & IA & - & - & 14.5 \\
\hline & \multirow{6}{*}{55} & \multirow{3}{*}{$3^{-}$} & KK & 15.5 & 2.51 & 29.4 \\
\hline & & & $\mathrm{ER}$ & 11.7 & 2.44 & 23.7 \\
\hline & & & IA & - & - & 22.6 \\
\hline & & \multirow{3}{*}{$5^{-}$} & $\mathrm{KK}$ & 2.28 & 1.32 & 6.30 \\
\hline & & & ER & 1.69 & 1.28 & 5.64 \\
\hline & & & IA & - & - & 5.15 \\
\hline
\end{tabular}


obtained with the other two forces. Differences bétween the $\mathrm{K}-\mathrm{K}$ force and the Yukawa effective range force were also noted in Ref. 32, i.e. the Yukawa effective range force overestimated the real well depth and the mean square radius of the real part of the optical potential, giving much poorer agreement with phenomenological potentials than the $\mathrm{K}-\mathrm{K}$ force:

The differences between the $K-K$ force and the Yukawa effective range force for these normal parity transitions can be understood from Table 2 and/or comparison of Fig. 10 and 13. From Table 2 it is clear that the values of $\sigma$ dir for these two forces do not show any pronounced energy dependence. The $\mathrm{K}-\mathrm{K}$ force gives slightly larger values of $\sigma_{\text {dir: }}$ The values of $\sigma_{\text {ex }}$ do vary significantly with energy, with those for the $\mathrm{K}-\mathrm{K}$ force exhibiting the sharpest energy dependence. Because of the slower drop-off with energy of $\sigma_{\text {ex }}$ for the Yukawa effective range force, the magnitude of the total differential cross sections it produces catch up with those for the $\mathrm{K}-\mathrm{K}$ force as the energy increases. Differences of this type were suggested in the discussion of these forces in section 6 of chapter 5. It was also pointed out in Section 3 of Chapter 5 that forces of Ionger range than a $1 F$ range Yukawa were necessary to reproduce the energy dependence of the experimental cross sections a condition satisfied by both of the above forces. As a result of the note added to chapter 5 no conclusion will be drawn concerning the significance of the differences between these two forces in relation to the data. This 
note indicates that the approximate treatment of antisymmetrization is better for Yukawa forces than for the $K-K$ force which would leave any conclusion open to question. Recently, Agassi and Schaeffer ${ }^{79}$ have obtained a good fit to the $55 \mathrm{MeV}$ data for the $\mathrm{L}=3$ transition in $\mathrm{Ca}^{40}$. In their calculation antisymmetrization was treated exactly and they used a Serber force of Yukawa form with a range of 1.37F. This force is similar to the Yukawa effective range force used in this work. They used the R.P.A. vector of Ref. 53 to describe this transition. Their result is consistent with this work. They also found that the force CAL, used in the calculation of the state vectors, fails to reproauce the data for this case.

For the abnormal parity transition, Fig. 6, 9, and 12, the magnitude of the theoretical cross sections obtained with all three forces are in reasonable agreement at both energies. Actually, at the lower energy $\sigma_{T}$ for the Yukawa effective range force is slightly smaller than $\sigma_{T}$ for the $K-K$ force. This situation reverses at the higher energy; therefore, the trend is the same as in the other cases. As this is an $\mathrm{L}=0$ transition exchange is not as important. Further the values of $\sigma_{\text {dir }}$ for the Yukawa effective range force are larger than those for the $K-K$ force which is a reversal of the results for the normal parity transitions. This is simply a reflection of the differencesbetween the forces at large radii. For the $\mathrm{K}-\mathrm{K}$ force $\sigma_{\text {dir }}$ decreases a little with energy and $\sigma_{\text {dir }}$ for the Yukawa effective range force remains 
almost constant. Unlike the normal parity transitions, there are noticeable shape differences in the theoretical differential cross sections for this transition with the experimental data favoring the results obtained with the impulse approximation pseudo-potential. It is concluded that the cross sections for this transition are sensitive to the precise shape and phase of the two-body force.

The theoretical cross sections have a tendency to fall off too slowly with increasing angle and they don't show enough structure. No attempt has been made to try and improve the shape agreement between the theoretical and experimental angular distributions. It is known that better shapes would result if the theoretical form factors could be pushed out radially. The density dependence and the imaginary part of the projectile-target interaction might produce this effect.

It has been observed in many cases that the direct cross sections computed with the $K-K$ force show good shape agreement with the experimental angular distribution. This shape agreement is then lost when the exchange component is included. This does not happen with the Yukawa effective range force.

The reason for this is that the direct form factors for the $\mathrm{K}-\mathrm{K}$ force are more surfaced peaked than those for the Yukawa effective range fcrce. This is evident in Fig. 15 where the direct form factors for the $L=2$ transitions in $c^{12}$ are compared. Also shown are the complete form factors (with exchange) for $28.05 \mathrm{MeV}$. The total form factors peak 


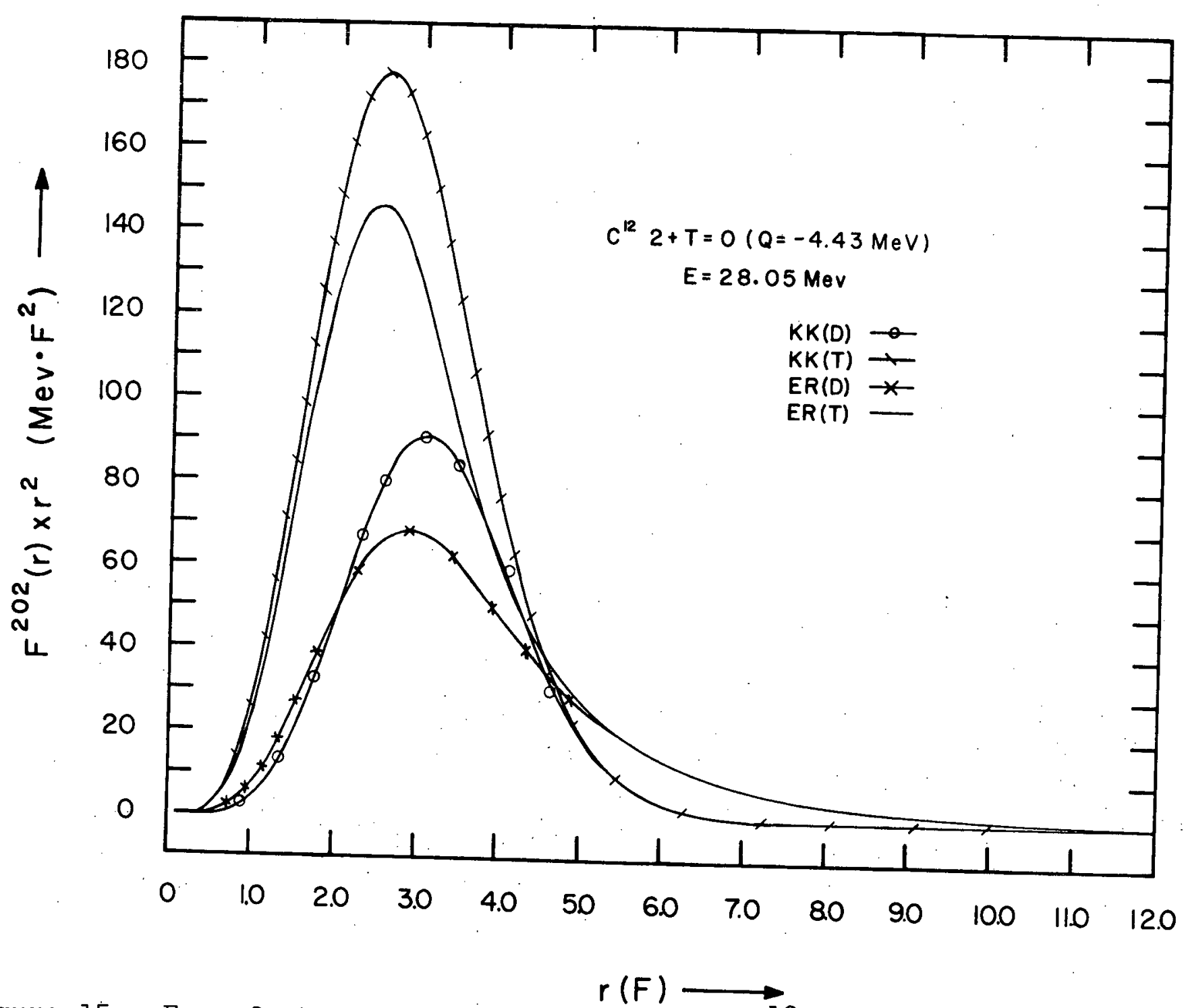

Figure 15.--Form factors for $\mathrm{L}=2$ transition in $\mathrm{Cl}^{12}$ as obtained with the $\mathrm{K}-\mathrm{K}$ force and the Yukawa effective range force. Direct form factors (D) are shown as well as total form factors (T) for $28.05 \mathrm{MeV}$. 
well inside the direct form factors. The difference in peak positions for the direct form factors in this figure is about $.4 \mathrm{~F}$, whereas this difference for the total form factors is only about.lF. The latter accounts for the similarity of the final results for the two forces. The long tail on the form factors for the Yukawa effective range force does not aid in giving better shapes.

The cross sections shown in Fig. 10 and 13 are not extremely good examples of the above point: Here the total cross sections show fairly good shape agreement with the data out to at least 100 degrees. The direct cross sections show too much structure. It is noted, however, that the $\mathrm{K}-\mathrm{K}$ direct cross sections show more structure than those for the Yukawa effective range force which is consistent with form factor differences like those djsplayed in Fig. 15. It would appear that some of the deficiencies in the angular distributions of Fig. 6-14 are attributable to deficiencies in the transition densities. In particular, the fact that the angular distributions for the $L=3$ transition in $\mathrm{C}^{12}$ and those for the $\mathrm{L}=5$ transition in $\mathrm{Ca}^{40}$ peak at too large an angle appears to be consistent with the $\left(e, e^{\prime}\right)$ results which have been shown. The impulse approximation pseudo-potential and the Yukawa effective range force yield cross sections for the $\mathrm{L}=2$ transition in $\mathrm{C}^{12}$ which fall under the data. The $\left(e, e^{\prime}\right)$ results suggested this. The $K-K$ cross sections do not reproduce this discrepancy. 
As a result of the uncertainties in the approximate treatment of antisymmetrization it is suspected that the magnitude of the differential cross sections for the $K-K$ force might be overestimated appreciably, at least at the lower energies. This effect will be greatest for the $L=0$ transition and will become less important with increasing $\mathrm{L}$. It has already been suggested that the $L=2$ result is being overestimated from the comparison of the $\left(e, e^{\prime}\right)$ and $\left(p, p^{\prime}\right)$ calculations. It has recently been indicated that the tensor force might be important for the abnormal parity $L=0$ transition. 80 Including it is found to improve the shape agreement between theory and experiment at $45.5 \mathrm{MeV}$, particularly at forward angles. It may be that the approximate treatment of exchange is masking the need for this contribution to this transition.

\section{Section B}

\section{Target $\mathrm{Li}^{6}$}

The $\mathrm{J}^{\pi}$, T values for the first three states of $\mathrm{Li}^{6}$ are $1^{+}, 0 ; 3^{+}, 0$; and $0^{+}, 1.14$ The second state is observed at 2.18 MeV above the first which is the ground state. The third lies $3.56 \mathrm{MeV}$ above the ground state. Differential cross sections have been measured for the $\mathrm{Li}^{6}\left(\mathrm{p}, \mathrm{p}^{\prime}\right) \mathrm{Li}^{6 *}$ $(Q=-2.18$ and $-3.56 \mathrm{MeV})$ reactions at $24.4 \mathrm{MeV} .81$ Theoretical cross sections have been calculated using the $K-K$ force. Shell model, LS-coupled wave functions have been used to describe the target and the value $\alpha=.581 \mathrm{~F}^{-1}$ has been assumed 
for the harmonic oscillator constant. 14 The optical parameters are also given in Ref. 14. For the $Q=-2.18 \mathrm{MeV}$ transition only the contribution from the triad $(202,0)$ is important and only the triad $(011, I)$ is allowed for the $Q=-3.56 \mathrm{MeV}$ transition; therefore, the components of the force which are involved are $t_{00}$ and $t_{11}$, respectively. The results are shown in Fig. 16.

The agreement between theory and experiment is poor. The $L=2$ cross section is badly underestimated and the $L=0$ cross section is overestimated. In addition, the latter result does not show any of the structure displayed in the data. Similar agreement with experiment is obtained when these wave functions are used in the analysis of the $\left(e, e^{\prime}\right)$ reaction. 82 Fig. 17 shows a rough fit ${ }^{83}$ to the experimental (e,e') form factor 84 for the $L=2$ transition. Adjacent to it is the result which is obtained using the transition density, empirically determined from this fit, to calculate the corresponding ( $\left.p, p^{\prime}\right)$ cross section with the assumption that the transition still goes through the $t_{00}$ part of the $K-K$ force. The correspondence between the $\left(e, e^{\prime}\right)$ and $\left(p, p^{\prime}\right)$ results is good and it is concluded that the LScoupled wave functions do not give a good description of the target.

Excellent fits to inelastic electron scattering data have been obtained, for both the transitions under discussion, on the basis of the cluster model. ${ }^{82}$ A parallel analysis of the $\left(p, p^{\prime}\right)$ data is planned. This possibly could 

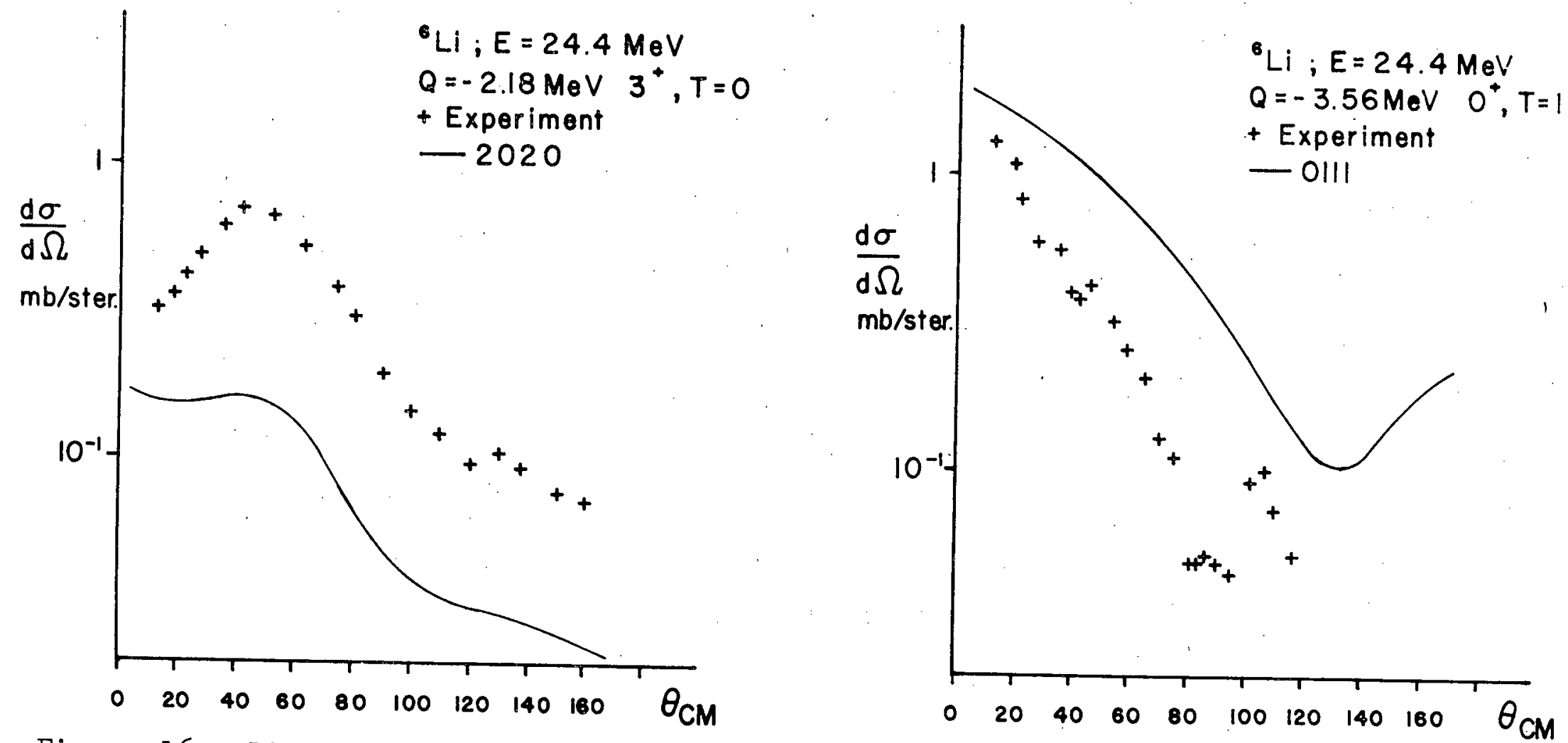

Figure 16.--Differential cross sections for the excitation of the first two excited states of $\mathrm{Li}^{6}$ by $24.4 \mathrm{MeV}$ protons. The $\mathrm{K}-\mathrm{K}$ force has been used in the theoretical calculations and shell model, LS-coupled wave functions have used to describe the target. 


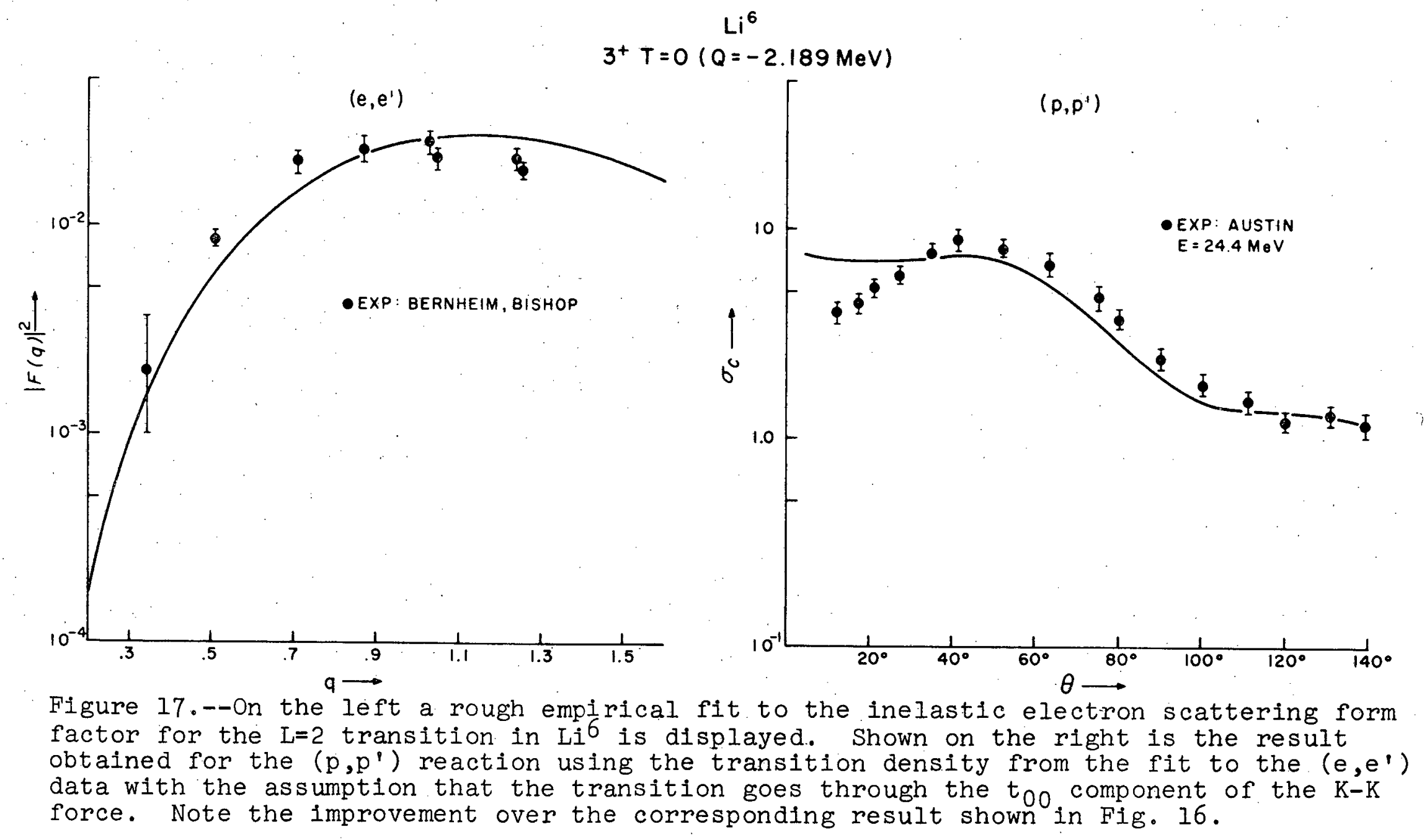


be extended to transitions which have been observed in neighboring nuclei. It may be necessary to improve the treatment of antisymmetrization and to include the tensor force in this work, particularly for the case of the $L=0$ transition. These points were previously made with respect to the $L=0$ transition in $\mathrm{C}^{12}$ which was discussed in section $A$ of this chapter.

Target $\mathrm{C}^{12}$

There is a $2^{+} \mathrm{T}=1$ state in $\mathrm{C}^{12}$ at $\mathrm{Q}=-16.1 \mathrm{MeV}$. The triads $(202,1)$ and $(212,1)$ can contribute to the excitation of this level in the ( $\left.p, p^{\prime}\right)$ reaction. The components of the projectile-target interaction which are involved are $t_{01}$ and $t_{11}$, respectively. Both triads make appreciable contributions to the cross section as is seen in Fig. 18. This is to be contrasted with the situation for normal parity $\mathrm{T}=0$ transition where only the non-"spin-flip" contributions were found to be important. Here the impulse approximation pseudo-potential has been used with the R.P.A. vector of Ref. 50. The data is from Ref. 72 and all parameters are fixed as in the previous $\mathrm{C}^{12}$ calculations. The total cross section shown has been obtained by summing the $(202,1)$ and $(212,1)$ components incoherently. No significant change occurs when a coherent sum is performed. The magnitude of the theoretical result is in reasonable agreement with experiment, but the shape is quite poor. A comparable fit 


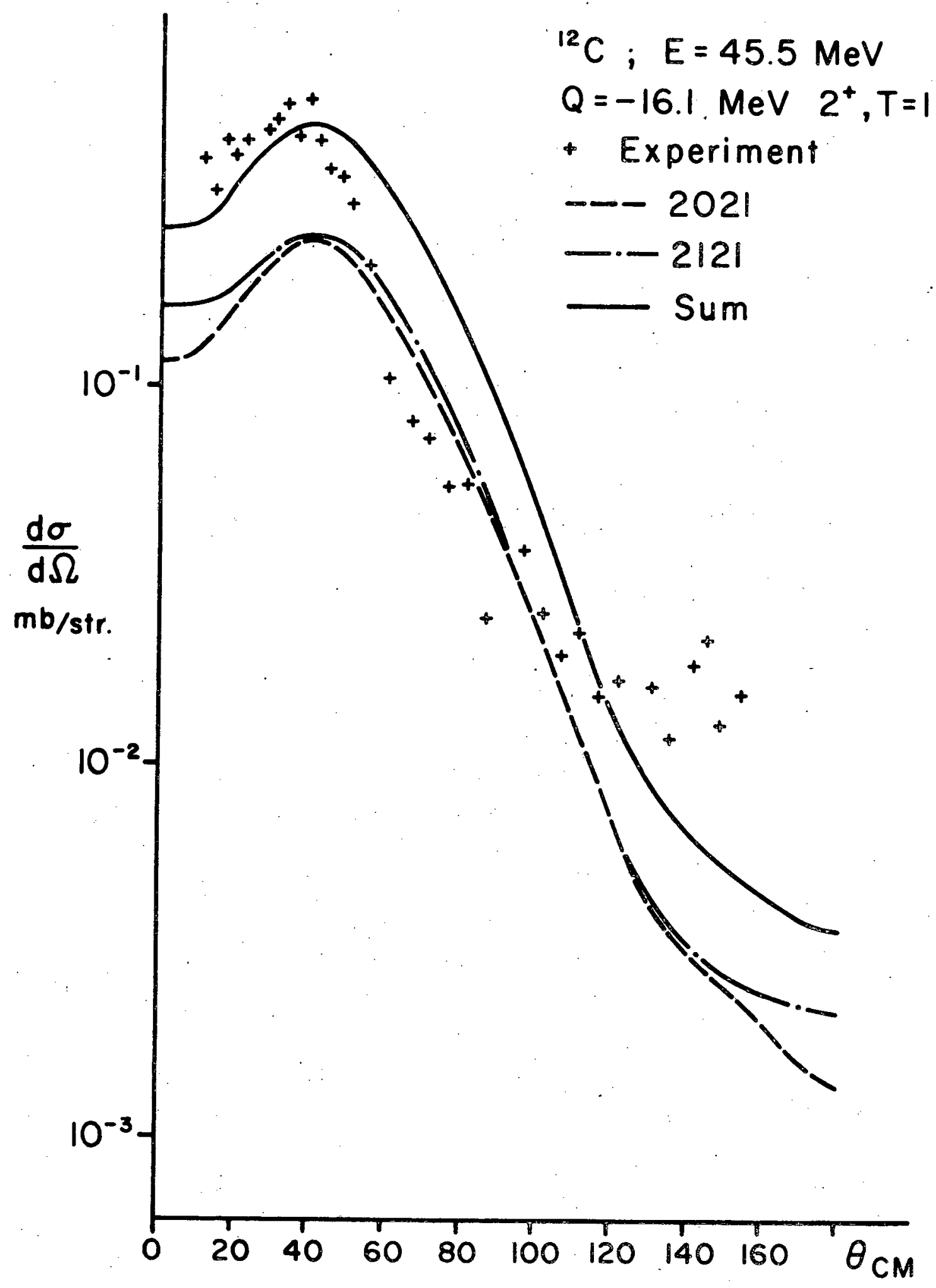

Figure 18.--Comparison of theoretical and experimental differential cross sections for the excitation of the $2^{+} T=I(Q=-16.1$ $\mathrm{MeV})$ level of $\mathrm{C}^{12}$ by $45.5 \mathrm{MeV}$ protons. The impulse approximation pseudo-potential is used for the projectile-target interaction. 
to the $156 \mathrm{MeV}\left(\mathrm{p}, \mathrm{p}^{\prime}\right)$ data has been obtained using this vector, 42,43 so this result is an indication that the $t_{0 I}$, component of the "realistic" interactions is not unreasonable.

'l'arget $0^{16}$

Fig. 19 shows the theoretical result obtained with the $K-K$ force for the excitation of the $3^{-} \mathrm{T}=0(\mathrm{Q}=-6.13 \mathrm{MeV})$ level of $\mathrm{O}^{16}$ by $24.7 \mathrm{MeV}$ incident protons. The data is the same as that shown in Fig. 5.10. This is an $L=3$ transition which goes through the $t_{00}$ component of the force. The R.P.A. vector of Ref. 50 was used in the calculation and the harmonic oscillator constant was set at $\alpha=.559 \mathrm{~F}^{-1}$. The agreement between theory and experiment is good; however, since this calculation was performed better optical parameters have been obtained and it has been shown that the Gillet vector does not give a good fit to the inelastic electron scattering form factor. 73 correcting these deficiencies leads to a theoretical result which falls about a factor of 1.5 below the data. An explanation of this discrepancy is not presently available.

Target $\mathrm{Ca}^{40}$

Theoretical cross sections have been calculated for the excitation of the $3^{-} \mathrm{T}=0(\mathrm{Q}=-6.28 \mathrm{MeV})$ and the $2^{-} \mathrm{T}=0(\mathrm{Q}=-6.02$ MeV) states in $\mathrm{Ca}^{40}+\mathrm{p}$ at $24.5 \mathrm{MeV}$. These are preliminary results which have been obtained in a study of the $\mathrm{Ca}^{40}\left(\mathrm{p}, \mathrm{p}^{\prime}\right)$ $\mathrm{ca}^{40 *}$ data collected by $\mathrm{C}$. Gruhn and collaborators. The 


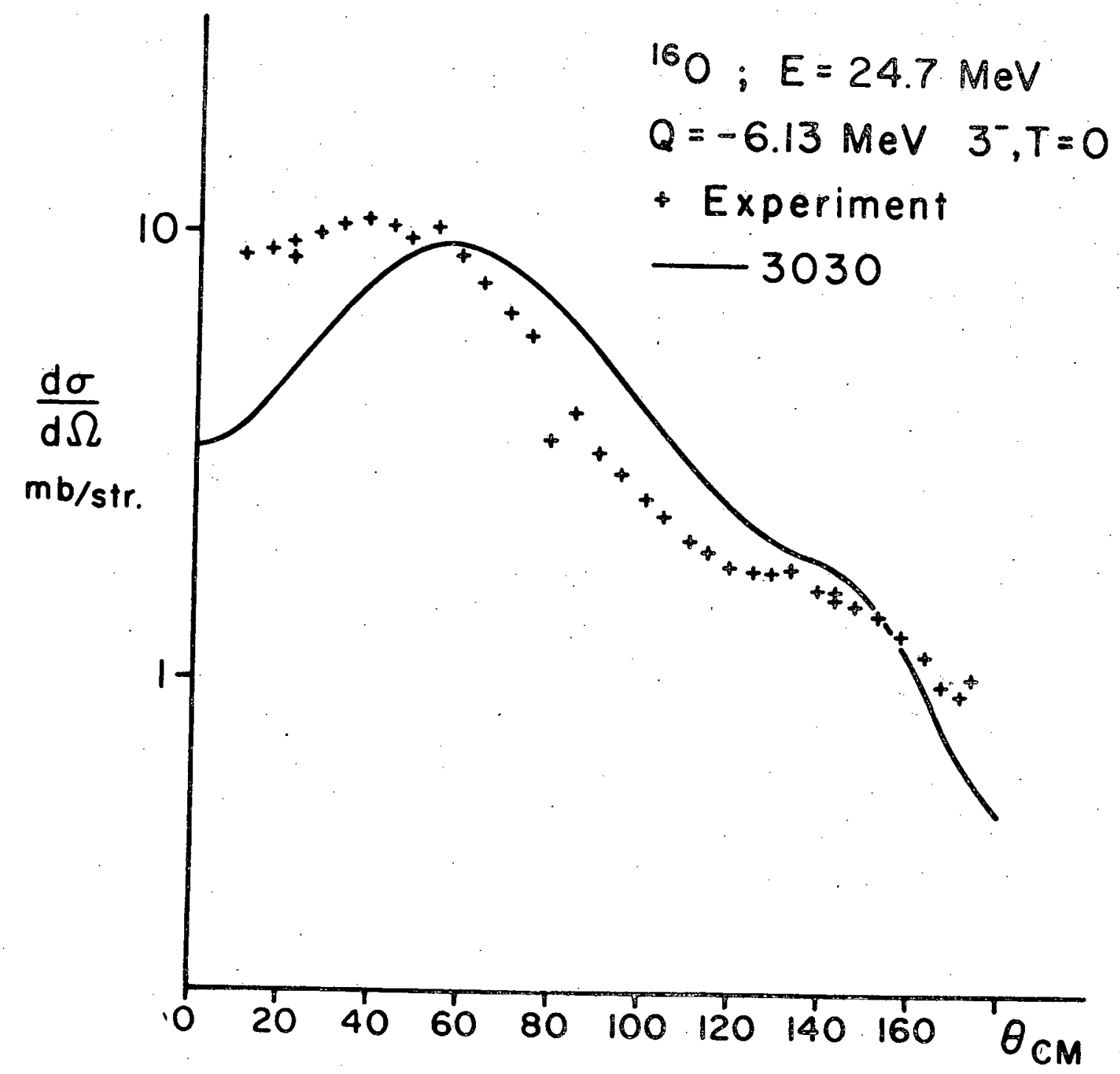

Figure 19.--Comparison of theoretical and experimental differential cross sections for the excitation of $3^{-} \mathrm{T}=0(\mathrm{Q}=-6.13 \mathrm{MeV})$ level of 016 by $24.7 \mathrm{MeV}$ protons. The $\mathrm{K}-\mathrm{K}$ force is used for the projectile-target interaction. 
impulse approximation pseudo-potential has been used in these calculations, R.P.A. vectors are from Ref. 54, and all parameters are fixed as before. Only the triads $(303,0)$ and $(112,0)$ have been considered and these transitions go through $t_{00}$ and $t_{10}$, respectively.

The $L=3$ cross section is shown on the left in Fig. 20 where it is compared with the result shown previously for the excitation of the first $3^{-} \mathrm{T}=0$ state in $\mathrm{Ca}^{40}$. There is a noticeable difference in the shape of the two experimental angular distributions. This difference is not related to the difference in $Q$ for the two transitions. The magnitude of the cross section for the second $L=3$ excitation is an order of magnitude lower tian that for the first. The theoretical calculations reproduce the data quite well. In detail the change in shape comes about because of differences in the dominant configurations of the R.P.A. vectors, $1 . e$. the If $7 / 2^{-l d_{3 / 2}}$ particle-hole pair is the largest component of the first state vector (see Fig. 4) while it is the $2 \mathrm{p}_{3 / 2}$ $1 d_{3 / 2}$ particle-hole pair which is most important in the second. Because of the node in the $2 \mathrm{p}_{3 / 2}$ radial wave function, the transition density for the second excitation is large and negative in the interior and has a positive peak just outside the nuclear surface. From Fig. 4 it is seen that the transition density for the first excitation is small and negative in the interior and has a dominant positive peak just inside the surface. The former simulates a somewhat larger diffracting object and hence the cross section for 


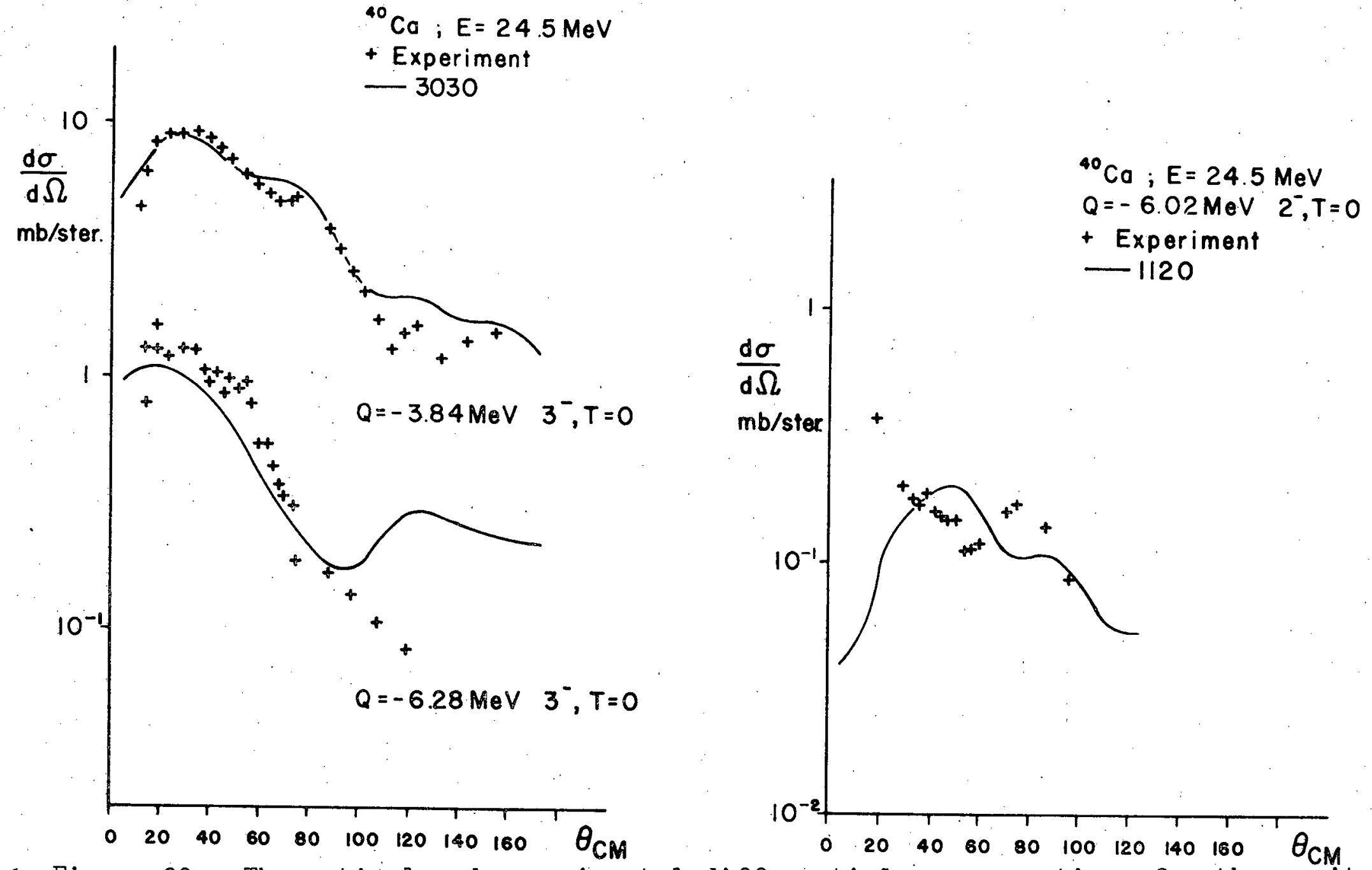

- Figure 20.-Theoretical and experimental differential cross sections for the excitation of the $3^{-T}=0 \quad(Q=-6.28 \mathrm{MeV})$ and $2-T=0 \quad(Q=-6.02 \mathrm{MeV})$ levels of $\mathrm{Ca} 40$ by $2.4 .5 \mathrm{MeV}$ protons. Results for excitation of first $3^{-\mathrm{T}}=0$ level in $\mathrm{Ca} 40$ are also shown for comparison. The impulse approximation pseudo-potential is used for projectile-target interaction. 
this case falls off faster with increasing angle. This is an amusing comparison as it demonstrates some sensitivity to a particular detail of the target wave function.

The result for the $L=1$ transition is shown on the right in Fig. 20. The magnitude of the theoretical cross section is seen to be in reasonable agreement with experiment, but there is no apparent correlation in shape. The R.P.A. says that this state is almost a. single $I f_{7 / 2}-1 \alpha_{3 / 2}$ particle-hole pair. It would be interesting to examine the effect of the tensor force in this transition.

Target $\mathrm{Pb}^{208}$

Theoretical differential cross sections have been calculated for the excitation of the $3^{-}(Q=-2.62 \mathrm{MeV})$ and $5^{-}(\mathrm{Q}=-3.11 \mathrm{MeV})$ levels of $\mathrm{Pb}^{208}$ at $40.0 \mathrm{MeV}$ and $24.5 \mathrm{MeV}$, respectively. Experimental data for the former transition is given in Ref. 77 and 85 and in Ref. 11 for the latter. transition. Optical parameters used in the calculations are to be found in these same references. The $K-K$ force is used, the R.P.A. vectors are from Ref. 52, and $\alpha$ was taken to be $.405 \mathrm{~F}^{-1}$. The results are compared with the data in Fig. 21. The agreement between theory and experiment for the $L=3$ transition is not bad, but the $L=5$ result falis a factor of $2-3$ below the data.

The proton and neutron $L=3$ transition densities are almost identical; therefore, this transition tests the $t_{00}$ component of the force. Using this same vector to calculate 


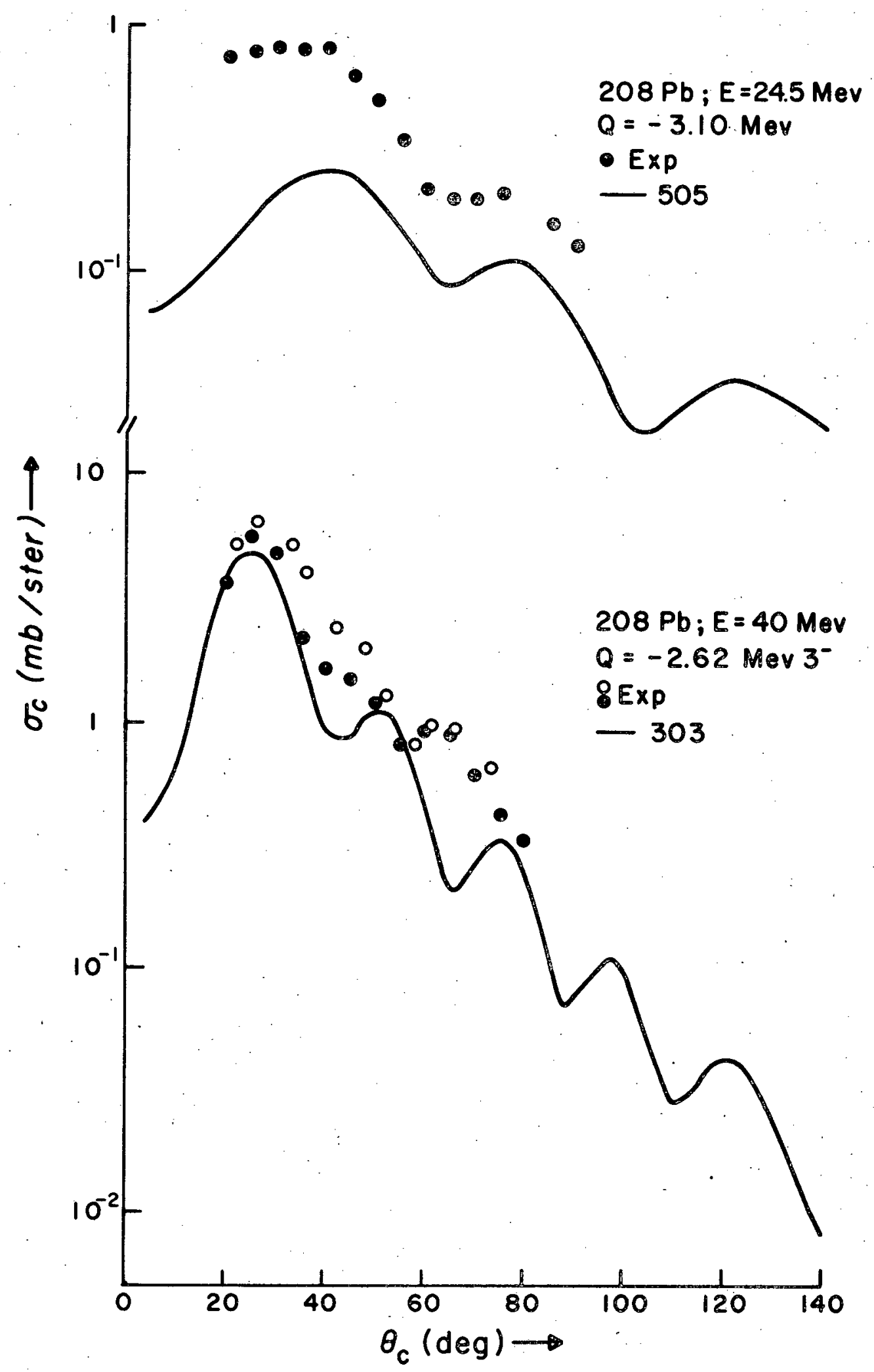

Figure 2l.--Theoretical and experimental differential cross sections for $3^{-}(Q=-2.62 \mathrm{MeV})$ and $5^{-}(Q=-3.10 \mathrm{MeV})$ levels in $\mathrm{Pb}^{208}$ by 40 and $24.5 \mathrm{MeV}$ protons, respectively. For the $\mathrm{L}=3$ transition the dots are the data points from Ref. 77 and the circles are the data points from Ref. 85 . The $\mathrm{K}-\mathrm{K}$ force is used for the projectile-target interaction. 
the form factor for inelastic electron scattering, a fit to the data is obtained which is comparable to that shown in Fig. 21.83 Nothing can be said about the $L=5$ transition as there is no $\left(e, e^{\prime}\right)$ data available although the poor result is probably a reflection of a deficiency in the R.P.A. vector for this transition.

\section{$1^{-} \mathrm{T}=0$ Excitations}

$\mathrm{C}^{12}$ is known to have a $\mathrm{I}^{-} \mathrm{T}=0$ level at $\mathrm{Q}=-10.8 \mathrm{MeV}$ and the same $J^{\pi}, T$ is assigned to the $Q=-5.90 \mathrm{MeV}$ level in $C^{40}$. R.P.A. vectors are avallable for these states in Ref. 50 and 54, respectively. These vectors contain a spurious component which represent translational motion of the center of mass of the target rather than internal excitation of the target. These vectors have been "cleaned" by constructing the corresponding spurious states 86,87 and projecting them out. Theoretical results obtained with the $\mathrm{K}-\mathrm{K}$ force, using both the original vectors (spurious) and the clean vectors, are compared with each other and with the data in Fig. 22. The magnitude of the cross sections is not given satisfactorily in these calculations, but it is interesting that the clean vectors reproduce the shape of the experimental angular distributions quite well as compared to the spurious vectors. As the projection technique is not rigorous it is difficult to say more about these results. 

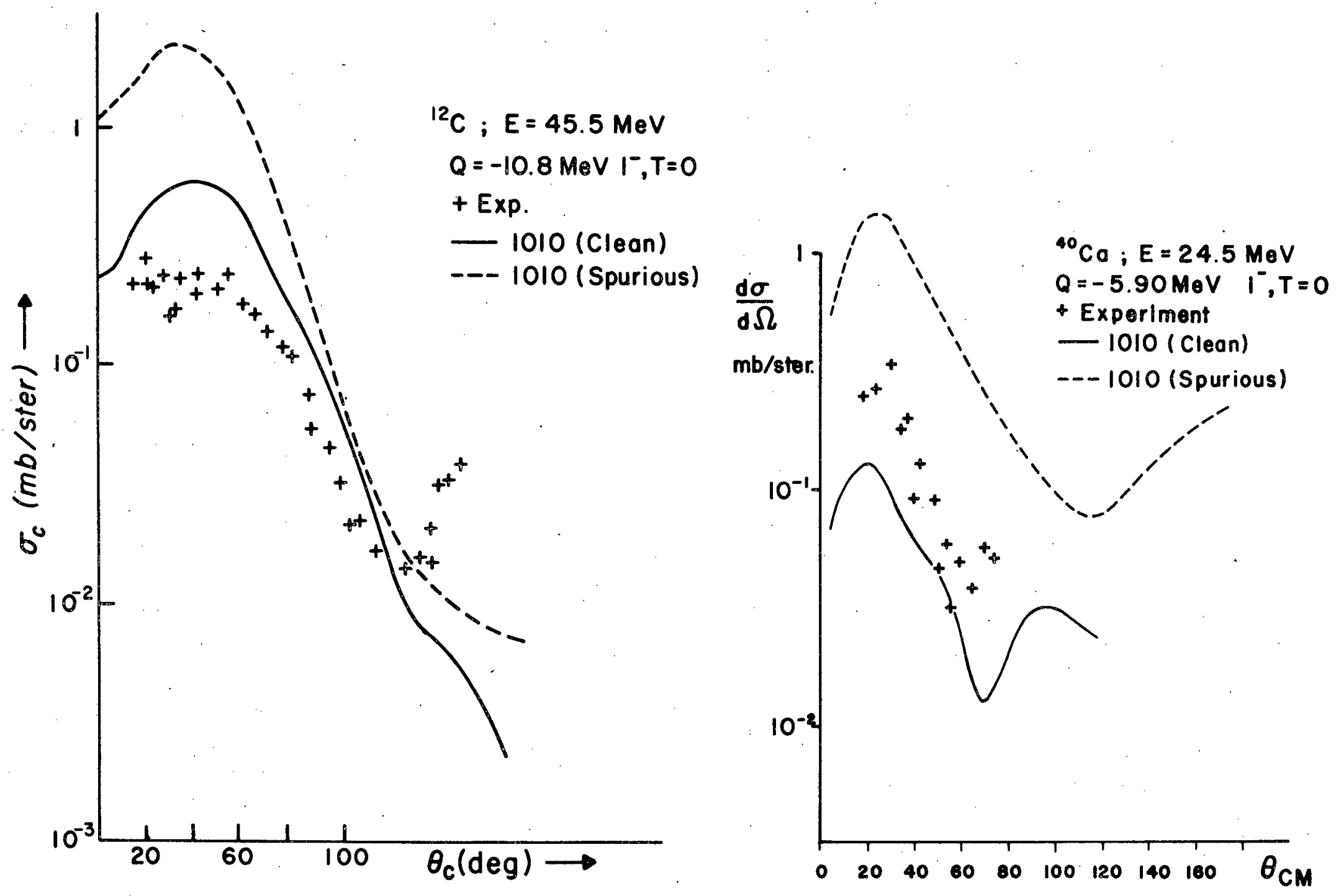

Figure 22.-Comparison between theory and experiment for excitation of $1-T=0$ levels in $\mathrm{Cl}^{12}$ and $\mathrm{Ca} 40$. The effect of projecting out the spurious state is illustrated. 
CHAPTER 7

\section{CORE POLARIZATION IN INELASTIC \\ PROTON-NUCLEUS SCATTERING}

\section{Introduction}

In this chapter the calculations are extended to $\left(p, p^{-}\right)$ transitions involving low lying states in nuclei which possess one or two nucleons outside of a closed shell. Ihe importance of core polarization on the low lying spectra of these nuclei and in these transitions has been discussed by many authors. Several methods have been used for estimating these effects which can be expressed most simply as a renormalization of operators acting on the valence nucleons, e.g. the effective two-body force between valence nucleons and the effective charge of a valence nucleon.

One method is a perturbative treatment of the particlehole excitations of the core which are induced by the valence nucleons. This is carried out to lowest order and particlehole excitations up to about $2 \hbar \omega$ in energy are included.

In following this procedure the interaction of one core nucleon with another core nucleon is neglected, i.e. a zeroth-order shell model Hamiltonian describes the core. 
As the interaction between core nucleons is responsible for the existence of low lying collective states in the core nucleus, it is clear that this method does not include the contributions from these states.

This approach is used by Kuo and Brown $20-25,54$ in their attempt to explain the spectra of nuclei with one or two valence nucleons. They have shown, looking in a systematic way at nuclei in the vicinity of $0^{16}, \mathrm{Ca}^{40}, \mathrm{Ca}^{48}, \mathrm{Ni}^{56}$, $\mathrm{Sr}^{88}$, and $\mathrm{Pb}^{208}$, that core polarization gives rise to a strong pairing effect which is the major feature of the observed spectra. Horie and Arima were among the first to use this method in their calculation of quadrupole moments. 57 Recently, Federman and Zamick have used this model to examine some of the properties of the effective charges for quadrupole transitions for nucleons outside of $\mathrm{Ca}^{40}$ and $\mathrm{Ni}^{56}$ cores. ${ }^{88}$ These studies have been extended to other nuclei ${ }^{89}$ and additional efforts have been directed at estimating the validity of neglecting low-lying collective states of the core nucleus. 90,91

An alternative method is to use the macroscopic vibrational model to describe the core. $92,93,15,16$ The interaction between the valence nucleons and the core is treated in a manner completely analogous to that discussed in Section 2 of Appendix $B$ where the interaction of a projectile with a nucleus, so described, was considered. The eigenstates of the macroscopic vibrational Hamiltonian need not correspond to physical states of the core as the model is used as a vehicle for parameterization. Under the 
assumption that the core strength is at an energy large compared to any of the energy differences between the valence nucleons involved, the role of a given core multipole in the core polarization process is flxed by a single parameter, $C_{L}$, the stiffness parameter for multipole $L$. The renormalization of the two-body forces between nucleons outside the core (bound and/or unbound) as well as the effective charge are easily expressed in terms of these parameters. Using this method, and fixing the $\mathrm{C}_{\mathrm{L}}$ on the basis of empirical effective charges, Love and Satchler 15,16 have demonstrated that core polarization can give a very important, even dominant, contribution to $\left(p, p^{-}\right)$cross sections.

Another variant is to consider the coupling of the valence nucleons to low lying physical states of the core. The macroscopic vibrational model can be used to parameterlze the physical core states, although more consistent calculations would use microscopic wave functions for the core states--such as R.P.A. vectors: The energies of these core states are often comparable with the energy differences between valence nucleons and this has to be taken into account. This method has been used extensively in the lead region.93,94 Calculations of this type are useful in examining the particle-hole model with respect to neglecting low-lying collective states of the core. 
Atkinson and Madsen have given yet another procedure for relating the effect of core polarization in electromagnetic transitions to the effect in the $\left(p, p^{\prime}\right)$ reaction. 19 All these models are attempts to enlarge the vector space used in shell model calculations in an easy to handle way and, at the moment, rest on a very empirical rather than theoretical foundation. These models are discussed in more detail in Appendix D. At any rate the main purpose of the present chapter of this paper is to extend, to the scattering problem, the microscopic perturbative calculation of Kuo and Brown.

Due to the selection rules, transitions generally give more detailed information about the nature of core polarization than bound state calculations. For example, consider a nucleus with two like valence nucleons which are restricted to the $(j)^{2}$ configuration. Such a nucleus will have a $0^{+}$ground state. It is shown in Appendix $D$ that the pairing effect on the ground state binding energy is due to the coherent effect of a number of core multipole excitations, whereas transitions between the states of the $(j)^{2}$ configuration which start or end at the ground state depend essentially on only one core multipole. The $\left(p, p^{\prime}\right)$ reaction is particularly useful for studying core polarization since the available experimental data, unlike that for electromagnetic transition rates, is not limited primarily to quadrupole and octupole transitions. 
$\mathrm{Ti}^{50}, \mathrm{Zr}^{90}, \mathrm{Y}^{89}$, and $\mathrm{Bi}^{209}$ are the nucle1 considered in this paper. The first two have two valence protons and the last two have a single valence proton. In all cases the $3 p-I h$ (or $2 p-I h)$ components of the target wave functions are included as prescribed in Appendix D. The $K-K$ force is used as the interaction between core and valence nucleons. Angular distributions for the $\left(p, p^{\circ}\right)$ reaction and effective charges are calculated and compared with experiment. In the $\left(p, p^{\prime}\right)$ calculations the $K-K$ force is also used for the projectile target interaction. These calculations constitute an attempt to reproduce the (p,p') experimental data from a completely microscopic model with the assumption that the projectile and target nucleons all interact via the same force which in turn is closely related to the free two-nucleon potential.

As an example of a particularly convenient way to relate the effect of core polarization on the spectrum and in transitions, calculations are carried out for $\mathrm{Ti}^{50}$ and $\mathrm{Zr}^{90}$ using the macroscopic vibrational description of the core and fixing the core parameters from the bound state matrix elements of Kuo and Brown. This procedure is discussed in Appendix D. All results are reviewed with respect to coupling to physical core states and in light of the empirical formula of Madsen and Atkinson. A very interesting result is obtained in the case of $\mathrm{BI}^{209}$ where it is found that the transition considered is dominated by a single core phonon. 
2. Calculations and Results $-\mathrm{Ti}^{50}, \mathrm{Zr}^{90}, \mathrm{Y}^{89}$

Macroscopic Vibrational Model and Relation between Core Polarization in Spectra and Transitions $\left(\mathrm{Zr}^{90}\right.$ and $\left.\mathrm{Ti}^{50}\right)$

In $\mathrm{Zr}^{90}$ the transitions from the $0^{+}$ground state to the $0^{+}, 2^{+}, 4^{+}, 6^{+}$, and $8^{+}$states with $Q=-1.75,-2.18$, $-3.07,-3.45$, and $-3.58 \mathrm{MeV}$, respectively, for $18.8 \mathrm{MeV}$ incident protons are considered. The transitions from the $0^{+}$ground state to the $2^{+}$and $4^{+}$levels of $\mathrm{Ti}^{50}$ with $\mathrm{Q}=-1.55$ and $-2.68 \mathrm{MeV}$ for 17.5 and $40 \mathrm{MeV}$ incident protons are also treated. The two $0^{+}$levels in $2 r^{90}$ result from the mixing of the $\left(1 g_{g / 2}\right)^{2}$ and $\left(2 p_{1 / 2}\right)^{2}$ proton configurations where the ratio of $g$ to $p$ amplitudes in the ground state is about three quarters. This ratio has been fixed both theoretically and experimentally. 8,9,54,95,96 The $2^{+}, 4^{+}, 6^{+}$, and $8^{+}$states in question in this nucleus are due to two protons in the $\lg _{9 / 2}$ orbit. The states in $\mathrm{Ti} 50$ are described as two valence protons in the $1 \mathrm{f}_{7 / 2}$ shell. There is also a $6^{+}$state due to this configuration, but it has not been resolved in inelastic proton scattering experiments.

For these cases the multipole decomposition of the $3 p-1 h$ contributions to the $\left\langle(j)^{2} 0\left|V_{\text {eff }}\right|(j)^{2} 0\right\rangle$ matrix elements have been given. 25,54 Comparison of the decomposition with Eq. (D.25) and Eq. (D.26) allows the extraction of the parameters $\left\langle\mathrm{k}_{\mathrm{v}}>^{2} \theta_{\mathrm{L}} \cdot \mathrm{A}\right.$ knowledge of $\left\langle\mathrm{k}_{\mathrm{v}}\right\rangle$ is required to determine the parameters $<\mathrm{k}_{\mathrm{V}}>\theta_{\mathrm{L}}$ which are needed to calculate 
the transition matrix elements. Following Bohr and Mottelson, $63<\mathrm{k}_{\mathrm{v}}>$ is taken to be $50 \mathrm{MeV}$ in these calculations. Estimates of this quantity, based on reasonable finite potential wells, for various orbitals in several nuclei produce values from roughly $35-75 \mathrm{MeV} .15,16$ Uncertainties in the value of $\left\langle k_{v}\right\rangle$ are probably the major source of error in making this comparison between the spectrum and transitions.

Table 1 gives the values of $<k_{\mathrm{v}}>\theta_{\mathrm{L}}$ deduced in this manner. For $\mathrm{Zr}^{90},<\mathrm{k}_{\mathrm{v}}>\theta_{2}=.119$, which is the same as the value given in Ref. 15 and 16. The latter value was extracted from the effective charge and can be obtained without knowing $\left\langle k_{\mathrm{v}}\right\rangle$. It should be pointed cut that the potential wells used in these references had $\left\langle\mathrm{k}_{\mathrm{v}}>70 \mathrm{MeV}\right.$. In the last column of Table 1 the parameter $C_{L}$ is tabulated. This parameter represents the effective stiffness of the core to $2^{L}$-pole surface vibrations and is simply the inverse of $\theta_{L}$. From the table it is seen. that the core of $\mathrm{Ti}^{50}$ is somewhat softer than the core of $\mathrm{Zr}^{90}$ and the $\mathrm{L}=2$ vibrations are most important in both cases. This is expected as is the indicated increase in core stiffness to higher order vibrations. The indicated core coupling is by no means negligible, however, even for the highest core multipole. Note the large monopole coupling indicated for $2 \mathrm{p}_{1 / 2}$ protons outside the $\mathrm{Sr}^{88}$ core. On the basis of nuclear compressibility, $L=0$ vibrations are not expected to be so important. 
TABLE 1.--Extraction of $<\mathrm{k}_{\mathrm{V}}>\theta_{\mathrm{L}}$ from bound state matrix elements of Kuo and Brown.

\begin{tabular}{|c|c|c|c|c|c|}
\hline \multicolumn{6}{|c|}{$2 r^{90}$} \\
\hline L & $j$ & $<(j)^{2} 0|| G_{3 p-I h}||(j)^{2} 0>(\mathrm{MeV})$ & $\mathrm{M}_{\mathrm{L}}^{\mathrm{O}}$ & $<\mathrm{k}_{\mathrm{V}}>\theta_{\mathrm{L}}$ & $\stackrel{\mathrm{C}_{\mathrm{L}}}{(\mathrm{MeV})}$ \\
\hline 0 & $\lg _{9 / 2}$ & -.020 & .0796 & .00504 & 9920 \\
\hline 2 & $\lg _{9 / 2}$ & -.578 & .0970 & .119 & 420 \\
\hline 4 & $\lg _{9 / 2}$ & -.359 & .0900 & .079 & 633 \\
\hline 6 & $1 g_{9 / 2}$ & -.218 & .0770 & .057 & 877 \\
\hline 8 & $1 g_{9 / 2}$ & -.122 & .542 & .045 & 1110 \\
\hline 0 & $2 p_{1 / 2}$ & -.241 & .0796 & .061 & 820 \\
\hline & & $\mathrm{Ti} 50$ & & & \\
\hline $\mathrm{L}$ & $j$ & $<(j)^{2} 0|| G_{3 p-I h}||(j)^{2} 0>(\mathrm{MeV})$ & $\mathrm{M}_{\mathrm{L}}^{\mathrm{O}}$ & $<\mathrm{k}_{\mathrm{v}}>\theta_{\mathrm{L}}$ & $\stackrel{\mathrm{C}_{\mathrm{L}}}{(\mathrm{MeV})}$ \\
\hline 0 & $\mathrm{f}_{7 / 2}^{\prime}$ & -.033 & .0796 & .00892 & 5610 \\
\hline 2 & $\mathrm{lf}_{7 / 2}$ & -.753 & .0950 & .159 & 314 \\
\hline 4 & ${ } f_{7 / 2}$ & -.460 & .0839 & .110 & 455 \\
\hline 6 & $\operatorname{lf}_{7 / 2}$ & -.233 & .0602 & .0775 & 645 \\
\hline
\end{tabular}


The admixture of a core excited component in a shell model configuration is proportional to $<\mathrm{k}_{\mathrm{V}}>^{2} / \mathrm{C}_{L} \hbar \omega_{L}$ (see Eq. (D. I")). Assuming the hydrodynamical values for the mass parameter $D_{2}$ gives 9.2 and $10.8 \mathrm{MeV}$ for the energies of the effective quadrupole phonon in $\mathrm{Zr}^{90}$ and $\mathrm{Ti}^{50}$. Using these energies and the $\mathrm{C}_{2}$ of Table 1 in Eq. (D.I") leads to values of $12 \%$ and $14 \%$ for the $L=2$ core admixtures In the ground states of $\mathrm{Zr}^{90}$ and $\mathrm{Ti}^{50}$. Admixtures this large are not completely tolerable in view of the perturbative treatment being used. Ref. 15 and 16 report $7 \% \mathrm{~L}=2$ core admixture in the ground stiate of $\mathrm{Zr}^{90}$. The discrepancy cannot be accounted for by differences in. the values of $\left\langle k_{v}\right\rangle$ and $C_{2}$ which have been used here and in those works.

As an example of the pairing effect which is due to the core polarization, the results of shell model calculations of Kuo and Brown for $\mathrm{Ti}^{50}$ and $\mathrm{Zr}^{90}$ are shown in Fig. 1. Theoretical results obtained with and without the inclusion of core polarization are compared with experiment. For both of the spectra shown the zero of energy is that of two non-interacting protons in the lowest available orbit outside of the filled core. The experimental energies have been plotted with the aid of the mass tables of Mattauch et al. 97 The experimental energies for $\mathrm{Ti}^{50}$ have been shifted by $4 \mathrm{MeV}$ 

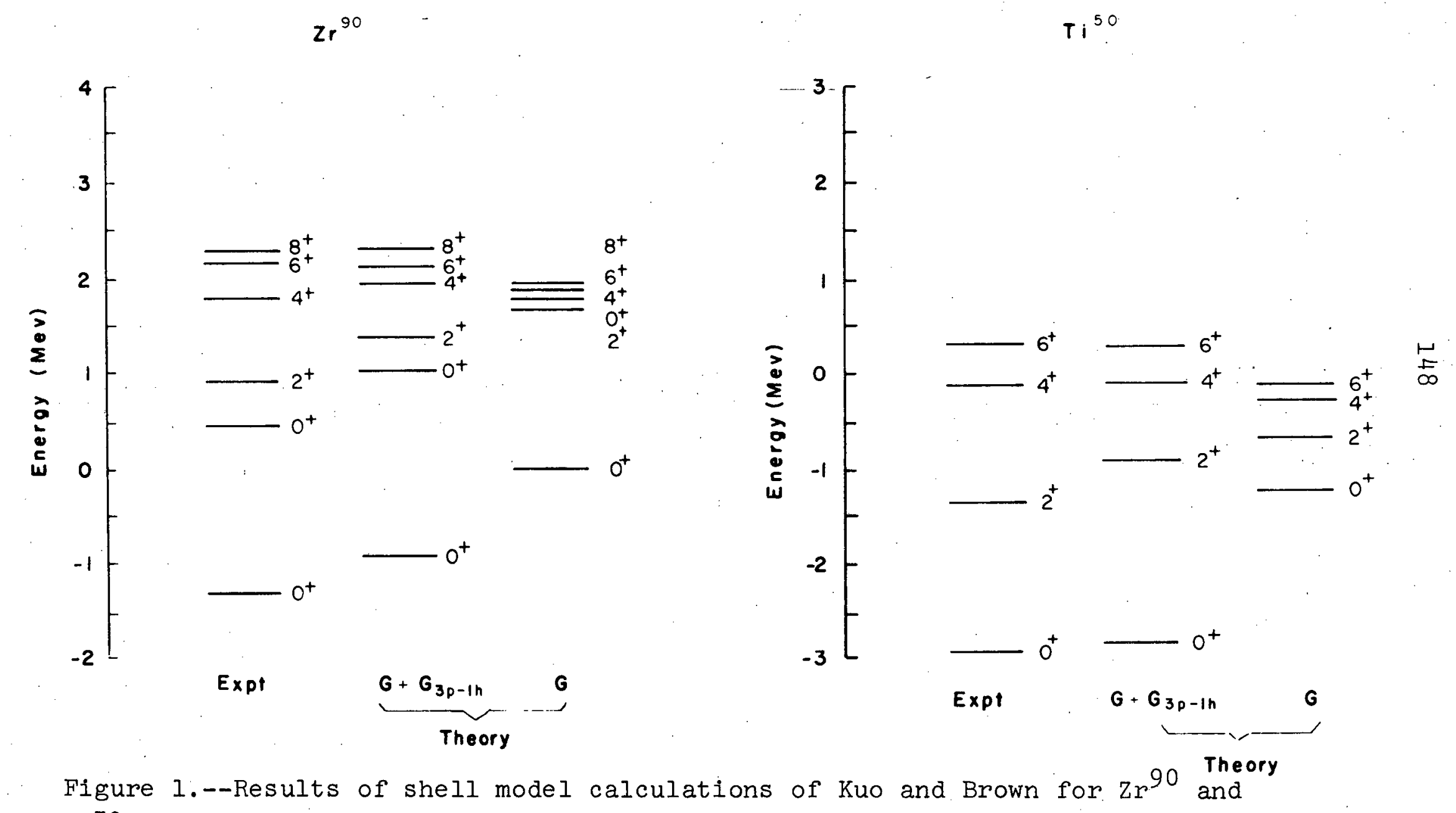

Figure 1.--Results of shell model calculations of Kuo and Brown for $\mathrm{Zr}^{90}$ and $\mathrm{Ti}^{50}$ are compared with experiment. Spectra labeled $G+G_{3 p-I h}$ are results with core polarization included while the label $G$ designates results when core polarIzation is 1gnored. 
because the Coulomb interaction was not included in the shell model matrix elements.

The figure clearly shows that core polarization gives a large attractive contribution to the $J=0$ matrix elements, a small attractive contribution to the $J=2$ matrix elements, and repulsive contributions to matrix elements of higher $\mathrm{J}$. In both cases the theoretical $2^{+}$energy is too high. For $\mathrm{Zr}^{90}$ both of the $\mathrm{O}^{+}$states and the $4+$ state need to be pulled down.

The theoretical results for $\mathrm{TI}^{50}$ are in better agreement with experiment than are those for $\mathrm{Zr}^{90}$. The $\mathrm{Ti}^{50}$ results are for a full $1 f-2 p$ shell calculation while only the $2 \mathrm{p}_{1 / 2}$ and $1 \mathrm{~g}_{9 / 2}$ orbits were included in the $\mathrm{Zr}^{90}$ calculation. Note that the ground state energy in $\mathrm{Ti}^{50}$ is 2.90 MeV below the unperturbed value: $\left\langle\left(\mathrm{If}_{7 / 2}\right)^{2} 0\left|V_{\text {eff }}\right|\left(I f_{7 / 2}\right)^{2} 0\right\rangle$ has the value $-2.068 \mathrm{MeV}$ with -.869 MeV coming from the bare force and $-1.199 \mathrm{MeV}$ as a result of core polarization. The additional - $.832 \mathrm{MeV}$ ground state binding energy is due to very small admixtures (Iess than 5\%) of $\left(1 f_{5 / 2}\right)^{2},\left(2 p_{3 / 2}\right)^{2}$, and $\left(2 \mathrm{p}_{1 / 2}\right)^{2}$ components in the ground state wave function. For $\mathrm{Zr}^{90}$

$$
\begin{aligned}
& <\left(1 g_{9 / 2}\right)^{2} 0\left|\nu_{\text {eff }}\right|\left(1 g_{9 / 2}\right)^{2} 0>=-.57 \text { MeV-1.01 MeV } \\
& <\left(2 \mathrm{p}_{1 / 2}\right)^{2} 0\left|\gamma_{\text {eff }}\right|\left(2 p_{1 / 2}\right)^{2} 0>=-.121 \text { MeV-.0105 MeV }
\end{aligned}
$$


where the first number in each case is the bare matrix element and the second is the $3 p-1 h$ correction. An additional-.3 MeV is added to the first matrix element to account for excitation of the two valence protons to the $\left(\mathrm{Ig}_{7 / 2}\right)^{2}$ configuration and -. $2 \mathrm{MeV}$ is added to the second matrix element to estimate the effect of configurations with two $2 \mathrm{p}_{3 / 2}$ holes. A pure $\left(I f_{7 / 2}\right)^{2}$ calculation for $\mathrm{Ti}^{50}$ would probably also underbind the $0^{+}$ground state.

In summary, the perturbative treatment of core polarization gives a dramatic contribution to the theoretical results; however, the underbinding of the $0^{+}$and $2^{+}$states indicates that the effect is being underestimated. It is uncertain how these deficiencies are distributed between the different core multipoles. Further, the choice $\left\langle\mathrm{k}_{\mathrm{v}}\right\rangle=50 \mathrm{MeV}$ may result in contributions to transitions from core polarization which are somewhat larger than the matrix elements of Kuo and Brown actually imply--at least for $\mathrm{Zr}^{90}$.

Microscopic Transition Densities $\left(\mathrm{Zr}^{90}\right.$ and $\left.\mathrm{Ti}^{50}\right)$

In the completely microscopic calculations for $\mathrm{Zr}^{90}$ and $\mathrm{Ti}^{50}$ (detailed formulae are given in Section 3 of Appendix D) particle-hole pairs have been taken from the following shells:

$\mathrm{Zr}^{90}$ Particles: $2 \mathrm{~d}, \mathrm{lg}_{7 / 2}, 3 \mathrm{~s}, \mathrm{hh}, 2 \mathrm{f}, 3 \mathrm{p}, 1 \mathrm{i}_{13 / 2}, 2 \mathrm{~g}_{9 / 2}$ Holes: $1 d, 2 s, 1 f, 2 p_{3 / 2}$ (and $1 g_{g / 2}, 2 p_{1 / 2}$ for neutrons only)

$\mathrm{Ti} 50$ Particles: $2 \mathrm{p}, 1 \mathrm{f}_{5 / 2}, 1 \mathrm{~g}, 2 \mathrm{~d}, 3 \mathrm{~s}$

Holes: $I p, I d, 2 s$ and $I f_{7 / 2}$ (for neutrons only) 
These orbits include all the particle-hole excitations with energies up to roughly 2 $K$, except those proton-proton hole excitations for which the particle level is the same as the valence orbitals, i.e. In $\mathrm{Zr}^{90}$ the proton particle-hole

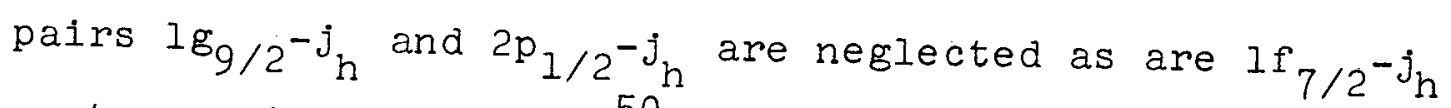
proton excitations in $\mathrm{Ti} 50$. The single particle energy levels have been taken from the Nilsson chart at zero deformation. The parameter hw which fixes both the harmonic oscillator wave functions and the energy denominators has been taken to be 9.1 and $10.5 \mathrm{MeV}$ for $\mathrm{Zr}^{90}$ and $\mathrm{Ti}^{50}$, respectively.

T'he composition of the core transition densities, $\mathrm{F}_{\mathrm{p}}^{\mathrm{LOL}}(\mathrm{C})$ and $\mathrm{F}_{\mathrm{n}}^{\mathrm{LOL}}(\mathrm{C})$, for the $\mathrm{L}=2-8$ transitions in $\mathrm{zr}^{90}$ and the $\mathrm{L}=2-6$ transitions in $\mathrm{Ti}^{50}$ are displayed in Tables 2 and 3. The important particle-hole pairs are listed with their energy denominators. The amplitude of the state $\left|\left[(j j) L\left(j_{p} \bar{j}_{h}\right) L\right] 0\right\rangle$ in the $\mid\left(j^{2}\right) 0>$ ground state, $A_{G}$, and the amplitude of the state $\left|\left[(j j) O\left(j_{p} \bar{j}_{h}\right) L\right] L\right\rangle$ in the $\left|(j)^{2} L\right\rangle$ excited state, $A_{E}$, are listed along with the fractional contributions, $\%$, of a particular particle-hole excitation to its respective core transition density (either proton or neutron). Observe that in $\mathrm{Zr}^{90}$ it is only the states with $j=1 \mathrm{~g}_{9 / 2}$ that are involved in the $\mathrm{L}=2-8$ transitions. For the definition of the amplitudes see Eq. (D.39). The ratio of $\mathrm{F}_{\mathrm{n}}^{\mathrm{LOL}}(\mathrm{C})$ to $\mathrm{F}_{\mathrm{p}}^{\mathrm{LOL}}(\mathrm{C})$ is also given in each case--denoted by $\mathrm{N} / \mathrm{P}$. 
TABLE 2.--Composition of core transition densities for $L=2-8$ transitions in $\mathrm{Zr}^{90}$.

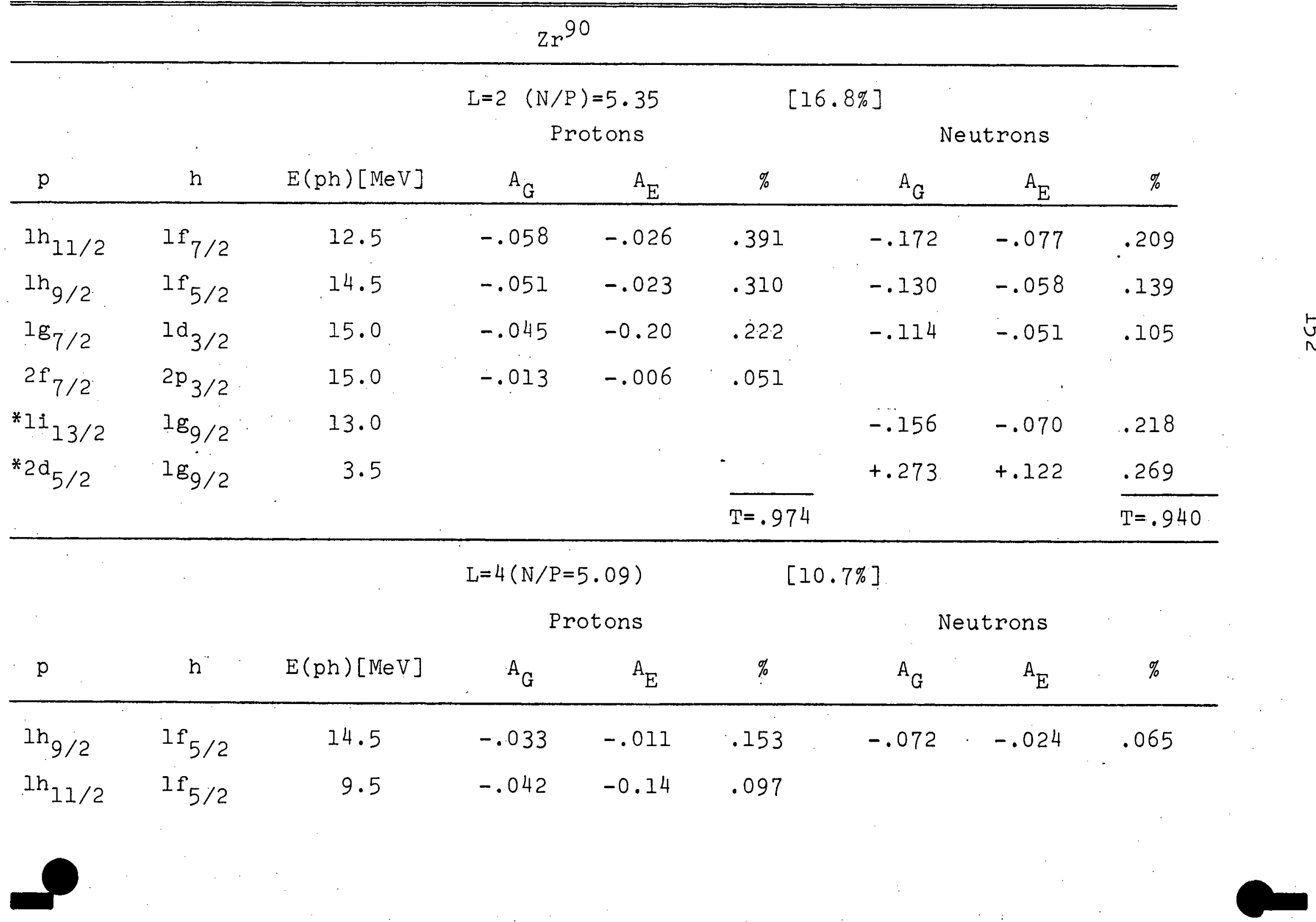


TAELE 2.--Continued

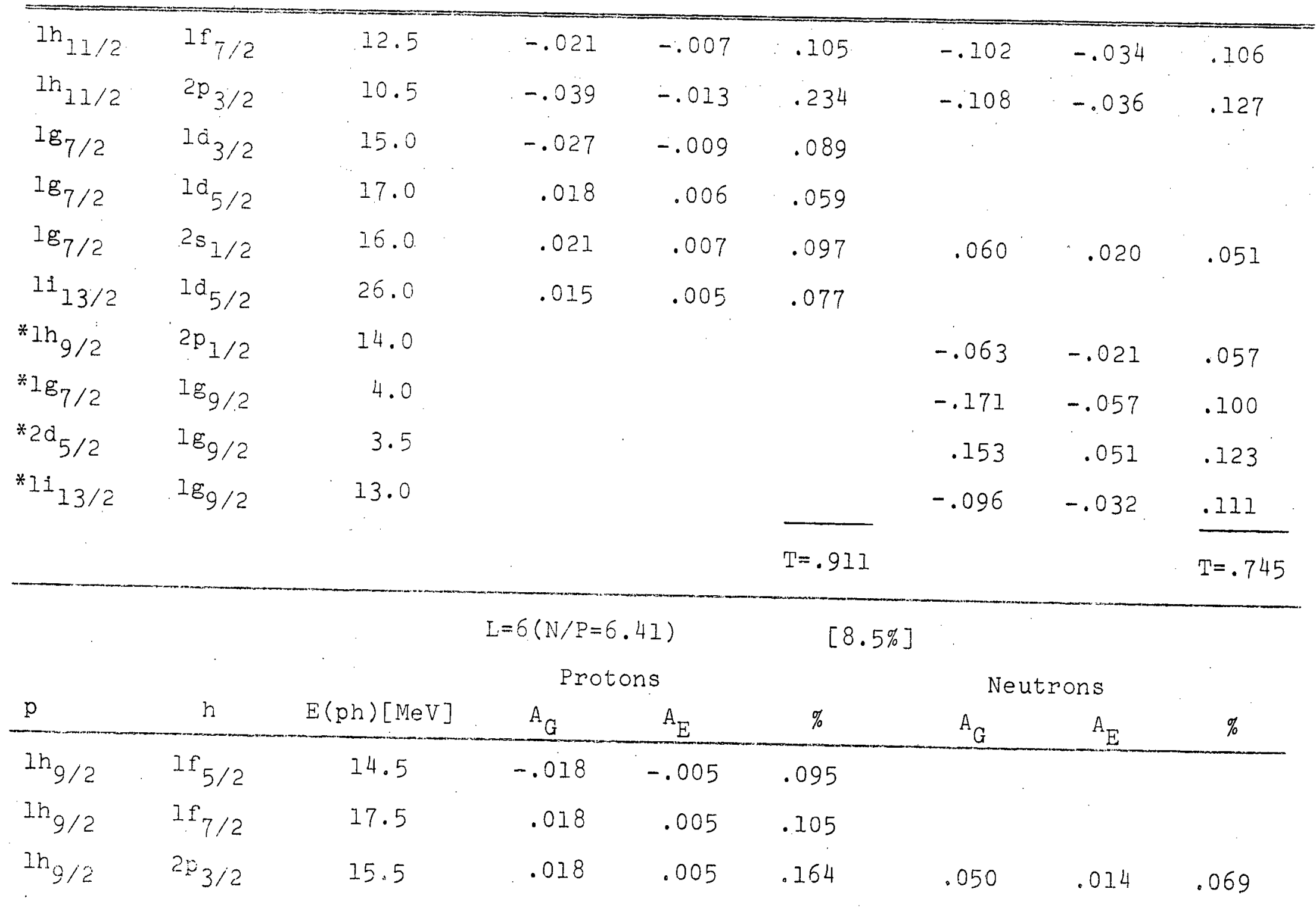


TABLE 2.--Continued.

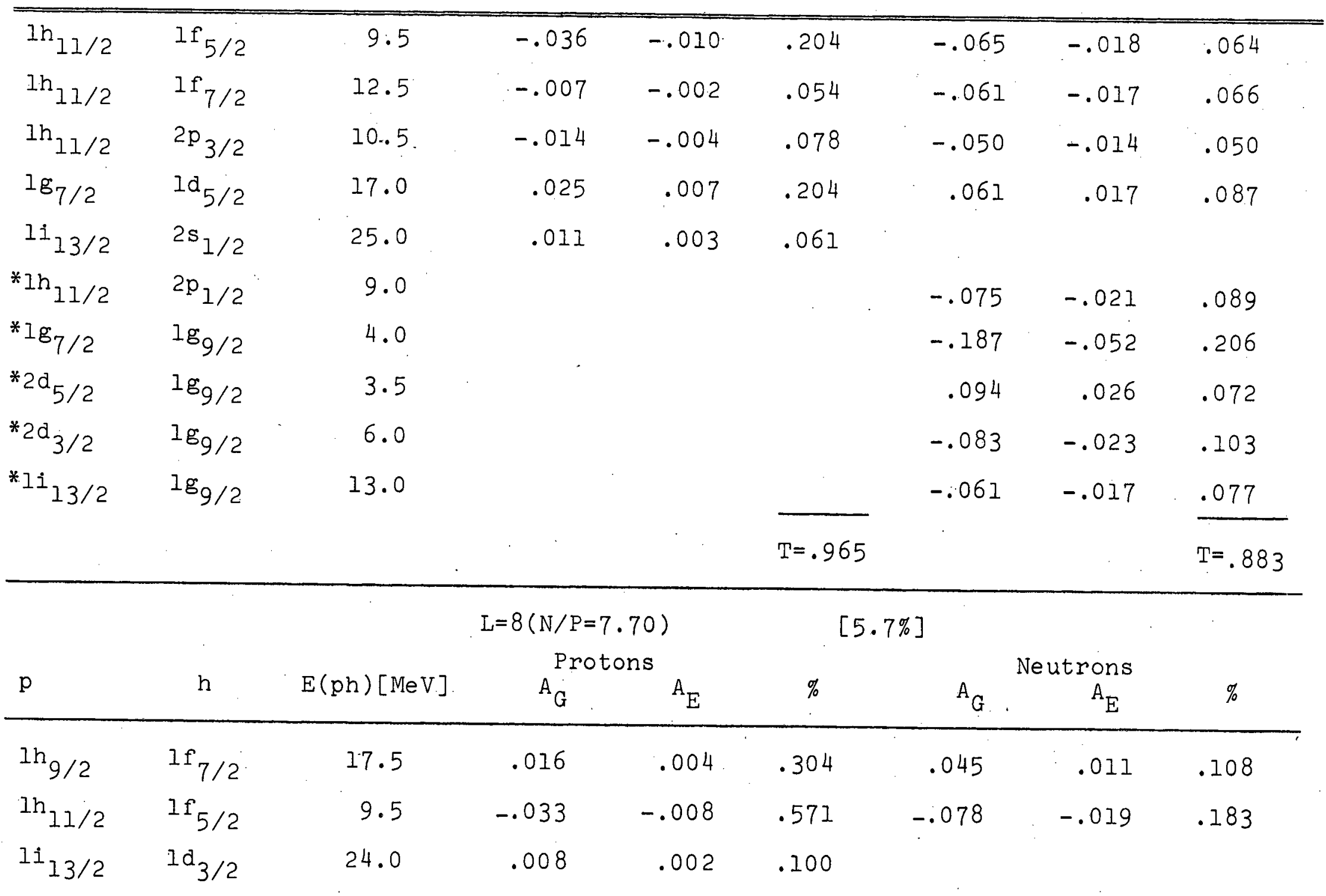


TABLE'2.--Continued .

\begin{tabular}{|c|c|c|c|c|c|c|}
\hline${ }^{*}{ }^{18} 8_{7 / 2}$ & $1 g_{9 / 2}$ & 4.0 & & -.214 & -.052 & .564 \\
\hline \multirow[t]{2}{*}{${ }^{*}{ }^{1} i_{13 / 2}$} & $1 g_{9 / 2}$ & 13.0 & & -.037 & -.009 & .056 \\
\hline & & & $\mathrm{T}=.975$ & & & $\mathrm{~T}=.911$ \\
\hline
\end{tabular}


TABLE 3.--Composition of core transition densities for $L=2-6$ transitions in $T_{1} 50$.

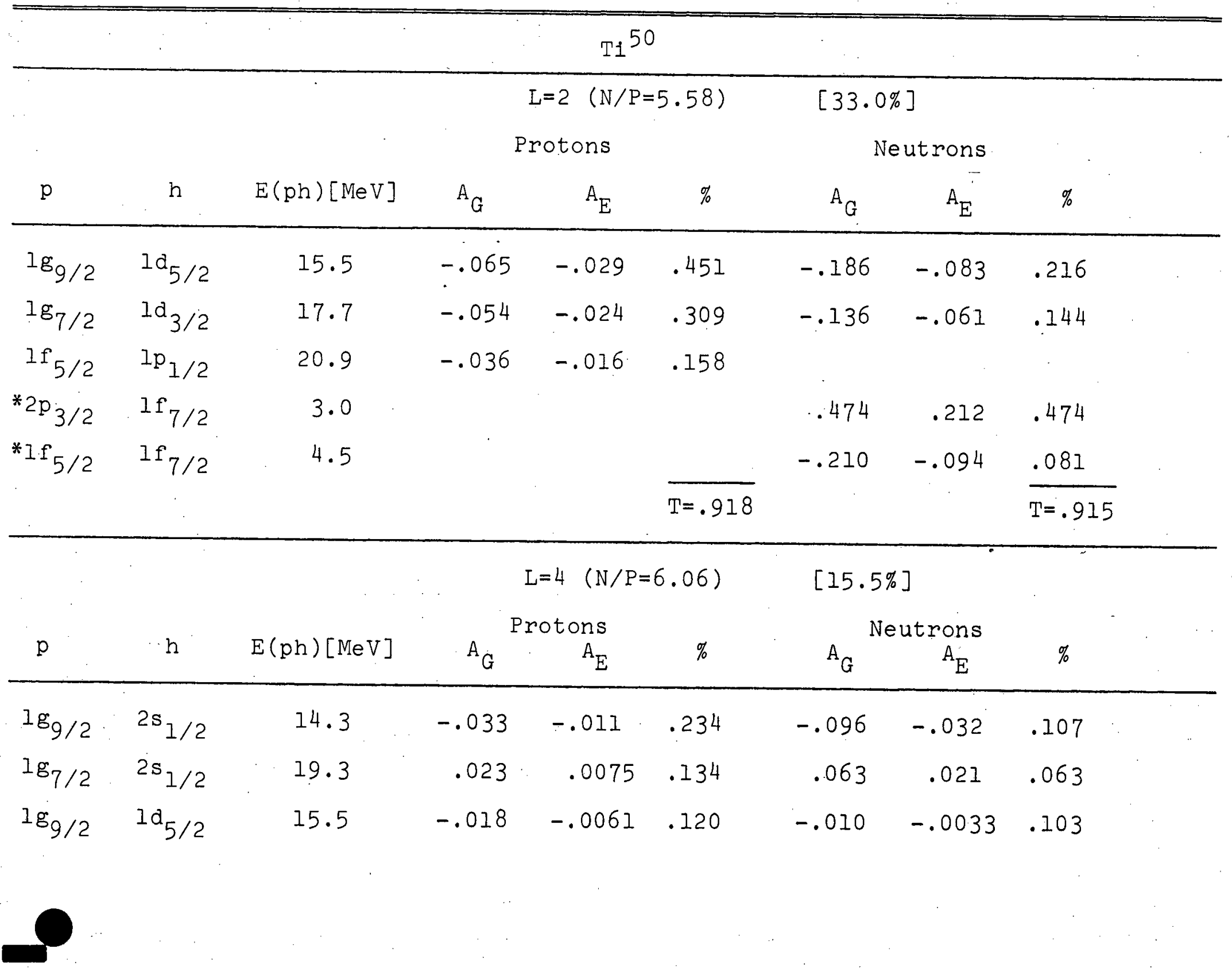


TABLE 3.--Continued.

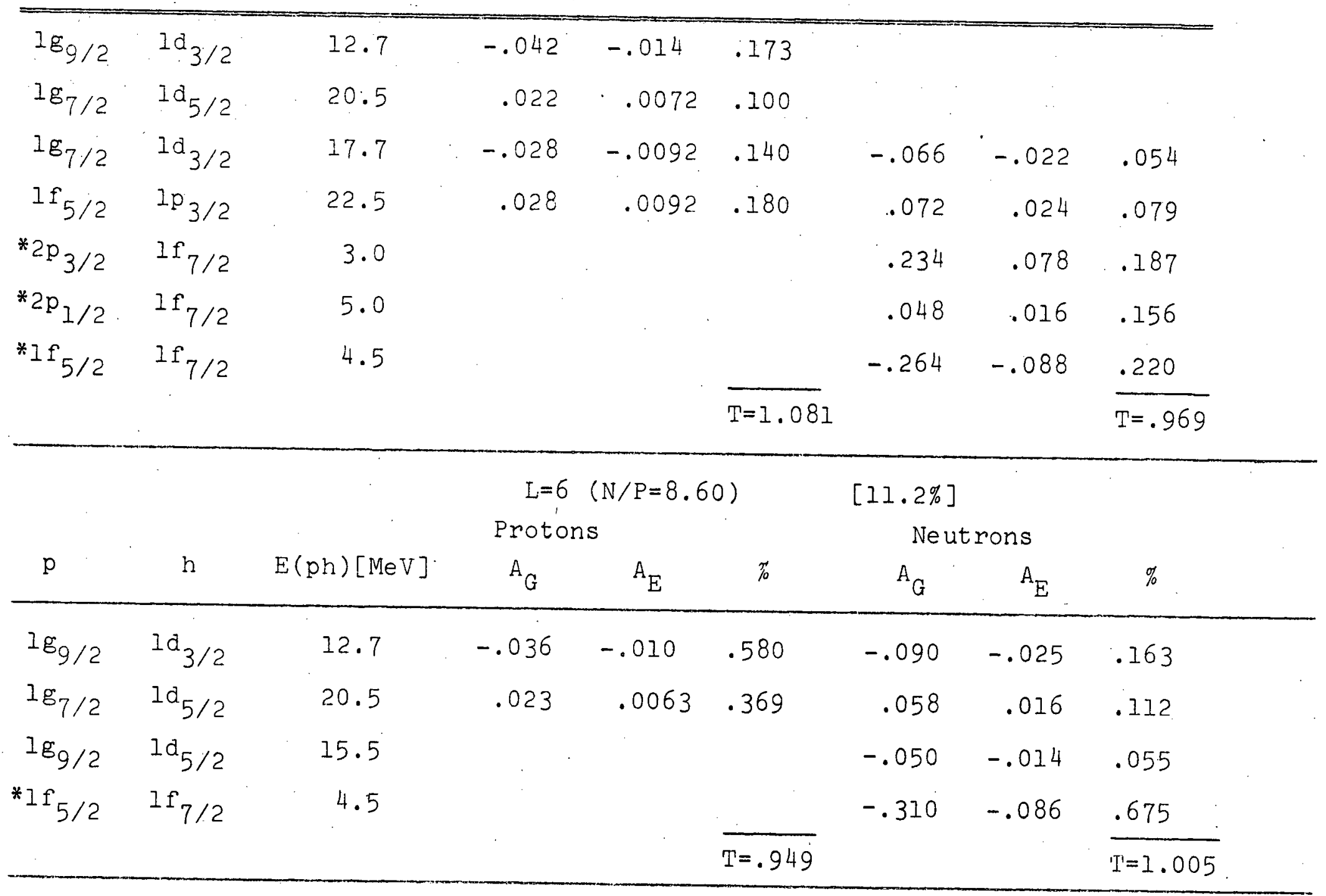


The transition densities are, of course, functions of radial position within the target nucleus. The radial dependence of the valence transition density, $F_{p}^{L O L}(D)$, is given by $u_{n \ell}(r) u_{n \ell}(r)$ while the radial dependence of the contribution of a particular particle-hole excitation to its core transition density is given by $u_{n_{p} \ell_{p}}(r) u_{n_{h} \ell_{h}}(r)$. The particle-hole excitations which give important contributions almost invariably satisfy the condition

$$
u_{n_{p} \ell_{p}}(r) u_{n_{h} \ell_{h}}(r) \sim u_{n \ell}(r) u_{n \ell}(r)
$$

i.e. have radial wave functions similar in shape to those of the active valence nucleons. This fact was expected and used to fix the sign the radial integrals in arguing the phase of the effect of core polarization on transitions in Section 3 of Appendix D. Exceptions occur, for the most part, only when a particularly small energy denominator is involved. Since the radial wave functions of the valence nucleons are nodeless for these cases it is not surprising that most of the important particle-hole excitations are formed from orbitals with nodeless radial wave functions.

The essential point is that $\mathrm{F}_{\mathrm{p}}^{\mathrm{LOL}}(\mathrm{D}), \mathrm{F}_{\mathrm{p}}^{\mathrm{LOL}}(\mathrm{C}), \mathrm{F}_{\mathrm{n}}^{\mathrm{LOL}}(\mathrm{C})$, and their important individual components have the same sign and approximately the same radial shape. This fact, which is a result of direct calculation, was assumed in deriving the empirical formula for enhancement factors due to core polarization in Section 4 of Appendix D. It also justifies 
comparison of the transition densities and their components through percentages and ratios as is done in Table 2 and 3 .

In the tables only those particle-hole excitations which make up at least $5 \%$ of their respective transition densities are listed. In each case the total fraction of the complete core transition density due to the listed particle-hole excitations is given. This is designated by $T$. This number illustrates the importance of contributions not included in the tables.

Relatively few particle-hole pairs make important contributions to the transition densities, particularly for the $L=2$ transitions, the $L=6$ transition in $T i^{50}$ and the $L=8$ transition in $\mathrm{Zr}^{90}$. It is also noted that for the $\mathrm{L}=2$ transitions the following condition is highly favored.

$$
j_{p}=l_{p} \pm \frac{1}{2} \quad j_{h}=l_{h} \pm \frac{1}{2} \quad\left|j_{p}-j_{h}\right|=L=2
$$

This was also noted by Zamick and Federman in their calculations of quadrupole effective charges. ${ }^{88}$ For the $\mathrm{L}=6$ transition in $\mathrm{Ti}^{50}$ and the $\mathrm{L}=8$ transition in $\mathrm{zr}^{90}$ a similar condition is favored, namely:

$$
j_{p}=l_{p} \pm \frac{1}{2} \quad j_{h}=l_{h} \mp \frac{1}{2} \quad j_{p}+j_{h}=L=6 \text { or } 8
$$

These results follow from Eq. (D.52) and Eq. (D.49) which show that the contribution of a particular particle-hole pair to the transition density is proportional to $\left(2 j_{p}+1\right) x$ $\left[\mathbb{M}_{\tau_{p} \tau_{h}}^{L O L}\left(j_{h} j_{p}\right)\right]^{2}$ which is essentially given by 


$$
\left(2 j_{p}+1\right)\left(2 j_{h}+1\right)\left(\begin{array}{ccc}
j_{p} & j_{h} & L \\
l / 2 & -1 / 2 & 0
\end{array}\right)^{2} \text {. }
$$

It can be shown that vector addition coefficients of the above type achieve their maximum value when the conditions cited above are fulfilled. 63

The increased fractionization of the core polarization strength for the transitions with intermediate L-transfers occurs because the above coupling conditions are not satisfied simultaneously with the condition that the particle and hole orbitals have nodeless radial wave functions, i.e. particle-hole excitations with nodeless radial wave functions that do not satisfy the coupling condition are as favorable as those satisfying the reversed conditions. The percentages given in the brackets for each transition are the admixture of particle-hole pairs coupled to $L$ in the ground state. This is obtained by summing the squares of the $A_{G}$. There are $16.8 \% \mathrm{~L}=2$ particle-hole pairs in the $\left(\lg _{9 / 2}\right)^{2}$ component of the ground state of $\mathrm{Zr}^{90}$. The $\mathrm{L}=2$ admixture in the ground state of $\mathrm{Ti}^{50}$ is $33 \%$. These values are to be compared with the corresponding values of $12 \%$ and $14 \%$ obtained using the macroscopic vibrational model to describe the core. The comparison is relative as the energy denominators used in obtaining the latter values are somewhat arbitrary .

The core transition densities which have been obtained here would be essentially unchanged if average energy denominators of 14.5 and $17.1 \mathrm{MeV}$ were used in place of 
the Nilsson denominators for $\mathrm{Zr}^{90}$ and $\mathrm{Ti}^{50}$, respectively, in all instances except when the particle and hole occupy sub-levels of the same principle shell. These average energy denominators are somewhat smaller than the values zh $\omega$ assumed by Kuo and Brown. ${ }^{25,54}$ The Nilsson scheme gives small energy denominators when the particle and hole are in the same principle shell. This is consistent with Kuo and Brown's use of empirical energy differences for these cases. Because of their smallness, it is these energy denominators which are most uncertain. Further it is evident that the transition densities are very sensitive to these small energy denominators since the $2 \mathrm{~d}_{5 / 2}-1 \mathrm{~g}_{9 / 2}$ and $1 g_{7 / 2^{-}}$ $1 \mathrm{~g}_{9 / 2}$ neutron-neutron hole pairs in $2 \mathrm{r}^{90}$ and the $2 \mathrm{p}_{3 / 2^{-}}$ $I f_{7 / 2}$ and $l f_{5 / 2}-1 f_{7 / 2}$ neutron-neutron hole pairs in $\mathrm{Ti}^{50}$ (all of which have small energy denominators) appear in the wave functions with fairly large amplitudes. It is estimated that a factor of two change in these small denominators could make $20-40 \%$ changes in the magnitude of the transition densities obtained, with the core transition densities in $\mathrm{Ti}^{50}$ being slightly more sensitive to this change than those for $\mathrm{Zr}^{90}$.

For $\mathrm{Ti}^{50}$, the differences between the $\mathrm{L}=2$ ground state core admixtures obtained in the microscopic calculations as compared to those obtained in the macroscopic parameterization are attributable to differences in the energy denominators used here and in Ref. 25. Most notably, the latter quotes larger values for the small energy denominators in 
$\mathrm{Ti}^{50}$ than have been used in this work. Specifically it gives $E(p h)=4.8,6.82$, and $8.75 \mathrm{MeV}$. for the $2 \mathrm{p}_{3 / 2}-1 \mathrm{f}_{7 / 2}, 2 \mathrm{p}_{1 / 2^{-}}$ $l f_{7 / 2}$, and $l f_{5 / 2}-1 f_{7 / 2}$ neutron-neutron hole excitations, respectively. Corresponding values used in this work are $3.0,5.0$, and $4.5 \mathrm{MeV}$. Replacing the smaller energy denominators by the larger ones reduces the $\mathrm{L}=2$ ground state admixtures in $\mathrm{Ti}^{50}$ from $33 \%$ to $18 \%$ and a $20 \%$ decrease in the magnitude of the corresponding neutron core transition density.

Probably the most startling feature of the results presented in Tables 2 and 3 is the large imbalance between the proton-proton hole and neutron-neutron hole core polarization contributions. The difference is so large as to seem unreasonable. It is the natural result of these calculations for three reasons. The first is simply the difference in strength between the neutron-proton and protonproton forces which results in an average increase of about 2.75 in the importance of a particular neutron-neutron hole as compared with the corresponding proton-proton hole. The second is the presence of the excess core neutrons which contribute neutron-neutron holes via small energy denominators. From 45-70\% of the neutron core transition densities result from excess neutrons. Such contributions are indicated by an asterick in the tables. The last reason is the neglect of the proton particle-hole pairs for which the particle level is that of the valence protons. Federman and Zamick $^{8} 8$ included such contributions in their investigation of quadrupole transition rates and found that they 
gave roughly $35 \%$ of the core polarization due to protons. They observed a neutron-proton imbalance in their results, but they considered evenly closed cores so it was due only to the $n-p$ and $p-p$ forces difference.

$\underline{I}=0$ Transition in $\mathrm{Zr}^{90}$

The $\mathrm{L}=0$ transition in $\mathrm{Zr}^{90}$ needs separate discussion. As was mentioned previously the ground state wave function and $0^{+}(Q=-1.75 \mathrm{MeV})$ wave function are mutually orthogonal combinations of the $\left(1 g_{9 / 2}\right)^{2}$ and $\left(2 p_{1 / 2}\right)^{2}$ configurations, i.e.

$$
\begin{aligned}
& \mid 0^{+}(\text {g.s. })>=.6\left|\left(\lg _{9 / 2}\right)^{2} 0>-.8\right|\left(2 \mathrm{p}_{1 / 2}\right)^{2} 0> \\
& 10^{+}(\mathrm{Q}=-1.75 \mathrm{MeV})>=.8\left|\left(\lg _{9 / 2}\right)^{2} 0>+.6\right|\left(2 \mathrm{p}_{1 / 2}\right)^{2} 0>
\end{aligned}
$$

The transition density has two components--a $\left(\lg _{9 / 2}\right)^{2}$ component and a $\left(2 \mathrm{p}_{1 / 2}\right)^{2}$ component corresponding to the matrix elements

$$
\begin{gathered}
.48<\left(\lg _{9 / 2}\right)^{2}|\mathrm{~T}|\left(1 \mathrm{~g}_{9 / 2}\right)^{2}> \\
-.48<\left(2 \mathrm{p}_{1 / 2}\right)^{2} 0|\mathrm{~T}|\left(2 \mathrm{p}_{1 / 2}\right)^{2}>
\end{gathered}
$$

Strictly speaking the theory also allows for contributions corresponding to the matrix elements:

$$
\cdot 36<\left(2 \mathrm{p}_{1 / 2}\right)_{0}^{2}|\mathrm{~T}|\left(1 \mathrm{~g}_{9 / 2}\right)^{2} 0>
$$




$$
-.64<\left(\lg _{9 / 2}\right)^{2} 0|T|\left(2 p_{1 / 2}\right)^{2} 0>
$$

There is no valence contribution to these matrix elements as the initial and final valence configurations differ in the state of more than one particle. Further the $3 \mathrm{p}$-lh intermediate states which can contribute must have two protons in the $\lg _{9 / 2}\left(2 \mathrm{p}_{1 / 2}\right)$ orbit and a third proton in the $2 \mathrm{p}_{1 / 2}$ $\left(\mathrm{lg}_{9 / 2}\right)$ orbit--all coupled to a proton hole. These are neglected. Similar contributions, corresponding to the matrix elements

$$
-.8<\left(\lg _{9 / 2}\right)^{2} \mathrm{~L}|\mathrm{~T}|\left(2 \mathrm{p}_{1 / 2}\right)^{2} 0>
$$

have been neglected in treating the other transitions in $\mathrm{Zr}^{90}$.

The structure of the transition density for the $L=0$ transition is illustrated in Fig. 2. Shown at the top are $\mathrm{F}_{\mathrm{p}}^{\mathrm{OOO}}(\mathrm{D})[D], \mathrm{F}_{\mathrm{p}}^{\mathrm{OOO}}(\mathrm{C})[\mathrm{p}-\overline{\mathrm{p}}], \mathrm{F}_{\mathrm{p}}^{\mathrm{OOO}}(\mathrm{T})=\mathrm{F}_{\mathrm{p}}^{\mathrm{OOO}}(\mathrm{D})+\mathrm{F}_{\mathrm{p}}^{\mathrm{OOO}}(\mathrm{C})[\mathrm{D}+\mathrm{p}-\overline{\mathrm{p}}]$, and $\mathrm{F}_{\mathrm{n}}^{\mathrm{OOO}}(\mathrm{T})=\mathrm{F}_{\mathrm{n}}^{\mathrm{OOO}}(\mathrm{C})[\mathrm{n}-\overline{\mathrm{n}}]$ for the $\left(\lg _{g / 2}\right)^{2}$ configuration. Corresponding information for the $\left(2 p_{1 / 2}\right)^{2}$ configuration is shown in the middle. The complete valence transition density $[D]$, the complete proton transition density [P], and the complete neutron transition density [N] are shown at the bottom. Here [D] is the sum of the two curves labeled [D] in the top two drawings, [P] is the sum of the curves labeled $[D+p-\bar{p}]$, and $[N]$ is the sum of the curves labeled $[n-\bar{n}]$. 
TRANSITION DENSITY

$\mathrm{Zr}=\mathrm{p}$

$O^{+}(Q=-1.75 \mathrm{Mev})$

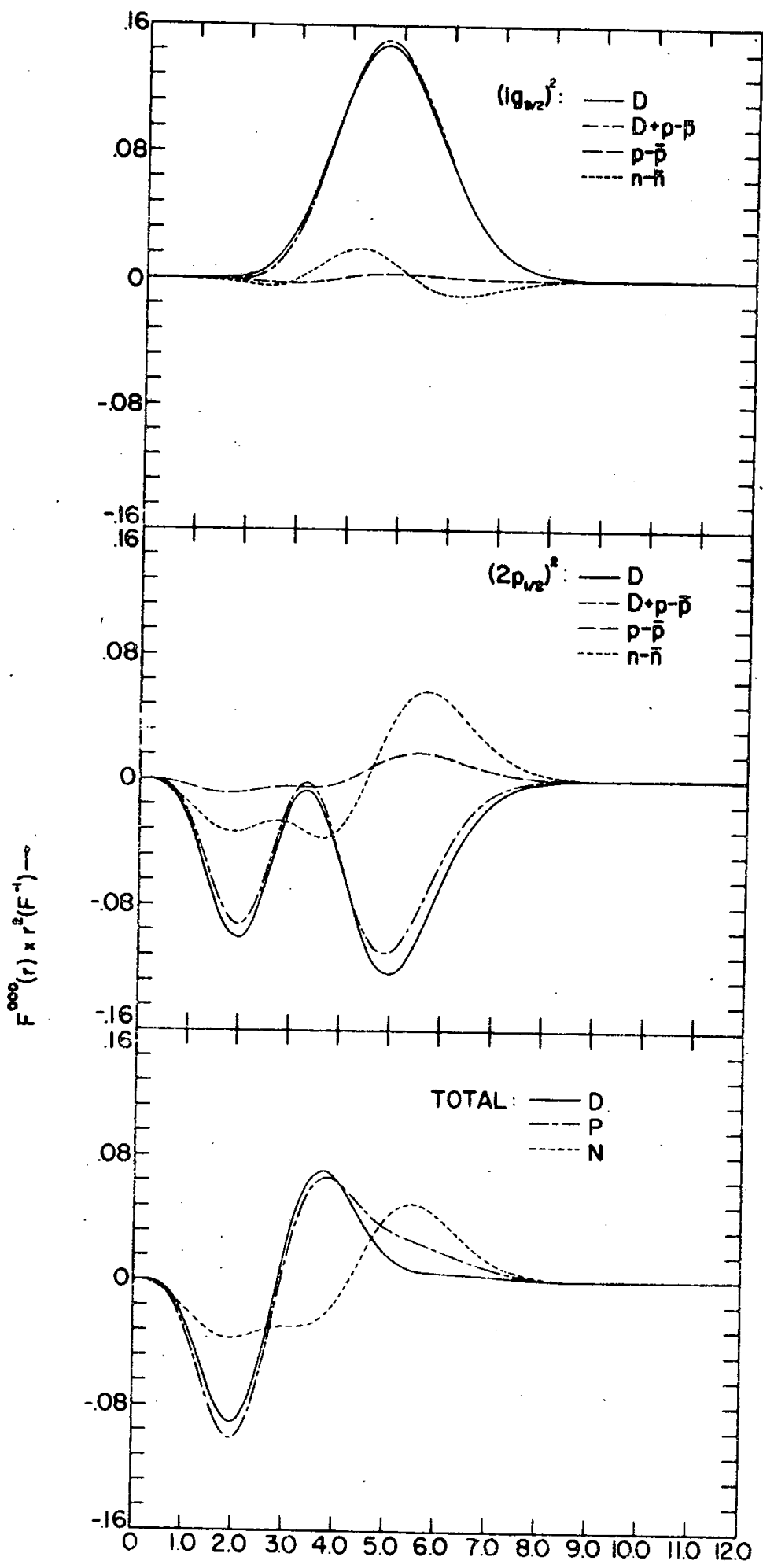

$r(F) \rightarrow$

Figure 2.--Structure of transition density for $L=0$ transition in $2 r^{90}$. 
As in the previous cases, neutron core excitation are found to be more important than proton core excitations. The complete proton transition density does not differ appreciably from the complete valence transition density. The interior minimum of. $\mathrm{F}_{\mathrm{p}}^{\mathrm{OOO}}$ (D) has been increased, the surface maximum has been decreased and shifted slightly outward, and a longer tail appears as a result of $\mathrm{F}_{\mathrm{p}}^{000}(\mathrm{C})$. The core transition densities are oscillatory and are not too similar to the valence transition densitities. Only particle-hole pairs with $j_{p}=j_{h}$ contribute to the core transition densities. As the available particle and hole levels with the same total angular momentum do not have the same principle quantum number, the oscillatory shape results. The small core transition densities for the $\left(\lg _{9 / 2}\right)^{2}$ configuration is understood in terms of the poor overlap of $u_{14}(r) u_{14}(r)$ with $u_{n_{p} \ell_{p}}(r) u_{n_{h} \ell_{h}}(r)$ when $n_{p} \neq n_{h}$. The overlap of $u_{2 l}(r) u_{2 l}(r)$ with $u_{n_{p} l_{p}}(r) u_{n_{h} l_{h}}(r)\left(n_{p} \neq n_{h}\right)$ is better, which explains the larger core transition densities obtained for the $\left(2 \mathrm{p}_{1 / 2}\right)^{2}$ configuration. In the latter case, the radial integrals (see Section 3 of Appendix D) still have the sign of the two-body force and the difference in sign between the core transition densities and the valence transition density for large $r$ is just the difference between $u_{21}(r) u_{21}(r)$ and $u_{n_{p} l_{p}}(r) u_{n_{h} l_{h}}(r)\left(n_{p} \neq n_{h}\right)$ at large $r$. The net effect of core polarization will be an enhancement of this transition, although it occurs as a result of inhibition of the $\left(2 p_{1 / 2}\right)^{2}$ contribution to the transition. 
All particle-hole pairs which contribute to this transition are listed in Table 4 with their energy denominators and the amplitudes $A_{G} \cdot A_{E}$ is not given since it is equal to $A_{G}$ for this case. Percentage contributions are not given either since differences in radial shape between the various components do not allow such a comparison. Ground state admixtures are given in brackets as before. These are quite small. Excitations involving excess core neutrons are indicated with an asterik. They do not contribute to this transition via small energy denominators and thus do not play a special role in this case.

Microscopic Transition Density for Transition
to $Q=-.908$ MeV State of Y 89

The excitation of the $Q=-.908 \mathrm{MeV}$ level of $\mathrm{Y}^{89}$ for incident protons of 18.9, 24.5, and $61.2 \mathrm{MeV}$ is considered. In the ground state of this nucleus the valence proton is in the $2 \mathrm{p}_{1 / 2}$ shell and for the excited state being considered it is in the $1 g_{9 / 2}$ orbit. The triads (LSJ) which can contribute to this transition are (314), (514), (505), and (515). None of these are forbldden in the simple shell model interpretation of this transition so there is no breaking of the valence transition selection rules because of core polarization. It is found that the contributions from (514) and (515) are small enough to be neglected. The microscopic transition densities for the (314) and (505) triads have been calculated by taking particle-hole pairs from the following levels: 
TABLE 4.--Composition of core transition densities for $\mathrm{L}=0$ transition in $\mathrm{Zr} 90$.

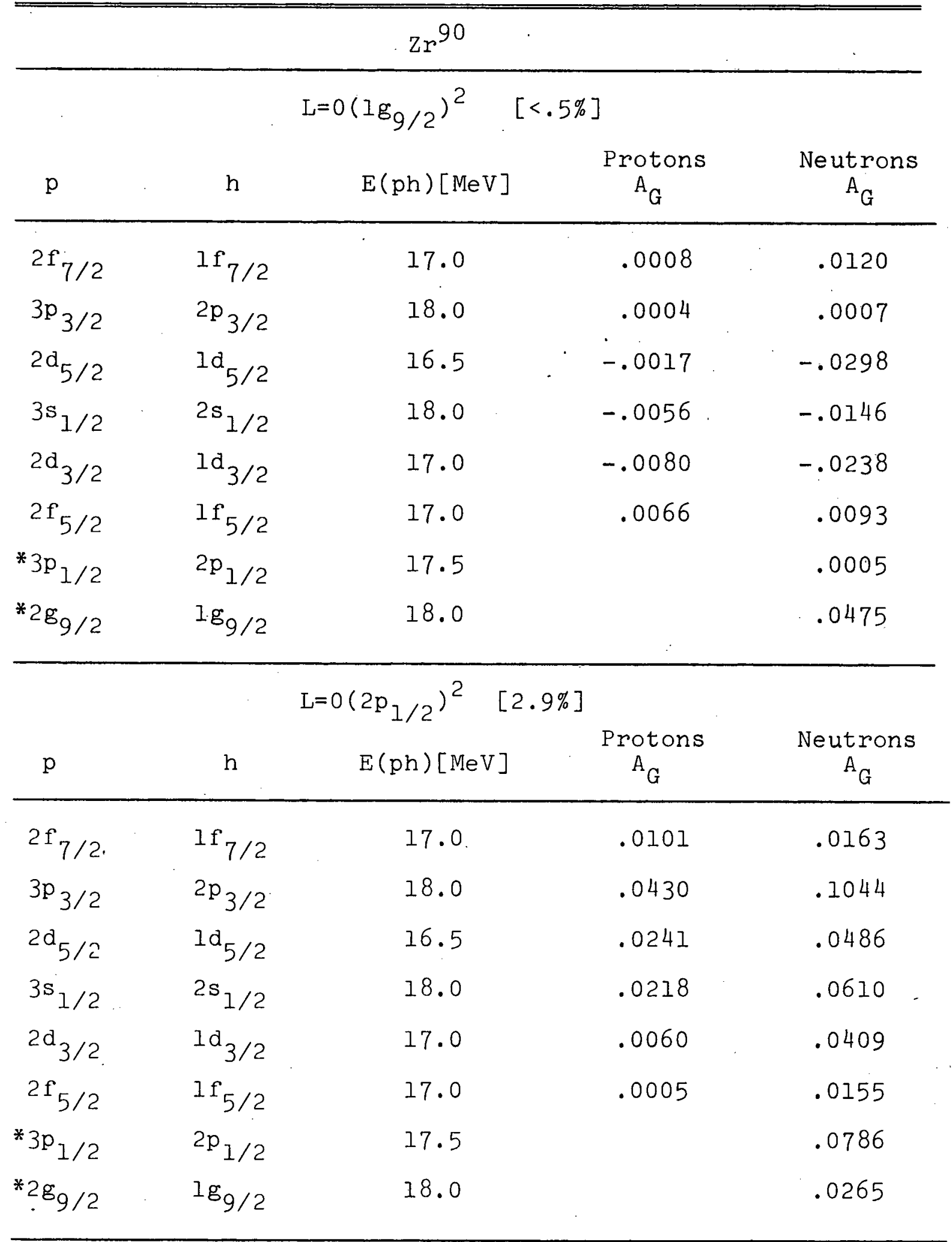


Particles: $2 \mathrm{~d}, 1 \mathrm{~g}_{7 / 2}, 3 \mathrm{~s}$, Ih, $2 \mathrm{f}, 3 \mathrm{p}, \mathrm{li}_{13 / 2}, 2 \mathrm{~g}_{9 / 2}$ Holes: If, $2 p_{3 / 2}$ (and $2 p_{1 / 2}, \lg _{9 / 2}$ for neutrons only) Note that this transition involves a change in parity--thus the particle-hole pairs contributing to the core polarization here are not the same as those involved in the $\mathrm{zr}^{90}$ transitions which have been considered. The orbits listed include all the particle-hole pairs with energies up to roughly lkw with

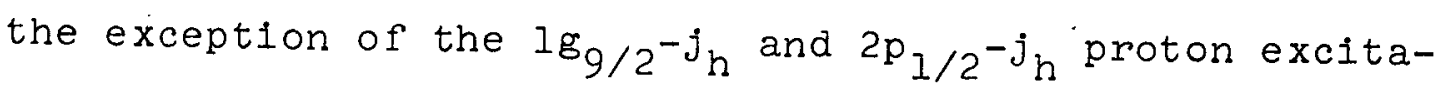
tions. By including the $2 g_{9 / 2}$ and $l_{13 / 2}$ particle levels a few 2 hw excitations are brought in. The constant hw has been fixed at $9.1 \mathrm{MeV}$ for this case--the same as for $\mathrm{Zr}^{90}$. The composition of $\mathrm{F}_{\mathrm{p}}^{314}(\mathrm{C}), \mathrm{F}_{\mathrm{n}}^{314}(\mathrm{C}), \mathrm{F}_{\mathrm{p}}^{505}(\mathrm{C})$, and $\mathrm{F}_{\mathrm{n}}^{505}$ (C) is given in Table 5. The format of this table is the same as that of Tables 2 and $3 . A_{E}$ is the amplitude of the state $\mid 2 p_{1 / 2}\left(j_{p} \bar{j}_{h}\right) J ; 9 / 2>$ in the $\mid 1 g_{9 / 2}>$ excited state and $A_{G}$ is the amplitude of the state $\mid l_{9 / 2}\left(j_{p} \bar{j}_{h}\right) J ; I / 2>$ in the $\left|2 p_{1 / 2}\right\rangle$ ground state. For the expression for calculating these amplitudes see Eq. (D.33). The $J=4$ ground state admixtures are almost zero while the $J=5$ ground state admixtures are just slightly smaller than the $\mathrm{L}=6$ ground state admixtures which were obtained for $2 \mathrm{r}^{90}$.

$\mathrm{F}_{\mathrm{p}}^{314}$ (C) is larger than $\mathrm{F}_{\mathrm{n}}^{314}$ (C) as is indicated by the $\mathrm{N} / \mathrm{P}$ ratio of -.383 . The minus sign indicates that $\mathrm{F}_{\mathrm{p}}^{314}$ (C) is opposite in sign to $\mathrm{F}_{\mathrm{p}}^{314}$ (D) while $\mathrm{F}_{\mathrm{n}}^{314}$ (C) has the same sign as $\mathrm{F}_{\mathrm{p}}^{314}(\mathrm{D})$. The sign difference is a result of the 
TABLE 5.--Composition of core transition densities for transition to Q $=-.908 \mathrm{MeV}$ level of $\mathrm{Y}^{89}$.

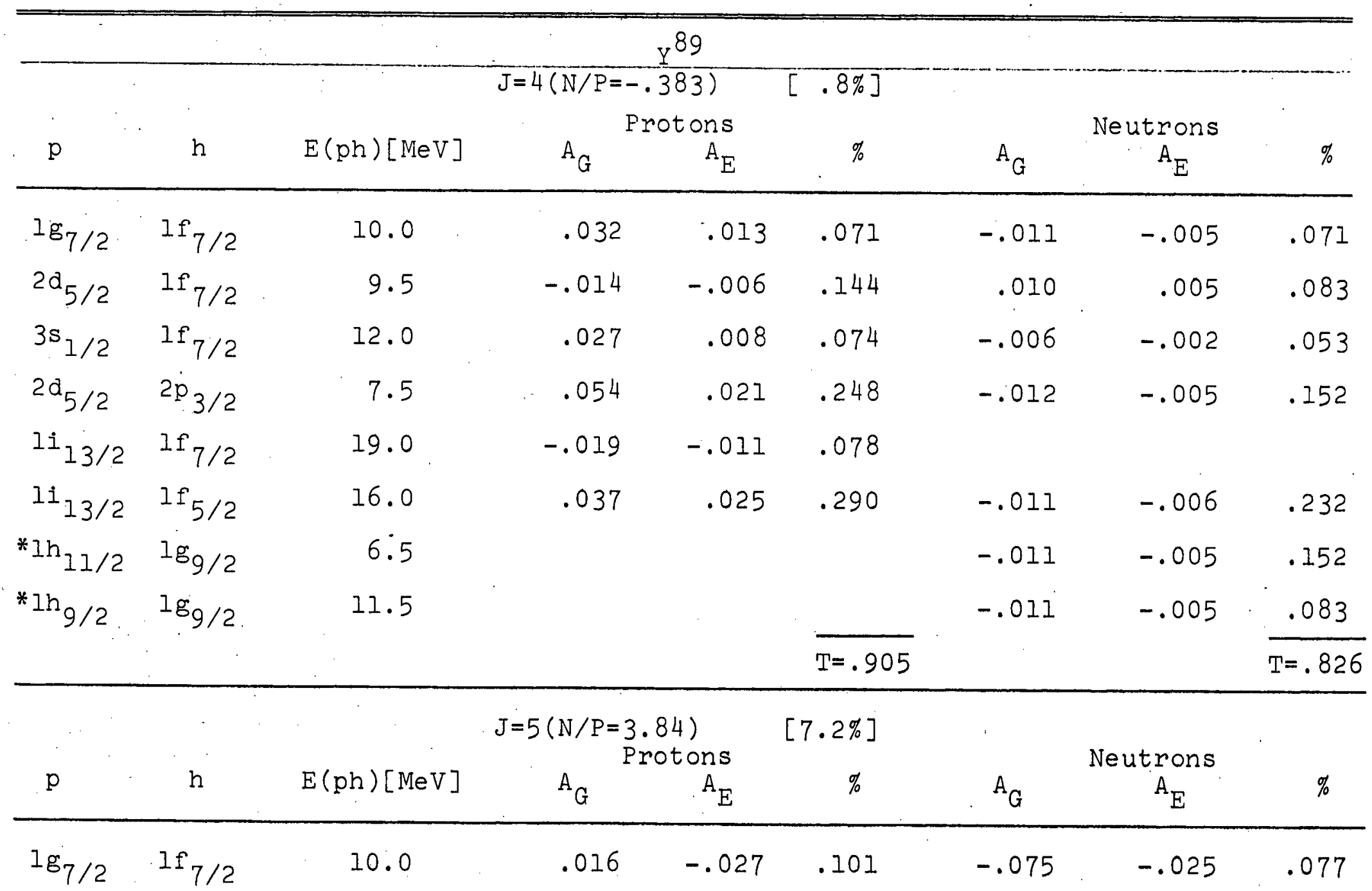


TABLE 5.--Continued

\begin{tabular}{|c|c|c|c|c|c|c|c|c|}
\hline $\lg _{7 / 2}$ & $\operatorname{lf}_{5 / 2}$ & 7.0 & .024 & .017 & .128 & .088 & .039 & .095 \\
\hline $2 a_{5 / 2}$ & $\operatorname{lf}_{7 / 2}$ & 9.5 & .017 & .008 & .051 & & & \\
\hline $2 d_{5 / 2}$ & $I f_{5 / 2}$ & 6.5 & .089 & -.003 & .195 & .100 & .044 & .124 \\
\hline $2 d_{3 / 2}$ & $\mathrm{If}_{7 / 2}$ & 12.0 & .005 & -.016 & .070 & -.065 & -.018 & .064 \\
\hline$l g_{7 / 2}$ & $2 p_{3 / 2}$ & 8.0 & .008 & -.047 & .275 & -.118 & -.052 & .174 \\
\hline$I_{13 / 2}$ & $\operatorname{lf}_{7 / 2}$ & 19.0 & -.023 & -.003 & .085 & -.036 & -.020 & .059 \\
\hline $\operatorname{li}_{13 / 2}$ & $2 p_{3 / 2}$ & 17.0 & -.034 & 0 & .115 & -.042 & -.030 & .098 \\
\hline \multirow[t]{2}{*}{$* 1 h_{11 / 2}$} & $\lg _{9 / 2}$ & 6.5 & & & & .130 & .063 & .216 \\
\hline & & & & & $\mathrm{T}=1.020$ & & & $\mathrm{~T}=.907$ \\
\hline
\end{tabular}


repulsive character of the "spin-flip" component of the $p-p$ force. The "spin-flip" component of the $p-n$ force is weak and attractive which explains the sign and size of $\mathrm{F}_{\mathrm{n}}^{314}(\mathrm{C})$. These conclusions are based on the discussion of Section 3 of Appendix D. The contribution to the transition from the triad (314) is reduced as a result of core polarization. This is a well known result first used to explain the slow M4 $\gamma$-decay of the $Q=-.908 \mathrm{MeV}$ level to the ground state.98,99 The results for $\mathrm{F}_{\mathrm{p}}^{505}(\mathrm{C})$ and $\mathrm{F}_{\mathrm{n}}^{505}(\mathrm{C})$ are similar to those obtained for the core transition densities describing the $\mathrm{L}=2-8$ and $\mathrm{L}=2-6$ transitions in $\mathrm{Zr}^{90}$ and $\mathrm{Ti}^{50}$, respectively . $\mathrm{F}_{\mathrm{p}}^{505}(\mathrm{C}), \mathrm{F}_{\mathrm{n}}^{505}(\mathrm{C})$, and their major components are similar in shape and have the same sign as $\mathrm{F}_{\mathrm{p}}^{505}(\mathrm{D}) . \mathrm{F}_{\mathrm{n}}^{505}(\mathrm{C})$ is larger than $\mathrm{F}_{\mathrm{p}}^{505}(\mathrm{C})$, but $\mathrm{N} / \mathrm{P}=3.84$ is considerably smaller than the values obtained for $\mathrm{Zr}^{90}$ and $\mathrm{Ti}^{50}$ core transition densities. The reason for this is the decreased importance of excitations involving the excess core neutrons. About $66 \%$ of $\mathrm{F}_{\mathrm{p}}^{505}(\mathrm{C})$ and about $46 \%$ of $\mathrm{F}_{\mathrm{n}}^{505}(\mathrm{C})$ is due to particlehole pairs which satisfy the coupling conditions given before. Some fractionization occurs because the overlap of $u_{21}(r) u_{14}(r)$ with $u_{n_{p} p_{p}}(r) u_{n_{h} l_{h}}(r)$ is somewhat more ambiguous than in the case of $\mathrm{Zr}^{90}$ and $\mathrm{Ti}^{50}$. Essentially the same results would be obtained for all the core transition densities if an average energy denominator of $11.1 \mathrm{MeV}$ is used without exception. This is slightly greater than 1 h $\omega$, the value appropriate for negative parity transitions. 
Angular Distributions $\left(\mathrm{Zr}^{90}, \mathrm{Ti}^{50}\right.$, and $\left.\mathrm{Y}^{89}\right)$

Figure 3 shows the angular distributions which have been calculated for the $\mathrm{L}=2-8$ transitions in $\mathrm{Zr}^{90}$ in the $\left(p, p^{\prime}\right)$ reaction at $18.8 \mathrm{MeV}$. The data shown is from Ref. 8 . The results for the $\mathrm{L}=2$ and 4 transitions in $\mathrm{Ti}^{50}$ for the $\left(p, p^{\prime}\right)$ reaction at 17.5 and $40.0 \mathrm{MeV}$ are compared with experiment in Figure 4. The $17.5 \mathrm{MeV}$ data was taken from the literature $\mathrm{I}^{100}$ and the $40.0 \mathrm{MeV}$ data is the unpublished work of B. Preedom. Theoretical differential cross sections obtained for the excitation of the $Q=-.908 \mathrm{MeV}$ level of $Y^{89}$ for incident protons of $18.9 \mathrm{MeV}, 24.5 \mathrm{MeV}$, and $61.2 \mathrm{MeV}$ are compared with experiment in Figure 5. The data comes from Ref. 10, Pef. 101, and Ref. 102, respectively.

In Figure 3 and 4 the solid curves are the results of the completely microscopic calculations and the dashed curves are the results of the calculations which use the macroscopic vibrational model to describe the core with the core parameters fixed from the bound state calculations. The solid curves in Figure 5 are the complete differential cross sections and the dashed curve represents the $L=5$ component of this cross section. The $\mathrm{L}=3$ component is shown only for the $61.2 \mathrm{MeV}$ case where it appears as a center line. The optical parameters used in these calculations are given and referenced in Table 6. The notation is the same as used in Eq. (B.13) and the same geometry is used for the volume and surface imaginary terms. 


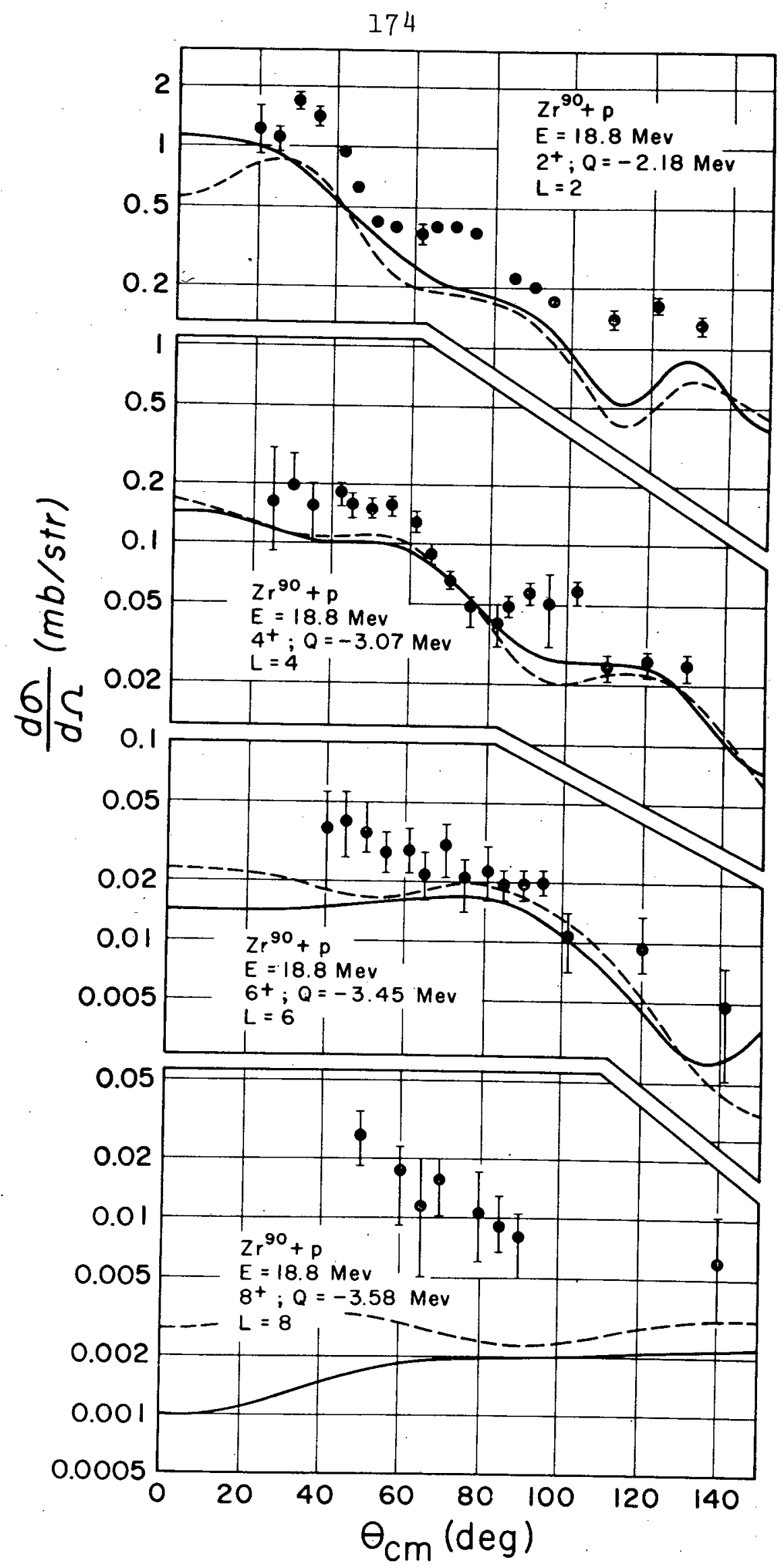

Figure 3.--Differential cross sections for $L=2-8$. transitions in $\mathrm{Zr} 90+\mathrm{p}$ at $18.8 \mathrm{MeV}$. Dashed curves are results obtained using macroscopic vibrational description of core and solid curves are results of completely microscopic calculations. 


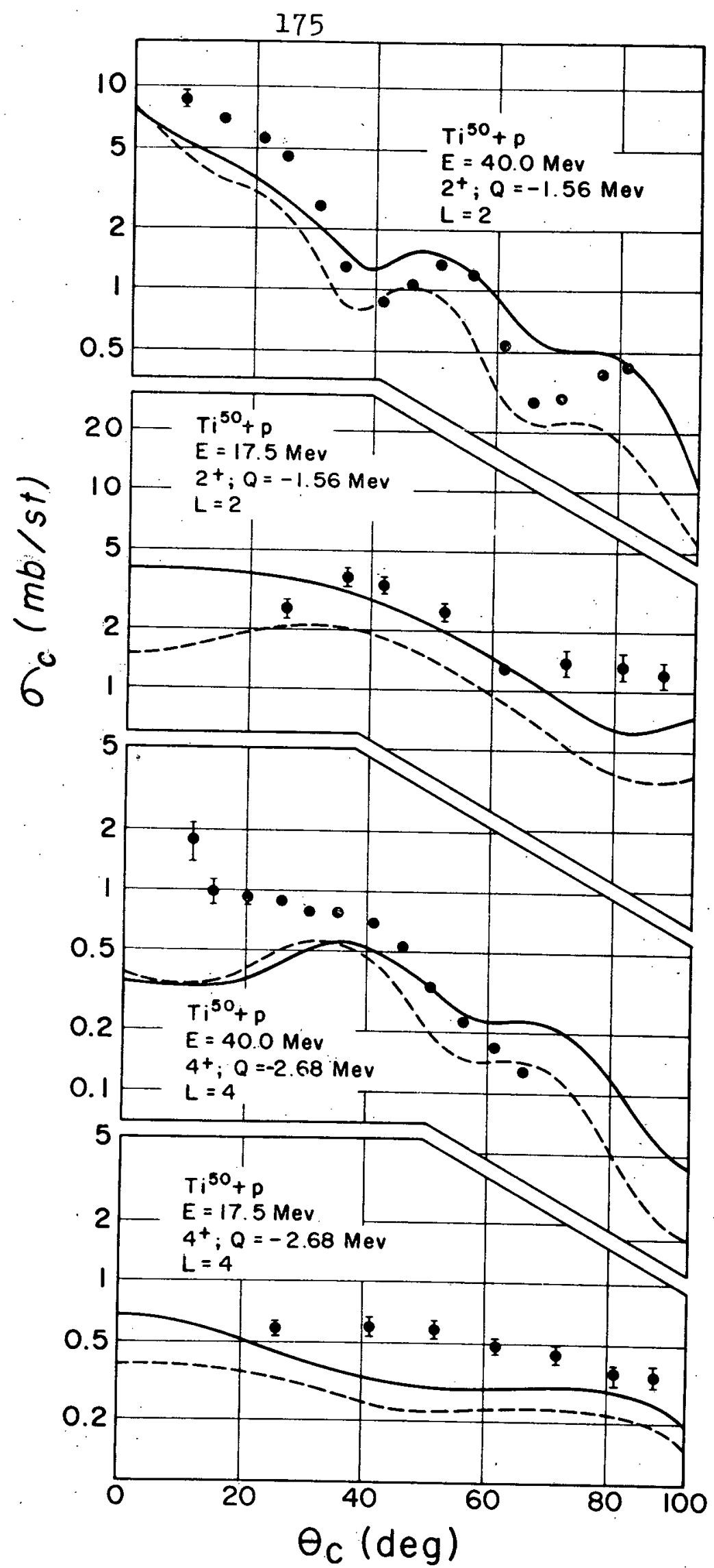

Figure 4.--Differential cross sections for $\mathrm{L}=2$ and 4 transitions in Ti50 $+\mathrm{p}$ at 17.5 and $40 \mathrm{MeV}$. Dashed and solid curves are used the same as in Figure 3. 


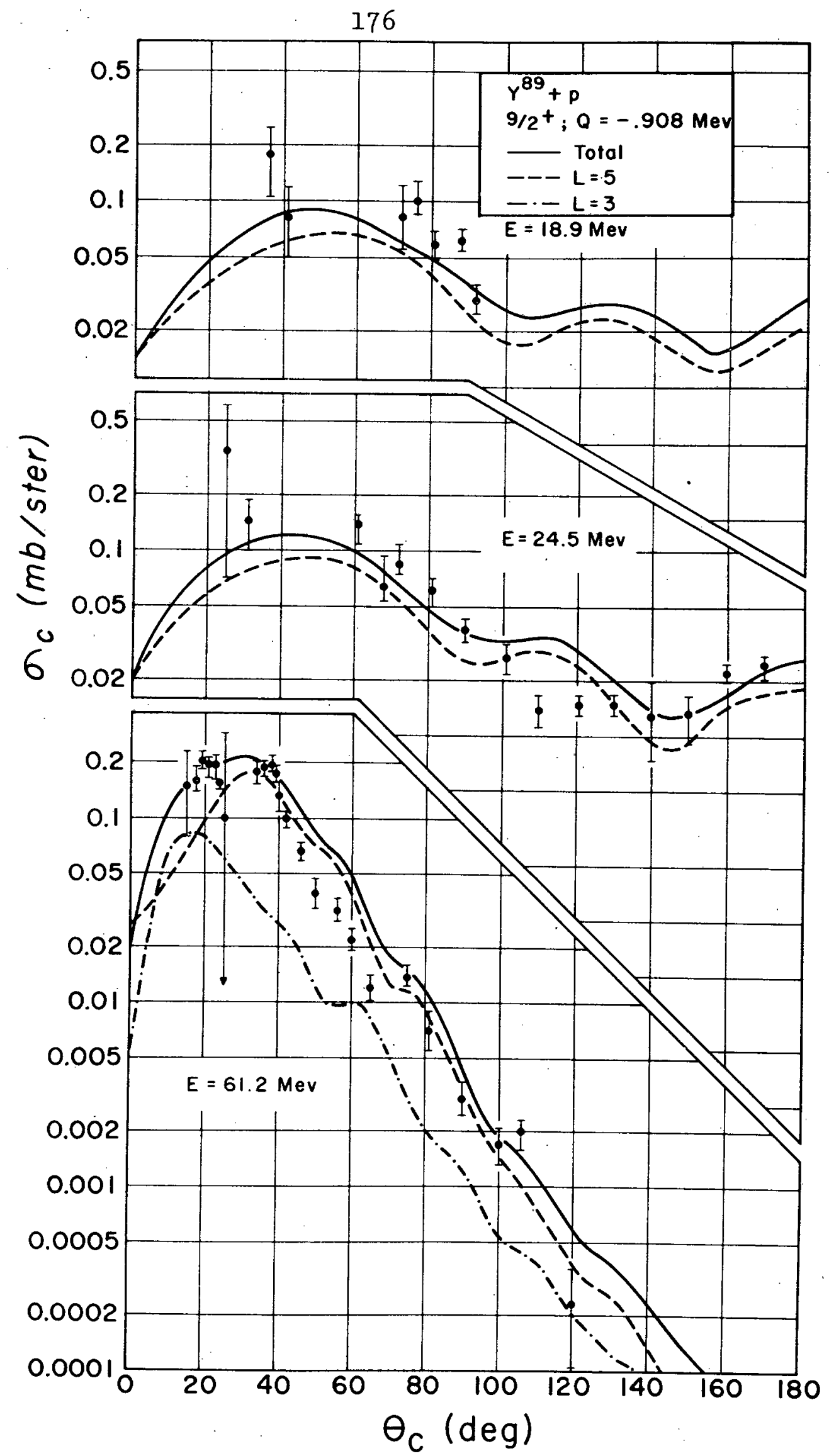

Figure 5.--Differential gross section for excitation of $9 / 2^{+}$ $(Q=-.908 \mathrm{MeV})$ level in $\mathrm{Y}^{89}+\mathrm{p}$ at $18.9,24.5$ and $61.2 \mathrm{MeV}$. Compolete cross sections and $L=5$ component are shown in all cases and the $\mathrm{L}=3$ component is displayed for the $61.2 \mathrm{MeV}$. case. 
TABLE 6.--Optical parameters used in calculating the $2 r^{90}$, Ti 50 , and $\mathrm{Y}^{89}$ angular distributions.
Well depths are in MeV and radii and diffuseness pirameters are in F.

\begin{tabular}{|c|c|c|c|c|c|c|c|c|c|c|c|c|c|}
\hline Target & $\mathrm{E}_{\mathrm{LAB}}(\mathrm{MeV})$ & $\mathrm{V}$ & W & $\mathrm{W}_{\mathrm{D}}$ & $r_{0}$ & a & $r_{0}^{\prime}$ & $a^{-}$ & $\mathrm{V}_{\mathrm{s}}$ & ${ }^{r_{\text {so }}}$ & $a_{\text {so }}$ & $r_{c}$ & $\operatorname{Re} f^{\prime}$. \\
\hline $2 r^{90}$ & 18.8 & 52.0 & 0.0 & 9.25 & 1.20 & .70 & 1.25 & .65 & 6.2 & 1.20 & .70 & 1.25 & 8 \\
\hline $\mathrm{T} 150(1)$ & 17.5 & 48.0 & 0.0 & 11.0 & 1.25 & .65 & 1.25 & .47 & 0.0 & - & - & 1.25 & 100 \\
\hline $\mathrm{T} 1^{50(1)}$ & 40.0 & 44.8 & 8.1 & 0.0 & 1.169 & .755 & 1.403 & .441 & 6.51 & 1.169 & .755 & 1.20 & $76^{a}$ \\
\hline$y^{89}$ & 18.9 & 52.6 & 0.0 & 9.8 & 1.20 & .70 & 1.25 & .65 & 5.70 & 1.20 & .70 & 1.25 & 10 \\
\hline$Y^{89}$ & 24.5 & & . & SAME AS & $18.9 \mathrm{Me}$ & & & & & $\cdot$ & & & \\
\hline$y^{89}$ & 61.2 & 39.5 & 5.12 & 2.54 & 1.20 & .69 & 1.40 & .53 & 6.00 & 1.20 & .69 & $1.20^{\circ}$ & 102 \\
\hline $\mathrm{T}^{50(2)}$ & $17 \cdot 5$ & 48.3 & 0.0 & 10.68 & 1.236 & .60 & 1.261 & .52 & 10.0 & 1.236 & .60 & 1.236 & 103 \\
\hline $\mathrm{TI}^{50(2)}$ & 40.0 & 44.85 & 7.82 & 1.14 & 1.16 & .75 & 1.37 & .630 & 6.04 & 1.064 & .738 & 1.25 & $104^{b}$ \\
\hline
\end{tabular}


Two sets of parameters are given for $\mathrm{Ti}^{50}$. The first set (1) was used for the completely microscopic calculations and the second set (2) were used in the calculations using the macroscopic vibrational description of the core. Set (2) give better fits to the elastic scattering data, but were not available until the microscopic calculations were completed. As the differences between the two sets of parameters are not sufficiently large to alter the conclusions of this work, the microscopic calculations were not repeated with the improved parameters.

The overall agreement of the theoretical angular distributions with experiment is fairly good with the possible exception of the $L=8$ transition in $\mathrm{Zr}^{90}$. The general tendency is for the theoretical results to underestimate the data slightly (by factors less than two), but it appears as if at least a rough account of the relative magnitude of the differential cross sections of different multipolarity in $\mathrm{zr}^{90}$ and $\mathrm{Ti}^{50}$ has been achieved. The results of the microscopic calculations are in good agreement with the results of the calculations which use the microscopic vibrational description of the core. ${ }^{\dagger}$ This is expected as the latter are only intended to display, more directly, the relation between the renormalization of the force acting between bound nucleons and the

The agreement between the microscopic and macroscopic results for $\mathrm{Ti} 50$ is a little poorer than for Zr 90 . This is attributed mostly to the differences in the energy denominators used in this work and in Ref. 25 which were
previously pointed out. 
renormalization of the force between a bound and an unbound nucleon. The macroscopic model gives angular distributions with somewhat better shapes than the microscopic calculations. This is particularly evident for the $L=2$ transitions. It is interesting to note that the prescription for calculating cross sections which is being followed here leads to the conclusion that the $L=3$, abnormal parity component of the $Y^{89}$ cross section is appreciable. Other analyses have assumed that the angular distribution is totaliy due to $L=5$ transfer. 10,101,102 The presence of the $L=3$ component of the cross section is supported by the data--particularly at 61.2 MeV where it broadens the forward peak in the angular distribution. The apparent dip in this angular distribution at about $25^{\circ}$ is not reproduced by the calculation. In order to reproduce this feature of the data both the relative magnitudes, widths, and peak positions of the $\mathrm{L}=3$ and $\mathrm{L}=5 \mathrm{com}-$ ponents of the angular distribution would have to be given precisely. The approximations and assumptions employed in this work are too crude to give such fine details of angular distributions.

For the sake of completeness the integrated cross sections corresponding to the microscopic results of Fig. 3, 4 , and 5 are decomposed into their direct and exchange components in Table 7 . The results in the table are consistent with the observations of Chapter 5. 
TABLE 7.--Decomposition of integrated cross sections corresponding to results shown in Fig. 3, 4, and 5 into direct and exchange cumponents. All cross sections are'given in mb.

\begin{tabular}{|c|c|c|c|c|c|c|}
\hline Target & $E_{L A B}(\mathrm{MeV})$ & $\mathrm{L}$ & $\sigma_{\text {dir }}$ & $\sigma_{e x}$ & $\sigma_{\mathrm{T}}$ & $\sigma_{e x} / \sigma_{d i r}$ \\
\hline \multirow{4}{*}{$\mathrm{Zr}^{90}$} & \multirow{4}{*}{18.8} & 2 & .997 & .482 & 2.84 & .481 \\
\hline & & 4 & .165 & .156 & .627 & .945 \\
\hline & & 6 & .0207 & .0550 & .137 & 2.66 \\
\hline & & 8 & .00116 & .0163 & .0236 & 14.1 \\
\hline \multirow{4}{*}{$\mathrm{Ti}{ }^{50}$} & \multirow[t]{2}{*}{17.5} & 2 & 4.89 & 2.70 & 14.5 & .553 \\
\hline & & 4 & .643 & .911 & 2.94 & i. 42 \\
\hline & \multirow[t]{2}{*}{40.0} & 2 & 3.93 & .884 & 8.30 & .224 \\
\hline & & 4 & .680 & .340 & 1.86 & .500 \\
\hline \multirow{6}{*}{$y^{89}$} & \multirow[t]{2}{*}{18.9} & 3 & .0402 & .0303 & .127 & .754 \\
\hline & & 5 & .0988 & .143 & .441 & 1.45 \\
\hline & \multirow[t]{2}{*}{24.5} & 3 & .0496 & .0329 & .150 & .664 \\
\hline & & 5 & .124 & .165 & .532 & 1.33 \\
\hline & \multirow[t]{2}{*}{61.2} & 3 & .0768 & .00473 & .116 & .0616 \\
\hline & & $\cdot 5$ & .189 & .0330 & .361 & .175 \\
\hline
\end{tabular}

Form Factors for $\mathrm{L}=2$ Transitions in $\mathrm{Zr}^{90}$ and $\mathrm{Ti}^{50}$

The form factors for the microscopic calculations are obtained by folding in the appropriate multipole coefficient of the $\mathrm{K}-\mathrm{K}$ force and exchange interaction with the complete transition densities obtained by combining the valence and core transition densities. The folding procedure is defined in Eq. (2.59"). When the macroscopic vibrational. 
description of the core is used the form factors are defined by Eq. (D.13) where $n_{F} L S J(r)$ denotes the valence form factor.

The form factors given by the microscopic and macroscopic models for the $L=2$ transition in $\mathrm{Zr}^{90}$ at $18.8 \mathrm{MeV}$ and in $\mathrm{Ti}^{50}$ at 17.5 and $40 \mathrm{MeV}$ are compared in Fig. 6, i, and 8 , respectively. The valence form factors are labeled $D$ and shown as a solid line in the figures. They are the same in both the microscopic and macroscopic pictures. The total form factors, which include the effect of core polarization, as given by the macroscopic model are represented by dashed curves Iabeled $D+C$ (Macro). These are complex and both the real and imaginary components are shown in the figures. The total microscopic form factors are represented by center lines labeled $D+C$ (Micro). These are real. Strictly speaking one expects the projectile-target interaction to be complex which would lead to complex form factors in the microscopic calculations also.

The total microscopic form factors and the real part of the total macroscopic form factors are similar in shape to the direct form factors, although they both peak at siight Iy larger radii. The total macroscopic form factors peak at the largest radii in all cases shown. The better angular distributions given by the macroscopic calculations is attributable largely to the latter observation although the imaginary part of the macroscopic form factors does play some part in the improved shapes. 


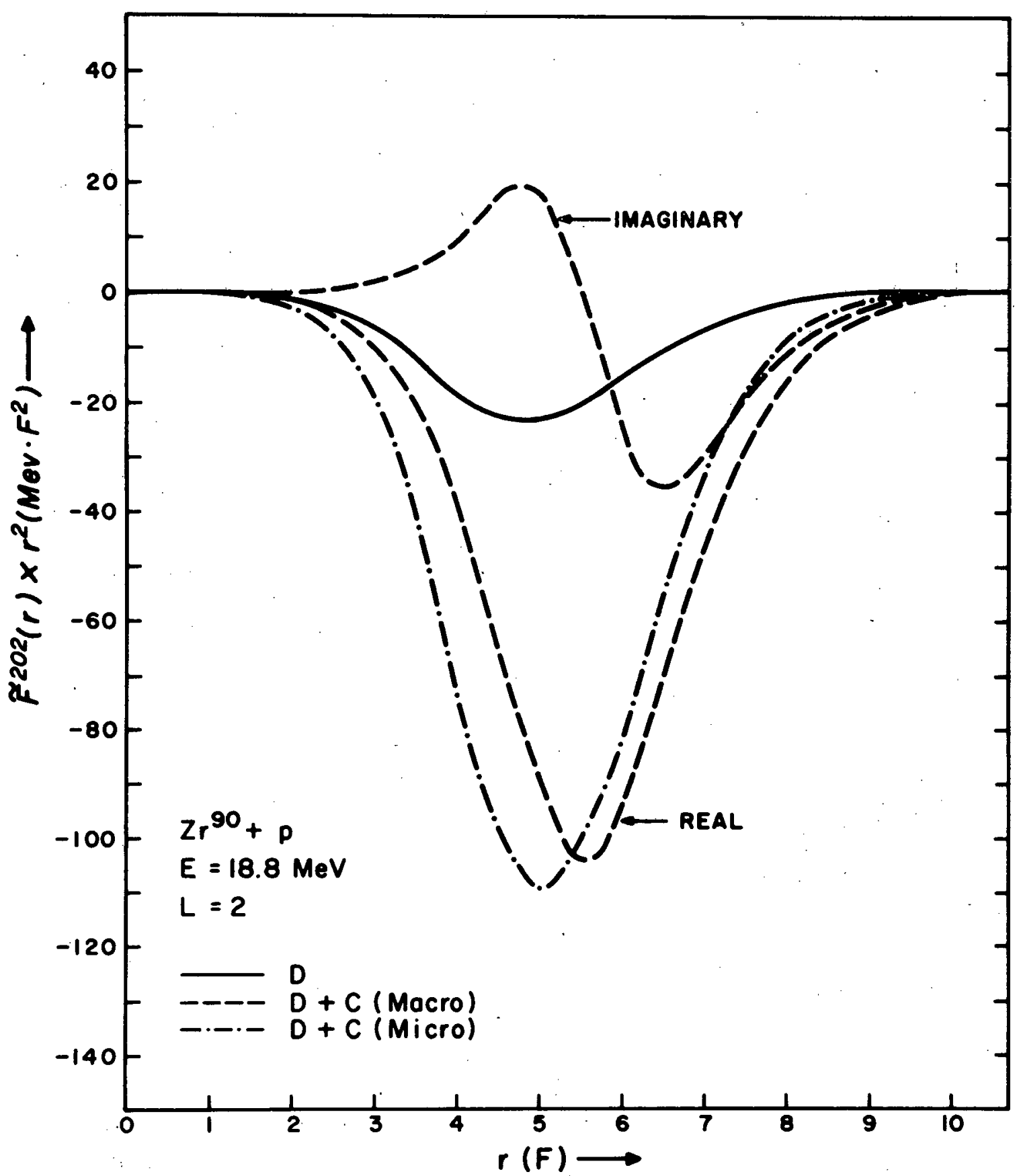

Figure 6.--Direct form factor and total form factors obtained in the macroscopic and microscopic calculations for the $L=2$ transition in $\mathrm{Zr}^{90}+\mathrm{p}$ at $18.8 \mathrm{MeV}$. 


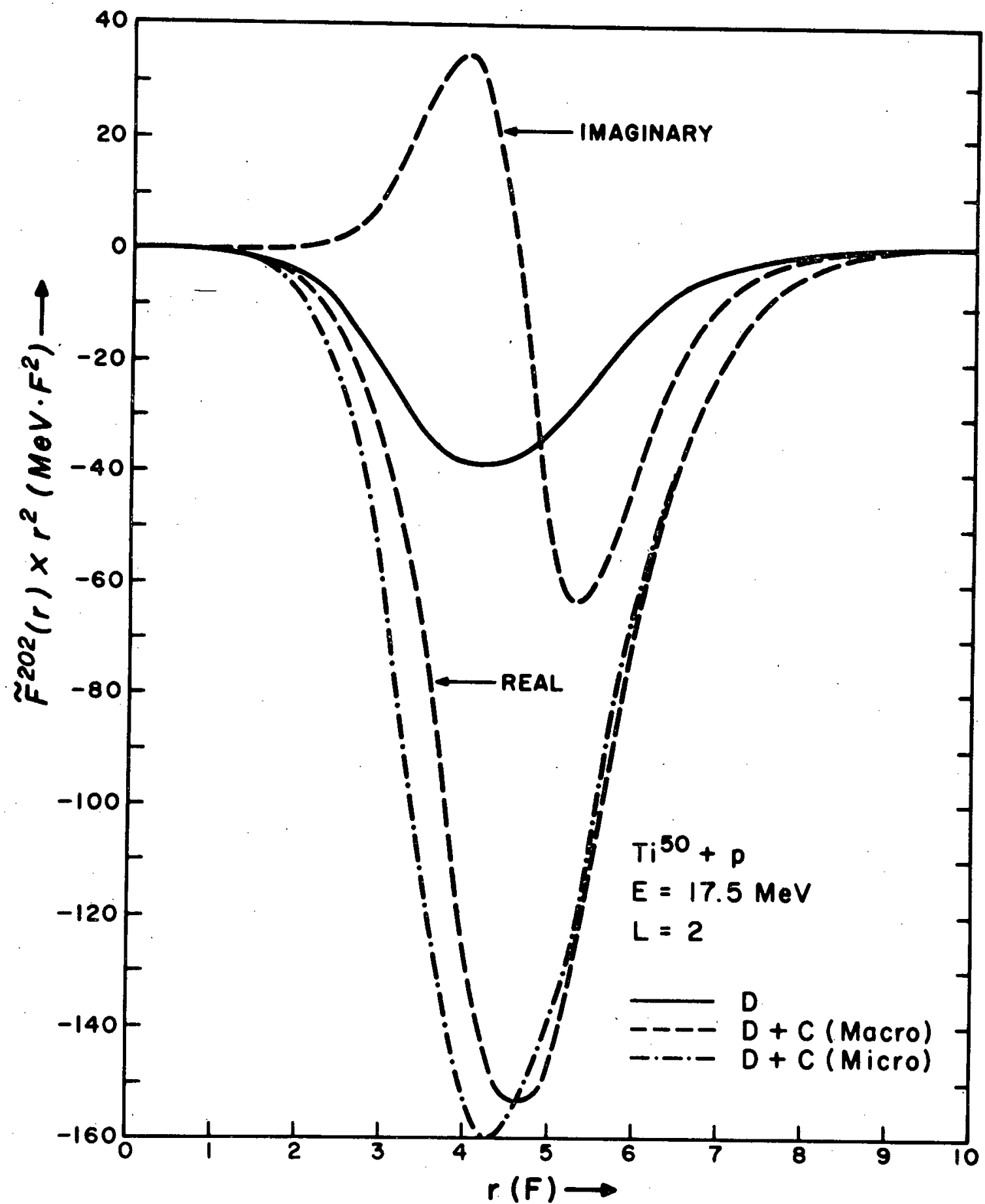

Figure 7:- Same as Figure 6 for $L=2$ transition in $\mathrm{Ti}^{50}+\mathrm{p}$ at $17.5 \mathrm{MeV}$. 


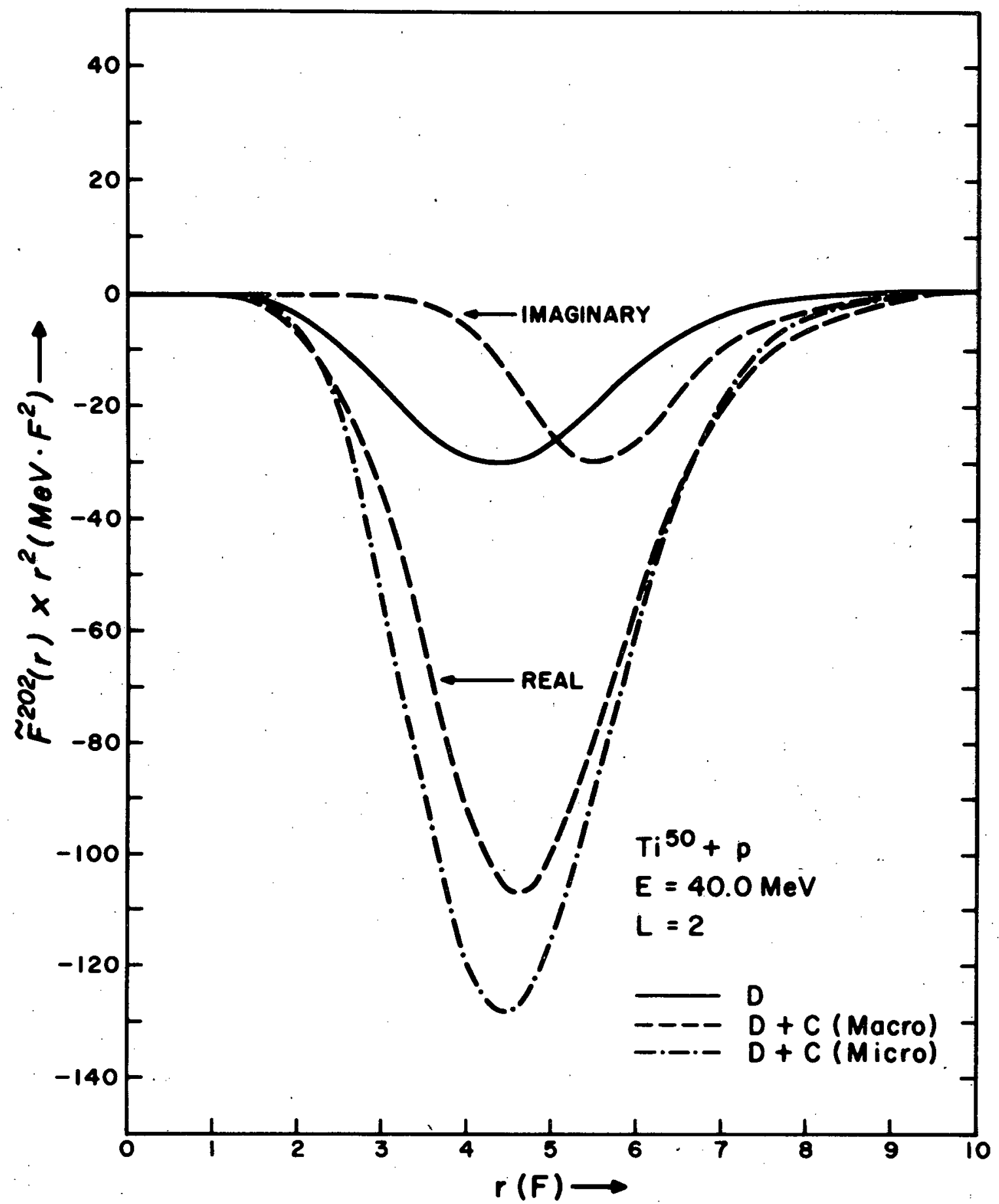

Figure 8.--Same as Figure 7 for $\mathrm{Ti} 50+\mathrm{p}$ at $40 \mathrm{MeV}$. 


\section{Enhancement Factors}

In order to examine more carefully the role of core polarization in these results, the square of the enhancement factors obtained in these calculations are given in Table 8. These are simply the ratio of the integrated cross section obtained with core polarization to the integrated cross section obtained without core polarization. They are denoted by $\varepsilon_{p}^{2}$ where the subscript $p$ appears because the valence nucleons are protons in all cases being considered. Except for the abnormal parity $L=3$ component of the $Y^{89}$ cross section, the values of $\varepsilon_{p}^{2}$ are of the order of 10. This illustrates that core polarization plays an extremely important role in the $\left(p, p^{\prime}\right)$ reaction. Experimental values of $\varepsilon_{\mathrm{p}}^{2}$ are given for the transitions in $\mathrm{Zr}^{90}$ and $\mathrm{Ti}^{50}$. For the $L=2$ and $L=4$ transitions in $2 r^{90}$ these have been obtained by normalizing the theoretical angular distribution for the valence transition to the data at $40^{\circ}$. For the $L=6$ and $L=8$ transitions in $\mathrm{Zr}^{90}, 60^{\circ}$ and $70^{\circ}$ were used to compute $\varepsilon_{\mathrm{p}}^{2}$. For $\mathrm{Ti}^{50}$ at $17.5 \mathrm{MeV}, \varepsilon_{\mathrm{p}}^{2}$ was determined by comparing the theory and data at $40^{\circ}$, but at $40 \mathrm{MeV}$ the hump at $51^{\circ}$ in the experimental $\mathrm{L}=2$ angular distribution and the flat spot at $35^{\circ}$ in experimental $\mathrm{L}=4$ angular distribution were used for the point of normalization. In all cases good "eye" fits to the data have been achieved. Experimental enhancement factors have not been obtained for the $\mathrm{Y}^{89}$ transition because the cross section contains two components. 
TABLE 8.--Theoretical and experimental values for the square of the enhancement factors corresponding to the results of Fig. 3, 4, and 5. For prescription used to calculate $\varepsilon_{\mathrm{p}}^{2}(\operatorname{Exp})$

\begin{tabular}{|c|c|c|c|c|c|}
\hline Target & $\mathrm{E}_{\mathrm{LAB}}(\mathrm{MeV})$ & $\mathrm{L}$ & $\varepsilon_{p}^{2}($ Micro $)$ & $\varepsilon_{p}^{2}($ Macro $)$ & $\varepsilon_{p}^{2}(\operatorname{Exp})$ \\
\hline \multirow{4}{*}{$\mathrm{Zr}^{90}$} & \multirow{4}{*}{18.8} & 2 & 18.9 & 16.5 & 33.2 \\
\hline & & 4 & 12.7 & 12.2 & 20.3 \\
\hline & & 6 & 9.56 & 11.0 & 18.4 \\
\hline & & 8 & 7.62 & 11.0 & 55.2 \\
\hline \multirow[t]{2}{*}{$\mathrm{Ti}^{50}$} & \multirow[t]{2}{*}{$17 \cdot 5$} & 2 & 17.2 & 10.8 & 18.9 \\
\hline & & 4. & 12.8 & 9.1 & 19.4 \\
\hline \multirow[t]{2}{*}{$\mathrm{Ti}^{50}$} & \multirow[t]{2}{*}{40.0} & 2 & 19.8 & $13 \cdot 7$ & 19.8 \\
\hline & & 4 & 14.9 & 12.7 & 18.2 \\
\hline \multirow[t]{2}{*}{$y^{89}$} & \multirow[t]{2}{*}{18.9} & 3 & .629 & - & - \\
\hline & & 5 & 9.14 & - & - \\
\hline \multirow[t]{2}{*}{$y^{89}$} & \multirow[t]{2}{*}{24.5} & 3 & .641 & - & - \\
\hline & & 5 & 9.55 & - & $=$ \\
\hline \multirow[t]{2}{*}{$y^{89}$} & \multirow[t]{2}{*}{61.2} & 3 & .617 & - & - \\
\hline & & 5 & 10.3 & - & - \\
\hline
\end{tabular}


Beyond any uncertainties associated with normalizing the theoretical results to the experimental data, the experimental values of $\varepsilon_{p}^{2}$ given in Table 8 are subject to any errors contained in the approximate treatment of antisymmetrization used in this work. For example consider the results of Love et al ${ }^{74}$ which were discussed in the note added to Chapter 5. Using the central part of H-J force for the projectile-target interaction and treating antisymmetrization exactly, for the $L=2$ valence transition in $2 r^{90}$ they obtain $\sigma_{\mathrm{dir}}=.0412 \mathrm{mb}, \sigma_{\mathrm{ex}}=.00415 \mathrm{mb}$, and $\sigma_{\mathrm{T}}=.0689 \mathrm{mb}$ with $\sigma_{\mathrm{ex}} / \sigma_{\mathrm{dir}}=.1$. The results of this work are $\sigma_{\text {dix }}=.0524 \mathrm{mb}, \sigma_{\text {ex }}=.0246 \mathrm{mb}$, and $\sigma_{\mathrm{T}}=.150 \mathrm{mb}$ with $\sigma_{\text {ex }} / \sigma_{\mathrm{dir}}=.470$. The first set of results gives $\varepsilon_{\rho}^{2}(\operatorname{Exp})=72.5$ for the $\mathrm{L}=2$ transition in $\mathrm{zr}^{90}$.

Taking for $\sigma_{\text {dir }}$ the values obtained in this work for the $K-K$ force, using the $\sigma_{\text {ex }} / \sigma_{\text {dir }}$ ratios of Love et al shown in Fig. (5.1'), and assuming maximum interference (see Eq. (5.3)) suggests that a proper treatment of exchange might lead to the following modifications of the $z^{90}$ resurts which have been shown.

(1) Values of $\varepsilon_{p}^{2}(\operatorname{Exp})=54.7,33.8,23.2$, and 35.1 might result for $\mathrm{L}=2,4,6$, and 8 .

(2) The microscopic angular distributions for the $\mathrm{L}=2,4$, and 6 transitions of Fig. 3 may be reduced by factors of $1.65,1.66$, and 1.26 , respectively, while the $L=8$ angular distribution may be increased by 1.57 . Here it has been assumed that the complete 
differential cross sections will be effected in the same way as the differential cross section for the valence transition. This is not strictly true since neutron excitations contribute to the former and the $n-p$ and $p-p$ forces do not have the same radial shape.

(3) The macroscopic angular distributed of Fig. 3 may be multiplied by factors of $1 / 1.12,1 / 1.14,1 / 1.07$, and 1.15 in the order $\mathrm{L}=2-8$. These cross sections are more stable than the microscopic ones since the core contributions are not effected by the uncertainties in question.

(4) Under the assumption of (2) the values of $\varepsilon_{p}^{2}$ (Micro) will not be changed.

(5) From (3) it follows that $\varepsilon_{p}^{2}$ (Macro) will be 24.2 , $17.8,13.9$, and 8.09 for $\mathrm{L}=2-8$.

The main point here is that the results of this work might be biased so as to improve the agreement of theory and experiment for $L=2-6$ transfers.

The indicated modifications improve the consistency of theory and experiment for $L=2-8$, but at the same time result in somewhat poorer absolute agreement. With the modifications the microscopic $L=2$ cross section is too low by a factor of 2.9 while the $L=8$ cross section is a factor of 4.6 under the data. Inclusion of the $L=7$ non-normal transfer in the $8^{+}$calculation might then remove most of the discrepancy between the two results. Finally observe that the agreement 
between the microscopic and macroscopic results is not strongly effected by the uncertainties due to the exchange approximation, although the microscopic results for the $L=2,4$, and 6 transitions will be shifted downward $20 \%$ with respect to the macroscopic results while the $L=8$ results might be brought into essentially complete agreement. The fact that the macroscopic cross sections may be larger than the microscopic cross sections could reflect that a larger value of $\left\langle k_{v}\right\rangle$ should be used in these calculations.

The value of $\varepsilon_{p}^{2}(\operatorname{Exp})$ for the $L=2$ and $L=4$ transitions in $\mathrm{Ti}^{50}$ are found to be about equal, roughly 19 , and the data provides no indication that this number varies with energy. It would be useful to have results with exchange treated exactly to check these points. Except for the magnitude of $\varepsilon_{p}^{2}$ it is expected that the observations will be upheld. Guessing that the cross sections for these valence transitions are being overestimated by the same amount as for $\mathrm{Zr}^{90}$ leads to a modified value for $\varepsilon_{\mathrm{p}}^{2}$ (Exp) of about 31 at $17.5 \mathrm{MeV}$.

It is found that $\varepsilon_{p}^{2}$ (Macro) for the $L=2$ transition is a little larger than for the $\mathrm{L}=4$ transition and both are too small by about a factor of two at $17.5 \mathrm{MeV}$. They also increase a little with energy. $\varepsilon_{p}^{2}$ (Macro) for the $L=2$ transition at $17.5 \mathrm{MeV}$ might be modified to a value of 13 which is about 2.3 times smaller than the modified experimental value. The fact that $\varepsilon_{p}^{2}$ (Micro) are larger than $\varepsilon_{p}^{2}$ (Macro) 
has already been explained. The values for $\varepsilon_{p}^{2}$ (Micro) increase slightly with energy as is expected since the shorter range $n-p$ force is a factor in the complete cross section while only the $p-p$ force is involved in the valence transition. Calculations have been carried by Satchler et al. 67 for the single particle transition in $Y^{89}$ at 18.9 and $61.4 \mathrm{Me}$. The H-J force has been used and exchange has been treated exactly. Comparing their results with the results of this work indicates that the approximate treatment of exchange is not introducing any serious discrepancies here. This is expected as the dominant multipole is $L=5$ in this case. The comparison also indicates that somewhat smaller (less than a factor of 2) cross sections would be obtained with the H-J force. This is also true for the $\mathrm{L}=2$ transition in $\mathrm{Zr}^{90}$ where the $K-K$ force gives the modified experimental value, $\varepsilon_{p}^{2}=54.7$, while $\varepsilon_{p}^{2}=72$ is obtained for the $H-J$ force from Ref. 74 . In any event it appears as if the results obtained here for this transition in $Y^{89}$ are somewhat better than those obtained for $\mathrm{Ti}^{50}$ and $\mathrm{Zr}^{90}$.

$\underline{L}=0$ Transition in $\mathrm{Zr}^{90}$

An experimental differential cross section is available for the excitation of the $0^{+}(Q=-1.75 \mathrm{MeV})$ level of $\mathrm{Zr}^{90}$ in the $\left(p, p^{\prime}\right)$ reaction at $12.7 \mathrm{MeV} .{ }^{105}$ Ref. 8 also gives an upper limit for this cross section for incident protons of 
$18.8 \mathrm{MeV}$. This is about $20 \mu \mathrm{b}$ between $40^{\circ}$ and $60^{\circ}$. A calculation of the differential cross section for the valence transition at $18.8 \mathrm{MeV}$ gives a result which is in agreement with this upper limit. The decomposition of the integrated cross section for this case is $\sigma_{\mathrm{dir}}=.0453 \mathrm{mb}, \sigma_{\mathrm{ex}}=.0392 \mathrm{mb}, \sigma_{\mathrm{T}}=.169 \mathrm{mb}$, and $\sigma_{e x} / \sigma_{d i r}=.865$. This ratio is much larger than $\sigma_{e x} / \sigma_{d i r}=.22$ which is obtained. when it is assumed that only $\lg _{9 / 2}$ protons are involved in the $L=0$ transition (see Fig. 5.1'). This same effect was observed for Yukawa forces in the discussion of Fig. 5.1. Core polarization gives $\varepsilon_{p}^{2}=9.35$ for this transition which destroys the agreement with experiment. Assuming that $\sigma_{\text {ex }} / \sigma_{\text {dir }}$ is 10 times too large which is inferred from Fig. 5.1' leads to a result which is only about 4 times greater than the upper limit.

A calculation with core polarization was made for comparison with the $12.7 \mathrm{MeV}$ data. Optical parameters were taken from Ref. 105. The direct and total (direct plus exchange) differential cross sections are shown with the data in Fig. 9. The shape of the theoretical cross sections are not in good agreement with the data and it is seen that there is a large exchange contribution. Again assuming that the effect of exchange is being overestimated leads to a result which is not very different than the direct differential cross section shown. This is in accord with the data insofar as overall magnitude is concerned. Love et $\underline{a l}^{74}$ have indicated a value of 10 is needed for $\varepsilon_{p}^{2}$ based on their calculation of the differential cross section for the valence transition using 


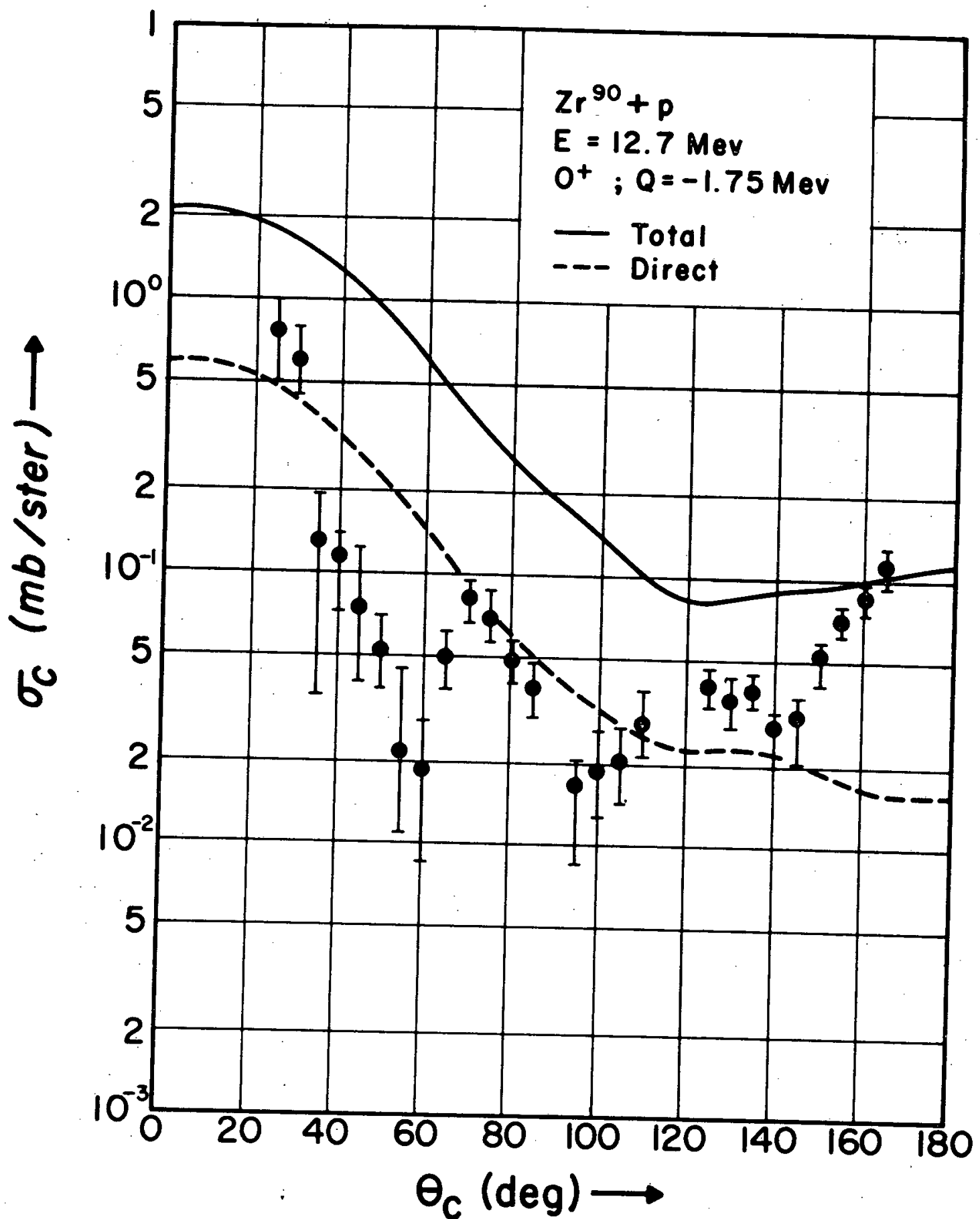

Figure 9.--Differential crose sections for $\mathrm{L}=0$ transition in $\mathrm{Zr90}+\mathrm{p}$ at $12.7 \cdot \mathrm{MeV}$. Direct and total (direct + exchange) cross sections are shown. Core polarization is included. 
the $K-B$ reaction matrix. This is roughly the value obtalned in this work.

Note that the data has a deep minimum at $60^{\circ}$ - the region where the upper limit of the $18.8 \mathrm{MeV}$ cross section was fixed - and observe that because of the poor shape agreement this point is badly overestimated. It has been suggested that the shape of the theoretical result can be improved by damping the form factor in the nuclear interior. ${ }^{9,105}$ An angular distribution with a better shape has been obtained in Ref. 105 from a macroscopic form factor representing a breathing mode. 65

\section{Effective Charges}

'Table 9 contains the effective charges for the electric $2^{\mathrm{L}}$-pole component of the transition rates for the transitions under consideration. Experimental values are given for the $L=2$ transitions. These have been extracted from transition rates given in the indicated references on the basis of the harmonic oscillator wave functions used in this work. Note that there are two experimental values given for the quadrupole effective charge in $\mathrm{Zr}^{90}$. The two numbers do not agree with each other and the larger number is the most recent result.

The results for e eff(Micro) are simply the square roots of the ratios of the $B(E L)$ computed with the complete proton transition density, $\mathrm{F}_{\mathrm{p}}^{\mathrm{LOL}}(\mathrm{T})$, to the $\mathrm{B}(\mathrm{EL})$ computed with the valence transition density, $F_{p}^{L O L}(D)$. For the 
TABLE 9.--Effective charges for electric $2^{L}$-pole component of transition amplitudes for the transitions under consideration in $\mathrm{Zr} 90$, Ti50, and $\mathrm{Y} 89$.

\begin{tabular}{c|c|cccc}
\hline Nucleus & $L$ & $e_{\text {eff (Micro) }}$ & $\bar{e}_{\text {eff }}$ & $e_{\text {eff }}$ (Macro) & $e_{\text {eff }}$ (Exp) \\
\hline \multirow{2}{*}{$\mathrm{Zr}^{90}$} & 2 & 1.23 & 1.79 & 2.08 & $\begin{array}{l}2.3 \pm .4^{15,106} \\
3.2 \pm .2\end{array}$ \\
& 4 & 1.19 & 1.65 & 1.73 & - \\
& 6 & 1.13 & 1.51 & 1.52 & - \\
\hline $\mathrm{Ti}^{50}$ & 2 & 1.08 & 1.34 & 1.41 & $1.8 \pm .2^{107}$ \\
& 4, & 1.19 & 1.67 & 1.92 & - \\
\hline $\mathrm{Y}^{89}$ & 5 & 1.18 & 1.54 & 1.64 & - \\
\hline
\end{tabular}

definition of $B(E L)$ see Eq. (C.17) or Eq. (D.54). Eq. (D.23) has been used to calculate e eff(Macro). In these calculations it has been assumed that $R_{c}^{L} /<r{ }^{L}>=1$. Actual values of this quantity based on reasonable finite well wave functions for various orbitals in different nuclei vary from .6-1.5.15,16,108 The quantity $\bar{e}_{\text {eff }}$ is obtained by taking $\mathrm{F}_{\mathrm{p}}^{\mathrm{LOL}}(\mathrm{C})$ to be given by $\frac{1}{2}\left[\mathrm{~F}_{\mathrm{p}}^{\mathrm{LOL}}(\mathrm{C})+\mathrm{F}_{\mathrm{n}}^{\mathrm{LOL}}(\mathrm{C})\right]$.

The quadrupole effective charges given by the microscopic model fall far short of the experimental values. The macroscopic model gives reasonable agreement with experiment if the smaller value for the $\mathrm{L}=2$ effective charge in $\mathrm{Zr}^{90}$ is assumed to be correct. The values of $\bar{e}_{\text {eff }}$ are in better agreement with e eff(Macro) and e eff(Exp) than are e eff(Micro). The substitution used in calculating $\bar{e}_{\text {eff }}$ is strictly valid 
only in the limit of iso-scalar core excitation - a condition which might be closer to reality than the microscopic calculations indicate because of the correlations between core nucleons which are neglected in that picture. Note that the values for e eff (Macro) are subject to a assumption similar to the one made in calculating $\bar{e}_{\text {eff }}$, i.e. only the overall effect of core polarization is contained in the values of $\left\langle k_{v}\right\rangle^{2} \theta_{L}$ extracted from the Kuo-Brown matrix elements and an independent assumption as to how this effect is divided up into neutron and proton components is made in writing down Eq. (D.23).

It is concluded that the proton-neutron imbalance predicted by the microscopic calculations is not consistent with experiment. Experiment appears to favor something more like iso-scalar core excitation. This point will be examined in more detail in a short while. It should also be pointed out that the inclusion of those proton-proton hole excitations where the proton is in the valence orbital will not be sufficient to remedy this situation. ${ }^{\dagger}$ Finally, there is no information indicating that these calculations are giving a fair description of the relative variation of eff as a function of multipole. Additional experimental $\gamma$-decay data would prove useful in examining this point.

\section{Coupling to Physical Core States}

Collective model analysis of the first $2^{+}$excitation in $\mathrm{Sr}^{88}$ which has been observed at $1.84 \mathrm{MeV}$ in the $\left(\mathrm{p}, \mathrm{p}^{\prime}\right)$

tsee note at end of this chapter. 
reaction at $18.9 \mathrm{MeV}$ yields the value $\beta_{2}=.13 .^{10}$ Several other low lying $2^{+}$states are also observed. A $4^{+}$state is believed to exist at $4.05 \mathrm{MeV}$ but it has not been resolved experimentally. The first $2^{+}(Q=-3.82 \mathrm{MeV})$ and first $4^{+}(\mathrm{Q}=-6.33 \mathrm{MeV})$ levels in $\mathrm{Ca}^{48}$ have also been observed in the $\left(p, p^{\prime}\right)$ reaction at $25,30,35$, and $40 \mathrm{MeV} .109$ values of $\beta_{2} \sim 17$ and $\beta_{4} \sim 09$ have been extracted from a collective model analysis of this data. From Eq. (B.17) it follows that $\mathrm{C}_{2}=272 \mathrm{MeV}$ for the $\mathrm{Sr}^{88}$ levels and $\mathrm{C}_{2}=330 \mathrm{MeV}$ and $\mathrm{C}_{4}=3516 \mathrm{MeV}$ for the $\mathrm{Ca}^{48}$ levels. From the experimental data it is estimated that first $4^{+}$state in $\mathrm{Sr}^{88}$ has $\beta_{4^{\sim}} .04$ which gives $\mathrm{C}_{4} 10^{4}$. These values of $\mathrm{C}_{2}$ are comparable to those which appear in Table 1 of this chapter. The values of $\mathrm{C}_{4}$ given here are roughly an order of magnitude larger than the corresponding values appearing in that table.

The appearance of phonons in the core nuclei which have strengths comparable to the effective core phonon associated with the uncorrelated particle-hole model introduces serious reservations concerning the use of this model. Kuo has already made this point. 90 A case where such a core phonon is dominant will be discussed in Section 3 of this chapter. The general consistency of $\beta_{2}$ values extracted from analysis of the $\left(p, p^{\prime}\right)$ reaction and $\left(e, e^{\prime}\right)$ experiments indicates that such phonons have comparable proton and neutron transition densities; therefore, they will give a better account of the charge and mass polarization effects in these $L=2$ transitions than the particle-hole model does. 
The large $\mathrm{C}_{4}$ values for the first $4^{+}$states in $\mathrm{Ca}^{48}$ and $\mathrm{Sr}^{88}$ could be indicative that the particle-hole model might be better for the states of higher multipolarity; however, the results obtained for $L=4$ transitions do not compare more favorably with experiment than those for $L=2$ transitions. No strong $5^{-}$state has been observed in $\mathrm{Sr}^{88}$. The results obtained for the single particle transition in $\mathrm{Y}^{89}$ compare quite well with experiment--better than those for $\mathrm{Ti}^{50}$ and $\mathrm{Zr}^{90}$. This may suggest that there is something quite different about negative parity and positive parity transitions; however, the differences are not so large as to allow an unambiguous conclusion. Calculations with exchange treated exactly are needed to see exact 1 y how big these differences are. Also the $\mathrm{M} 4 \mathrm{\gamma}$-transition rate must be calculated as a check on the $L=3$ component of the cross section, although the shape agreement between theory and experiment at $61.4 \mathrm{MeV}$ suggests that it is given fairly well.

\section{Microscopic Empirical Formula}

For a normal parity transition the microscopic empirical formula of Atkinson and Madsen, Eq. (D.63), provides a relationship between the enhancement due to core polarization, $\varepsilon$, of a valence transition in the $\left(p, p^{\prime}\right)$ reaction and the nature of the effective charge. For a transition involving valence protons Eq. (D.63) is conveniently rewritten as

$$
\varepsilon_{p}=f_{p}+\alpha f_{n}
$$


where $f_{p}=e p$, is the observed effective charge, which is given by the ratio of the total proton transition density to the valence proton transition density; $f_{n}$ is the ratio of the neutron core transition density to the valence proton transition density; and $\alpha$ is the ratio of the strength of the $n-p$ force to the $p-p$ force. For the $K-K$ force $\alpha$ is about 2.5 . The effective charge gives a measure of the enhancement of a $\gamma$-transition rate due to core polarization. It is clear that the corresponding enhancement factor for the $\left(p, p^{\prime}\right)$ reaction should be much larger than the effective charge if $f_{n}$. is comparable to $e_{p}$. This is simply a result of the fact that the $K-K$ force gives more weight to neutron excitations than proton excitations in the $\left(p, p^{\prime}\right)$ reaction.

When the valence particles are neutrons Eq. (D.63) can be written

$$
\varepsilon_{n}=f_{n}+f_{p} / \alpha
$$

Now $f_{p}=e_{n}$, is the effective charge, which is given by the ratio of the proton core transition density to the valence neutron transition density and $f_{n}$ is the ratio of the total neutron transition density to the valence neutron transition density. The fact that proton excitations are given 2.5 times less weight than neutron excitations in the ( $\left.p, p^{\prime}\right)$ reaction is again clearly displayed in the formula. For fixed $f_{p}$ and $f_{n}$ the enhancement factors for the case of valence will be much smaller than for the case of valence protons. This occurs because a large weight has been assigned to the valence transition when the valence particles are neutrons. 
The smaller enhancement factors for neutron valence particles, as compared to proton valence particles, do not imply smaller core polarization effects.

The iso-scalar and iso-vector effective charges are related to $f_{p}$ and $f_{n}$ by:

$$
\begin{aligned}
& e_{0_{1}}=f_{p} \pm f_{n} \text { for proton valence particles } \\
& e_{0_{j}}=f_{n} \pm f_{p} \text { for neutron valence particles }
\end{aligned}
$$

An iso-scalar transition corresponds to the condition $f_{p}=f_{n}$ which is equivalent to $e_{1}=0$. Transitions with iso-scalar core excitation are defined by $\mathrm{f}_{\mathrm{p}_{\mathrm{n}}}^{\mathrm{f}}=\underset{\mathrm{p}_{\mathrm{n}}}{\mathrm{f}_{\mathrm{n}^{\prime}}+l}$ which is the same as $e_{1}=1$. For proton valence particles and fixed $e_{p}$, the iso-scalar condition implies larger values of $\varepsilon_{p}$ than does the condition of iso-scalar core excitation, i.e. a larger neutron core transition density is implied by the first condition. For neutron valence particles and fixed $e_{n}$, the condition of iso-scalar core excitation implies a larger neutron core transition density and a larger $\varepsilon_{n}$ than does the iso-scalar condition. Both of these conditions imply strong correlations between protons and neutrons when core polarization is large. Whenever there is a great deal of core polarization the differences between the conditions will not be very significant. 
The experimental relationship between $\varepsilon_{p}\left(\varepsilon_{n}\right)$ and $e_{p}\left(e_{n}\right)$ for the lowest quadrupole transitions in $\mathrm{Zr}^{90}(\mathrm{~A}), \mathrm{Ti}^{50}(\mathrm{~B})$, $\mathrm{Ni}^{58}(\mathrm{C})$, and $\mathrm{Pb}^{207}(\mathrm{D})$ is shown in Fig. 10. Values of $\varepsilon_{\mathrm{p}}\left(\varepsilon_{n}\right)$ and $e_{p}\left(e_{n}\right)$ which lie within the boxes drawn in the figure are consistent with the experimental data. The experimental data for $\mathrm{Zr}^{90}$ and $\mathrm{Ti}^{50}$ has been discussed previously. The lower limit on $\varepsilon_{p}$ for these two transitions are the results of this work, $\mathrm{i} . e$. they have been obtained from the $\mathrm{K}-\mathrm{K}$ force with exchange treated approximately: The upper limit on $\varepsilon_{\mathrm{p}}$ for $\mathrm{Zr}^{90}$ is obtained from the results of Love, Satchler, and collaborators 67,74 for the $\mathrm{H}-J$ force with exchange treated exactly. The intermediate value of $\varepsilon_{\mathrm{p}}$ for $\mathrm{Zr}^{90}$ (indicated by the horizontal line through the middle of the box) are the results for the $K-K$ force, modified to correct for the deficiencies in the approximate treatment of exchange. This was also discussed previously. The upper and intermediate values of $\varepsilon_{\mathrm{p}}$ for $\mathrm{Ti}^{50}$ are estimates based on the corresponding $\mathrm{Zr}^{90}$ results.

$\mathrm{Ni}^{58}$ and $\mathrm{Pb}^{207}$ are two other nuclei which have been considered in the course of this investigation. They have not been discussed in this paper. The $\mathrm{Pb}^{207}$ results have been reported elsewhere. ${ }^{117} \mathrm{Ni}^{58}$ has been discussed by Zamick and Federman. ${ }^{88}$ Both of these nuclei have valence neutrons. The transition in $\mathrm{Ni}^{58}$ is from the $\mathrm{O}^{+}$ground state to the $2^{+}$state at $1.33 \mathrm{MeV}$ and the transition in $\mathrm{Pb}^{207}$ is the $3 p_{1 / 2}-2 f_{5 / 2}(Q=-.570 \mathrm{MeV})$ neutron-hole transition. The effective charges for $\mathrm{Ni}^{58}$ and $\mathrm{Pb}^{207}$ come from Ref. 88 and 


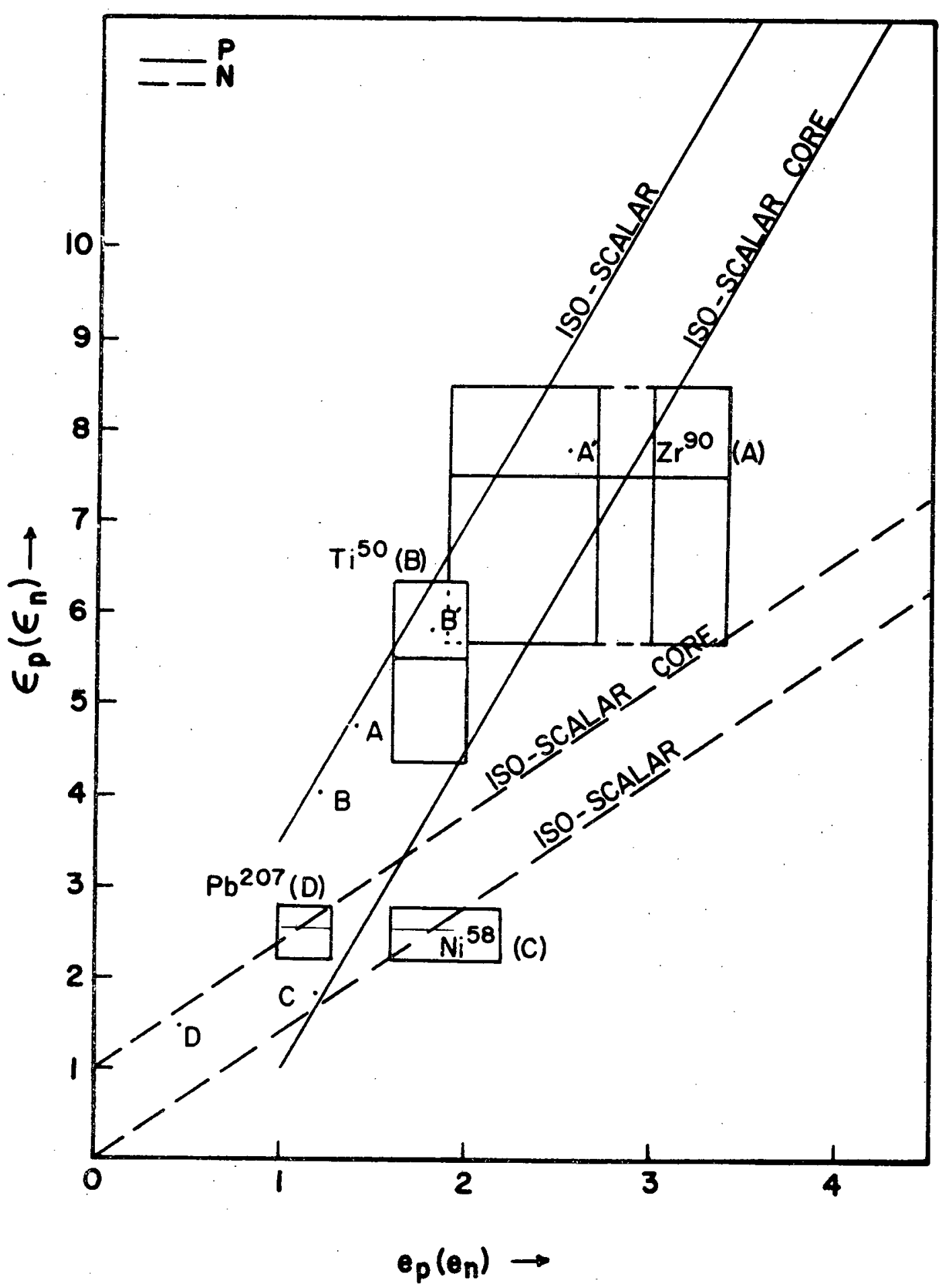

Figure 10.--Experimental relationship between $\varepsilon_{p}\left(\varepsilon_{n}\right)$ and $e_{p}\left(e_{n}\right)$ for quadrupole transitions in $\mathrm{Zr} 90(\mathrm{~A})$, Ti $50(\mathrm{~B}), \mathrm{Ni} 58(\mathrm{C})$, afid $\mathrm{Pb} 207(\mathrm{D})$. Results of theoretical calculations are also
shown. 
Ref. 94, respectively. The experimental ( $\left.p, p^{\prime}\right)$ cross sections for $\mathrm{Pb}^{207}$ comes from Ref. 107 while that for $\mathrm{Ni}^{58}$ comes from Ref. 5. The lower limits on $\varepsilon_{n}$ are again the results of this work and the upper and intermediate values on $\varepsilon_{n}$ for $\mathrm{Pb}^{207}$ are based on the results of Ref. 67. The upper and intermediate values of $e_{n}$ for $\mathrm{Ni}^{58}$ are only estimates. Also shown in Fig. 10 are lines corresponding to the iso-scalar condition and the condition of iso-scalar core excitation. The solid lines are for valence protons and the dashed lines are for valence neutrons. Observe that above the iso-scalar line you have more neutron excitation than proton excitation in the transition. Below the iso-scalar line this situation is reversed.

The experimental data is not terribly definitive, but the boxes definitely tend to stay somewhere in the vicinity of the iso-scalar and the iso-scalar core lines, i.e. the data implies that there are strong correlations between proton and neutron excitations in these transitions. For $\mathrm{Zr}^{90}$, $\mathrm{Ti}^{50}$, and $\mathrm{Ni}^{58}$ the data says that the total proton transition density is equal to or greater than the total neutron transition density. This is consistent with the findings of Schaeffer ${ }^{118}$ who has studied the $\left(p, p^{\prime}\right)$ data and the $\gamma$-decay data for the first $2^{+}$and $3^{-}$excitations in $\mathrm{Sr}^{88}, \mathrm{Zr}^{90}$, and the $\mathrm{Ni}$ isotopes. For $\mathrm{Pb}^{207}$ the data implies more neutron excitation than proton excitation. It should also be pointed out that the results shown here are not inconsistent with proton and neutron excitation in the ratio $\mathrm{Z} / \mathrm{N}$ which has been 
suggested from comparitive studies of the $\left(\alpha, \alpha^{\prime}\right)$ data and $\gamma$-transition rates. 119 For $\mathrm{Zr}^{90}, \mathrm{Ti}^{50}$, and $\mathrm{Ni}^{58}$ the $\mathrm{Z} / \mathrm{N}$ condition is not too different from the iso-scalar condition and for $\mathrm{Pb}^{207}$ it implies quite a bit more neutron excitation than proton excitation.

The data favors the iso-scalar condition for $\mathrm{Ni}^{58}$ and the condition of iso-scalar core excitation for $\mathrm{Pb}^{207}$. For $\mathrm{Zr}^{90}$ and $\mathrm{Ti}^{50}$ it is difficult to distinguish between the two conditions from the data. The lower limits on $\varepsilon_{p}$ imply that iso-scalar core excitation is required. The upper limits on $\varepsilon_{\mathrm{p}}$ favor the iso-scalar condition. In reaching this conclusion the higher value of $e_{p}$ for $\mathrm{zr}^{90}$ has been considered suspicious. This is admittedly arbitrary. Recent experimental data on quadrupole $\gamma$-transition rates in $\mathrm{Ca}^{42}$ and $\mathrm{Ti}^{50}$ indicates that iso-scalar core excitation is favored in the $1 f_{7 / 2}$ shell. ${ }^{120}$ The results presented here are consistent with this finding, but they do not substantiate it.

In conjunction with Fig. 10, experimental values of $f_{p}$ and $f_{n}$ for these transitions are presented in Table 10 . 'To sets of values are given for each transition--one for the upper limits on $\varepsilon_{p}$ and $\varepsilon_{n}$ and one for the lower limits. They are labeled $\varepsilon_{>}$and $\varepsilon_{<}$, respectively.

The results of the particle-hole calculations for $\mathrm{Zr}^{90}, \mathrm{Ti}^{50}, \mathrm{Ni}^{58}$, and $\mathrm{Pb}^{207}$ are also given in Fig. 10 and Table 10. In the figure these results are indicated by the points A, B, C, and D, respectively. For $\mathrm{Zr}^{90}, \mathrm{Ti}^{50}$, and 
TABLE 10.--Experimental and theoretical values for the normalized proton and neutron transition densities for quadrupole transitions in $\mathrm{Zr} 90$, Ti50, $\mathrm{Ni} 59$, and $\mathrm{Pb}^{20}$ ?

\begin{tabular}{|c|c|c|c|c|c|c|}
\hline \multirow{3}{*}{ Nucleus } & \multicolumn{4}{|c|}{ Experiment } & \multirow{2}{*}{\multicolumn{2}{|c|}{ Theory }} \\
\hline & \multicolumn{2}{|c|}{$\varepsilon_{>}$} & \multicolumn{2}{|c|}{$\varepsilon_{<}$} & & \\
\hline & $f_{p}$ & $f_{n}$ & $f_{p}$ & $f_{n}$ & $f_{p}$ & $f_{n}$ \\
\hline $\mathrm{Zr}^{90}$ & $2 \cdot 30$ & $2 \cdot 30$ & $2 \cdot 30$ & 1.30 & 1.41 & $1.34^{\mathrm{a}}$ \\
\hline & & & & & 2.55 & $2.11^{\mathrm{b}}$ \\
\hline $\mathrm{Ti}^{50}$ & 1.80 & 1.80 & 1.80 & 0.80 & 1.22 & $1.14^{\mathrm{a}}$ \\
\hline & & & & & 1.81 & $1.61^{b}$ \\
\hline $\mathrm{Ni}{ }^{58}$ & 1.90 & 1.90 & 1.90 & 1.90 & 1.20 & $1.40^{\mathrm{a}}$ \\
\hline $\mathrm{Pb}^{207}$ & 2.13 & 1.13 & 2.13 & 1.13 & $1 \cdot 30$ & $0.45^{\mathrm{a}}$ \\
\hline
\end{tabular}

$\mathrm{Ni}^{58}$ the results for the particle-hole model fall very near the iso-scalar lines. For $\mathrm{Pb}^{207}$ the particle-hole model gives a result near the iso-scalar core line. ${ }^{\dagger}$ In all cases

$t_{\text {The }}$ reader is warned not to attach too much significance to this particular result. For $\mathrm{Zr} 90$ and Ti50 the particlehole model predicts much larger neytron core excitation than proton core excitation and for $\mathrm{Ni} 58$ there is much more proton core excitation then neutron core excitation, i.e. valence protons couple more strongly to core neutrons and valence neutrons couple more strongly to core protons. The small ratio of proton core excitations to neutron core excitations for $\mathrm{Pb} 207$ is a result of the fact that the same harmonic oscillator well was used for neutron and proton single particle orbitals. This is tantamount to assuming there is neutron skin for which there is no experimental evidence. The proton and neutron wells probably should be adjusted so that the low lying proton particle and hole orbitals have radii comparable to the valence neutron orbitals. This will improve the overlap between the low lying proton orbitals and the valence neutron orbitals and a larger contribution from proton core excitations will result. 
the particle-hole model underestimates both the enhancement factor and the effective charge. From Table 10 it is clear that the particle-hole model does not do too badly for the neutron core transition density when the valence particles are protons, but it tends to underestimate the proton core transition density by a fairly large factor. For the case of valence neutrons the model does fairly well for the proton core transition density and tends to underestimate the neutron core transition by a substantial factor. This simply bears out what was said earlier, i.e. the neutron-proton imbalance in the core transition densities, which is predicted by the particle-hole model, is not consistent with experiment.

It is not too bothersome that the particle-hole calculations do note produce perfect agreement with the experimental transition rates. It definitely gives a good qualitative estimate of the overall effect of core polarization. It has already been pointed out that it doesn't do a perfect job for the spectrum, and that the question of fairly strong, low lying core phonons cannot be ignored. Further, the coupling between the valence particles and the core is a little too strong (e.g. see amplitudes in Tables 2,3 and 5 of this chapter) to allow one to take first order perturbation theory too seriously. The results of Kirson and Barret ${ }^{121}$ do, in fact, demonstrate that the perturbation series for the spectrum converges only slowly, if at all. 
It is interesting to follow up on a suggestion due to Harvey ${ }^{122}$ to see if the results of the particle-hole calculation can be improved in a simple way. He points out that Horie and Arima 57 did not use the "bare" force (the $\mathrm{K}-\mathrm{K}$ force in this work) in calculating quadrupole moments within the framework of the particle-hole model. Instead they used a two-body force which was fit to the experimental spectrum, i.e. a renormalized force in our language. He argues that this procedure might give a much better estimate of effective transition operators than does the first order perturbative calculation using the "bare" force. Just how good this new estimate is depends on just how well the actual renormalized force, which is a complicated operator, can be represented by a two-body force determined from the spectrum.

A calculation using this approach was made for the $\mathrm{L}=2-8$ transitions in $\mathrm{Zr}^{90}$ and the $\mathrm{L}=2-6$ transitions in $\mathrm{Ti}^{50}$. The renormalized force was taken to be of the form

$$
\bar{V}=V+G 3 p-I h
$$

where $V$ denotes the $K-K$ force and $G_{3 p-I h}$ was taken to be separable, i.e.

$$
G_{3 p-l h}=-k_{V}(r) k_{V}\left(r^{\prime}\right) \delta_{T I} \sum_{L} \theta_{L} Y_{L}^{*}(\hat{r}) \cdot Y_{L}\left(\hat{r}^{\prime}\right)
$$

The $\theta_{L}$ are given in Table 1 of this chapter. The additional assumption is made that $G_{3 p-l h}$ only acts in $T=1$ states. 
Spectra typically require large renormalizations of the bare force only in $\mathrm{T}=1$ states. 20,27

The results obtained for the quadrupole transition rates in $\mathrm{Zr}^{90}$ and $\mathrm{Ti}^{50}$ are shown in Fig. 10. They are labeled $\mathrm{A}^{\prime}$ and $B^{\prime}$, respectively. The corresponding values of $f_{p}$ and $f_{n}$ are compared with the experimental values in Table 10.

Table 11 gives a breakdown of the results for all the multipoles in $\mathrm{Zr}^{90}$ and $\mathrm{Ti}^{50}$ and comparison is made with the results of the perturbative calculation. Theoretical enhancement factors are also compared with the experimental values.

TABLE 11.--Normalized proton and neutron transition densities as given by the particle-hole model and particle-hole model with renormalized rurce for $L=2-8$ transitions in $\mathrm{Zr} 90$ and for $\mathrm{I}=2-6$ transitions in Ti50. Theoretical and experimental enhancement factors are also shown: For $\mathrm{Zr} 90$ the experimental $\varepsilon$, values are from Ref. 67 . The Ti50 $\varepsilon_{>}$values are estimates.

\begin{tabular}{|c|c|c|c|c|c|c|c|c|c|}
\hline \multirow[t]{2}{*}{ Nucleus } & \multirow[t]{2}{*}{$L$} & \multicolumn{3}{|c|}{$\mathrm{p}-\mathrm{h}$ Model } & \multicolumn{3}{|c|}{ Renorm. Force } & \multicolumn{2}{|c|}{ Experiment } \\
\hline & & $f_{p}$ & $\mathrm{f}_{\mathrm{n}}$ & $\varepsilon_{p}$ & $f_{p}$ & $f_{n}$ & $\varepsilon_{p}$ & $\varepsilon_{>}$ & $\varepsilon_{<}$ \\
\hline \multirow{4}{*}{$\mathrm{zr}^{90}$} & 2 & 1.41 & 1.34 & $4 \cdot 35$ & 2.55 & 2.11 & 7.83 & 8.51 & 5.80 \\
\hline & 4 & 1.25 & 1.06 & 3.56 & 1.62 & $1 \cdot 37$ & 5.05 & 7.45 & 4.52 \\
\hline & 6 & 1.14 & 0.84 & 3.09 & 1.30 & 0.95 & 3.68 & 6.19 & 4.52 \\
\hline & 8 & 1.08 & 0.60 & 2.58 & 1.16 & 0.61 & 2.69 & 6.30 & 7.48 \\
\hline \multirow{3}{*}{$\mathrm{Ti}^{50}$} & 2 & 1.22 & 1.14 & 4.24 & 1.81 & 1.61 & 5.84 & 6.35 & 4.36 \\
\hline & 4 & 1.16 & 0.92 & 3.74 & 1.40 & 1.11 & 4.18 & 6.26 & $4 \cdot 32$ \\
\hline & 6 & 1.07 & 0.63 & 2.65 & 1.18 & 0.70 & 2.93 & -- & -- \\
\hline
\end{tabular}


The agreement between theory and experimental for the $\mathrm{L}=2$ transitions is quite good. Both the proton and neutron core transition densities have been increased as compared to the perturbative results. The proton transition densities have gone through the largest relative change. Differences between the perturbative results and the results of the calculations with the renormalized force decrease with increasing multipole. The renormalized force gives a fairly hefty boost to $\varepsilon_{p}$ for the $L=4$ transitions and it produces a sizeable increase in the polarization charge for all multipoles. It is difficult to discuss the multipole dependence of $\varepsilon_{p}$ because of the fairly large uncertainties in the experimental values, i.e. $\varepsilon_{>}$and $\varepsilon_{<}$bracket a fairly large range of values. It would be useful to have $\left(e, e^{\prime}\right)$ data for these transitions as it would provide information on the multipole dependence of the effective charge. In any event this procedure would appear to have some merit. The calculation reported here is quite rough and a more careful investigation of this approach is planned. 


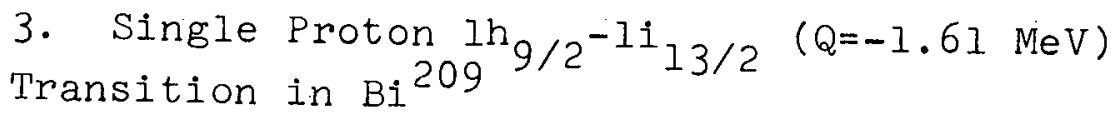

The nucleus $\mathrm{Bi}^{209}$ has one proton outside a $\mathrm{Pb}^{208}$ core. The valence proton is in the $1 h_{9 / 2}$ orbit for the ground state of this nucleus. The first excited state $(Q=-1.609 \mathrm{MeV})$ has the valence proton in the $1 i_{13 / 2}$ level. Twenty triads (LSJ) contribute to the transition between these two levels. The two most important ones are expected to be (112) and (303). This is similar to the situation for the single proton transition in $Y^{89}$ which has just been treated. One might expect these two states to be connected by an M2 $\gamma$-transition. In exciting the $I_{13 / 2}$ level in the ( $\left.p, p^{\prime}\right)$ reaction one might expect to observe a differential cross section which is composed of (112) and (303) components in analogy with $\mathrm{Y}^{89}$.

Contrary to these expectations, the $1.609 \mathrm{MeV}$ is observed to decay to the ground state by an $\mathrm{E} 3 \gamma$-transition with $B(E 3)=(1.3-2.0) \times 10^{-2} e^{2} b^{3} \cdot 110,111$ The core nucleus, $\mathrm{Pb}^{208}$, has a highly collective $3^{-}$state at $2.614 \mathrm{MeV}$. This phonon is quite stable as a closely spaced septet of states are observed in $\mathrm{Bi}^{209}$ at roughly $2.6 \mathrm{MeV}$. The septet results from the coupling of the $1 h_{9 / 2}$ proton to the $3^{-}$phonon of $\mathrm{Pb}^{208}$. Another septet, formed by coupling a $1 i_{13 / 2}$ proton to this same state, is expected at about $4.2 \mathrm{MeV}$. This is to be contrasted with the situation in $\mathrm{Y}^{89}$ where no strong $5^{-}$state is observed in the spectrum of the core nucleus, $\mathrm{Sr}^{88}$. 
The $\mathrm{Pb}^{208}\left(\mathrm{He}^{3}, \mathrm{~d}\right) \mathrm{Bi}^{209}$ experiment has been performed ${ }^{112}$ and some $\left(\mathrm{He}^{3}, \mathrm{~d}\right)$ strength is observed in the $13 / 2^{+}$member of the septet at $2.602 \mathrm{MeV}$. Using the particle-vibration coupling model Mottleson 113 has estimated the mixing of the first two $13 / 2^{+}$states in $\mathrm{Bi}^{209}$. The admixture of the $2.602 \mathrm{MeV}$ state into the $1.609 \mathrm{MeV}$ state is $\varepsilon^{2}=4.8 \times 10^{-2}$. In this calculation the coupling matrix element was obtained from the $\gamma$-decay of the $3^{-}$state of $\mathrm{Pb}^{208}$. The mixing of the states accounts for the observed ( $\left.\mathrm{He}^{3}, \mathrm{~d}\right)$ strengths. The $1.609 \mathrm{MeV}$ state of $\mathrm{Bi}^{209}$ has been excited in the $\left(p, p^{\prime}\right)$ reaction at $39.5 \mathrm{MeV}$ and a differential cross section is available. 114 Following Kuo's suggestion 90 that the particle-hole treatment of core polarization may not be adequate when there is the possibility of contributions from highly collective phonons of the core (which appears to be the case for this transition) the cross section is calculated in two ways: ( 1 ) including only $2 p$-lh components in the wave functions, and (2) replacing the components with $\mathrm{p}-\mathrm{h}$ coupled to angular momentum $\mathrm{J}_{\mathrm{c}}=3$ by components which contain the $3^{-}$ core state of ${ }^{208} \mathrm{~Pb}$. In the latter calculation the macroscopic vibrational model is used to describe the core. The wave functions corresponding to calculation ( 1 ) will be designated Set I while those corresponding to calculations (2) will be called set II.

Particle-hole pairs are formed from the shells shown in Table 12. Harmonic-oscillator wave functions have been used, and the energy denominators were taken in part from 
TABLE 12 --Particle and hole orbitals used in microscopic

calculation. The absence of total angular-momentum subscript indicates that both $j=l \pm 1 / 2$ orbits are included.

\begin{tabular}{|c|c|c|c|}
\hline \multicolumn{2}{|c|}{ Particles } & \multicolumn{2}{|c|}{ Holes } \\
\hline Protons & Neutrons & Protons & Neutrons \\
\hline $\ln _{9 / 2}$ & $1 i_{11 / 2}$ & $1 d$ & lf \\
\hline $2 f$ & $2 g$ & $2 \mathrm{~s}$ & $2 p$ \\
\hline $3 p$ & $3 d$ & If & $\lg$ \\
\hline $1 i$ & $4 s$ & $2 p$ & $2 d$ \\
\hline $2 g$ & $I j$ & $1 g$ & $3 s$ \\
\hline $3 d$ & $2 \mathrm{~h}$ & $2 d$ & In \\
\hline $4 s$ & $3 f_{7 / 2}$ & $3 s$ & $2 f$ \\
\hline $1 j_{15 / 2}$ & & $.1 \mathrm{~h}_{11 / 2}$ & $3 p$ \\
\hline${ }^{2} \mathrm{~h}_{11 / 2}$ & & & ${ }^{I i_{13 / 2}}$ \\
\hline
\end{tabular}

experiment ${ }^{115}$ and in part from the Nilsson scheme at zero deformation. The size parameter $h \omega$ is $6.8 \mathrm{MeV}$.

Ref. 113 gives $\left\langle\mathrm{k}_{\mathrm{v}}>=60 \mathrm{MeV}\right.$ and $\mathrm{C}_{3}=649 \mathrm{MeV}$. Analysis of the reaction ${ }^{208} \mathrm{~Pb}\left(\mathrm{p}, \mathrm{p}^{\prime}\right)^{208} \mathrm{~Pb}$ gives $\beta_{3} \sim 0.13$ for this state 77,85,116 which is the only state with a large value of $\beta$ in ${ }^{208} \mathrm{~Pb}$. The relation $\beta_{3}=7^{1 / 2}\left(x \omega_{2} / 2 C_{3}\right)^{1 / 2}$ implies $\mathrm{C}_{3}=543 \mathrm{MeV}$ which is smaller than the value from Ref. 113 and corresponds to an admixture $\varepsilon^{2}=5.5 \times 10^{-2}$ of the $2.602-\mathrm{MeV}$, $\frac{13+}{2}$ state in the $1.609-\mathrm{MeV}, \frac{13+}{2}$ state. The smaller value of $\mathrm{C}_{3}$ is used in this work. 
In these calculations, as a matter of convenience, a pseudo-potential has been used for the projectile-target interaction. This pseudo-potential is known to give results consistent with those obtained using the $K-K$ force and treating antisymmetrization approximately. The $2 \mathrm{p}-1 \mathrm{~h}$ components of the cross section have been included only in the $\dot{S}=0$ terms in the cross section because it is only in these components that they add coherently. In using wave function Set II the components of the wave functions containing the core phonon contribute only to the $(\mathrm{LSJ})=(303)$ component of the cross section. The remaining 19 components are the same in Sets $I$ and II.

Figure 11 shows the total differential cross sections obtained with wave function set I and Set II. The (303) components are also shown for both cases. The differential cross section (II) gives a good fit to the experimental data. The (303) (II) component is dominant as forward angles. The enhancement due to core polarization, of (303) (II) is about 200. Because of this large enhancement the valence contribution to (303) (II) is small. Considering only this component and neglecting the valence contribution, the data places an upper limit on $\varepsilon^{2}=10^{-1}$. Wave function set II gives $B(E 3)-2.4 \times 10^{-2} e^{2} b^{3}$ which is slightly larger than the experimental values.

The particle-hole model fails to reproduce the effect of the $3^{-}$phonon of ${ }^{208} \mathrm{~Pb}$. The enhancement of (303) (I) is 


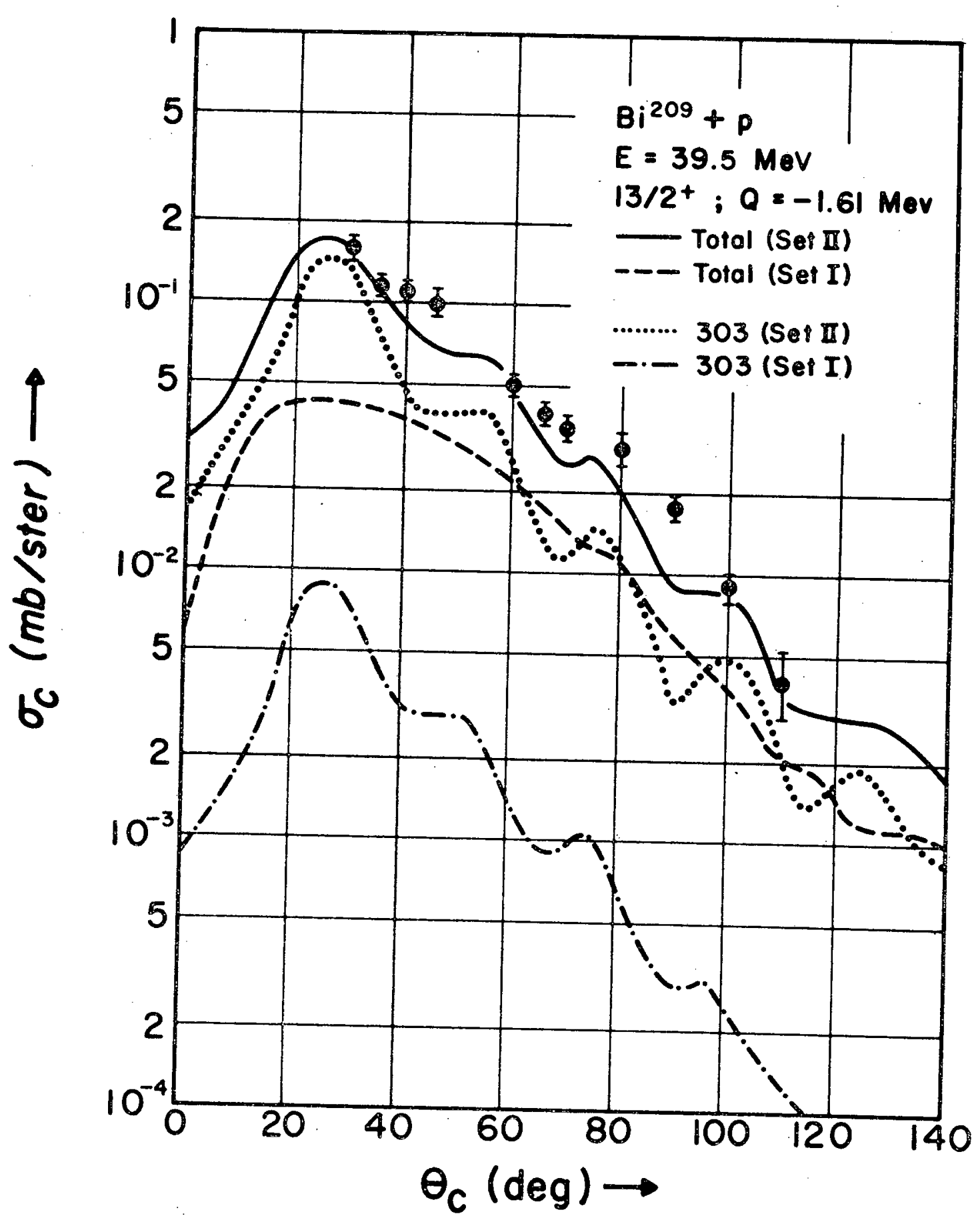

Figure 11.--The experimental data compared with the theoretical results obtained with both sets of wave functions. The total differential cross sections and the (303) component are shown for both cases. 
about 13 which is an order of magnitude smaller than the value obtained for (303) (II). This model predicts that many components make important contributions to the total differential cross section. In particular, (303) (I) is comparable in magnitude with (112) which involves the lowest allowed $\mathrm{L}$ and $\mathrm{J}$ transfers. As the lowest $\mathrm{J}$ transfer is highly favored in $\gamma$-transitions, the particle-hole model predicts that the $1.609 \mathrm{MeV}, \frac{13+}{2}$ state will decay to the ground state predominantly by an Ma transition which is in contradiction to experiment.

It is concluded that highly collective core phonons can play an extremely important part in the core polarization process. This is another indication that care must be exercised in applying the uncorrelated particle-hole model for core polarization.

NOTE added in proof: A calculation was performed to estimate the effect of exciting proton particles from the core into the valence orbitals in $\mathrm{Zr}^{90}$ and $\mathrm{Ti}^{50}$. These excitations were treated the same as proton excitations into orbitals outside the valence space, but amplitudes of configurations with three particles in the same orbit were multiplied by $(n-2) / n$ (where $n=2 j+1$ ) to account for violations of the Pauli principle. Experimentally observed single particle energy denominators were used in the calculation. With these excitations included $\mathrm{e}_{\text {eff }}=1.41,1.26,1.14$, and 1.08 for $\mathrm{L}=2-8$ in $\mathrm{Zr}^{90}$ and 
$\mathrm{e}_{\text {eff }}=1.22,1.16$, and 1.07 for $\mathrm{L}=2-6$ in $\mathrm{Ti}^{50}$. These are not much different than the results shown in Table 9 of this chapter. The result for the $\mathrm{L}=2$ transition in $\mathrm{Zr}^{90}$ shows the biggest change. Here quite a large contribution was obtained from the $2 p_{1 / 2}-2 p_{3 / 2}$ proton particle-hole pair. These changes will not effect the $\left(p, p^{\prime}\right)$ cross sections very much as they are primarily sensitive to the neutron excitations.

Note that the values for e eff (Macro) are somewhat larger than those for $\bar{e}_{\text {eff }}$ even if the effect of the above excitations are included. The assumption of the collective model is that the charge transition density is $z / A$ times the mass transition density. Thus one expects that $\bar{e}_{\text {eff }}$ should be slightly larger than eff(Macro). Coupling this to the fact that Ref. 15 gives $R_{c}^{2} /<r^{2}>>1$ for $Z r^{90}$ again suggests that a larger value of $\left\langle\mathrm{k}_{\mathrm{v}}\right\rangle$ than $50 \mathrm{MeV}$ should be used in these calculations. 


\section{CHAPTER 8}

\section{SUMMARY AND CONCLUSIONS}

It is felt that the results of this work, which are admittedly rough, clearly demonstrate the feasibility of using "realistic interactions" in describing the inelastic scattering of 15-70 MeV nucleons from nuclei in a microscopic picture. The use of such interactions requires a fairly detailed description of the target nuclei and it is necessary to treat antisymmetrization. These two requirements are not objectionable as the former is precisely the motivating factor for the microscopic approach while the latter should yield useful information about the interaction as well as the nuclear wave functions.

Three interaction models have been considered in this work and the majority of the calculations which have been performed provide information only about the strong central components of these forces. The results obtained are sensitive to the gross features of the force, i.e. strength and range, and the impulse approximation pseudo-potential and the $\mathrm{K}-\mathrm{K}$ force appear to be somewhat better than the Yukawa effective range force. The first two contain information about the high momentum components of the free two nucleon force while the third does not. In work on the optical potential, 31,32 it was shown that the impulse approximation 
pseudo-potential does not have the correct phase--a property which is not examined in the inelastic scattering calculations-and that the $K-K$ force was better than the Yukawa effective range force.

A convenient approximate treatment of antisymmetrization has been developed and used in this work. This approximation has been shown to be qualitatively correct in general and gives good quantitive results for Yukawa forces of $1 F$ range at incident energies in excess of $40 \mathrm{MeV}$. For Yukawa forces of longer range and at lower energies the approximation is still fair, but it appears to be considerably poorer for the $K-K$ force. Although the $K-K$ force is favored theoretically, uncertainties due to this approximation make it difficult to say that it is better than the Yukawa effective range force solely on the basis of the inelastic scattering data.

Finally it has been shown that a simple perturbative treatment of the effects due to core polarization does quite well in explaining the observed differential cross sections for the excitation of low lying levels in several nuclei with one or two protons outside of a closed shell. Related effects such as the effective charge and the pairing contribution to the ground energy of such nuclei have also been considered. The models used in this work are found not to be correct in detail, but the results obtained are very encouraging and the use of inelastic nucleonnucleus scattering as a tool for studying these effects should prove to be informative. 


\section{REFERENCES}

218 


\section{REFERENCES}

1. G. E. Brown, Unified Theory of Nuclear Models and NucleonNucleon Forces (North-Holland Publishing Company, Amsterdam, The Netherlands, 1967), 2nd ed.

2. A. M. Lane, Nuclear Theory (W. A. Benjamin, Inc., New York, $1 9 6 4 \longdiv { \text { . } }$

3. G. R. Satchler, Nucl. Phys. 55(1964)l.

4. N. K. Glendenning and M. Veneroni, Phys. Rev. $144(1966)$ 839.

5. N. K. Glendenning, in Rendiconti S. I. F. Course XI, 1967 (Academic Press, New York, 1969) p. 332.

6. G. R. Satchler, Nucl. Phys. 77(1966)481.

7. V. A. Madsen, ivicl. Phys. $80(1966) 177$.

8. W. S. Gray, R. A. Kenefick, J. J. Kraushaar and G. R. Satchler, Phys. Rev. 142(1966)735.

9. M. B. Johnson, L. W. Owen, and G. R. Satchler, Phys. Rev. $142(1966) 748$.

10. M. M. Stautberg, J. J. Kraushaar, and.B. W. Ridley, Phys. Rev. 157(1967)977.

11. G. R. Satchler, Nucl. Phys. A95(1967)1.

12. P. J. Locard, S. M. Austin, and W. Benenson, Phys. Rev. Lett. $19(1967) 1141$.

13. C. Wong, J. D. Anderson, J. McClure, B. Pohl, V. A. Madsen, and F. Schmittroth, Phys. Rev. I60(1967)769.

14. S. M. Austin, P. J. Locard, W. Benenson, and G. M. Crawley, Phys. Rev. 176(1967)1277.

15. W. G. Love and G. R. Satchler, Nucl. Phys. A101(1967) 424 . 
16. W. G. Love, University of Tennessee thesis, 1968 (un published).

17. K. A. Amos, V. A. Madsen, and I. E. McCarthy, Nucl. Phys. A94 (1967)103.

18. J. Atkinson and V. A. Madsen, Phys. Rev. Lett. 21 (1968) 295.

19. J. Atkinson and V. A. Madsen, private communication.

20. T. T. S. Kuo and G. E. Brown, Nucl. Phys. 85(1966)40.

21. E. C. Halbert, Y. E. Kim, and T. T. S. Kuo, Phys. Lett. 20 (1966)657.

22. T. T. S. Kuo, Nucl. Phys. A90(1967)199.

23. G. E. Brown and T. T. S. Kuo, Nucl. Phys. A92(1967)481.

24. T. T. S. Kuo, Nucl. Phys. Al03(1967)71.

25. T. T. S. Kuo and G. E. Brown, Nucl. Phys. Al14(1968)241.

26. B. H. Brandow, Rev. Mod. Phys. 39(1967)771.

27. M. H. Macfarlane, in Rendicotti S. I. F. Course XL, 1967 (Academic Press, New York, 1969) p. 457.

28. M. Baranger, in Rendicotti S. I. F. Course XL, 1967 (Academic Press, New York, 1969) p. 511.

29. K. W. MeVoy and W. J. Romo, A126 (1969)161.

30. T. Hamada and I. D. Johnston, Nucl. Phys. 34(1962) 382 .

31. D. Slanina and H. McManus, Nucl. Phys. Ail6(1968)271.

32. D. Slanina, Michigan State University thesis, 1969 (unpublished).

33. A. K. Kerman, H. McManus, and R. M. Thaler, Annals of Phys. 8(1959)551.

34. K. M. Watson, Phys. Rev. $\underline{89}(1953) 1957$.

35. N. C. Francis and K. M. Watson, Phys. Rev. 92(1953)291.

36. G. Takeda and K. M. Watson, Phys. Rev. 97(1955)1336. 
37. K. M. Watson, Rev. Mod. Phys. 30(1958)565.

38. M. L. Goldberger and K. M. Watson, Collision Theory (John Wiley and Sons, Inc., New York, 1964).

39. D. J. Jackson, Nucl. Phys. 35(1962)195.

40. R. M. Haybron and H. McManus, Phys. Rev. 136B(1964)1730.

41. R. M. Haybron and H. McManus, Phys. Rev. 140B(1965)638.

42. H. K. Lee, Michigan State University thesis, 1966 (unpublished).

43. H. K. Lee and H. McManus, Phys. Rev. 161(1967)1087.

44. H. K. Lee and H. McManus, Phys. Rev. Lett. 20(1968) 337.

45. K. Yagi et al., Phys. Lett. 10(1964)186.

46. A. Kallio and K. Kolltveit, Nucl. Phys. 53(1964)87.

47. S. A. Moskowski and B. L. Scott, Annals of Phys. 11
$(1960) 65$.

18. M. A. Preston, Physics of the Nucleus (Addison-wesle: Publishing Company, Reading, Massachusetts, 1962).

49. J. D. Jackson and J. M. Blatt, Rev. Mod. Phys. 22 (1950)

50. V. Gillet and N. Vinh Mau, Nucl. Phys. 54(1964)321.

51. V. Gillet and E. A. Sanderson, Nucl. Phys. 54 (1964)472.

52. V. Gillet, A. M. Green, and E. A. Sanderson, Nucl. Phys. 88(1967)321.

53. V. Gillet and E. A. Sanderson, Nucl. Phys. A9l(1967)292.

54. T. T. S. Kuo, unpublished results.

55. V. Gillet and M. A. Meikanoff, Phys. Rev. 133B(1964)1190.

56. T. deForest, Jr. and J. D. Walecka, Advances in Phys. 15(1966)1.

57. H. Horie and A. Arima, Phys. Rev. 99 (1955) 778.

58. D. M. Brink and G. R. Satchler, Angular Momentum (Oxford University Press, Oxford, Great Britain, 1962). 
59. D. M. Brink and G. R. Satchler, Nuovo Cim. 4 (1956)549.

60. B. P. Nigam, Phys. Rev. 133B(1964)1381.

61. R. H. Bassel, G. R. Satchler, R. M. Drisko and E. Rost, Phys. Rev. 128(1962)2693.

62. T. Tamura, Rev. Mod. Phys. 37(1965)679.

63. A. Bohr and B. R. Mottleson, Nuclear Structure: Volume I (W. A. Benjamin, Inc., New York, 1969) and unpublished notes.

64. L. J. Tassi, Aust. J. Phys. 9 (1956)407.

65. G. R. Satchler, Nucl. Phys. Al00(1967)481.

66. D. V. Grillot, Michigan State University thesis, 1967

67. G. R. Satchler, private communication and to be published.

68. A. M. Green, Phys. Lett. 24B(1967)384.

69. G. C. Ball and J. Cerny, Phys. Rev. 177(1969)1466.

70. W. W. True, Phys. Rev. 130(1963)1530.

71. W. R. Gibbs, V. A. Madsen, J. A. Miller, W. Tobocman, E. C. Cox, and L. Mowry, N. A. S. A. Report No. TND-2170 (unpublished).

72. E. L. Peterson, I. Slaus, J. W. Verba, R. F. Carlson, and J. Reginald Richardson, Nucl. Phys. Al02(1967)145.

73. S. M. Austin, P. J. Locard, S. N. Bunker, J. M. Cameron, J. Reginald Richardson, J. W. Verba, and W. T. H. van Oers, submitted to Phys. Rev.

74. W. G. Love, L. W. Owen, R. M. Drisko, G. R. Satchler, R. Stafford, and R. J. Philpott, submitted to. Phys.
Lett. 75. R. M. Haybron, M. B. Johnson, and R. J. Metzger,
Preprint.

76. P. J. Locard, private communication.

77. M. P. Fricke and G. R. Satchler, Phys. Rev. 139B(1965)

78. G. R. Satchler, Nucl. Phys. A92(1967)293. 
79. D. Agassi and R. Schaeffer, Phys. Lett. 26B(1968)703.

80. W. G. Love and L. J. Parish, to be published.

81. G. M. Crawley and S. M. Austin, in Proceedings of the International Nuclear Physics Conference, edited by R. L. Becker (Academic Press, New York, 1967)p. 165.

82. V. G. Neudatchin and Ya. F. Smirnov, in Progress in Nuclear Physics: Volume 10, edited by D. M. Brink and J. H. Mulvey (Pergamon Press, New York, 1969)

83. G. R. Hammerstein, unpublished results.

84. M. Bernheim and G. R. Bishop; Phys. Lett. 5(1963)270.

85. T. Stovall and N. M. Hintz, Phys. Rev. 135B(1964)330.

86. E. Baranger and C. W. Lee, Nucl. Phys. 22 (1961)157.

87. S. Gartenhaus and C. Schwartz, Phys. Rev. 108(1957)
482 .

88. P. Federman and L. Zamick, Phys. Rev. I77(1969)1534.

89. S. Siegel and L. Zamick, Phys. Lett. 28B(1969)450.

90. T. T. S. Kuo, Phys. Lett. 26B(1967)63.

91. S. Siegel and L. Zamick, Phys. Lett. 28B (1969)453.

92. A. K. Kerman, Phys. Rev. $\underline{92}$ (1953)1176.

93. K. W. Ford and C. Levinson, Phys. Rev. 100(1955)I.

94. W. W. True and K. W. Ford, Phys. Rev. 109(1958)1675.

95. B. F. Bayman, A. S. Reiner, and R. K. Sheline, Phys. Rev. I15(1959)1627.

96. S. Cohen, R. D. Lawson, M. H. Macfarlane, and M. Soga, Phys. Lett. 10(1964)195.

97. J. H. E. Mattauch, W. Thiele, and A. H. Wapstra, Nucl. Phys. 67(1965)I.

98. M. Goldhaber and A. W. Sunyar, Phys. Rev. 83(1951)
906.

99. K. K. Gupta and R. D. Lawson, Phys. Rev. 114(1959)326. 
100. H. O. Funsten, N. R. Roberson, and E. Rost, Phys. Rev. 134B (1964)117.

101. W. Benenson, S. M. Austin, R. A. Paddock, and W. G. Love, Phys. Rev. 176(1968)1268.

102. A. Scott, M. L. Whitten, and J. B. Ball, Phys, Lett. 25B $(1967) 463$.

103. R. J. Peterson, Annals of Phys. 53(1969)40.

104. M. P. Fricke, E. E. Gross, B. J. Morton, and A. Zucker, Phys. Rev. 156(1967)1207.

105. J. K. Dickens, E. Eichler, and G. R. Satchler, Phys. Rev. $168(1968) 1355$.

106. V. Madsen, private communication of result obtained from T. H. Curtis.

107. J. Vervier, Phys. Lett. 13(1964)47.

108. C. Glashausser et al., U.C.R.L. Report No. 18376(1968), submitted to Phys. Rev. Lett.

109. C. J. Maggiore, C. R. Gruhn, T. Y. T. Kuo, B. M. Preedom, private communication.

110. J. S. Lilley and W. R. Phillips, in John H. Williams Laboratory of Nuclear Physics, University of Minnesota, Annual Report No. COO 1265-67, 1968 (unpublished)p.123.

111. J. W. Hertel, D. G. Fleming, J. P. Schiffer, and H. E. Gove, Phys. Rev. Lett. 23(1969)488.

112. R. Woods, P. D. Barnes, E. R. Flynn, and G. J. Igo, Phys. Rev. Lett. 19(1967)453; J. S. Lilley and N. Stein, Phys. Rev. Lett. $\frac{19}{19}(1969) 709$; C. Ellegaard and P. Vedelsly, Phys. Lett. 26B(1968)155.

113. B. R. Mottleson, J. Phys. Soc. Japan Suppl. 24(1968)96.

114. W. Benenson, S. M. Austin, P. J. Locard, private communication.

115. D. A. Bromley and J. Weneser, Comments Nucl. Particle Phys. Il(1968)151.

116. G. R. Satchler, R. H. Bassel, and R. M. Drisko, Phys. Lett. 5(1963)256; A. Scott and M. P. Fricke, Phys. Lett. 20(1966)654; J. Sandinos; G. Vallois, O. Beer, M. Gendrot, and P. Lopato, Phys. Lett. 22(1966)492. 
117. H. McManus, J. R. Borysowicz, G. R. Hammerstein, W. Benenson, and F. Petrovich, contribution to International Conference on Properties of Nuclear States, Montreal, Canada (1969).

118. R. Schaeffer, Orsay thesis, 1969 (unpublished).

119. A. M. Bernstein, in Advances in Nuclear Physics: Volume 3, (Plenum Press, New York, 1969) p. 325.

120. T. Nomura, C. Gil, H. Saito, and T. Yamazaki, Phys. Rev. Lett. 25(1970)1342.

121. B. R. Barret and M. W. Kirson, Phys. Lett. 27B(1968) 544; Phys. Lett. 30B(1969)8; Nuc1. Phys. A148(1970) 145 .

122. M. Harvey, Chalk River Report, 1969 (unpublished). 


\section{APPENDICES}


APPROXIMATE SERIES FOR EXCHANGE COMPONENT OF D.W.A. TRANSITION AMPLITUDE

Expanding $t^{E}\left(\left|\bar{k}_{1}-\bar{k}_{2}\right|\right)$ in Eq. (2.5I) in a Taylor series about $\lambda_{0}^{2}$ keeping only the first two terms and then transforming back to a coordinate representation gives

$$
\begin{gathered}
I_{\text {ex }}=-\int x_{\mathrm{b}}^{(-) *}\left(\bar{r}_{0}\right) \phi_{\mathrm{p}}^{*}\left(\bar{r}_{1}\right)\{\} \delta\left(\bar{r}_{0 I}\right) \phi_{r}\left(\bar{r}_{0}\right) x_{a}^{(+)}\left(\bar{r}_{1}\right) d^{3} r_{0} d^{3} r_{1} \\
\{\}=A(I)\left(\lambda_{0}^{2}\right)-B\left(\lambda_{0}^{2}\right)\left(\nabla_{r_{0 I}}^{2}+\lambda_{0}^{2}\right)
\end{gathered}
$$

where $A^{(1)}\left(\lambda_{0}^{2}\right)$ is defined in Eq. (2.57) and

$$
B\left(\lambda_{0}^{2}\right)=\frac{d A(I)}{d\left(\lambda^{2}\right)} \mid \lambda^{2}=\lambda_{0}^{2}
$$

In Eq. (A.I) the $\nabla^{2}$ operator acts only on the $\delta$-function. The double integral (A.I) can be reduced to a single integral in two ways. One is to transform to an integral over $a^{3} r_{01} d^{3} r_{0}$ and integrate over $d^{3} r_{01}$. The other is to transform to an integral over $\mathrm{d}^{3} \mathrm{r}_{01} \mathrm{~d}^{3} \mathrm{r}_{1}$ and again integrate over $\mathrm{d}^{3} \mathrm{r}_{01}$. The results obtained can be used to write the single integral in the following symmetric form. 


$$
\begin{gathered}
I_{\text {ex }}=-\int x_{b}^{(-) *}\left(\bar{r}_{I}\right) \phi_{r}\left(\bar{r}_{I}\right)\{\} \phi_{p}\left(r_{I}\right) x_{a}^{(+)}\left(\bar{r}_{I}\right) d^{3} r_{I} \\
\{\}=A \\
(1)\left(\lambda_{0}^{2}\right)-B\left(\lambda_{0}^{2}\right)\left(\nabla^{2}+\lambda_{0}^{2}\right){ }_{\text {sym }} \\
\left(\nabla^{2}+\lambda_{0}^{2}\right)_{\text {sym }}=\frac{1}{2}\left[\left(\nabla^{2}+\lambda_{0}^{2}\right)+\left(\nabla^{2}+\lambda_{0}^{2}\right)\right]
\end{gathered}
$$

Some algebra, which involves performing the $\nabla^{2}$ operations in the integrand of Eq. (A.3), making use of the one body

Schrodinger equations which generate the $\chi^{\prime} s$ and $\phi^{\prime} s$, and performing a partial integration over one half of the resulting term which contains gradient operators, gives the following result for the exchange integral.

$$
\begin{aligned}
& I_{e x}=-\int x_{b}^{(-) *}\left(\bar{r}_{1}\right) \phi_{p}^{*}\left(\bar{r}_{1}\right) A\left(\lambda_{0}^{2} ; r_{1}\right) \phi_{r}\left(\bar{r}_{1}\right) x_{a}^{(+)}\left(\bar{r}_{1}\right) d^{3} r_{l} \\
& -B\left(\lambda_{0}^{2}\right) \int x_{\mathrm{b}}^{(-) *}\left(\bar{r}_{I}\right) \nabla \phi_{\mathrm{p}}^{*}\left(\bar{r}_{1}\right) \cdot \nabla \phi_{r}\left(\bar{r}_{I}\right) \chi_{\mathrm{a}}^{(+)}\left(\bar{r}_{I}\right) \mathrm{d}^{3} \mathrm{r}_{1} \\
& -\frac{1}{2} B\left(\lambda_{0}^{2}\right) \int \bar{J}(p, r) \cdot \bar{J}(b, a) d^{3} r_{1}
\end{aligned}
$$

In Eq. (A.4)

$$
\begin{gathered}
A\left(\lambda_{0}^{2} ; r_{1}\right)=A(I)\left(\lambda_{0}^{2}\right)-\left[\lambda_{0}^{2}-\frac{1}{2}\left(\kappa_{a}^{2}+\kappa_{b}^{2}\right)\right] B\left(\lambda_{0}^{2}\right) \\
k^{2}=k^{2}-\frac{2 \mu}{\hbar^{2}} U\left(r_{l}\right)
\end{gathered}
$$

where $U\left(r_{1}\right)$ is the optical potential and

$$
\begin{aligned}
& J(p, r)=\phi_{r}\left(\bar{r}_{I}\right) \nabla \phi_{p}^{*}\left(\bar{r}_{l}\right)-\phi_{p}^{*}\left(\bar{r}_{I}\right) \nabla \phi_{r}\left(\bar{r}_{I}\right) \\
& J(b, a)=\chi_{a}^{(+)}\left(\bar{r}_{l}\right) \nabla \chi_{b}^{(-) *}\left(\bar{r}_{1}\right)-\chi_{b}^{(-) *}\left(\bar{r}_{1}\right) \nabla \chi_{a}\left(\bar{r}_{1}\right)
\end{aligned}
$$


The first integral in Eq. (A.4) contains a dependence on the magnitude of the local momentum of the projectile and the second integral expresses dependence on the magnitude of the local momentum of the bound particle. These two integrals can be arranged to display the dependence on the momenta of the projectile and bound particle in a symmetric way; however, the form which is given is more convenient as it does not explicitly refer to the binding energy and potential of the bound particle. Both of these integrals can be easily handled in the local D.W.A.

The third integral in Eq. (A.4) cannot be incorporated conveniently in the local D.W.A. Contributions to non-normal transfer come from this term which essentially takes into account the fact that locally the projectile and bound particle are moving in different directions. The integral averages over these directions and the contributions for normal transfers are expected to be small. In the plane wave limit it can be shown that the integral vanishes for normal transfers when $\phi_{p}$ and $\phi_{r}$ are the same.

Neglecting the last term in Eq. (A.4) it follows that

$$
\tilde{E}^{L S J}\left(r_{0}\right)=\tilde{E}_{1}^{L S J}\left(r_{0}\right)+\tilde{E}_{2}^{L S J}\left(r_{0}\right)
$$

where $\tilde{E}_{I}^{L S J}\left(r_{0}\right)$ is given by Eq. (2.55) or Eq. (2.56) with the replacement $A^{(I)}\left(\lambda_{0}^{2}\right) \rightarrow A\left(\lambda_{0}^{2} ; r_{0}\right) \cdot \tilde{E}_{2}^{L S J}\left(r_{0}\right)$ contains the contribution from the second integral in Eq. (A.4). For the case of good i-spin 


$$
\begin{aligned}
& \tilde{E}_{2}^{L S J}\left(r_{0}\right)= \\
& \sum_{\mathrm{T}}^{j}-\sqrt{2} \hat{\mathrm{T}}<\mathrm{T}_{\mathrm{A}} \mathrm{TM}_{\mathrm{T}_{\mathrm{A}}}, \mathrm{M}_{\mathrm{T}_{\mathrm{B}}}-\mathrm{M}_{\mathrm{T}_{\mathrm{A}}}\left|\mathrm{T}_{\mathrm{B}} \mathrm{M}_{\mathrm{B}}><\frac{1}{2} \mathrm{~T} \tau_{\mathrm{b}}, \tau_{\mathrm{a}}-\tau_{\mathrm{b}}\right| \frac{1}{2} \tau{ }_{\mathrm{a}}> \\
& x i^{L+\ell-\ell^{\prime}} \sqrt{2} \hat{j} \hat{L S} \hat{S} \hat{J} \hat{T} \underline{x}\left(j ’ j J ; \ell / \ell L ; \frac{1}{2} \frac{1}{2} S\right) S\left(J_{A} J_{B} J ; T_{A} T_{B} T ; j j^{\prime}\right) \\
& x(4 \pi)^{-1 / 2} B_{S T}\left(\lambda_{0}^{2}\right) \mathcal{F}
\end{aligned}
$$

and when i-spin is ignored

$$
\begin{aligned}
& \tilde{\mathrm{E}}_{2}^{\mathrm{LSJ}}\left(r_{0}\right)= \\
& \sum_{j}-\sqrt{2} i^{L+\ell-\ell^{\prime}} \sqrt{2} \hat{j} \hat{L} \hat{S} \hat{J} \underline{\underline{X}}\left(j^{\prime} j J ; \ell^{\wedge} \ell L ; \frac{1}{2} \frac{1}{2} S\right) S\left(J_{A} J_{B} J ; j j^{\prime} \tau \tau^{\prime}\right) \\
& x(4 \pi)^{-1 / 2} B_{S \tau \tau}-\left(\lambda_{0}^{2}\right) F
\end{aligned}
$$

In these equations

$$
\begin{aligned}
& \mathcal{F}=\sum_{(+),(-)}(-I)^{L}() \pi_{+-} \hat{L} \dot{(} \hat{L}^{L}() u_{n}^{*}()\left(r_{0}\right) u_{n \ell}^{()}\left(r_{0}\right) \\
& x W\left(L_{(}()^{l L^{\prime}}()^{\left.\ell^{\prime} ; I L\right)}\left(\begin{array}{lll}
L^{\prime}()^{L}()^{L} \\
0 & 0 & 0
\end{array}\right)\right.
\end{aligned}
$$

where $\left(\begin{array}{l}a b c \\ \alpha \beta \gamma\end{array}\right)$ is a $3-j$ symbol, ${ }^{58} \mathrm{~L}_{( \pm)}^{\prime}=\ell^{-} \mp l, L_{( \pm)}=\ell \mp l$, and

$$
\begin{aligned}
& u_{n \ell}^{(+)}(r)=\ell^{I / 2}\left(\frac{d}{d r}+\frac{\ell+l}{r}\right) u_{n \ell}(r) \\
& u_{n \ell}^{(-)}(r)=(\ell+I)^{I / 2}\left(\frac{d}{d r}-\frac{\ell}{r}\right) u_{n \ell}(r)
\end{aligned}
$$


'l'here are four terms in the above sum and $\pi_{+-}$is a phase which is positive for the $(+)(+)$ and $(-)(-)$ terms and negative for the $(-)(+)$ and $(+)(-)$ terms. The net effect of including these additional terms in $\tilde{E}^{L S J}\left(r_{0}\right)$ is to damp out contributions to exchange scattering which come from the nuclear interior. This is reasonable as the momenta of the projectile and bound particle are much larger in this region than they are outside the nucleus. The exchange scattering here should sample momentum components of the interaction much larger than $\lambda_{0}^{2}$ a value determined by considering the assymptotic conditions. 


\section{APPENDIX B}

\section{TRANSITION DENSITIES AND FORM FACTORS}

1. Harmonic Oscillator Wave Functions

Throughout this work, the single particle bound state wave functions used are those for a particle bound in a harmonic oscillator potential. This is a necessity because a complex description of the target nuclei is being attempted. The radial part of these wave functions are given by 60

$$
u_{n \ell}(r)=\pi^{-1 / 4}\left[\frac{2^{n+l+1}(n-1) !}{(2 n+2 \ell-1) ! !}\right]^{1 / 2} \alpha^{\ell+3 / 2} r^{\ell} e^{-\alpha^{2} r^{2} / 2} P_{n l}(r)
$$

where the principle quantum number runs from 1 to $\infty$ and

$$
P_{n \ell}(r)=\sum_{k=0}^{n-1} 2^{k-n+1}(-1)^{k} \frac{(2 n+2 \ell-1) ! !}{(n-k-1) ! k !(2 \ell+2 k+1) ! !}\left(\alpha^{2} r^{2}\right)^{k}
$$

The size parameter, $\alpha$, is given in $F^{-1}$ by

$$
\alpha=\left[\frac{\mathrm{M} \omega}{\hbar}\right]^{1 / 2}=\left[\frac{\mathrm{M} \hbar \omega}{\hbar^{2}}\right]^{1 / 2}=.156(\hbar \omega)^{1 / 2}
$$

where $\hbar \omega$ is the energy separating the major shells of the potential expressed in MeV. Eq. (B.I) and Eq.(B.2) are somewhat more convenient than the more commonly encountered relations which give $u_{n \ell}(r)$ in terms of the associated Laguerre polynomials. The first few $\mathrm{P}_{n \ell}(r)$ are 


$$
\begin{aligned}
& \mathrm{P}_{1 \ell}(r)=1 \\
& \mathrm{P}_{2 \ell}(r)=\frac{2 \ell+3}{2}-\alpha^{2} r^{2}
\end{aligned}
$$

$$
P_{3 \ell}(r)=\frac{1}{2}\left\{\frac{(2 l+3)(2 \ell+5)}{4}-(2 \ell+5) \alpha^{2} r^{2}+\alpha^{4} r^{4}\right\}
$$

From Eq. (2.58), Eq. (2.59), Eq. (2.46"'), and

Eq. (2.47"') it follows that the transition densities can be written

$$
\begin{aligned}
& F^{L S J, T}(r)=\sum_{j j-M} M^{L S J, T}\left(j j^{\prime}\right) u_{n-\ell}-(r) u_{n \ell}(r) \\
& F_{\tau \tau^{\prime}}^{L S J}(r)=\sum_{j j}, M_{\tau \tau}^{L S J}-\left(j j^{\prime}\right) u_{n-\ell}-(r) u_{n \ell}(r)
\end{aligned}
$$

where

$$
\begin{aligned}
& M^{L S J}, T\left(j j^{\prime}\right)=\sqrt{2} \hat{T}<T_{A} T^{T M_{T}}, M_{T_{B}}-M_{T_{A}} \mid T_{B} M_{T_{B}}>\left\langle\frac{1}{2} T \tau_{b}, \tau_{a}-\tau_{b}\right| \frac{1}{2} \tau a^{\prime} \\
& x S\left(J_{A} J_{B} J ; T_{A} T_{B} T ; j j^{\prime}\right) i^{L+\ell-\ell^{\prime}}(4 \pi)^{-I / 2} \sqrt{2} \hat{j} \hat{\ell} \hat{L} \hat{S} \hat{J} \hat{T} \\
& x<L \ell 00 \mid \ell^{\prime} 0>\underline{\bar{X}}\left(j^{\prime} j J ; \ell^{\prime} \ell L ; \frac{1}{2} \frac{1}{2} S\right) \\
& M_{\tau \tau}^{L S J}\left(j j^{\prime}\right)=\sqrt{2} S\left(J_{A}{ }^{J} B^{J} ; j j^{\prime} \tau \tau^{\prime}\right) i^{L+\ell-\ell^{\prime}}(4 \pi)^{-1 / 2} \sqrt{2} \hat{j} \hat{\ell} \hat{L} \hat{S J} \\
& x<L \ell 00 \mid \ell^{\prime} 0>\underline{\bar{X}}\left(j+j J ; \ell / \ell L ; \frac{1}{2} \frac{1}{2} S\right) \text {. }
\end{aligned}
$$

Inspection of the above relations leads to the conclusion that the transition density can always be written in the following form when harmonic oscillator wave functions are used. 


$$
\begin{aligned}
F^{L S J}(r) & =\sum_{N_{a}}^{N_{b}} C_{N}^{L S J} \alpha^{N+3} r^{N} e^{-\alpha^{2} r^{2}} \\
N_{a} & =\left(l+l^{-}\right){ }_{\min } \\
N_{b} & =\left(l+l^{-}+2 n+2 n^{-}-4\right)_{\max }
\end{aligned}
$$

In writing this equation reference to $T$ or $\tau \tau^{\prime}$ has been dropped for convenience. $\mathrm{N}_{\mathrm{a}}$ or $\mathrm{N}_{\mathrm{b}}$ is determined by the contributing. $u_{n \ell} u_{n} \ell^{\prime}$. which yield the minimum or maximum values, respectively, of the bracketed quantities. Note also that the transition density is an even or odd function of $r$ as the parity change in the transition is plus or minus, i.e. only even or odd values of $\mathrm{N}$ are included in summing from $\mathrm{N}$ a to $\mathrm{N}_{\mathrm{b}}$

\section{Macroscopic Vibrational Modei}

Considerable success has attended the use of the macroscopic vibrational model in describing inelastic scattering. There are numerous references to this approach in the literature - Ref. 61 and 62 are but two of these. As there must be a rough correspondence between the microscopic picture and this macroscopic picture it is useful to review this model. A modification of this model is used in the treatment of core polarization which is discussed in Chapter 7 and Appendix D. The following discussion is restricted to even target nuclei which have ground state spin equal to zero. 
In this model the nucleus is likened to a quantized drop of an incompressible, non-viscous fluid. The primary excitations of this system are small surface oscillations (phonons) about spherical equilibrium. The surface of the drop is given by

$$
R(\theta, \phi)=R_{0}\left\{I+\sum_{L M} \alpha_{L M} Y_{L M}^{*}(\theta, \phi)-(4 \pi)^{-I} \sum_{L M}\left|\alpha_{L M}\right|^{2}\right\}
$$

which conserves volume to second order in $\alpha_{L M}$, the deformation parameter. The Hamiltonian for the system is

$$
H=\sum_{L M}\left\{\frac{I}{2 D_{L}}\left|\pi_{L M}\right|^{2}+\frac{1}{2} C_{L}\left|\alpha_{L M}\right|^{2}\right\}
$$

where $D_{L}$ is the mass parameter for excitations of angular momentum $L$ and parity $(-1)^{L}, C_{L}$ is the corresponding stiffness parameter, and $\pi_{L M}$ is the momentum conjugate to $\alpha_{L M}$. In terms of the operators which create and annihilate phonons, $\mathrm{c}_{L M}^{+}$and $\mathrm{c}_{L M}$, the Hamiltonian is written

$$
H=\sum_{L M} \hbar \omega_{L}\left(c_{L M}^{+} c_{L M}+\frac{1}{2}\right)
$$

where $\omega_{L}=\left(C_{L} / D_{L}\right)^{I / 2}$ is the frequency of the phonon designated by $\mathrm{L}$.

The $c_{L M}^{+}$and $c_{L M}$ obey boson commutation relations. If the hydrodynamic description of the system is adhered to strictly, relations for $D_{L}$ and $C_{L}$ are easily obtained. In practice it is necessary to treat them as free parameters. I'he $\pi_{L M}, \alpha_{L M}, c_{L M}^{+}$, and $c_{L M}$ are related as follows 


$$
\begin{aligned}
& 236 \\
& \alpha_{L, M}=[i]\left(\frac{\hbar \omega_{L}}{2 C_{L}}\right)^{1 / 2}\left\{c_{L M}^{+}+(-1)^{L+M} c_{L,-M}\right\} \\
& \pi_{L M}=-i[i]^{-1}\left(\frac{\hbar C_{L}}{2 \omega_{L}}\right){ }^{1 / 2}\left\{c_{L, M}-(-1)^{L+M} c_{L,-M}^{+}\right\} \\
& c_{L M}^{+}=[i]^{-1}\left(\frac{C_{L}}{2 \hbar \omega_{L}}\right)^{I / 2}\left\{\alpha_{L M}-i \frac{\omega_{L}}{C_{L}^{-}} \pi_{L M}^{+}\right\}
\end{aligned}
$$

where [i] is 1 for $L$ even and $i$ for $L$ odd. Equations (B.II) are subject to the conditions that the phonon states transform under rotations and time reversal in the same manner as the single particle wave functions $\phi_{\ell}{ }^{l}(\bar{r})$ which were defined in Chapter 2 and that $R(\theta, \phi)$ has appropriate matrix elements in such a representation. 63 Note that these equations are consistent with the classical reality condition $\alpha_{L M}^{+}=(-1)^{M} x$ $\alpha_{L,-M}$.

It is then assumed that the interaction between a projectile and this liquid drop is only a function of the distance between the projectile and the surface of the drop, i.e. $(r-R)$. Since only small vibrations are being considered it is reasonable to make a Taylor series expansion of the interaction about $R=R_{0}$. To first order in $\alpha_{L M}$ this expansion is

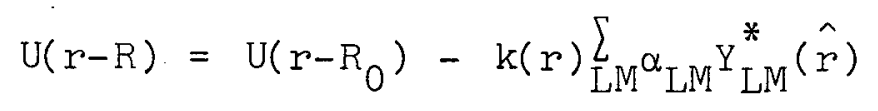

where $k(r)=R_{0} d U\left(r-R_{0}\right) / d r . U\left(r-R_{0}\right)$ is identified as the optical potential which is spherical and describes the elastic scattering. Assuming the usual Woods-Saxon form this potential is written 


$$
\begin{aligned}
U= & -V\left(e^{x}+1\right)^{-1}-i W\left(e^{x}+1\right)^{-1}+4 i W_{D} \frac{d}{d x^{\prime \prime}}\left(e^{x^{\prime \prime}}+1\right)^{-1} \\
& +\left(\hbar / m_{\pi} c\right)^{2} V_{S} r^{-1} \frac{d}{d r}\left(e^{x_{S O}}+1\right)^{-1} \bar{L} \cdot \bar{\sigma}
\end{aligned}
$$

where $x=\left(r-r_{0} A^{1 / 3}\right) / a, x^{\prime}=\left(r-r_{0} A^{1 / 3}\right) / a$, etc. and to which is added the coulomb potential of a uniformly charged sphere of radius $r_{c} A^{1 / 3}$. The potential contains a real volume term, volume and surface imaginary terms, and a real volume spinorbit term. The diffuseness parameters are $a, a^{\prime}$, . . . and the radii are identified as $R_{0}=r_{0} A^{1 / 3}, R_{0}^{-}=r_{0}^{-} A^{1 / 3}$, . Neglecting the Coulomb and spin orbit terms in the potential leads to the following expression for $k(r)$

$\left.k(r)=\left(V_{0} / a\right) \frac{e}{(1+e)^{2}}+W^{\prime} R_{0}^{\prime} / a^{\prime}\right) \frac{e^{\prime}}{\left(1+e^{\prime}\right)^{2}}+4 i\left(w_{D^{\prime}} R_{0}^{\prime} / a^{\prime \prime}\right) \frac{e^{\prime \prime}\left(1-e^{\prime}\right)}{\left(1+e^{\prime \prime}\right)^{j}}(B .14)$ where $e=\exp \left(r-R_{0} / a\right)$,. . Before completing this discussion by defining the form factor for inelastic scattering $F^{S L J}(r)$, it should be noted that the prescription (B.12) for treating the deformation is not the only one which appears in the literature 61,64 , although it is the one used most Irequently. Futher Eq. (B.12) only provides for the treatment of $(L, O, L)$ triads for normal parity transitions. In this model the form factor for the excitation of a single phonon is

$$
\check{F}^{L O L}(r)=-i^{L} \sqrt{2} k(r)\left\langle L|| \alpha_{L}|| O\right\rangle .
$$


Using Eq. (B.Il) gives

$$
\tilde{F}^{L O L}(r)=-i^{L}[i] \sqrt{2} k(r)\left(\frac{\hbar \omega_{L}}{2 C_{L}}\right)^{1 / 2},
$$

thus inelastic scattering experiments provide a measure of the stiffness parameter. It is common practice to tabulate the root mean square deformation in the ground state due to zero point oscillations.

$$
\beta_{L}^{2}=\left\langle\left. 0\left|\sum_{M}\right| \alpha_{L M}\right|^{2} \mid 0\right\rangle=(2 L+1)\left(\frac{\hbar \omega_{L}}{2 C_{L}}\right)
$$

which gives

$$
\tilde{F}^{L O L}(r)=-i^{L}[i] \sqrt{2} k(r) \frac{\beta_{L}}{\hat{L}} .
$$

In this discussion only the matter distribution in the drop has been considered. This fact and the restriction to lowest order is why the description applies only to normal parity transitions. In addition the liquid drop described here can only have excitations of quadrupole order or higher. By introducing other variables, i.e. compressibility, spin, and charge, the model can be generalized to encompass a larger class of vibrations. ${ }^{63,65}$ In Appendix $C$ electromagnetic transitions are considered and the model is extended with the assumption of a uniform distribution of charge throughout the volume of the drop. 
3. Reduced Matrix Elements and Transition Densities for Various Transitions

In order to calculate the transition densities it

is necessary to evaluate the reduced matrix elements of the one body operators which appear in Eq. (2.58') and Eq. (2.59'). In the occupation number representation a one body operator is written

$$
O=\sum_{i} o_{i}=\sum_{\alpha \beta}\left\langle\alpha|0| \beta>a_{\alpha}^{+} a_{\beta}\right.
$$

where $\mathrm{a}^{+}$and a are the fermion creation and annihilation operators which were introduced in Chapter 2. They satisfy anticommutation relations. When using $i-s p i n$ the operator of interest is

$$
0^{L S J}, T=\sum_{i=1}^{N} \frac{\delta\left(r-r_{i}\right)}{r^{2}} T^{L S J}(i) \tau^{T}(i)=\sum_{i=1}^{N} 0^{L S J}, T(i)(B .20)
$$

and when not using $i-s p i n$ it is

$$
O_{\tau \tau^{\prime}}^{L S J}=\sum_{i}^{\prime} \frac{\delta\left(r-r_{i}\right)}{r^{2}} T^{L S S J}(i)=\sum_{i}^{\prime} O^{L S J}(i) .
$$

In the form of Eq. (B.19) these become

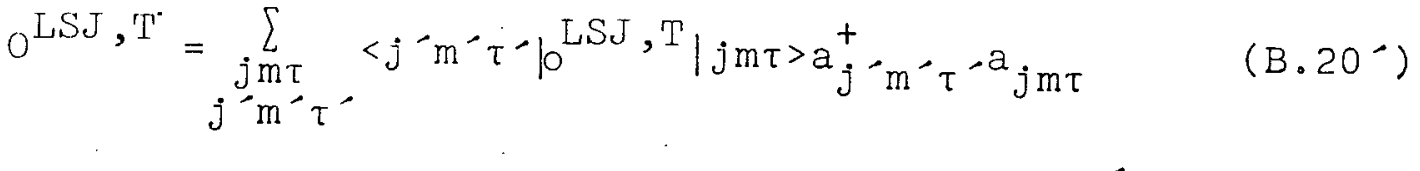

$$
\begin{aligned}
& O_{\tau \tau^{\prime}}^{L S J}=\sum_{j m^{\prime} m^{\prime}}\left\langle j^{\prime} m^{\prime}\left|o^{L S J}\right| j m>a_{j^{\prime} m^{\prime} \tau^{\prime}}^{+} a_{j m \tau}\right.
\end{aligned}
$$


In the discussion which follows $R^{L S J}$ will be used for $\left\langle\mathrm{B}\left\|\mathrm{O}^{\mathrm{LSJ}}\right\| \mathrm{A}\right\rangle$ and a single subscript will be used on $\mathrm{a}^{+}$and a to represent the quantum numbers jmt.

\section{Single Particle Transition}

This is a trivial case and there is no need to introduce i-spin. The initial and final states are $|A\rangle=a_{2}^{+}|C\rangle$ and $|B\rangle=a_{1}^{+} \mid C>$, respectively, where $|C\rangle$ denotes a filled shell state. The following result is easily. obtained.

$$
R_{\tau \tau^{\prime}}^{L S J}=\left\langle j_{1} \| 0^{L S J}|| j_{2}>\delta_{\tau \tau^{\prime}, \tau_{2} \tau_{1}}\right.
$$

The $\delta_{\tau \tau^{\prime}, \tau_{2} \tau_{1}}$ is used with Table 2 of Chapter 2 to determine the force component which is needed. For example, consider. a single neutron transition in the $\left(\mathrm{p}, \mathrm{p}^{\prime}\right)$ reaction. Then $\tau_{1}=\tau_{2}=-\frac{1}{2}$ and the transition goes through the proton-neutron force. For the $(p, n)$ reaction $\tau$ must equal $-\tau^{\prime}=-\frac{1}{2}$ in order for the transition to be allowed; therefore, the single particle must initially be a neutron and a single proton will be left in the final state.

Single Hole Transition

For this case the initial and final states are $|A\rangle=(-1)^{j_{2}-m_{2}} a_{2} \mid C>$ and $\left|B>=(-1)^{j_{1}-m_{1}} a_{1}\right| C>$, respectively. The purpose of the phase was mentioned previously. It follows immediately that 


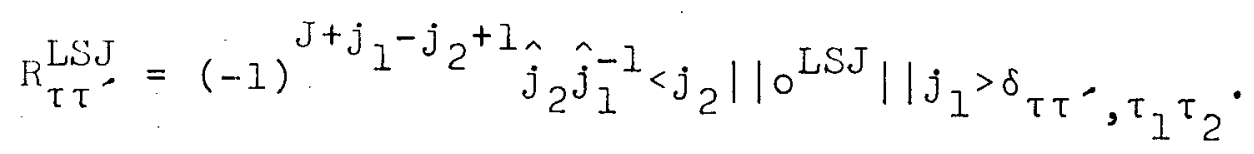

Using the conjugation relation

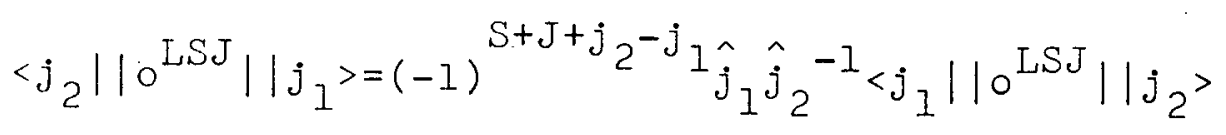

gives for Eq. (B.23)

$$
R_{\tau \tau^{\prime}}^{L S j}=-(-1)^{S}<j_{1}|| 0^{L S J}|| j_{2}>\delta_{\tau \tau^{\prime}, \tau_{1} \tau_{2}} .
$$

'This relation shows that a neutron single hole transition in the $\left(p, p^{-}\right)$reaction is the same as a neutron single particle transition except for the phase factor (-1) ${ }^{S}$ which may have some effect when interferences is important. In the $(p, n)$ reaction the initial state must be a proton hole and the final state is a neutron hole. This indicates the signif'icance of the interchange of $\tau_{1}$ and $\tau_{2}$ in Eq. (B.25) as compared to the ordering in Eq. (B.23).

\section{l'ransitions to Particle-Hole states}

The simplest excitations of closed shell nuclei are particle-hole pairs. In light nuclei with equal neutronproton number i-spin is usually assumed to be a good quantum number and a particle-hole state is written

$$
\begin{aligned}
& |B\rangle=\left|J_{B} M_{B} T_{B} M_{B} T_{B}>=\sum_{p} m_{h} C_{j_{p}}^{J_{B}{ }^{T}{ }_{B}}<j_{p} j_{h} m_{p}-m_{h}\right| J_{B} M_{B}><\frac{l}{2} \cdot \frac{I}{2} \tau_{p}-\tau_{h} \mid T_{B}{ }^{M} T_{B}> \\
& \tau_{p} \tau_{h} \\
& j_{p} j_{h}
\end{aligned}
$$




$$
x(-1)^{j}{ }_{h}^{-m}+1 / 2-\tau h a_{p h}^{+} a_{h} \mid C>
$$

States of this form are obtained by diagonalizing a shell model Hamiltonian in the space of particle-hole pairs. This procedure is referred to as the Tamm-Dancoff Approximation (T.D.A.) and it assumes that the ground state of such a nucleus is a filled shell $|A\rangle=|C\rangle .{ }^{1,2}$ The reduced matrix element describing transitions from the ground state to the states $(B .26)$ is $R^{L S J, T}=\sum_{j_{p} j_{h}} C_{j_{p} j_{h}}^{J_{B} T^{T}} \sqrt{2} \hat{j}_{p}\left[\hat{T}_{B} \hat{J}_{B}\right]^{-1}<j p \frac{1}{2}|| 0^{L S J}, T|| j_{h} \frac{I}{2}>$.

Since the ground state is a filled shell the only allowed values of $\mathrm{JT}$ are $\mathrm{J}_{\mathrm{B}} \mathrm{T}_{\mathrm{B}}$.

For heavier nuclei with unequal neutron-proton number i-spin is usually ignored and particle-hole states of the following form are obtained

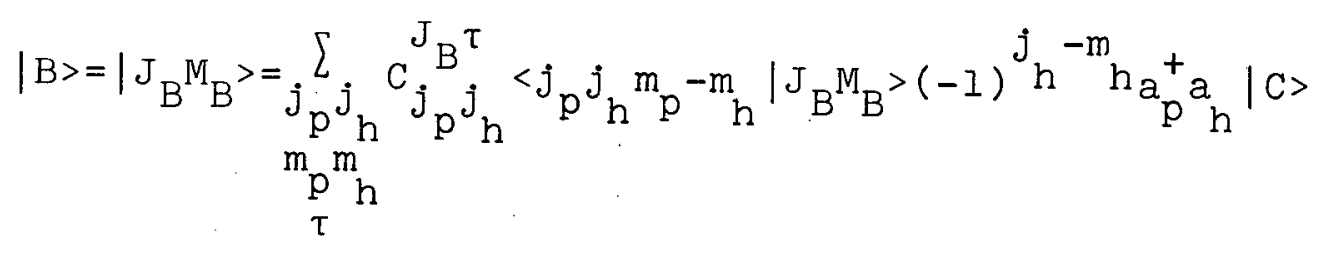

where $\tau$ distinguishes between proton-proton holes and neutron-neutron holes. In this case the reduced matrix elements.

$$
R_{\tau \tau}^{L S J}=\sum_{p} \hat{j}_{h} C_{j_{p} j_{h}}^{J} \hat{j}_{p} \hat{J}_{B}^{-1}<j_{p}|| 0^{L S J} \| j_{h}>
$$


are used. 'l'he subscript on $R$ has $\tau=\tau^{\prime}$ since the initial and f'inal states considered here are states of the same nucleus. Note that the form factor has explicit proton and neutron components when $i-s p i n$ is not used.

\section{Random Phase Approximation Vectors}

The R.P.A. goes a step beyond the T.D.A. in treating closed-shell nuclei. It takes into account in an approximate way that the ground state may have $2 p-2 h, 4 p-4 h$, etc. and that the excited states may have $3 p-3 h, 5 p-5 h$, etc. components in addition to lp-in components. ${ }^{1,2}$ The excitations in the ground state are referred to as gound state correlations (G.S.C.) The inciusion of these higher excitations has an important effect on transition rates as they allow the excited state to be reached by destroying a particlehole pair as well as by creating one.

Disregarding $i-s p i n$ an R.P.A. state vector is given by

$$
\begin{aligned}
& |B\rangle=\left|J_{B} M_{B}\right\rangle=\dot{Q}_{J_{B}{ }_{B} M_{B}}|\tilde{C}\rangle
\end{aligned}
$$

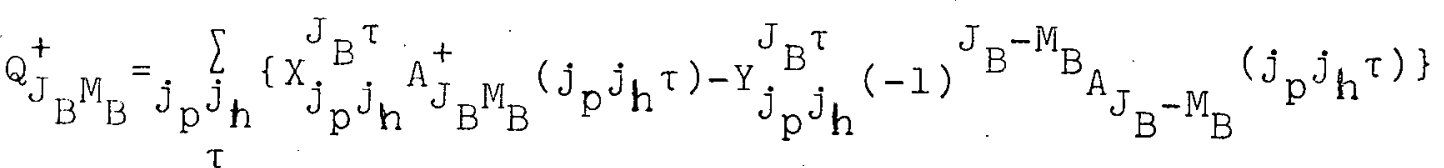

where $|\tilde{C}\rangle$ is the generalized ground state and

$$
A_{J_{B}, M_{B}}^{+}\left(j_{p} j_{h} \tau\right)=\sum_{m_{p} m_{h}}<j_{p} j_{n} m_{p}-m_{h} \mid J_{B} M_{B}>(-1) h_{h}^{j}-m_{h} a_{p}^{+} a_{h} .
$$


I'he second term in Eq. (B.30) represents the G.S.C. The necessary reduced matrix elements are obtained by following the procedure

$\langle\mathrm{B}|\mathrm{O}| \mathrm{A}\rangle=\langle\tilde{\mathrm{C}}|\mathrm{Q} O| \tilde{\mathrm{C}}\rangle=\langle\tilde{\mathrm{C}}|[Q, O]| \tilde{\mathrm{C}}\rangle \tilde{\sim}\langle\mathrm{C}|[Q, O]| \mathrm{C}\rangle$

where the fact $Q \mid \tilde{C}>=0$ is used in introducing the commutator in the third step. It is easy to show that Eq. (B.29) applies with the condition

$$
\mathrm{C}_{j_{p} j_{h}}^{J_{B}^{\tau}}=x_{j_{p} j_{h}}^{J^{\tau}}+(-1)^{S_{Y}}{ }_{j_{p} j_{h}}^{J^{\tau}} \text {. }
$$

Correspondingly for good i-spin Eq. (B.27) prevails with

$$
{ }_{j_{p}{ }^{j} h}^{J_{B} T_{B}}=x_{j_{p}{ }^{j_{h}}}^{J_{B} T_{B}}+(-1)^{S+T_{Y}}{ }_{j_{p}{ }^{j} h}^{\mathrm{T}_{B}} \text {. }
$$

$X$ and $Y$ are generally in phase and they add in non-spin flip amplitudes (for iso-scalar amplitudes if Eq. (B.34) is being considered) and the enhancement due to G.S.C. is. apparent if it is noted that the vectors (B.30) satisfy the normalization condition $\sum\left(X^{2}-Y^{2}\right)=1$ instead of $\sum C^{2}=1$. Like the macroscopic vibrational model, the T.D.A. and R.P.A. are schemes directed towards the explanation of low lying vibrational states in nuclei. The states (B.26), (B.28), and (B.30) may be called phonons.

Transitions Between States of $j^{2}$ Configurations

Forgetting about i-spin the wave function for two nucleons of the same type in the $j^{2}$ configuration is 


$$
|A\rangle=\left|J_{A} M_{A} \tau\right\rangle=\underset{\sqrt{2}}{\frac{l}{2} m_{1} m_{2}}\left\langle j j m_{1} m_{2} \mid J_{A} M_{A}\right\rangle a_{1}^{+} a_{2}^{+}|c\rangle
$$

where $\tau$ again differentiates between protons and neutrons. I'his wave function is normalized and vanishes unless $J_{A}$ is even. For a transition between two states of this type

$$
R_{\tau \tau}^{L S J}=(-1)^{J+1} 2 \hat{j J}_{A}\left\{\begin{array}{lll}
j & j & J \\
J_{B} & J_{A} & j
\end{array}\right\}\left\langle j|| 0^{L S J}|| j\right\rangle
$$

where \{\} is a $6-j$ symbol 58 and $\tau=\tau$ ' because of the restriction to like nucleons. The single particle reduced matrix element vanishes unless $L$ is even and when $J=L$ and $S=1$; therefore transitions starting at the state $J_{A}=0$ do not proceed by spin-flip.

Transitions from $1 p$ to $2 p-$ in states

In treating core polarization as presented in Chapter 7 and Appendix D transitions from a one particle to a two particle-one hole state are encountered. A two particleone hole state is written

$$
\begin{aligned}
& |B\rangle=\left|j_{\perp}\left(j_{p} \bar{j}_{h}\right) J_{c} ; J_{B} M_{B}\right\rangle=\sum_{p} m_{h}\left\langle j_{p} j_{h} m_{p}-m_{h}\left|J_{c} M_{c}><j_{I} J_{c} m_{I} M_{c}\right| J_{B} M_{B}\right\rangle \\
& m_{1} M_{c} \\
& x(-1)^{j}{ }^{j}-m_{h}+a_{1} a_{p}^{+} a_{h} \mid c>
\end{aligned}
$$

where i-spin is not being used. The above wave function is not normalized when $p=I$. This is not important at the present time and will be discussed in Appendix D. The 
reduced matrix element for the transition from a singleparticle state, $|A\rangle=a_{2}^{+}|C\rangle$, to the state (B.37) is

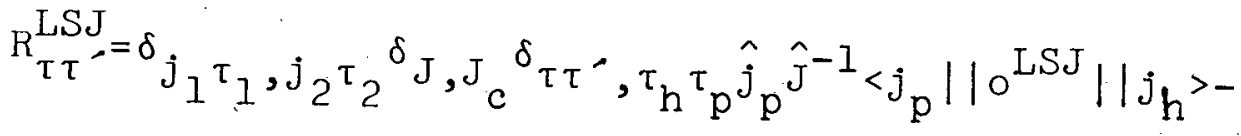

$$
\begin{aligned}
& \left\{\delta_{j_{p} \tau_{p}, j_{2} \tau_{2}{ }^{\delta} \tau \tau^{\prime}, \tau_{h} \tau_{1}}(-1)^{j_{1}+j_{2}+J+J_{c}} \hat{J}_{c} \hat{j}_{1}\left\{\begin{array}{l}
j_{2} j_{h} J \\
j_{l} J_{B} J
\end{array}\right\}\right. \\
& \left.x<j_{1}\left\|o^{L S J}\right\| j_{h}>\cdot\right\}
\end{aligned}
$$

An allowed transition is subject to the condition that $j_{2} \tau_{2}=j_{1} \tau_{1}$ and/or $j_{p} \tau_{p}$ as expected. When $j_{1} \tau_{1}=j_{2} \tau_{2} \neq j_{p} \tau_{p}$ only the first term in Eq. (B. 38) contributes and the reduced matrix element is the same as that for exciting a particlehole pair. This is seen by comparing with Eq. (B.29) and noting that $\tau=\tau^{\prime}$ in Eq. (B:38) when $\tau_{p}=\tau_{h}$ which is the condition for a transition between states in the same nucleus. The second term in Eq. (B.38) differs from the first by recoupling factors which appear simply because the role of the active and spectator particle have been interchanged.

Transitions from $2 p$ to $3 p-1 h$ States

A general expression for a transition from a $2 p$ state to a $3 \mathrm{p}-1 \mathrm{~h}$ state is somewhat cumbersome to write down and tedious to derive. Further fractional percentage must be considered when the three particles are alike and in the same orbit. For the core polarization discussion in Appendix $D$ only a particular result is needed and this is all that 
will be given here. This result is for the case when the $2 p$ state is that of two like nucleons in a $j^{2}$ configuration. Eq. (B.35) gives the wave function for such a state. The $3 p-1 h$ states which are connected to the states $(B .35)$ by a one body operator can only have one particle in an orbit other than $j$. These particular $3 p$-In states can be written

$$
\begin{aligned}
& |B>=|\left[(j j) J_{V},\left(j_{p} \bar{j}_{h}\right) J_{C}\right] J_{B} M_{B}> \\
& =\frac{l}{\sqrt{2}} \sum_{1} m_{2}\left\langle j j m_{l} m_{2}\left|J_{v} M_{v}><j_{p} j_{h} m_{p}-m_{h}\right| J_{c} M_{c}>\left\langle J_{v} J_{c} M_{v} M_{c} \mid J_{B} M_{B}\right\rangle\right. \\
& m_{p} m_{h} \\
& \mathrm{M}_{\mathrm{V}} \mathrm{M}_{\mathrm{C}} \\
& x(-1)^{j} p^{-m_{h}+a_{1}+a_{p}^{+} a_{h} \mid c>}
\end{aligned}
$$

which is normalized as long as $j_{p} \tau_{p} \neq j \tau$ which is to be assumed. I'his state vanishes unless $J_{v}$ is even.

The necessary reduced matrix element is

$R_{\tau \tau}^{L S J J}=\delta_{J_{V}, J_{A}} \delta_{J, J} \delta^{\delta} \tau \tau^{\prime}, \tau_{h} \tau_{p} \hat{j}_{p} \hat{J}^{-1}<j_{p}|| O^{L S J}|| j_{h}>$

where again it is seen that the result is the same as for exciting a particle-hole pair with the 2 p state playing the role of a spectator. Also $\tau=\tau^{\prime}$ when $\tau_{p}=\tau_{h}$ which hols for transition between states of the same nucleus.

4. Note on Phases

In all of the formulas presented in this paper the phases of the bound state wave functions have been fixed by 
demanding that they be invariant under time reversal and rotation of $180^{\circ}$ about the y-axis. ${ }^{63}$ When iso-spin is involved an extended time reversal operation is defined and a rotation of $180^{\circ}$ about the $y$-axis in iso-space must be included. Fixing the phases in this way is one, but not the only way, of guaranteeing the reality of the bound state matrix elements of many operators. This phase convention explains the appearance for the $i^{\ell}$ in the definition of the single particle bound state wave functions given in Chapter 2. Further it plays a role in the conjugation property of a matrix, e.g. Eq. (B.24).

Many workers do not use the $i^{l}$ in their single particle wave functions which is also a satisfactory phase convention. Since the wave functions of various people have been used in obtaining the results of this paper the phase convention of the formulas was not strictly adhered to in the calculations. Of course none of the physical results have been effected. It is generally quite easy to convert from one phase convention to the other. This note serves simply as a reminder that some of the tabulated results which appear will not be consistent with the formulas as far as phases are concerned.

\section{Multipole Coefficients}

For Yukawa interactions and Gaussian interactions closed forms exist for the multipole coefficients. These coefficients are defined in Eq. (2.43) and Eq. (2.44) and appear as $t_{S T L}\left(r_{0} ; r_{I}\right)$ and $t_{S \tau \tau}\left(r_{0} ; r_{I}\right)$ in Eq. (2.46), Eq. (2.47), and later equations. For the Yukawa interaction 


$$
\begin{gathered}
r\left(r_{0 I}\right)=V e^{-m r_{0 I} / m r_{0 I}} \\
f_{L}\left(r_{0} ; r_{I}\right)=4 \pi i V j_{L}\left(i m r_{<}\right) h_{L}^{(+)}\left(i m r_{>}\right)
\end{gathered},
$$

and for the Gaussian interaction

$$
\begin{gathered}
f\left(r_{0 I}\right)=V e^{-m^{2} r_{01}^{2}} \\
f_{L}\left(r_{0} ; r_{I}\right)=4 \pi V i^{L} j_{L}\left(-2 i m^{2} r_{0} r_{I}\right) e^{-m^{2}\left(r_{0}^{2}+r_{I}^{2}\right)}
\end{gathered}
$$

In Eq. (B. 4I) $h_{L}^{(+)}$denotes the spherical Hankel function and $r_{<}$and $r_{>}$denote the lesser and the greater of $r_{0}$ and $r_{1}$. A general force requires that Eq. $(2.44)$ be handled numerically. A reasonably fast routine has been written for the calculation of form factors for the case of an interaction of general radial form. 


\section{APPENDIX C}

\section{INELASTIC ELECTRON-NUCLEUS SCATTERING}

The electromagnetic interaction between an electron and a nucleus can be decomposed into longitudinal Coulomb, transverse electric, and transverse magnetic multipoles. 'The excitation of collective states in normal parity transitions in the $\left(e, e^{\prime}\right)$ reaction proceeds predominately through the Coulomb multipoles. Restricting consideration to these cases the differential cross section, in Born Approximation, is written 55,56

$$
\sigma(\theta)=\sigma_{M}(\theta)|F(q(\theta))|^{2}
$$

where $\sigma_{M}(\theta)$ is the Mott cross section, i.e.

$$
\sigma_{M}(\theta)=4\left(Z e^{2} / \hbar c\right)^{2}\left(k_{f}^{2} / q^{4}(\theta)\right) \cos ^{2}(\theta / 2),
$$

$\theta$ is the scattering angle, $q(\theta)$ is the magnitude of the momentum transfer $\overline{\mathrm{q}}=\overline{\mathrm{k}}_{i}-\overline{\mathrm{k}}_{f}$, and $\overline{\mathrm{k}}_{i}$ and $\overline{\mathrm{k}}_{f}$ are the initial and final momentum of the electron. The Mott cross section describes the elastic scattering of a high energy electron by a point charge. Most of the kinematics is contained in this term. $F(q(\theta))$ is the inelastic electron scattering form factor which contains all of the nuclear structure information. It is defined by 


$$
\left|F^{\prime}(q)\right|^{2}=\hat{J}_{A}^{-2} \sum_{B} \mathbb{M}_{A}\left|\frac{1}{Z e} \int e^{i \bar{q} \cdot \bar{r}_{r}}<B\right| \rho(\bar{r})\left|A>d^{3} r\right|^{2} .
$$

Eq. (C.3) contains a nuclear matrix element of the charge density operator which is written as follows

$$
\begin{aligned}
& \rho(\bar{r})=\frac{e}{2} \sum_{i=1}^{N} T_{0}^{T}(i) \delta\left(\bar{r}-\bar{r}_{i}\right) \\
& \rho(\bar{r})=e_{i}^{\sum_{i}^{\prime} \delta\left(\bar{r}-\bar{r}_{i}\right)}
\end{aligned}
$$

when i-spin is or is not used, respectively. The sum on $i$ in Eq. (C.5) runs only over target protons while $\frac{1}{2} \sum_{T} \tau_{0}^{T}$ serves as a proton counter when i-spin is used. A little algebra leads to the following expressions for Eq. (C.3) for the cases defined in Eq. (C.4) and Eq. (C.5).

$$
\begin{aligned}
|F(q)|^{2}= & \frac{2 J_{B}+I}{2 J_{A}+I} \sum_{L}\left|(\pi / 2)^{I / 2} z^{-1} f_{L^{\prime}}^{\infty}(q r) \partial^{L O L}(r) r^{2} d r\right|^{2} \\
& \partial^{L O L}(r)=\sum_{T} F_{p p}^{L O L}, T(r) \\
\left|F^{\prime}(q)\right|^{2}= & \frac{2 J_{B}+1}{2 J_{A}^{+I}} \sum_{L}\left|(2 \pi)^{I / 2} Z^{-1} \int_{0}^{\infty} j_{L}(q r) F_{p}^{L O L}(r) r^{2} d r\right|^{2}
\end{aligned}
$$

In these equations $\mathrm{F}_{\mathrm{pp}}^{\mathrm{LOL}, \mathrm{T}}(r)$ and $\mathrm{F}_{\mathrm{p}}^{\mathrm{LOL}}(r)$ are the transition densities defined in Eq. (2.58') and Eq. (2.59'), respectively. The transition density defined in Eq. $\left(2.58^{\prime}\right)$ is reaction dependent because of the Clebsch-Gordan coefficient which contains the $i$-spin projection quantum numbers of the 
projectile. The subscript pp on $\mathrm{F}^{\mathrm{LOL}, \mathrm{T}}(r)$ in $\mathrm{Eq} \cdot\left(\mathrm{C} .4^{\circ}\right.$ ) serves to specify the transition density for the $\left(p, p^{\prime}\right)$ reaction, i.e. $\tau_{a}=\tau_{b}=\frac{I}{2}$. In Eq. $\left(C .5^{\prime}\right)$ the subscript $p$ on $\mathrm{F}^{\mathrm{LOL}}(\mathrm{r})$ defines the proton transition density, $\tau=\tau=\frac{1}{2}$. It should be pointed out that for the transitions under consideration only the lowest allowed L-transfer will be important. For transitions where more than one L-transfer is likely to be important the treatment will usually have to include the transverse multipoles as well as the longitudinal ones. In such cases the relationship between the $\left(e, e^{\prime}\right)$ and $\left(p, p^{\prime}\right)$ reaction is not as direct as that seen by comparing Eq. (C. $4^{\prime}$ ) and Eq. (C.5') with Eq. (2.58") and Eq. (2.59"). For this case the inelastic electron scattering form factor is related to the Bessel transform of the proton transition density while the inelastic nucleon scattering form factor is obtained by transforming the proton and neutron transition densities with the appropriate multipole coefficient of the two-body interaction.

In practice it is necessary to include two corrections in Eq. (C. $\left.4^{\prime}\right)$, and Eq. (C. $\left.5^{\prime}\right)$. This is accomplished by multiplying these relations by $f^{2}(q)$ where 55

$$
f(q)=\exp \left[-q^{2}\left(a_{p}^{2}-1 / \alpha^{2} A\right) / 4\right]
$$

This serves to correct for the finite size of the proton (first term) and for center of mass motion (second term) 
which is necessary because the shell model wave functions are referred to the center of the oscillator well. The parameter $a_{p}^{2}$ fixes the size of the proton distribution which has been taken to be Gaussian, $\alpha$ is the harmonic oscillator constant, and $A$ is the target mass. In the calculations of this work $a_{p}^{2}=.43 F^{2}$ is used. In principle center of mass corrections should be included in the $\left(\mathrm{p}, \mathrm{p}^{\prime}\right)$ calculations also. This is difficult because the D.W.A. is being used and this smali correction is ignored as a matter of convenience.

A closed expression for the inelastic electron scattering form factor can be obtained when harmonic oscillator wave functions are used by inserting Eq. (B.7) into Eq. (C. $\left.4^{\prime}\right)$ or Eq. (C.5') and using the following Integration formula

$$
\begin{gathered}
\int^{\infty} e^{-\alpha^{2} r^{2}} \cdot J_{v}(q r) r^{\mu-1} d r=\Gamma\left[\frac{1}{2}(\mu+\nu)\right](q / 2 \alpha)^{\nu}\left[2 \alpha^{\mu} \Gamma(1+\nu)\right]^{-1} e^{-q^{2} / 4 \alpha^{2}} \\
\quad x F^{2}\left(\frac{1}{2}(\nu-\mu)+1|\nu+1| q^{2} / 4 \alpha^{2}\right)
\end{gathered}
$$

where $\Gamma()$ denotes the $\Gamma$-function, $F(||)$ is the confluent hypergeometric function, and $J_{v}$ is the ordinary Bessel function. The confluent hypergeometric function is defined by

$$
\begin{aligned}
& F(\lambda|\rho| z)=\sum_{n=0}^{\infty} \frac{(\lambda)_{n} z^{n}}{(\rho)_{n} n !} \\
& \lambda_{0}=1 ; \lambda_{n}=\lambda(\lambda+1) \ldots(\lambda+n-1) \quad n \geq 1 \\
& \rho_{0}=1 ; \rho_{n}=0(\rho+1) \ldots(\rho+n-1) \quad n \geq 1
\end{aligned}
$$


and is a finite polynomial when $\lambda$ is an integer less than or equal to zero. The spherical Bessel function and orginary Bessel function are related by

$$
j_{L}(q r)=\sqrt{ } \frac{\pi}{2 q r} J_{L+1 / 2}(q r) .
$$

For a definite value of $L$ it can be shown that

$$
\begin{aligned}
|F(q)|^{2}= & n \frac{2 J_{B}+1}{2 J} \frac{\pi^{2}}{2 z^{2}}\left(\frac{q}{\alpha}\right)^{2 L} \times[(2 L+1) ! !]^{-2} f^{2}(q) \exp \left(-q^{2} / 2 \alpha^{2}\right) \\
& \times\left\{\sum_{N}^{N} C_{a} C_{N}^{L O L}(L+N+1) ! ! 2^{-(L+N+2) / 2} F\left(\frac{1}{2}(L-N)|L+3 / 2| q^{2} / 4 \alpha^{2}\right)\right\}^{2}
\end{aligned}
$$

where $n=1 / 4$ when $i-s p i n$ is being used and $n=1$ when it is not used. The correction factor $f^{2}(q)$ is defined in Eq. (c.6).

The macroscopic vibrational model might also be applied to inelastic electron scattering. The treatment is the same as that for inelastic nucleon scattering which was outlined in Section 2 of Appendix B, but deformation of the charge density is considered in place of the deformation of the potential for nucleon scattering. The charge density expanded to first order in the $\alpha_{L M}$ is

$$
\rho(r-R)=\rho\left(r-R_{0}\right)-R_{0} \frac{d \rho\left(r-R_{0}\right)}{d r} \sum_{L M^{\alpha} L M^{\alpha} Y_{L M}^{*}}
$$

where $\rho\left(r-R_{0}\right)$ is the spherical ground state charge distribution. Assuming a Woods-Saxon form 


$$
\rho\left(r-R_{0}\right)=\rho_{0}\left[I+\exp \left(\frac{r-R_{0}}{a}\right)\right]^{-1}
$$

and $\rho_{0}$ is fixed by the condition $\int \rho d^{3} r=z e$. In this model the inelastic electron scattering form factor for the excitation of a single phonon of order $L$ is

$$
\begin{gathered}
|F(q)|^{2}=\frac{4 \pi \beta_{L}^{2}}{(Z e)^{2}}\left|f^{\infty} j_{L}(q r) h(r) r^{2} d r\right|^{2} \\
h(r)=R_{0} \frac{d \rho\left(r-R_{0}\right)}{d r}
\end{gathered}
$$

A normal parity $\gamma$-transition involving a collective state will proceed predominately through a single transverse electric multipole. The long wave-length approxiration is valid for $;$-transitions and in this particular instance the inverse electromagnetic lifetime is given by 56

$$
\begin{array}{r}
\omega_{\gamma}=8 \pi c_{\frac{e^{2}}{K C}} \frac{(L+1)}{L[(2 L+1) ! !]^{2}} k^{2 L+I_{B}(E L)} \\
B(E L)=\sum_{M M_{B}}\left|\frac{I}{e} \int r^{L_{Y}}{ }_{L M}(\hat{r})<B\right| \rho(\bar{r})\left|A>d^{3} r\right|^{2}
\end{array}
$$

where $L$ gives the multipolarity of the radiation, $k$ is its wavenumber, and $B(E L)$ is the reduced transition probability. Note that the latter quantity is directional in that

$$
B\left(E L ; J_{A} \rightarrow J_{B}\right)=\frac{2 J_{B}+1}{2 J_{A}+1} B\left(E L ; J_{B} \rightarrow J_{A}\right) \text {. }
$$


From Eq. (C.4) and Eq. (C.5) it follows that

$$
\begin{aligned}
& B(E L)=\frac{2 J_{B}+1}{2 J_{A}+1} \frac{1}{8}\left|f_{0}^{\infty} r^{L+2}{ }_{F}^{L O L}(r) d r\right|^{2} \\
& B(E L)=\frac{2 J_{B}+1}{2 J_{A}+1} \frac{1}{2}\left|\int_{0}^{\infty}{ }_{0}{ }^{L+2} F_{p}^{L O L}(r) d r\right|^{2}
\end{aligned}
$$

for the case when $i-s p i n$ is and is not used. For the excitation of a single phonon in the macroscopic vibrational picture it follows that

$$
B(E L ; 0+L)=\left(\frac{\beta}{e}\right)^{2}\left|\int_{0}^{\infty} r^{L+2} h(r) d r\right|^{2}
$$

which reduces to

$$
B(E L ; 0 \rightarrow L)=\left(\frac{3 Z}{4 \pi} R_{0}^{L} B_{L}\right)^{2}
$$

for the uniform charge distribution. These relations show that electric $\gamma$-transitions provide information about the proton transition density, however, this information is not as valuable as that obtained in inelastic electron scattering experiments since the integrals in Eq. (C.16) Eq. (C.17) are most sensitive to the tail of the density whereas the Bessel transform of the transition density samples different regions of the density as $q$ is varied. 
APPENDIX D

CORE POLARIZATION

\section{Introduction}

In the opening paragraphs of Chapter 7 , several approaches were mentioned for estimating the effect of core polarization on the properties of the low lying states of nuclei with a few nucleons outside of a closed sheil. 'There is one essential point in all of these methods-the basic configurations needed to describe the low lying states of these nuclei are not those of the simple shell model $\left|A_{n}\right\rangle$, which consist of valence nucleons distributed about a filled shell, but the configurations given by first order perturbation theory

$$
\left|\tilde{A}_{n}\right\rangle=\left|A_{n}>+\sum_{n}\left(E_{A_{n}}-E_{C_{n}}\right)^{-1}<C_{n}\right| V^{-}\left|A_{n}>\right| C_{n}>
$$

which contain admixtures of core excited states, $\left|\mathrm{C}_{n}\right\rangle$. In Eq. (D.1) $\mathrm{E}_{A_{\mathrm{n}}}$ and $\mathrm{E}_{\mathrm{C}_{\mathrm{n}}}$ are the unperturbed energies of the states $\left|A_{n}\right\rangle$ and $\left|C_{n}\right\rangle$, respectively, and $V^{\prime}$ is the interaction coupling the valence nucleons to the core. In general, when there are more than one valence nucleons, the complete wave function for a particular state 
in the nucleus is given by a linear combination of the configurations (D.I), i.e.

$$
|A\rangle=\sum_{n=1}^{N} A_{n}^{A}\left|\tilde{A}_{n}\right\rangle
$$

where the $A_{n}^{A}$ are obtained by diagonalizing an effective Hamiltonian for the nucleus in the basis $\left\{\mid A_{n}>; n=1, N\right\}$. Matrix elements of the effective two-body interaction between valence nucleons are defined by

$$
<A_{n}\left|\gamma_{\text {eff }}\right| A_{n},>=<A_{n}|V| A_{n},>+\sum_{C_{n}} \bar{E}_{C_{n}}^{-1}<A_{n}\left|V^{-}\right| C_{n}><C_{n}\left|V^{\prime}\right| A_{n}->(D .3)
$$

The first term on the right in Eq. (D.3) is the usual shell model matrix element, where $V$ is the two-body force between valence nucleons, and the second term contains the effect of the coupling of the valence nucleons to the core. The latter term is similar, but not equivalent, to the energy correction dictated by second order perturbation theory. $\bar{E}_{C_{n}}$ is an energy characteristic of the core excitation in the state $\left|C_{n}\right\rangle$. It can only be approximately fixed in a state independent manner.

No attempt has been made at being complete in writing down these formulas as they are discussed in detail in the references cited in Chapter 7. In the language of Kuo and Brown, $\mathcal{V}_{\text {eff }}$ is the renormalized G-matrix and there is no distinction between $V$ and $V^{\prime}$ which is identified as the "bare" G-matrix. In applying Eq. (D.3) to systems with two valence nucleons Kuo and Brown use an average energy 
denominator for $\overline{\mathrm{E}}_{\mathrm{C}_{\mathrm{n}}}=\overline{\mathrm{E}}$ where $\overline{\mathrm{E}}=-2 \hbar \omega$ for positive parity states and -hw for negative parity states.

Eq. (D.I) and Eq. (D.3) can be written somewhat more compactly as

$$
\begin{gathered}
\left|\tilde{A}_{n}\right\rangle=\left[I+\left(E_{A_{n}}-H_{0}\right)^{-1} P V^{-} \cdot\right]\left|A_{n}\right\rangle \\
<A_{n}\left|\nu_{\text {eff }}\right| A_{n}>=\left\langle A_{n}\left|\left\{V+V-\frac{P}{E} V^{\prime}\right\}\right| A_{n}->\right.
\end{gathered}
$$

where $\mathrm{H}_{0}$ is the unperturbed Hamiltonian and

$$
P=\sum_{C_{n}}\left|C_{n}><C_{n}\right|
$$

Matrix elements of one body operators between states of the form (D. '') are given by

$\left.\left\langle\tilde{B}_{n}|T| \tilde{A}_{n}\right\rangle=<B_{n}\left|\left\{T+T\left(E_{A_{n}}-H_{0}\right)^{-I} P V^{\prime}+V^{\prime} P\left(E_{B_{n}}-H_{0}\right)^{-I_{T}}\right\}\right| A_{n}\right\rangle \cdot \quad(D .5)^{\circ}$

The first term in Eq. (D.5) is simply the direct action of $\mathrm{T}$ on the valence nucleons while the last two terms account for the possibility of the transition being affected through the intermediary of the core. This is analagous to Eq. (D.3) where the shell model matrix element contains the effect of the valence nucleons interacting through thejr mutual force and the second term allows them to interact through the core.

'The necessary formulae for the specific models used in this, work will now be developed. The macroscopic treat- 
ment of core polarization will be considered first as the basic results are displayed in a somewhat more revealing form than they are in the microscopic treatment.

2. Macroscopic Treatment of Core Polarization

When the macroscopic vibrational model is used to describe the core $^{\prime}$ is given by

$$
\left.V^{\prime}=-\sum_{i} k_{V}\left(r_{i}\right) \sum_{L M^{\alpha}}^{\alpha} M_{L M}^{*} \hat{r}_{i}\right)
$$

where the sum on $i$ runs over the valence nucleons, $k_{v}\left(r_{i}\right)=$ $-R_{0} d^{-}\left(r_{i}-R_{0}\right) / d r_{i}$, and $U^{-}\left(r_{i}-R_{0}\right)$ is the shell model potential seen by the ith valence nucleon. The non-spherical component of the interaction of a projectile with such a system, Eq. (B.12), has the same form except the optical potential appears in place of the shell model potential. The one body operator appearing in Eq. (D.5) has two components,

$$
\mathrm{T}=\mathrm{T}_{\mathrm{V}}+\mathrm{T}_{\mathrm{C}}
$$

where $\mathrm{T}_{\mathrm{V}}$ is the valence component and $\mathrm{T}_{\mathrm{C}}$ is the core component. For inelastic proton-nucleus scattering $\mathrm{T}_{\mathrm{V}}$ is the force between the valence nucleons and the proton projectiles and $T_{C}$ is the second term in Eq. (B.12). Isospin is not considered in the treatment and the force in $\mathrm{T}_{\mathrm{V}}$ is taken to include the exchange interaction. For normal parity electromagnetic transitions $\mathrm{T}_{\mathrm{V}}$ is the density operator of Eq. (C.5) with the sum on $i$ running over valence protons and $T_{C}$ is the second term in Eq. (C.II). 
Introducing explicit reference to the core, the states $\left|A_{n}\right\rangle$ and $\left|B_{n}\right\rangle$ in Eq. (D.5) are written $\left|A_{n} O\right\rangle$ and $\left|\mathrm{B}_{\mathrm{n}} \mathrm{O}\right\rangle$, respectively, and the projection operator is $P=\sum_{J M_{J}}\left|C_{n} L ; J M_{J}><C_{n} L ; J M_{J}\right|=\underset{C_{n}}{\sum_{L M}}\left|C_{n} L M><C_{n} L M\right|$

where $C_{n}$ now refers only to the valence configuration and LM designates a one phonon state. The first form of $P$ on the right in Eq. (D.8) contains states with good total angular momentum while in the second form the uncoupled representation is used. Also note that $\mathrm{H}_{0}$ takes the form

$$
\mathrm{H}_{0}=\sum_{i} U^{\prime}\left(r_{i}-R_{0}\right)+H_{C}
$$

where $\mathrm{H}_{\mathrm{c}}$, the core Hamiltonian, is defined in Eq. (B.10). The probability amplitude for the component $\mid C_{n} L ; J M_{J}>$ in the wave function $\left(D . I^{\prime}\right)$ is $\left(E_{A_{n}}-E_{C_{n}} \nVdash \omega_{I}\right)^{-I}$ times $<C_{n} L ; J\left|V^{-}\right| A_{n}>=\delta J_{A_{n}}[i](-1)^{J_{C_{n}}{ }^{-J} A_{n}} \frac{\hat{J}_{n}}{\hat{J}_{A_{n}}}\left(\frac{\hbar \omega_{L}}{2 C_{L}}\right)^{I / 2}<k v_{v}>$

$$
x<C_{n}|| Y_{L}|| A_{n}>
$$

A little algebra gives the following result for Eq. (D.5)

$$
\begin{aligned}
\left\langle\tilde{B}_{n}|T| \tilde{A}_{n}>=\right. & <B_{n}\left|T_{V}\right| A_{n}>+\sum_{L M}\left\{2 \hbar \omega_{L}\left[Q^{2}-\left(\hbar \omega_{L}\right)^{2}\right]^{-1}\left(\hbar \omega_{L} / 2 C_{L}\right)\right. \\
& \left.\left.x f(r)\left[i^{-L_{Y}}{ }_{L H}^{*}(\hat{r})\right]<B_{n}\left|\sum_{i} k_{v}\left(r_{i}\right) i^{L_{Y} Y_{L}}\left(\hat{r}_{i}\right)\right| A_{n}>\right\} \quad(D .] .0\right)
\end{aligned}
$$


where $Q=E_{A_{n}}-E_{B_{n}}$ and $f(r)$ is either $k(r)$ (Eq. B. 12) or $h(r)(E q . C . l l)$. This is the same as the result of Love and Satchler. ${ }^{15}$ Further it is easy to show that the second term on the right in Eq. (D3) becomes

$$
\begin{aligned}
<A_{n}\left|V^{\prime} \frac{P}{\bar{E}} V^{\prime}\right| A_{n}>= & \sum_{L M^{E}} \bar{E}^{-1}\left(K \omega_{L} / 2 C_{L}\right)<A_{n}\left|\sum_{i} k_{V}\left(r_{i}\right) i I_{L M}\left(\hat{r}_{i}\right)\right| C_{n}> \\
& x<C_{n}\left|\sum_{i} k_{V}\left(r_{i}\right) i-L_{Y_{L M}^{*}}^{*}\left(\hat{r}_{i}\right)\right| A_{n}>
\end{aligned}
$$

where it must be remembers that the states $\left|C_{n}\right\rangle$ are simply shell model states. Using closure Eq. (D.II) becomes $<A_{n}\left|V-\frac{P}{\vec{E}} V-\right| A_{n}->=$

$\sum_{L M} \bar{E}^{-1}\left(x \omega_{L} / 2 C_{L}\right)<\left.A_{n}\right|_{i \neq j} k_{V}\left(r_{i}\right) k_{V}\left(r_{j}\right) Y_{L M}\left(\hat{r}_{i}\right) Y_{L M}^{*}\left(\hat{r}_{j}\right) \mid A_{n} \rightarrow$

where the self energy terms $i=j$ have been excluded as their effect is assumed to be incorporated into the shell model potential.

Eq. (D.10) and Eq. (D.I2) are the essential relations for the macroscopic treatment of core polarization. Note that the collective model Hamiltonian has only one single phonon state for each value of LM. As was pointed out in Section 1 of Chapter 7, this does not have any physical significance with respect to the actual core nucleus. The model is used here as a vehicle for parameterizing the core polarization effects. In some calculations $\not{ }^{\prime} \omega_{L}$ and $\mathrm{C}_{\mathrm{L}}$ will characterize a physical core state and in others 
they define an "effective" core phonon. In discussing the macroscopic vibrational model in Appendix $B$ it was pointed out that only vibrations of quadrupole order or higher fell within the framework of the model. This restriction is ignored here with the note that generalizations required to bring in other vibrations may not preserve the form of $\mathrm{k}_{\mathrm{v}}, \mathrm{k}$, and $\mathrm{h}^{65}$ which have been given previously.

The inelastic proton-nucleus scattering form factor corresponding to the transition matrix element (D.10) is

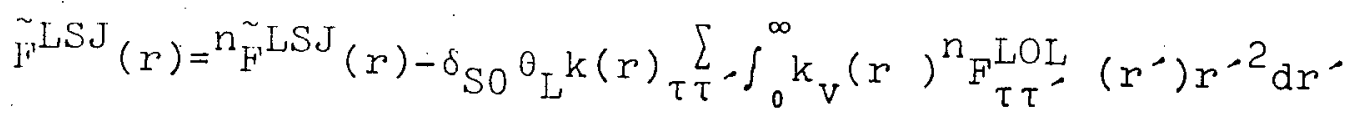

$$
\begin{aligned}
& \theta_{L}=-\frac{\left(\hbar \omega_{L}\right)^{2}}{Q^{2}-\left(\hbar \omega_{L}\right)^{2}} \frac{I}{C_{I}} \approx \frac{I}{C_{L}} \quad\left(\hbar \omega_{L}\right)^{2}>>Q^{2}
\end{aligned}
$$

where the superscript $n$ indicates that only a transition between basic shell model configurations is being considered. The sum on $\tau{ }^{\prime}$ ' is necessary in general since the form factor may have neutron and proton components even when the initiai and final states are simple shell model conligurations. For example, think of a transition between states formed from a proton and a neutron in the same orbital. The fact that the subscript $\tau \tau^{\prime}$ does not appear on $\mathrm{k}_{\mathrm{v}}\left(\mathrm{r}^{\prime}\right)$ amounts to neglecting any differences between neutron and proton wells for the same orbital. From Eq. (B.6) it follows that Eq. (D.13) can be written

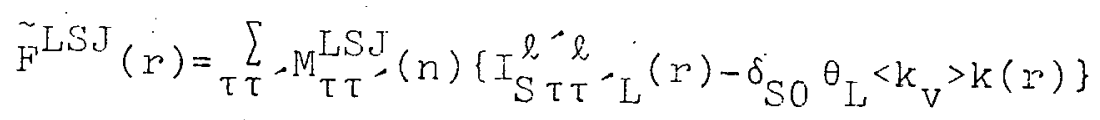


where

$$
I_{S T \tau^{\prime} L^{\prime}}^{\ell^{\prime}}(r)=\int_{0}^{\infty} \nu_{S T \tau^{\prime} L}\left(r ; r^{\prime}\right) u_{n^{\prime} \ell^{-}}\left(r^{\prime}\right) u_{n \ell}\left(r^{\prime}\right) r^{-2} d r^{\prime}
$$

as in $\mathrm{Eq} .\left(2.47^{\circ}\right)$ and

$$
<k_{v}>=\int_{0}^{\infty} k_{v}\left(r^{\prime}\right) u_{n} \ell^{-}\left(r^{\prime}\right) u_{n \ell}\left(r^{\prime}\right) r^{-2} d r^{\prime}
$$

In Eq. (D.14) the contribution due to core polarization appears as a modification of the radial form factor. This modification is scaled by the factor $\theta_{\mathrm{L}}<\mathrm{k} v$ which has $\mathrm{sign}$ opposite to that of $I_{S \tau \tau}^{\ell^{\prime} \ell}{ }^{\prime} L^{-}(r)$ at large $r$. Since $k(r)$ is positive it leads to enhancement of the transition. Further in this model the modification only appears in $S=0$ amplitudes. Deforming the spin-orbit term in the optical potential would bring in the possibility of core polarization contributions in "spin-flip"amplitudes. Note that the form factor is proportional to $\mathrm{M}_{\tau \tau}^{\mathrm{LSJ}}(\mathrm{n})$ which is the geometrical factor characteristic of the transition from the shell model state $\left|A_{n}\right\rangle$ to the state $\left|B_{n}\right\rangle$. As the selection rules for the transition are contained in this factor, it is clear that they have not been effected by these considerations.

For normal parity electromagnetic transitions, inelastic electron-nucleus scattering, or $\gamma$-transitions, $\mathrm{F}_{\mathrm{p}}^{\mathrm{LOL}}(r)$ in $\mathrm{Eq} \cdot\left(\mathrm{C} .5^{\prime}\right)$ or $\mathrm{Eq} \cdot(\mathrm{C} .17)$ becomes

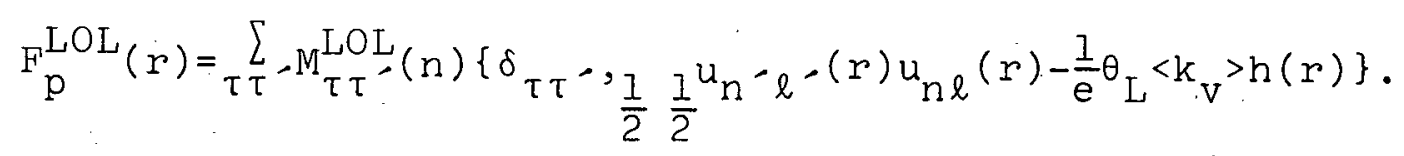


For the $\left(e, e^{\prime}\right)$ reaction the correction factor (c.6) would only be applied to the first term on the right in Eq. (D.17). For a uniform charge distribution the expression for $B(E L)$, Eq. (C.i7), is

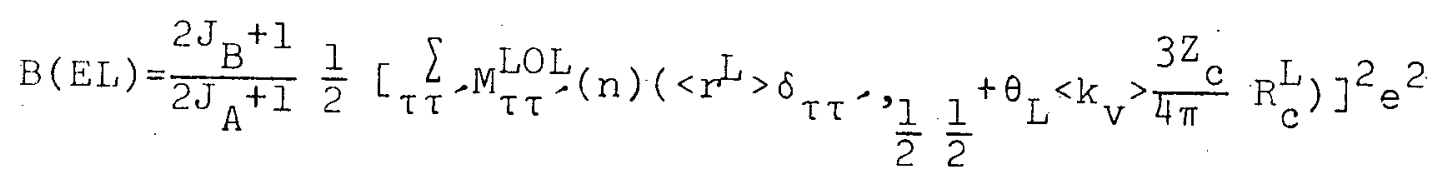

where the subscript $c$ denotes core and

$$
\left\langle r^{L}>=\int_{0}^{\infty} r^{L} u_{n-\ell}-(r) u_{n \ell}(r) r^{2} d r\right.
$$

These relations are completely analagous to those for the $\left(p, p^{\prime}\right)$ reaction and it is seen that there is a core contribution even when the valence nucleons are neutrons. The transitions are enhanced as $\left.\theta_{L}<k_{v}\right\rangle$ has the same sign as $\left\langle r_{L}\right\rangle$ or $u_{n} \cdot \ell^{-}(r)$ $u_{n \ell}(r)$ at large $r$ and $h(r)$ is negative.

Results D.(14) and D.(17) can be obtained by restricting consideration entirely to the valence configurations and assuming the interaction between the proton projectile and the ith valence nucleon to have the form

$$
V\left(\bar{r}-\bar{r}_{i}\right)=V\left(\bar{r}-\bar{r}_{i}\right)-K(r) k_{V}\left(r_{i}\right) \sum_{L M}{ }^{\theta} Y_{L M}^{*}(\hat{r}) Y_{L M}\left(\hat{r}_{i}\right)
$$

or that the density operator for the ith valence nucleon is $\rho(\bar{r})=e_{i}^{*} \delta\left(\bar{r}-\bar{r}_{i}\right)-h(r) k_{V}\left(r_{i}\right) \sum_{L M} \theta_{I} Y_{L M}^{*}(r) Y_{L M}\left(\hat{r}_{i}\right)$

with $e_{i}=0$ or $I$ as the $i$ th valence nucleon is a neutron or a protion, respectively, lupher $\mathrm{Eo}$. (1,18) can be written 


$$
B(E L)=\frac{2 J_{B}+I}{2 J+I} \frac{1}{2}\left[\sum_{\tau} \tau^{-}, M_{\tau \tau}^{L O L}(n)<r^{L}>e_{\tau \tau}-\right]^{2}
$$

where $e_{\tau} \tau$, is the effective charge defined by

$$
e_{\tau \tau^{\prime}=e \delta}=e \tau^{\prime}, \frac{1}{2} \frac{1}{2}+\frac{3}{4 \pi} z_{c} \in R_{c}^{L} \frac{\left\langle k_{v}>\right.}{\left\langle r^{L}>\right.} \theta_{L} .
$$

The above relations clearly display the renormalization of transition operators due to core polarization. The renormalization is dependent on the valence configuration. This dependence appears in $k_{v}$ or $\left\langle k_{v}\right\rangle,\left\langle r^{L}\right\rangle$, and in the $\theta_{L}$. From Eq. (D.3) and Eq. (D.I2) it also follows that the renormalized force between the $i$ th and $j$ th valence nucleons is

$$
\gamma_{\text {eff }}\left(\bar{r}_{i}-\bar{r}_{j}\right)=V\left(\bar{r}_{i}-\bar{r}_{j}\right)+k_{v}\left(r_{i}\right) k_{v}\left(r_{j}\right) \sum_{L M} \bar{E}^{-1}\left(h \omega_{L} / C_{L}\right) Y_{L M}^{*}\left(\hat{r}_{i}\right) Y_{L M}\left(\hat{r}_{j}\right)
$$

which has the same form as Eq. (D.20) which gives the force between an unbound proton and a valence nucleon.

So far the question of configuration mixing has been ignored. This effect is contained in Eq. (D.2) and can be included by multiplying the right hand side of Eq. (D.14) and Eq. (D.17) by $A_{n_{1}}^{A} A_{n}^{B}$ and summing over $n$. There is some difficulty in using this approach when there is a great deal of configuration mixing as ambiguities may result in specifying the state dependent parameters which were mentioned above. 
When considering the coupling of the valence nucleons to a physical state of the core the $\theta_{L}$ is well defined as $C_{L}$ and $\hbar \omega_{L}$ can be determined from the transition in the core nucleus which starts at the ground state and ends at the state in question. The $\left(p, p^{\prime}\right)$ reaction, the (e, $\left.e^{\prime}\right)$ reaction, or $\gamma$-transitions can be used for this determination. Love and Satchler 15,16 assume $\theta_{L}$ characterizes an effective core phonon with $h \omega_{L}>>Q$ so that $\theta_{L}=I / C_{L}$. They consider transitions in the $\left(p, p^{\prime}\right)$ reaction and $\theta_{L}$ is fixed from an analysis of corresponding $\gamma$-transitions. Another method which is used in this work is to determine the $\theta_{L}$ from the spectrum.

For example, corsider a nucleus with two like valence rucleons and assume that these nucleons are restricted to the $(j)^{2}$ configuration. The low lying states of this nucleus will have $\mathrm{J} 0^{+}, 2^{+}, \ldots(2 \mathrm{j}-1)^{+}$and their energies will be related to the matrix elements $\left\langle(j)^{2} J\left|\mathcal{Y}_{\text {eff }}\right|(j)^{2} J\right\rangle$. From Eq. (D.24) it follows that

$$
\begin{aligned}
& \left\langle(j)^{2} J\left|\gamma_{\text {eff }}\right|(j)^{2} J\right\rangle=\left\langle(j)^{2} J|V|(j)^{2} J\right\rangle-\left\langle k_{V}\right\rangle^{2} \sum_{L} \theta_{L} M_{L}^{J} \\
& \left.M_{L}^{J}=(-1)^{J-2 j} \hat{j}^{2} W(j j j j, L, J)<j|| Y_{L}|| j\right\rangle^{2}
\end{aligned}
$$

where $\bar{E}^{-1}\left(\hbar \omega_{L} / C_{I}\right)=-I / C_{L}=-\theta_{L}$ consistent with the assumption discussed above in regard to the transition matrix elements. Examination of the behavior with $L$ and $J$ of the Racah coefficient in Kq. (D.26) shows that the second term in Eq. (D.25) will give a strong attractive contribution to the 
$J=0$ matrix element and will give a repulsive contribution to matrix elements for higher $J$ provided the ${ }_{L}$ fall off sufficiently slowly with increasing $L$. This is the effect required to reproduce the observed spectrum ${ }^{20-25}$ which in turn can be used to fix the $\theta_{L}$ 's.

Note that in computing the renormalization of the bound state matrix elements by this prescription that no contributions from abnormal parity states of the core are included. This is a direct result of the form assumed for the valence core interaction, $\mathrm{Eq}$. (D.6). In the microscopic calculations of Kuo and Brown ${ }^{20-25}$ these contributions are shown to be small and repulsive. Nevertheless, the values of $\theta_{L}$ corresponding to normal parity core excitations determined from the spectrum will be somewhat too small because repulsive terms are neglected. In this work this difficulty is circumvented by determining the $\theta_{L}$ from the decomposition of the $G_{3 p-i n}$ contributions to the $J=0$ matrix elements calculated by Kuo and Brown $20-25$. Deficiencies in their matrix elements should show up as corresponding deficiencies in the results of this work.

This procedure can be extended to more complicated cases. The essential criterion for its applicability is that there are no more ${ }_{L}{ }^{\prime}$ 's to determine than there are matrix elements defined by the spectrum. In a more general case the $\theta_{L}$ 's which are determined may show some configuration dependence which is, of course, expected. The inverse of this process has been used to renormalize bound state matrix elements in the $\mathrm{Pb}^{206}$ calculations by True and Ford. 94 
3. Microscopic Treatment of Core Polarization

In the completely microscopic calculations the quantities to be determined are the proton and neutron transition densities; therefore, interest is in the reduced matrix elements of the operator defined in Eq. (B.21) and Eq. (B. $\left.2 I^{\prime}\right)$.

One Nucleon Outside of a Closed Shell

For a nucleus with one nucleon outside of closed shell the unperturbed valence configurations are the single particle states defined in Section 3 of Appendix $B$. The necessary reduced matrix element corresponding to the first term in Eq. (D.5) is given by Eq. (B.22) and will be called $R_{\tau \tau^{-}}^{L S J}(D)$ where $D$ refers to direct. The reduced matrix element corresponding to second and third term in Eq. (D.5) will be called $\mathrm{R}_{\tau \tau}^{\mathrm{LSJ}}(\mathrm{C})$ where $\mathrm{C}$ refers to core.

In calculating $\mathrm{R}_{\tau \tau}^{\mathrm{LSJ}}(\mathrm{C}), \mathrm{V}^{\text {- }}$ is the "bare" G-matrix or an approximation to it such as the $K-K$ force and $P$ projects onto $2 \mathrm{p}$-Ih states.

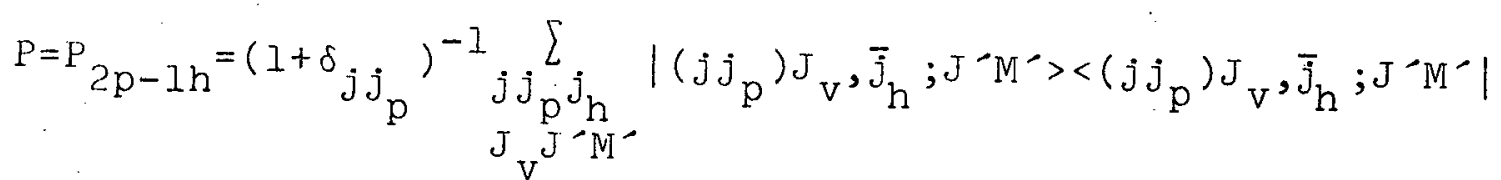

$$
\begin{aligned}
\left|\left(j j_{p}\right) J_{v}, \bar{j}_{h} ; J^{\prime} M^{-}\right\rangle & =\sum_{m_{p}}\left\langle j j_{p} m_{p} \mid J_{v^{M}} M_{v}\right\rangle\left\langle J_{v} j_{h} M_{v}-m_{h} \mid J^{\prime} M^{\prime}\right\rangle \\
& x(-1)^{j_{h}-m_{h}+a_{p}^{+} a_{h}|C\rangle}
\end{aligned}
$$


For convenience reference to the quantum numbers $\tau, \tau_{p}$, and $\tau_{h}$ has been suppressed. In Eq. (D.27) only distinct pairs $\left(j j_{p}\right)$ are included in the sum, i.e. $(1,2)$ is not different from $(2,1)$ and only even values of $J_{i v}$ are allowed when $j=$ $j_{p}$. It is not hard to show that

$$
P_{2 p-1 h}=P_{2 p-1 h}^{1}+P_{2 p-1 h}^{2}
$$

where $\mathrm{P}_{2 \mathrm{p}-\mathrm{ln}}^{\mathrm{i}}$ includes only the terms in Eq. (D.27) with $j=j_{p}$ and

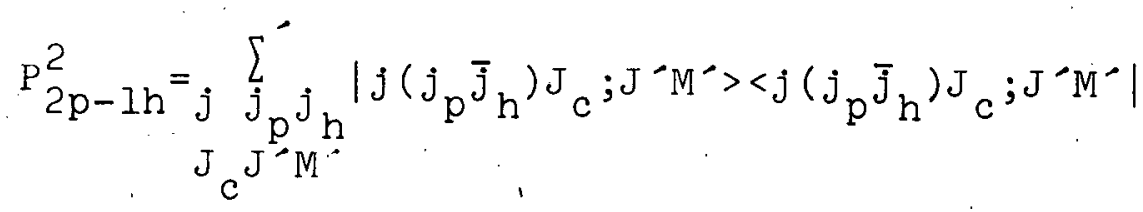

with the prime indicating that terms with $j=j_{p}$ are excluded. The state vectors appearing in Eq. (D.30) are defined in Eq. (B.37). Contributions from $\mathrm{P}_{2 \mathrm{p}-1 \mathrm{~h}}^{\mathrm{l}}$ are excluded in the calculations of this work. In the work reported in Ref. 20-25 and Ref. 88 a quasi-boson assumption is made and intermediate states with a valence nucleon and like core nucleon in the same orbital are allowed. This assumption amounts to including terms with $j=j_{p}$ in Eq. (D.30) instead of the manner prescribed by $\mathrm{P}_{2 \mathrm{p}-\mathrm{Ih}}$. In this case there are only two extra-core nucleons in the intermediate states and it is not difficult to carry out the complete calculation while maintaining consistency with the Pauli principle; however, this is not true when the intermediate states have 
inore than two extra-core nucleons. In any event the neglected contributions are likely to give only a small percentage of the total effect.

In order to illustrate the derivation of the formulas used in the microscopic calculations note that the third term in Eq. (D.5) takes the form

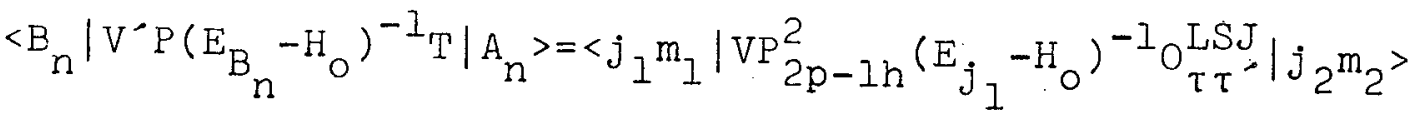

$$
\begin{aligned}
& =-\sum_{j}^{j} E(p h)^{-1}<j_{I}|V| j_{2}\left(j_{p} \bar{j}_{h}\right) J ; j_{I}><j_{2}\left(j_{p} \bar{j}_{h}\right) J ; j m_{I}\left|O O_{\tau \tau}^{L S J}\right| j_{2} m_{2}> \\
& \tau p{ }^{\tau} h
\end{aligned}
$$

where $E(p h)=E_{p}-E_{h}+E_{j 2} \sim E_{p}-E_{h}$ and $\tau_{p}$ and $\tau_{h}$ have been introtroduced explicitly. In writing Eq. (D.31) use is made r.f Eq. (B.38) which shows that $j$ must equal $j_{2}$ or $j_{p}$ and $J_{c}$ must equal $\mathrm{J}$. Since the sum of $j j_{\mathrm{p}}$ in $\mathrm{Eq} \cdot(\mathrm{D} .30$ ) includes distinct pairs and $j \neq j_{p}$ one is free to choose $j=j_{2}$ and sum over $j_{p} \neq j_{2}$. Further, the matrix element of $V$ vanishes unless $j_{1} m_{1}=J^{\prime} M^{\prime}$. In the occupation number representation $\mathrm{V}$ is written

$$
V=\frac{1}{2} \sum_{\alpha \beta \gamma \delta}<\alpha \beta|v| \gamma \delta>\mathrm{a}_{\beta}^{+} \mathrm{a}_{\alpha}^{+} \mathrm{a}_{\gamma} \mathrm{a}_{\delta}
$$

where $\langle\alpha \beta|V| \gamma \delta\rangle$ is an unsymmetrized two-body matrix element. Using Eq. (D.32) it can be shown that bound state matrix element in Eq. (D.3I) is given by 


$$
\begin{aligned}
& 272
\end{aligned}
$$

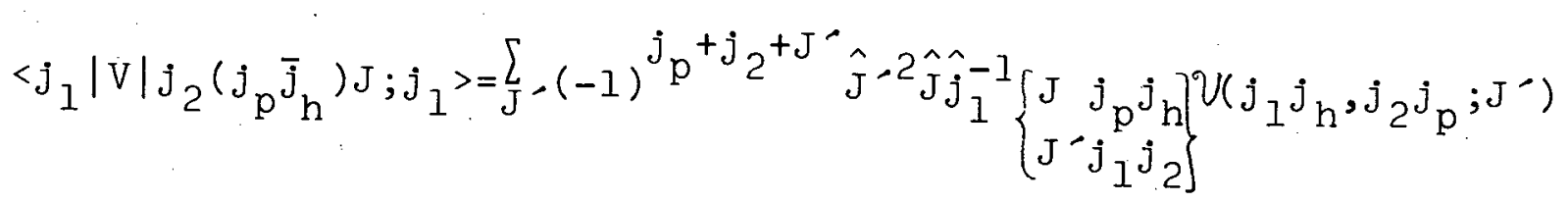

$$
\begin{aligned}
& \text { (D.33) } \\
& \text { where }\left(j_{1} j_{h}, j_{2} j_{p} ; J^{\prime}\right)=\left[\left(I+\delta_{j_{1} j_{h}}\right)\left(I+\delta_{j_{2} j_{p}}\right)\right]^{l / 2} \\
& x V\left(j_{1} j_{h}, j_{2} j_{p} ; J^{\prime} T=I\right) \\
& \left(\tau_{1}=\tau_{h} ; \tau_{2}=\tau_{p}\right) \\
& x \frac{1}{2}\left\{V\left(j_{1} j_{h}, j_{2} j_{p} ; J^{-} T=0\right)+V\left(j_{I} j_{h}, j_{2} j_{p} ; J^{\prime} T=I\right)\right\} \quad\left(\tau_{I} \neq \tau_{h} ; \tau_{2} \neq \tau_{p}\right)
\end{aligned}
$$

with $V\left(j_{1} j_{h}, j_{2} j_{p} ; J^{\prime} T\right)$ designating a two-body matrix element between antisymmetrized two-particle states coupled to total angular momentum $J^{\prime}$ and iso-spin T. In deriving this result using Eq. (D.32) contractions leading to one-body potential terms in Eq. (D.33) are neglected. For the $\left(p, p^{\prime}\right)$ reaction and electromagnetic transition $\tau_{1}=\tau_{2}$ and $\tau_{p}=\tau_{h}$. When the valence nucleon and excited core nucleon are of the same type only the $T=1$ part of the particle-core interaction is effective, whereas both the $\mathrm{T}=0$ and $\mathrm{T}=1$ parts of this interaction are effective when these nucleons are different. The product of the matrix element (D.33) and $-E(\mathrm{ph})^{-1}$ is the probability amplitude for the (ph) component in the final state wave function.

Combining Eq. (D.33) and Eq. (B.38) gives the following expression for the reduced matrix element corresponding to Eq. (D.31)

$$
\begin{aligned}
& R_{\tau \tau}^{L S J}\left(C_{2}\right)=-\sum_{j}^{L} j_{h} \delta_{\tau \tau^{\prime}, \tau_{h} \tau_{p}} E(p h)^{-1}(-I)^{j} 2^{+j} p^{+J} \hat{J}^{-2} \hat{j}_{p} \hat{j}_{I}^{-1} \\
& T_{\mathrm{J}}^{\mathrm{T}} \mathrm{T} h
\end{aligned}
$$




$$
x\left\{\begin{array}{ll}
J & j_{p} j_{h} \\
J^{\prime} j_{1} j_{2}
\end{array}\right\} V\left(j_{1} j_{h}, j_{2} j_{p} ; J^{\prime}\right)<j_{p}|| o^{L S J}|| j_{h}>.
$$

A similar expression can be obtained for the reduced matrix element corresponding to the second term in Eq. (D.5). This is called $R_{\tau \tau}^{L S J}\left(C_{1}\right)$ and differs from Eq. (D. 35) by a phase and the interchange of $j_{1}$ and $j_{2}$. The sum of the two contributions from core polarization to the transition density is

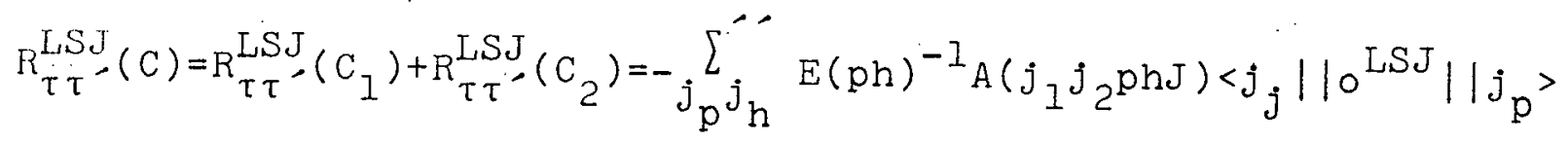

$$
\begin{aligned}
& \tau p \tau_{h} \\
& A\left(j_{1} j_{2} p h J\right)=\sum_{J}-\delta \tau^{\prime}, \tau_{h} \tau_{p}(-1)^{J-j_{p}+j_{I}} \hat{J}^{2} \hat{j}_{h^{\prime}} \hat{j}_{1}^{-1} \\
& \left.x\left[\left\{\begin{array}{l}
j_{p} j_{h} J^{\prime} \\
j_{2} j_{I} J^{\prime}
\end{array}\right\}\right\}\left(j_{p} j_{1}, j_{h^{j}} j_{2} ; J^{\prime}\right)+(-1)^{S+J}\left\{\begin{array}{l}
j_{p} j_{h} J \\
j_{I} j_{2} J^{\prime}
\end{array}\right\} V\left(j_{p} j_{2}, j_{h^{\prime}} ; J^{\prime}\right)\right]
\end{aligned}
$$

where the double prime on the sum over $j_{p} j_{h}$ indicates that the first term in [] is not included when $j_{p} \tau_{p}=j_{I} \tau_{I}$ and the second term in [] is omitted when $j_{p} \tau_{p}=j_{2} \tau_{2}$.

It was pointed out previously that there is no breaking of the valence transition selection rules when the macroscopic treatment of core polarization is used. Consider the transition where the valence nucleon goes from an. $s_{1 / 2}$ orbit to the $d_{5 / 2}$ orbit. Without core polarization this transition can only go with L-transfer equal to 2. From Eq. (D.36) it can be seen that $L=4$ is also allowed, $i . e$. assume $|C\rangle$ contains a filled $p$-shell and consider a $f_{7 / 2}-p_{3 / 2}{ }^{-1}$ particle- 
hole pair which gives a contribution for $L=4$. This point is probably academic as the $L=4$ contribution to the transition. is not likely to be as important as that from $L=2$, but it does indicate that core polarization can effect the valence transition selection rules.

Two Nucleons Outside of a Closed Shell

For two nucleons outside of a closed shell the only transitions considered are between states where the valence configurations are the allowed couplings of two like nucleons in the same orbit. The wave functions for these configurations were defined in Eq. (B.35) and Eq. (B.36) gives $R_{\tau \tau}^{\mathrm{LSJ}},(\mathrm{D})$. For this case

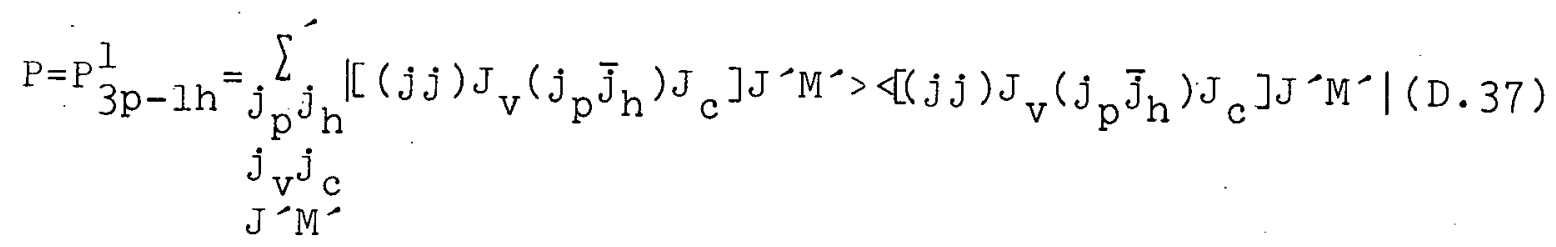

where reference to $\tau_{,} \tau_{p}$, and $\tau_{h}$ is again suppressed and the sum excludes terms with $j=j_{p}$ and odd values of $J_{v}$. The state vectors appearing in the projection operator were defined in Eq. (B.39). Using the notation of the last section

$$
\begin{aligned}
R_{\tau \tau}^{L S J}-\left(C_{2}\right)= & -\sum_{j_{p} j_{h}}^{\prime} E(p h)^{-1}<(j)^{2} J_{B}|V|\left[(j j) J_{A}\left(j_{p} \bar{j}_{h}\right) J\right] J_{B}> \\
& \tau_{p} \tau_{h} \\
& x<\left[(j j) J_{A}\left(j_{p} \bar{j}_{h}\right) J\right] J_{B}|| O_{\tau \tau}^{L S J}||(j)^{2} J_{A}>
\end{aligned}
$$

where Eq. (B.40) has been used along! with the properties of $\mathrm{V}$ to eliminate some of the summation. 
'I'he matrix element of $\mathrm{V}$ appearing in Eq. (D.38) is

$$
\begin{aligned}
& \left.<(j)^{2} J_{B}|V|\left[(j j) J_{A}\left(j_{p} \bar{j}_{h}\right) J\right] J_{B}\right\rangle=
\end{aligned}
$$

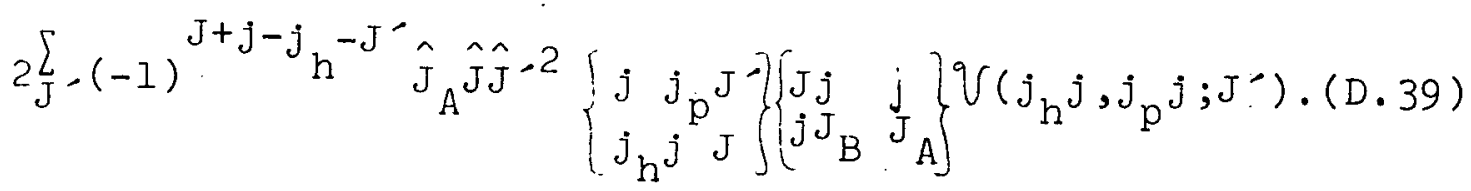

Multiplying result (D.39) by $-E(p h)^{-1}$ gives the probability amplitude for the ( $p h$ ) component in the final state wave function. Inserting the result of Eq. (B.40) and Eq. (D.39) into Eq. (D.38) leads to

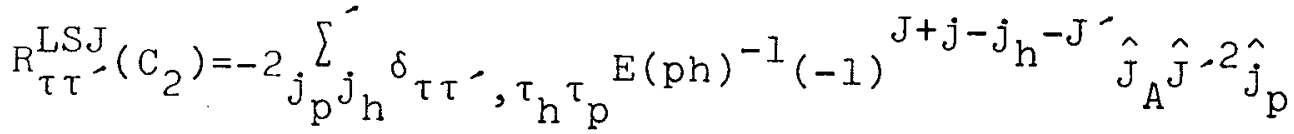

$$
\begin{aligned}
& \tau_{\mathrm{J}}^{\tau}{ }^{\tau} \mathrm{h}
\end{aligned}
$$

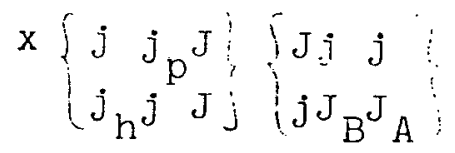

$$
\begin{aligned}
& x:\left(j_{h}{ }^{j, j} j_{p} j ; J^{-}\right)<j_{p} \| o^{L S J}|| j_{h}>\text {. }
\end{aligned}
$$

Combining this result with the corresponding result for $\mathrm{R}_{\tau \tau}^{\mathrm{LSJ}}\left(\mathrm{C}_{I}\right)$ gives the following result.

$$
R_{\tau \tau^{\prime}-(C)=-}^{L_{j}} \sum_{j_{p} j_{h}}^{\prime} E(p h)^{-I} A(j p h J)<j_{h}\left\|0^{L S J}\right\| j_{p}>
$$

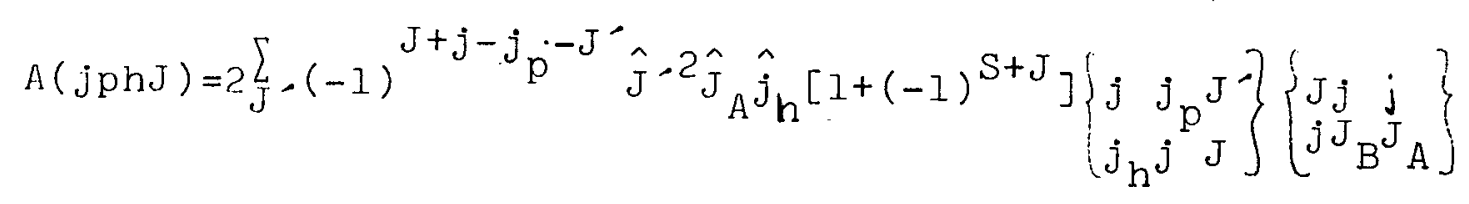

$$
x ! f\left(j_{h} j, j_{p} j ; J^{\prime}\right)
$$


As in the valence transition $L$ must be even and when $J=L$ and $S=I A(j p h J)$ vanishes; therefore, spin-flip is still forbidden for a transition starting from the state $\mathrm{J}_{\mathrm{A}}=0$. The inclusion of core polarization does not lead to any breaking of the valence transition selection rules in these transitions. When $J_{A}=0, L=J=J_{B}$, and $S=0, A(j p h J)$ becomes $A(j p h J)=\frac{4 \hat{j}_{h}}{j \hat{\lambda}} \sum_{J}-(-1)^{j} p^{+j+J \hat{J}^{-2}}\left\{\begin{array}{c}j_{p} j_{h} J \\ j j J\end{array}\right\} V\left(j_{p} j ; j_{h} j ; J^{\prime}\right) \quad$ (D. 42) Phase of Microscopic Core Polarization Contributions The formula obtained for treating core polarization using the macroscopic vibrational model to describe the core clearly displayed the relative phase of the core and direct contributions to transitions. The phase of $R_{\tau \tau}^{L S J}$ (C) defined in Eq. (D.36) and Eq. (D.4I) with respect to the corresponding $R_{\tau \tau}^{L S J}(D)$ is not apparent from inspection. It is useful to examine this phase relation.

To do this it is necessary to express the two-body matrix element in terms of multipoles of the two body force.

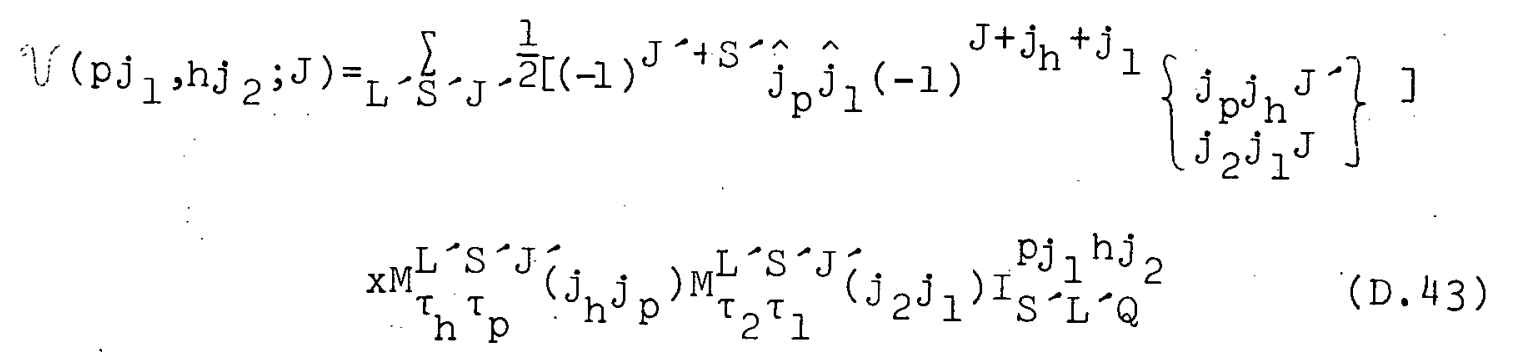




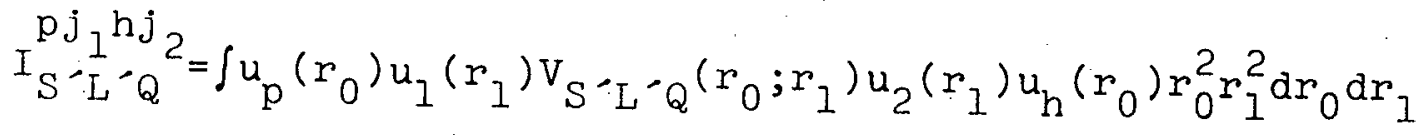

$$
\begin{aligned}
& +\int u_{p}\left(r_{0}\right) u_{I}\left(r_{I}\right) v_{S^{\prime} L^{\prime} Q^{2}}^{E}\left(r_{0} ; r_{I}\right) u_{2}\left(r_{0}\right) u_{h}\left(r_{I}\right) r_{0}^{2} r_{I}^{2} d r_{0} d r_{1}
\end{aligned}
$$

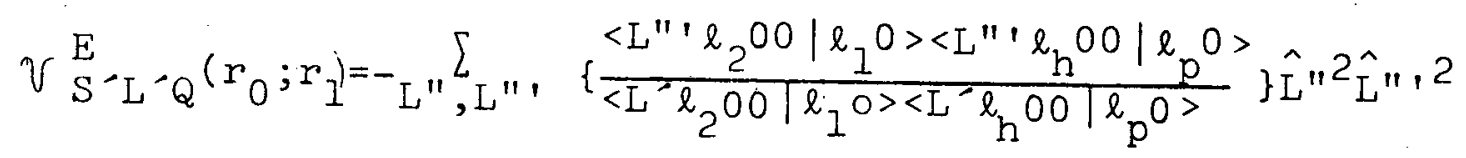

$$
\begin{aligned}
& x W\left(\ell_{2} \ell_{p} \ell_{I} \ell_{h} ; L^{\prime \prime} L^{\prime}\right) W\left(l_{2} \ell_{p} \ell_{I} \ell_{h} ; L^{\prime \prime} L^{\prime \prime}\right) V_{S}^{E} L^{\prime \prime Q}\left(r_{0} ; r_{I}\right)
\end{aligned}
$$

This expression for the two-body matrix element is obtained by following the procedure used in chapter 2 for decomposing the D.W.A. transition amplitude $Q$ designates the $p-p(n-n)$ or $p-n$ force as $\tau_{h} \tau_{p}$ is the same or opposite to $\tau_{2} \tau_{1}$ $\mathrm{V}_{\mathrm{S}^{\prime} \mathrm{L}^{\prime} \mathrm{Q}^{\mathrm{Q}}}\left(\mathrm{r}_{0} ; \mathrm{r}_{1}\right)$ is the exchange interaction defined by Eq. (2.23),

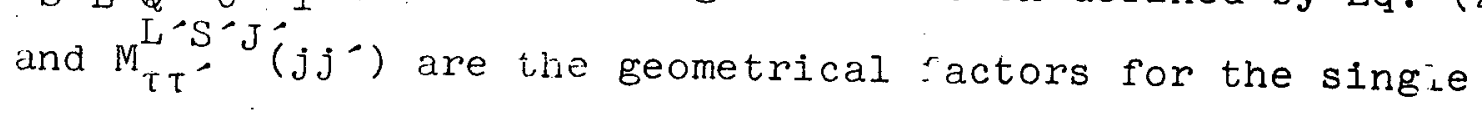
particle transition density defined according to Eq. (B.6) and Eq. (B. $\left.{ }^{\circ}\right)$. The second integral in Eq. (D.44) is the exchange integral which is expected to be in phase with the direct integral for a short range even state force since $V_{S^{\prime} L^{\prime} Q^{-}}^{E}\left(r_{0} ; r\right)=V_{S^{\prime} L^{\prime} Q^{\prime}}\left(r_{0} ; r_{I}\right)$ for a zero range even state force. Using $\mathrm{Eq}$. (D. 43) in the definition

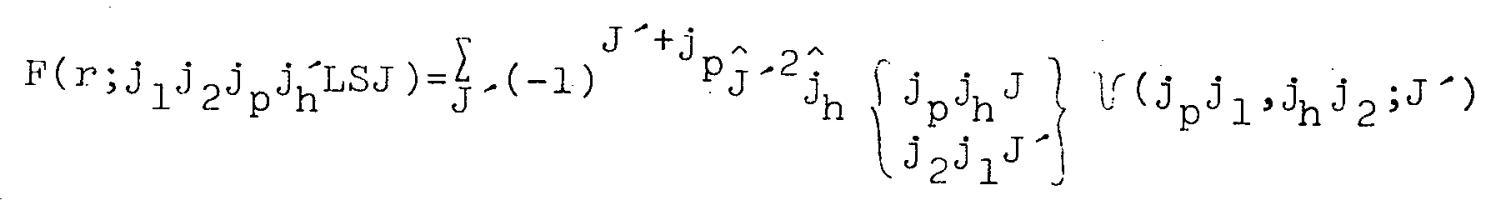

$$
\begin{aligned}
& x<j_{h}|| o^{L S J}|| j_{p}>
\end{aligned}
$$

leads to 


$$
\begin{aligned}
& 278 \\
& F\left(r ; j_{1} j_{2} j_{p} j_{h}^{\prime L S J}\right)=(-1)^{j_{I}+1} \hat{j}_{I} M_{\tau_{2} \tau_{1}}^{L S J}\left(j_{2} j_{I}\right) u_{p}(r) u_{h}(r) \\
& x_{L} \sum_{S}-(-1)^{S+S_{D}^{\prime}\left(j_{1} j_{2} j_{p} j_{h}, J ; L^{\prime} S^{\prime}, L S ; Q\right)}
\end{aligned}
$$

and

$$
\begin{aligned}
F\left(r ; j_{2} j_{1} j_{p} j_{h}^{L L S J}\right)= & (-1)^{J}(-1)^{j_{1}+1}{ }_{M_{\tau_{2}}^{L} \tau_{1}}\left(j_{2} j_{1}\right) u_{p}(r) u_{h}(r) \\
& x_{L^{L}} S^{-(-1)} S_{D}\left(j_{1} j_{2} j_{p} j_{h}, J^{\prime} ; L^{\prime} S^{\prime}, L S ; Q\right)
\end{aligned}
$$

where

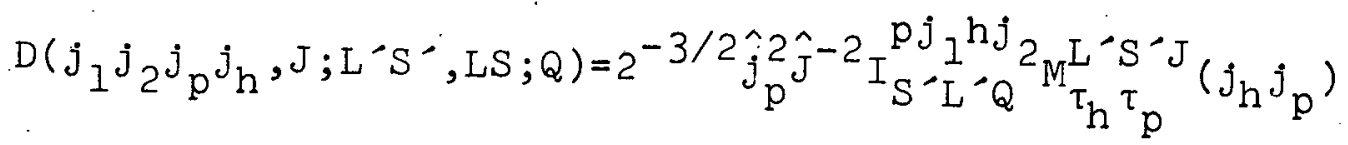

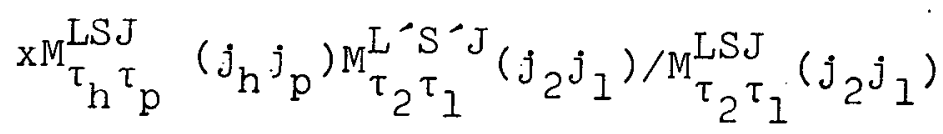

With these relations $\mathrm{Eq}$. (D.36) can be written

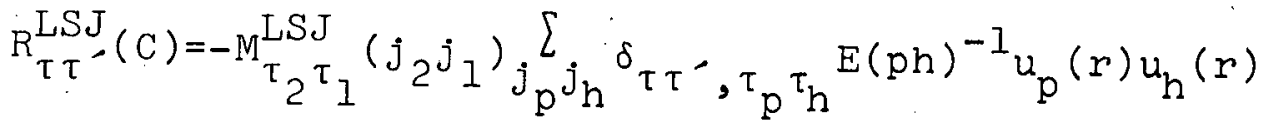

$$
\begin{aligned}
& \tau^{\tau} \tau_{h} \\
& x_{L^{\prime}} S_{S}-\left[(-1)^{S+S^{-}}+I\right] D\left(j_{1} j_{2} j_{p} j_{h}, J ; L^{-} S^{-}, L S ; Q\right) .
\end{aligned}
$$

The sum on $S^{\prime}$ can be removed as only the term $S^{\prime}=S$ gives a nonvanishing contribution. This gives

$$
\begin{aligned}
& R_{\tau \tau^{\prime}}^{L S J}(C)=-M_{\tau_{2} \tau_{I}}^{L S J}\left(j_{2} j_{I}\right) 2 \sum_{j_{p} j_{h}} \delta \tau_{\tau \tau^{\prime}, \tau_{p} \tau_{h}} E(p h)^{-1} u_{p}(r) u_{h}(r) \\
& \tau p \tau_{h} \\
& x_{L} \sum_{-D}\left(j_{1} j_{2} j_{p} j_{h}, J ; L^{-} S, L S ; Q\right) \text {. }
\end{aligned}
$$


A similar result is obtained for the $R_{\tau \tau}^{L S J}$ (C) obtained by using Eq. (D.42) in Eq. (D.41). This is

$$
\begin{aligned}
& R_{\tau \tau}^{J O J}(C)=-\frac{2}{\lambda} M_{\tau}^{J O J}(j j) 2 \sum_{\substack{j \\
\tau_{p} j_{h}}}^{\sum_{j} \delta \tau, \tau p} \tau E(p h)^{-1} u_{p}(r) u_{h}(r) \\
& x D\left(j j j_{p} j_{h}, J ; J O, J O ; Q\right) .
\end{aligned}
$$

There is no sum on $L^{\prime}$ in Eq. (D.52) as the transition being considered here is of normal parity and has only one allowed value of $\mathrm{J}$. Similarly in Eq. (D.5I) only $\mathrm{L}^{-}=\mathrm{J}$ contributes to the $\operatorname{triad}(\mathrm{LSJ})=(\mathrm{JOJ})$.

Eq. (D.5I) and Eq. (D.52) have the form needed to see the effect of core polarization, as treated in this microscopic picture, on transitions. In both equations the negative of the geometrical factor for the valence transition appears as an overall multiplicative factor. This does not mean that violations of the valence transition selection rules are not possible since this geometrical factor also appears in the denominator of $D$. Only triads allowed in the valence transitions will be considered here.

To see the phase it is only necessary to consider particle-hole pairs whose radial wave functions are similar to those of the active valence nucleon. The largest values of $\mathrm{I}_{\mathrm{S}-\mathrm{L}{ }^{-} \mathrm{h}}^{\mathrm{hj}}$ will occur in these instances and this radial integral will have the sign of the $S^{\prime} Q$ component of the two body force. Inspection of Eq. (D.49) shows that 
$D\left(j_{1} j_{2} j_{p} j_{h}, J ; L S, L S ; Q\right)$ has the same sign as $I_{S L Q}^{p j}{ }_{1}^{h j_{2}}$; therefore, for the triads (JOJ) and (JIJ) the direct and core polarization contributions will be in phase if the corresponding component of the two-body force is attractive. Only the "spin-flip" component of the $p-p(n-n)$ force used in this work is repulsive. Because of this when the valence nucleons are protons (neutrons), proton-proton hole (neutron-neutron hole) excitations will decrease the ( $J I J)$ transition amplitude. The same arguments hold for the triads $(J \pm l, l, J)$ although there is an additional complication because the phase depends on the sum of two terms. When $D\left(j_{1} j_{2} j_{p} j_{h} J ; L S, L S ; Q\right)$ is dominant the conclusions above will hold. This is likely to be true for the $(J-l, l, J)$ triad as $D(L S, L S)$ will by proportional to the $L=J-1$ multipole coefficient of the twobody force while $D(L S, L S)$ will be proportional to the $L=J+1$ multipole coeffecient. For the triad $(\mathrm{J}+\mathrm{I}, \mathrm{I}, \mathrm{J})$ this situation is reversed.

4. Microscopic Empirical Formula

Here a formula for computing, from the effective charges, the enhancement of a cross section in the $\left(p, p^{\prime}\right)$ reaction due to core polarization, is derived on the basis of microscopic considerations alone. The argument is orginally due to Atkinson and Madsen ${ }^{19}$ and is given here in the notation of this paper.

For a normal parity transition with some degree of collectivity the triad (LOL) gives the dominant contribution 
to the cross section. The form factor designated by this triad is

$\tilde{F}^{L O L}(r)=\int_{0}^{\infty}\left\{\gamma_{O p L}\left(r ; r^{\prime}\right) F_{p}^{L O L}\left(r^{\prime}\right)+\gamma_{O n L}^{\prime}\left(r ; r^{\prime}\right) F_{n}^{L O L}\left(r^{\prime}\right)\right\} r^{-2} d r^{\prime}$

as specified in Eq. (2.59"). $F_{p}^{L O L}$ and $F_{n}^{L O L}$ are the proton and neutron transition densities, respectively, and $\gamma_{O p L}$ and " $V_{n L}$ are the multipole coefficients of the non-"spinflip" components of the $p-p$ and $p-n$ forces with the exchange interaction included. Correspondingly for $\gamma$-decay Eq.(C.17) gives

$$
B(E L)=\frac{2 J_{B}+1}{2 J_{A}+I} \frac{I}{2}\left|\int_{0}^{\infty} r^{L+2} F_{p}^{L \odot L}(r) d r\right|^{2} e^{2} .
$$

The neutron and proton transition densities have two components,

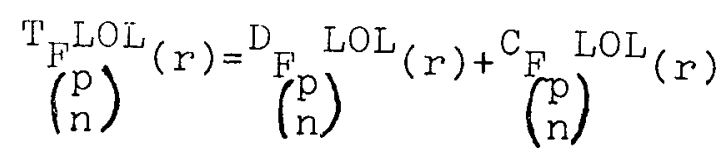

where $D$ is the direct or valence component and $C$ is the core component.

Two assumptions make it possible to relate, algebraically, the effective charges of the valence nucleons to analagous enhancement factors for the ( $\left.p, p^{\prime}\right)$ reaction. One is to neglect radial differences between the proton and neutron transition densities and their direct and core components. The second is to assume that different components of the projectile-target interaction have the same 
radial form or, that "equivalent" components with the same radial form can be defined. The $I F$ range "equivalent" impulse approximation pseudo-potential given in Chapter 3 can be used in this context. The local approximation to the exchange component of the D.W.A. transition amplitude is an implicit uncertainty in the second assumption.

The total proton transition density can be written

$$
F_{p}(T)=\frac{1}{2}\left\{F_{p}(T)+F_{n}(T)\right\}+\frac{1}{2}\left\{F_{p}(T)-F_{n}(T)\right\}
$$

where $F_{n}(T)$ has been introduced so that $F_{p}(T)$ is expressed in terms of iso-scalar and iso-vector components. An isoscalar transition is defined by the condition $F_{p}(T)=F_{n}(T)$. In terms of the iso-scalar and iso-vector effective charges,

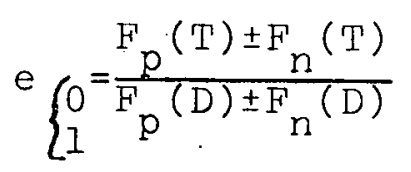

Eq. (D. 56) becomes

$$
\begin{aligned}
F_{p}(T) & =\frac{1}{2} e_{0}\left\{F_{p}(D)+F_{n}(D)\right\}+\frac{1}{2} e_{I}\left\{F_{p}(D)-F_{n}(D)\right\} \\
& =e_{p} F_{p}(D)+e_{n} F_{n}(D)
\end{aligned}
$$

where the proton and neutron effective charges are

$$
e_{p}^{p}=\frac{1}{2}\left(e_{0} \pm e_{1}\right)
$$

Correspondingly, 